DATA LIBRARY

Woods Hoie Ocsanographic Inslitutios

Barataria Bay model, A report to the Freeport Sulpher Company by William Von Arx

$$
1950
$$

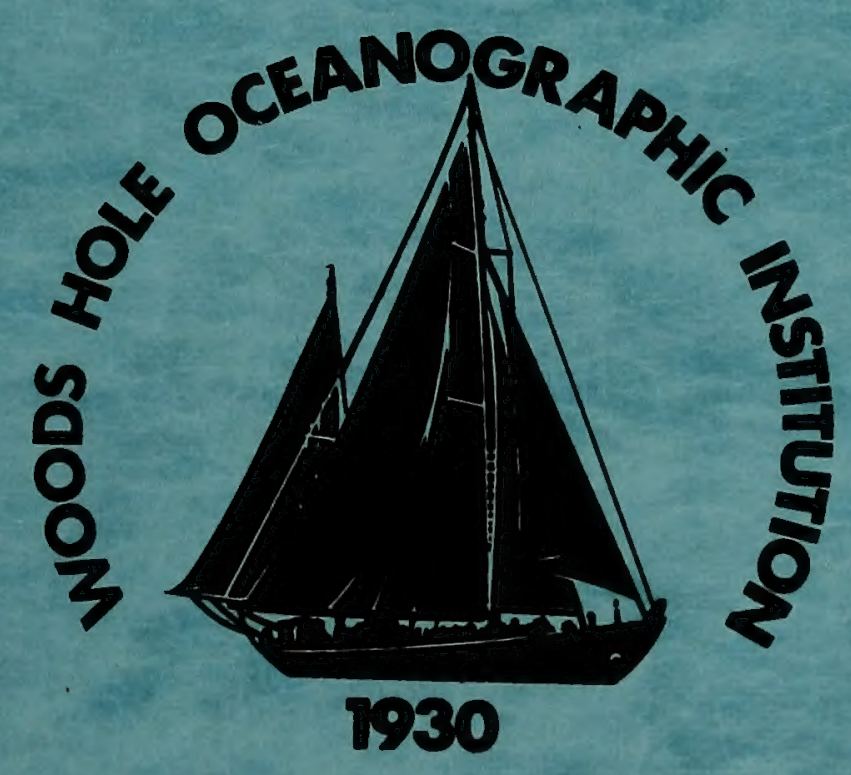







\title{
THE BARATARIA BAY MODET
}

\author{
A Report to \\ the
}

FREEPORT SULPHUR COMPANY

by

William S, von Arx

** *

Woods Hole, Massachusetts

August 1950

COPY NO. 7

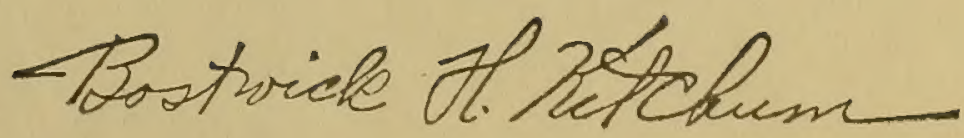




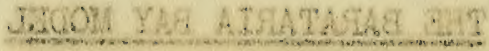

0 करणदु के

aids

Jhe

\author{
षd \\ Xur mov + 2 ms DLFim \\ $\therefore 8 \div$

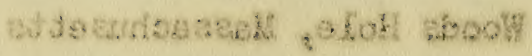 \\ Qeed inerams
}

6.012 .1900 


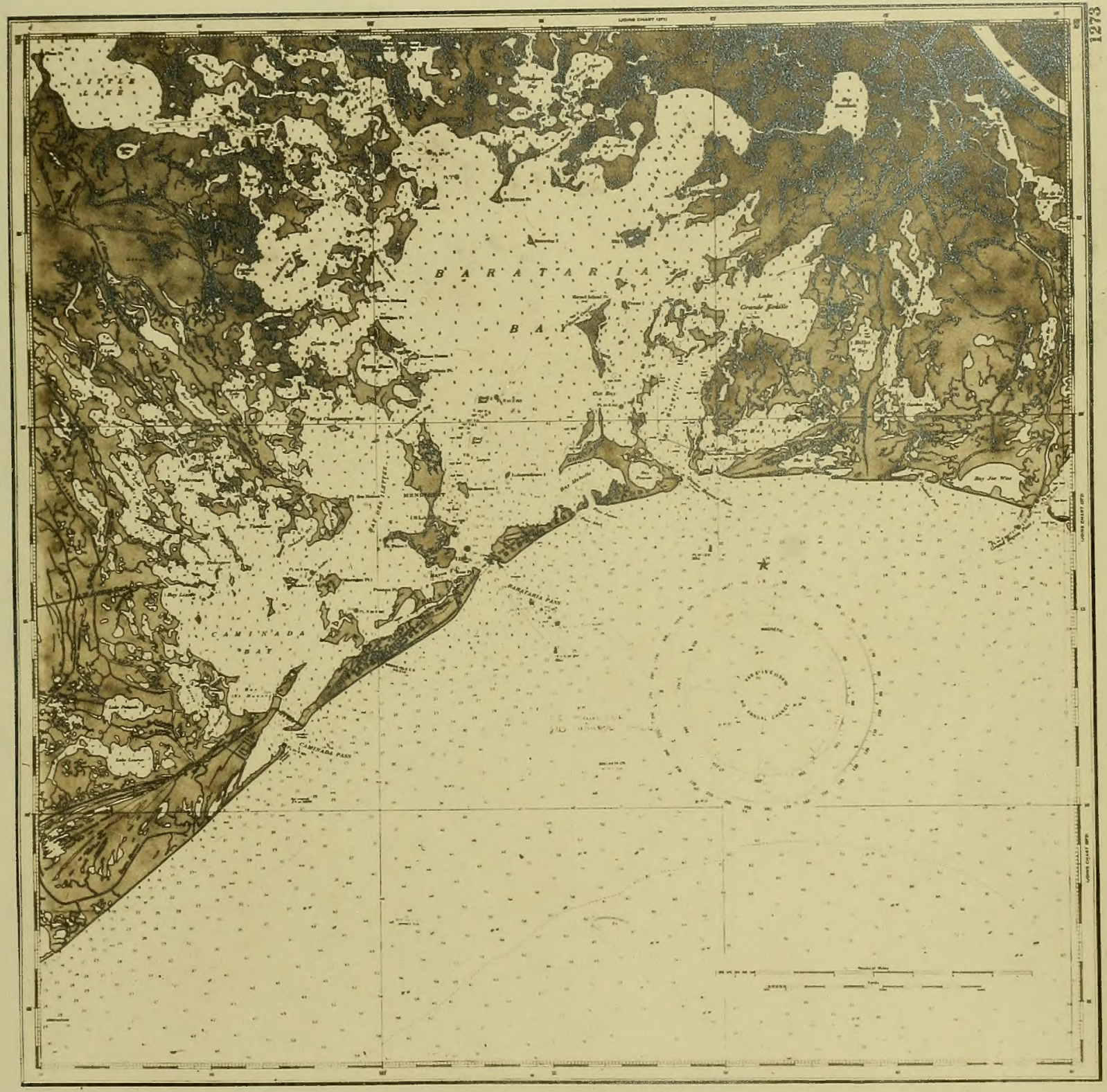

Frontispiece

The area represented by the Barataria Bay model. 



\section{Contents}

\section{Page}

I. Summary - Introduction I

II. The Role of Modern Hydraulic Models 9

III. The Scales of the Barataria Bay Model 11

IV. Reconciliation of Kinematic and Dynamic Processes 16

V. The Tide Machine 24

VI. Kinematic Verification of the Kodel 29

VII. Specifications of the Model Tests 32

VIII. Chemistry of the Bleed Water Tracer 34

IX. Awxiliary Pumping Systems 39

X. The Reliability of the Model Tests 43

XI. "First Arrival" Tests 44

Appendix I Schedule of procedure in model tests

II Photographic and Chemical log

III Drawings of results of model tests

IV Tide records attached to Copy No. 1. The tides are also indicated on the time-lapse motion and still pictures of the model tests

v U. S. Weather Bureau reports from Grand Is Ie, Louisiana station attached to Copy No. I 



\section{SUMMAARY - INTRODUCTION}

In late December 1948, the Freeport Sulphur Company requested the writer to undertake construction of an hydraulic model of the Barataria Bay area in which their Lake Grande Ecaille, Louisiana, mine is located (see frontispiece adapted from Chart 1273 U.S.C.\& G.S.). In this area, roughly 25 miles square, the characteristic depth of water is about three feet, necessitating either a very large model or a highly distorted smaller model. It was decided to make the model eight feet square, and employ a horizontal scale of $1 / 20,000$ and a vertical scale of $1 / 192$; that is, employ a vertical exaggeration of $1 / 104$. The model was built and operated on the property of the Woods Hole Oceanographic Institution in Woods Hole, Massachusetts.

The model was constructed on a strong bed of $2 \times 12$-inch fir timbers (fig. I) arranged to form a tight network of triangular voids. The framework was then covered with $3 / 4$ inch marine plywood in steps (fig. 2) so cut that the treads had the shape of the intervals between successive 12-foot contours on the bottom topography in the prototype (fig. 3). This "staircase" was boxed in by a heavy combing, caulked, (fig. 4) and covered with a lath of hardware cloth (fig. 5). Over this a 4-inch thickness (3/4 ton) of Hydrocal B-Il (precision pattern-maker's $\mathrm{CaSO}_{4}$ plaster) was cut as a monolith in which the intricate modeling of the bays and islands could be cut with rotary tools. The layout of the land and islands 

was projected from the top of a tower (fig. 6) at a height of 16 feet above the model surface, traced off, (fig. 7), and routed in relief ( $f i g .8$ ) so that sea level lay $1 / 4$ inch below the land surface. The soundings were then entered on the sea level surface from the same lantern slide and projector, and additional soundings were entered from the original data sheets provided by the U. S. Coast \& Geodetic Survey. Soundings were realized by routing between carefully drawn contours (fig. 9). The steps were then blended with hand tools (fig. 10). The resulting surface (fig. 11) is probably accurate to \pm 0.015 inch ( \pm 0.25 "foot") vertically and \pm 0.03 inch ( \pm 50 "feet") horizontally. Sea level was established as a level plane $( \pm .005$-inch) by means of repeated checks with a precise transit on the pair of rails from which the routing tools were suspended.

Gravitational (Froude) scaling of the kinematic ratios of time, velocity and discharge was used unmodified except at the passes connecting the bays with the Gulf of Mexico. In each of the passes the velocity and the turbulence (measured in terms of eddy diffusion) of flow were initially too high. In order to correct this common situation, roughness was artificially increased in the passes by means of wire screens ( $20 \times 20$ mesh). Increased roughness in these critical channels not only decreased the velocities so as to agree with the Froude scales but corrected to an acceptable degree the characteristics of channel turbulence as well. To verify the over-all kinematic behavior of 



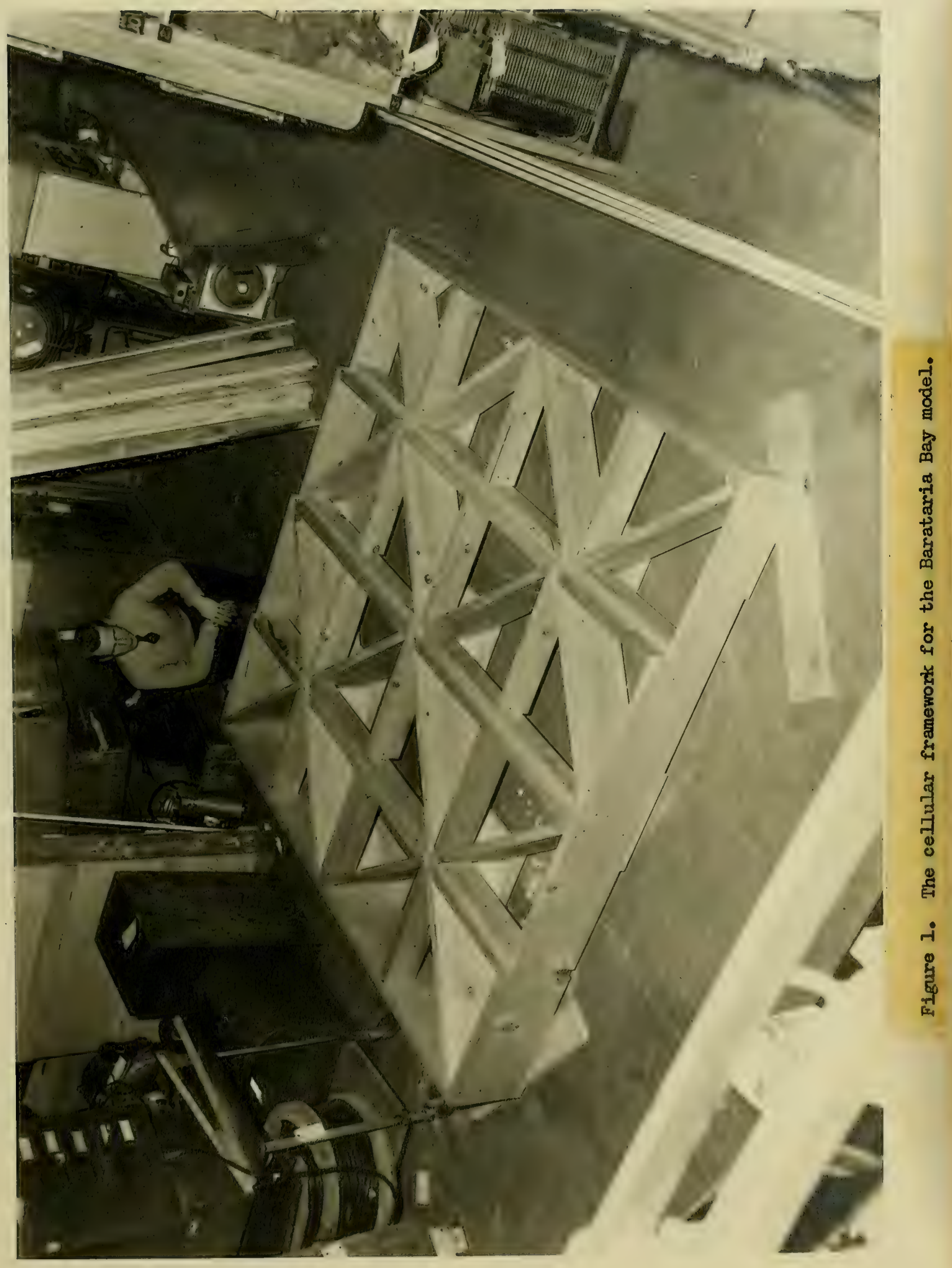





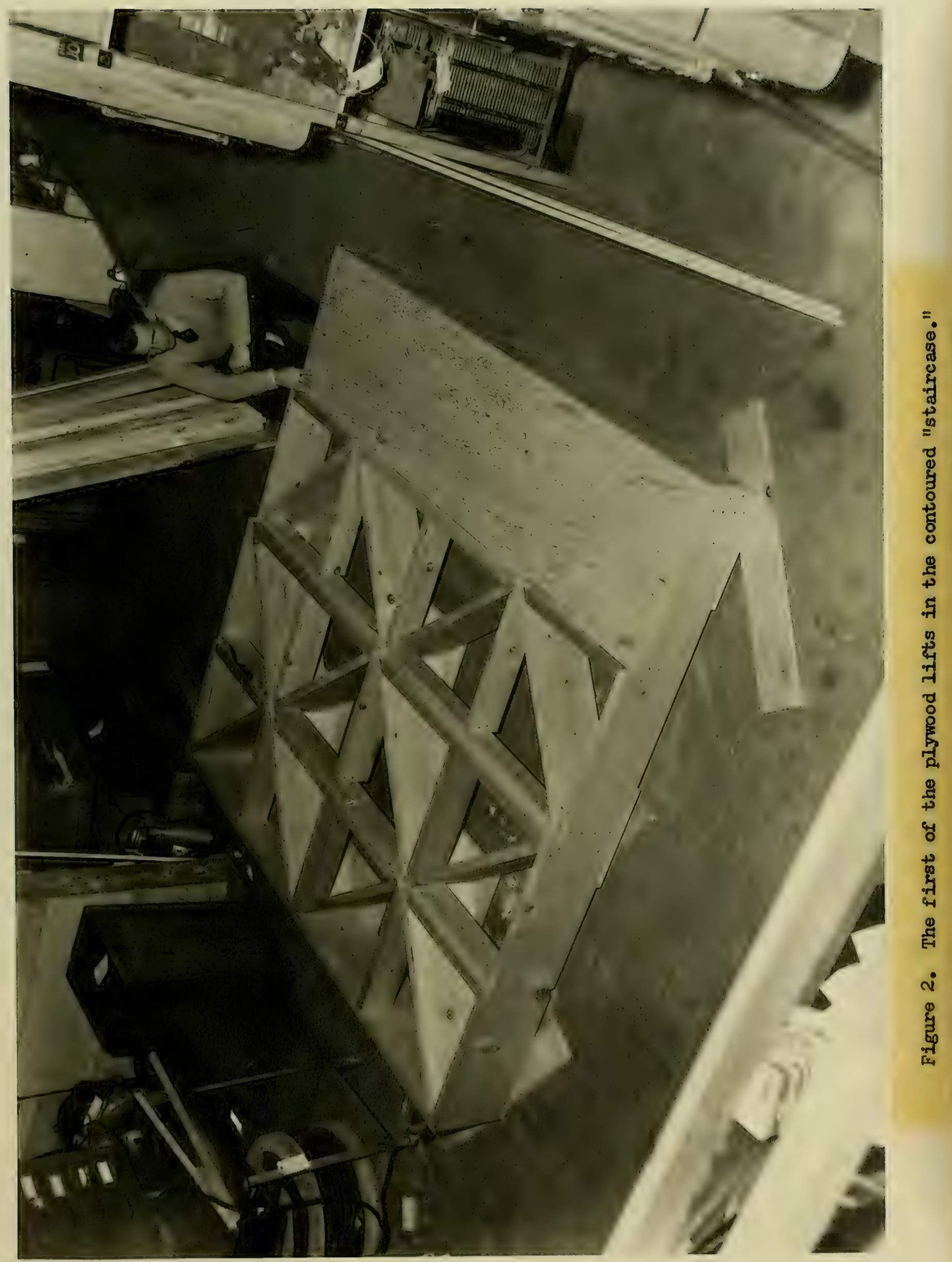





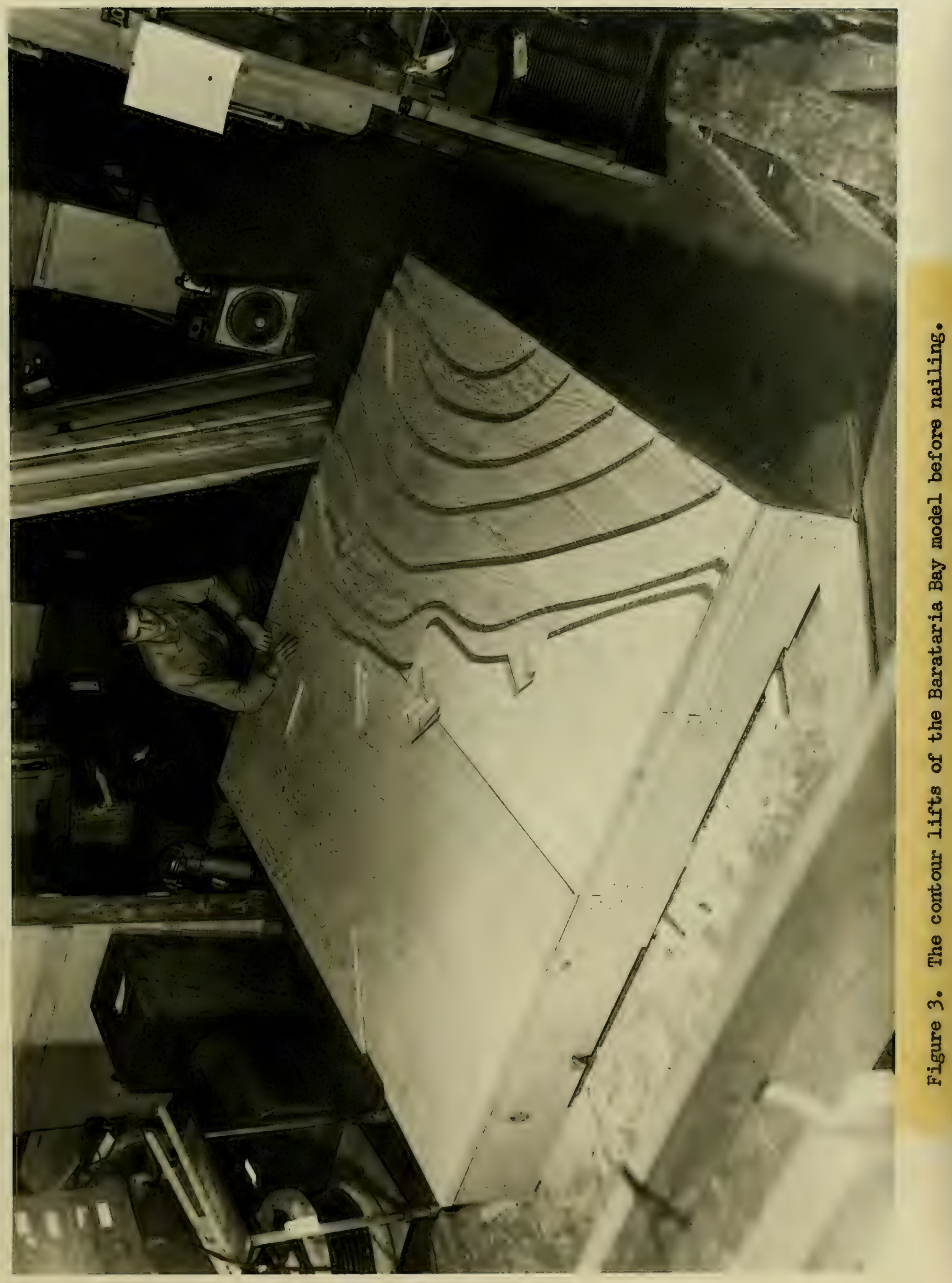





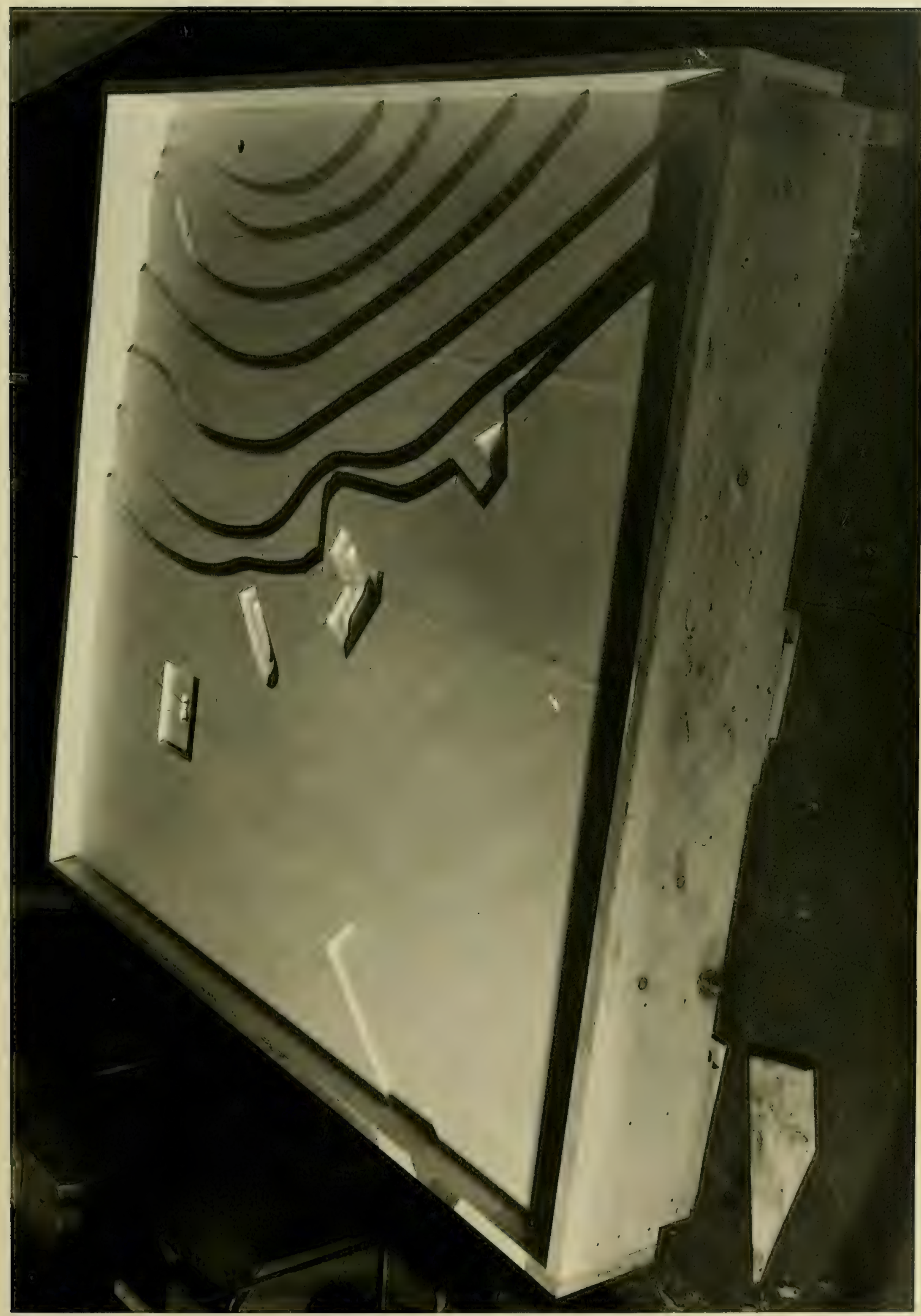

엉 



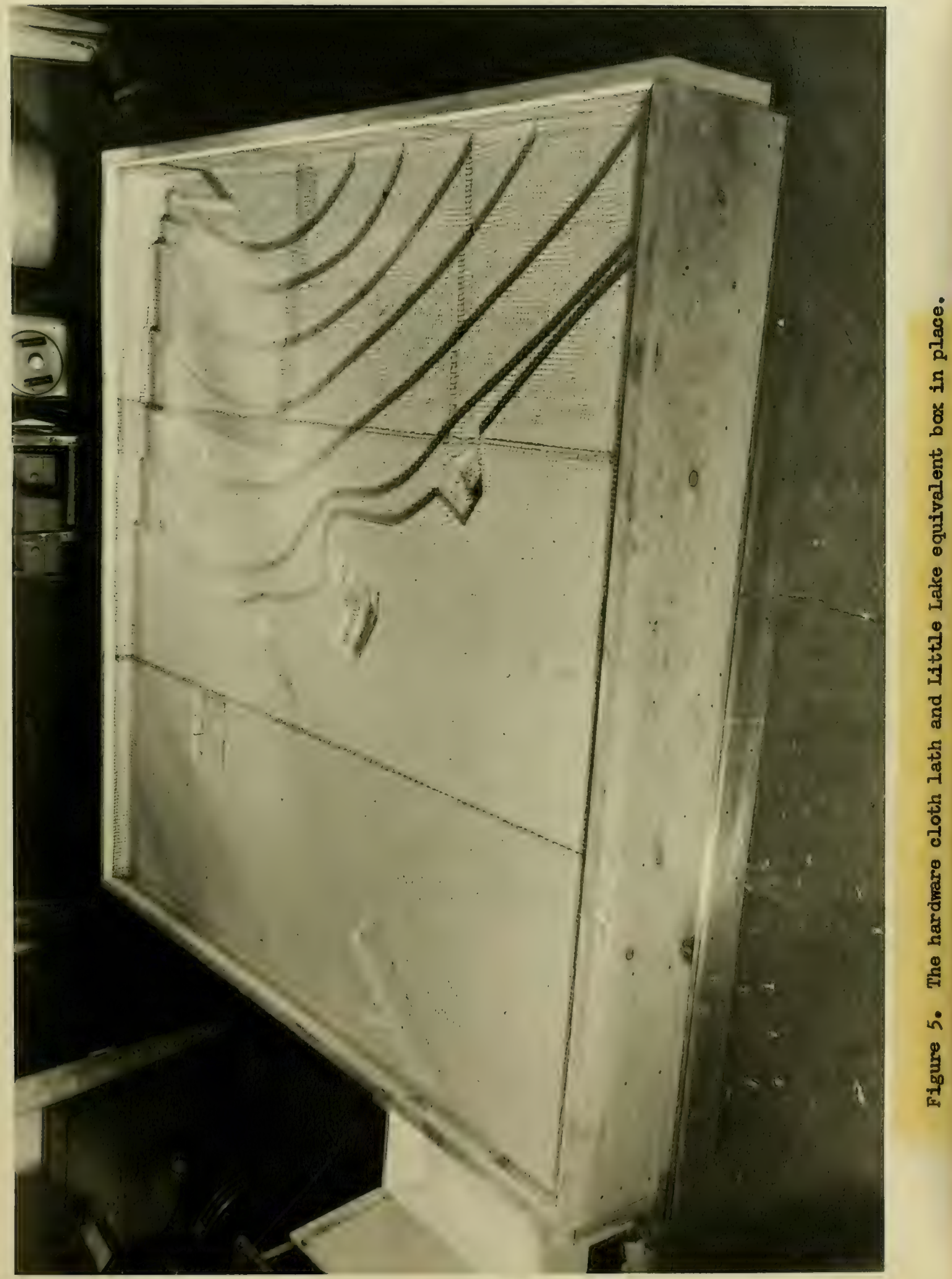





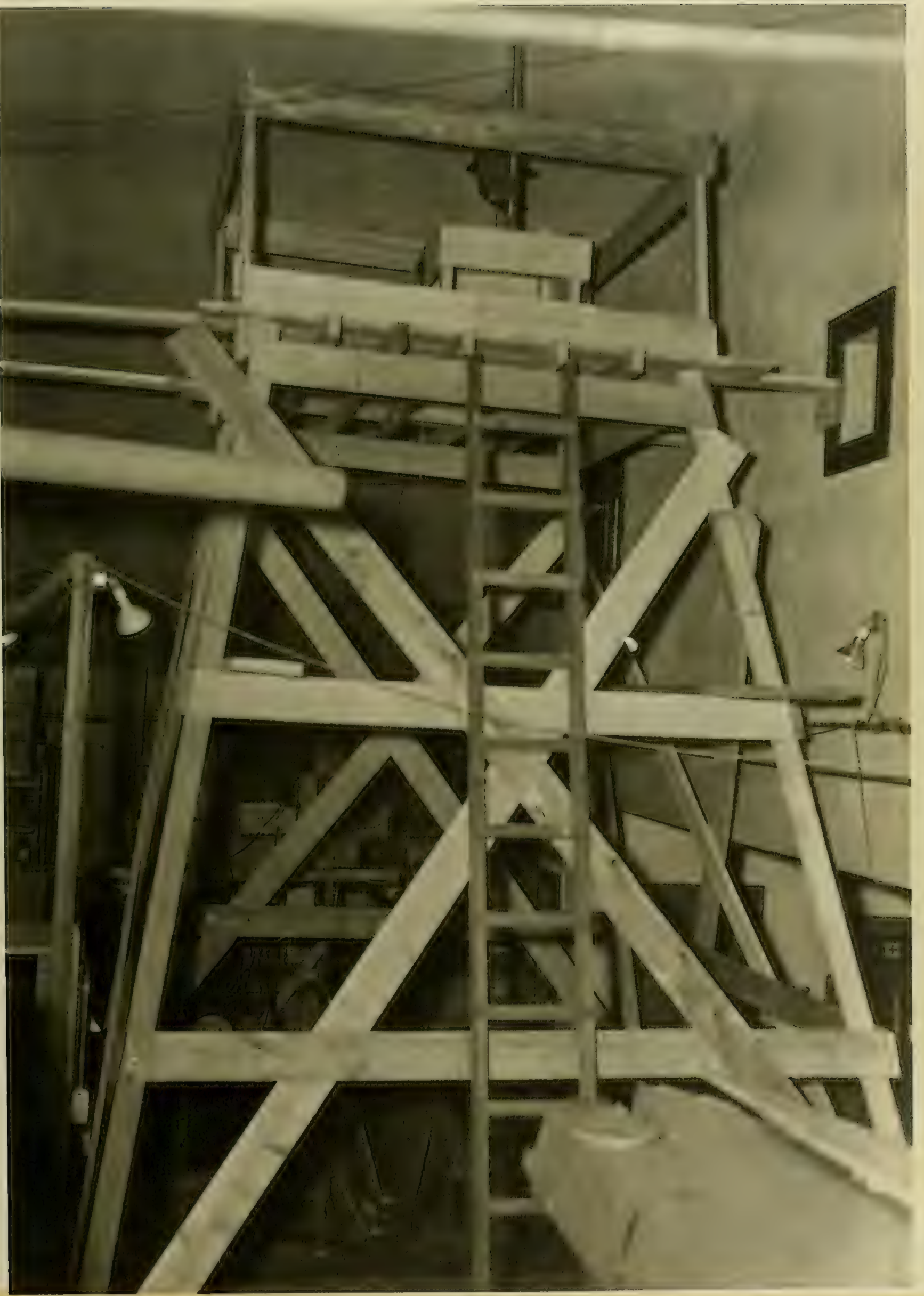

Figure 6. The tower \& photographic platform 16 feet above modeled surface. 



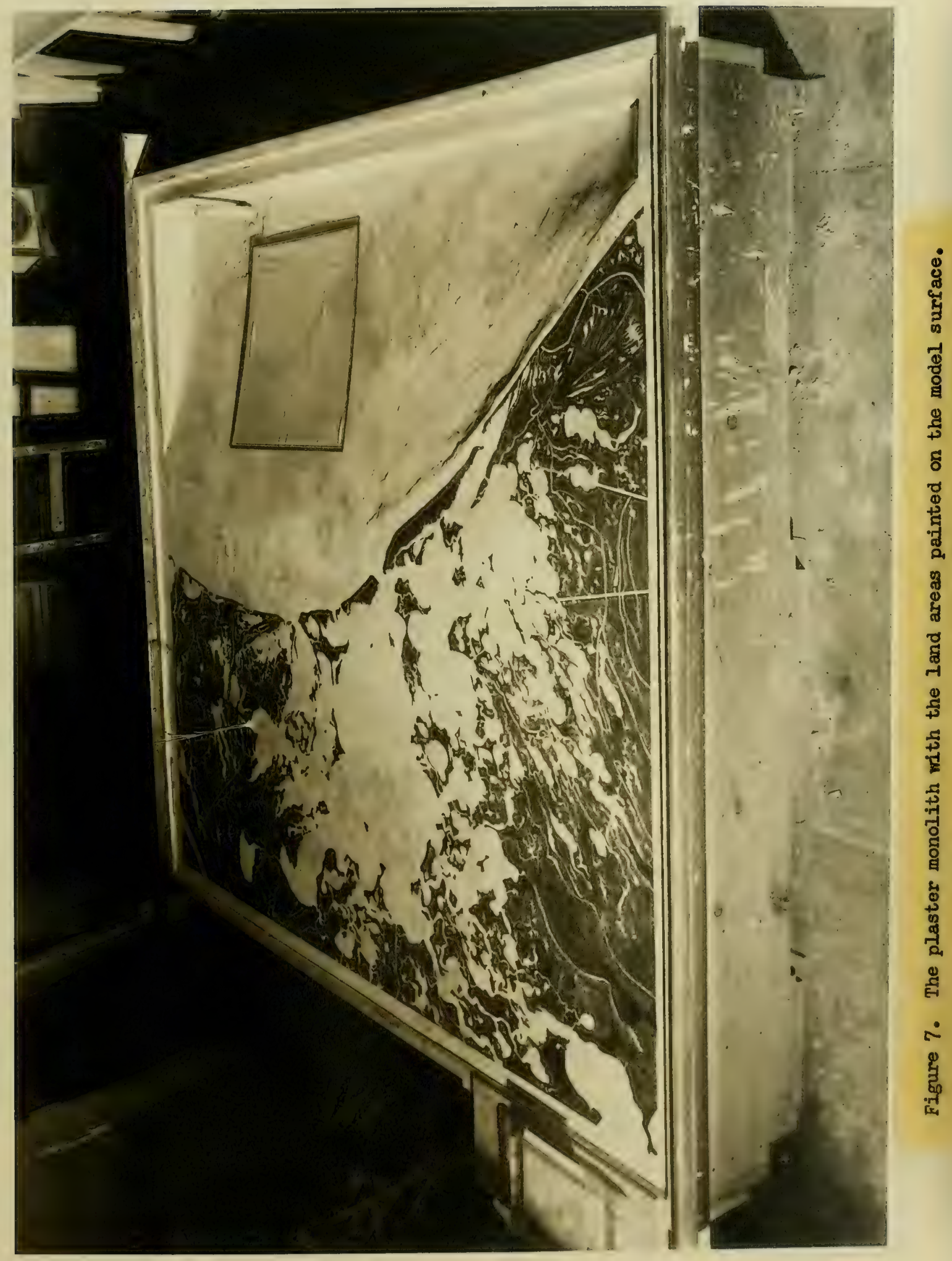





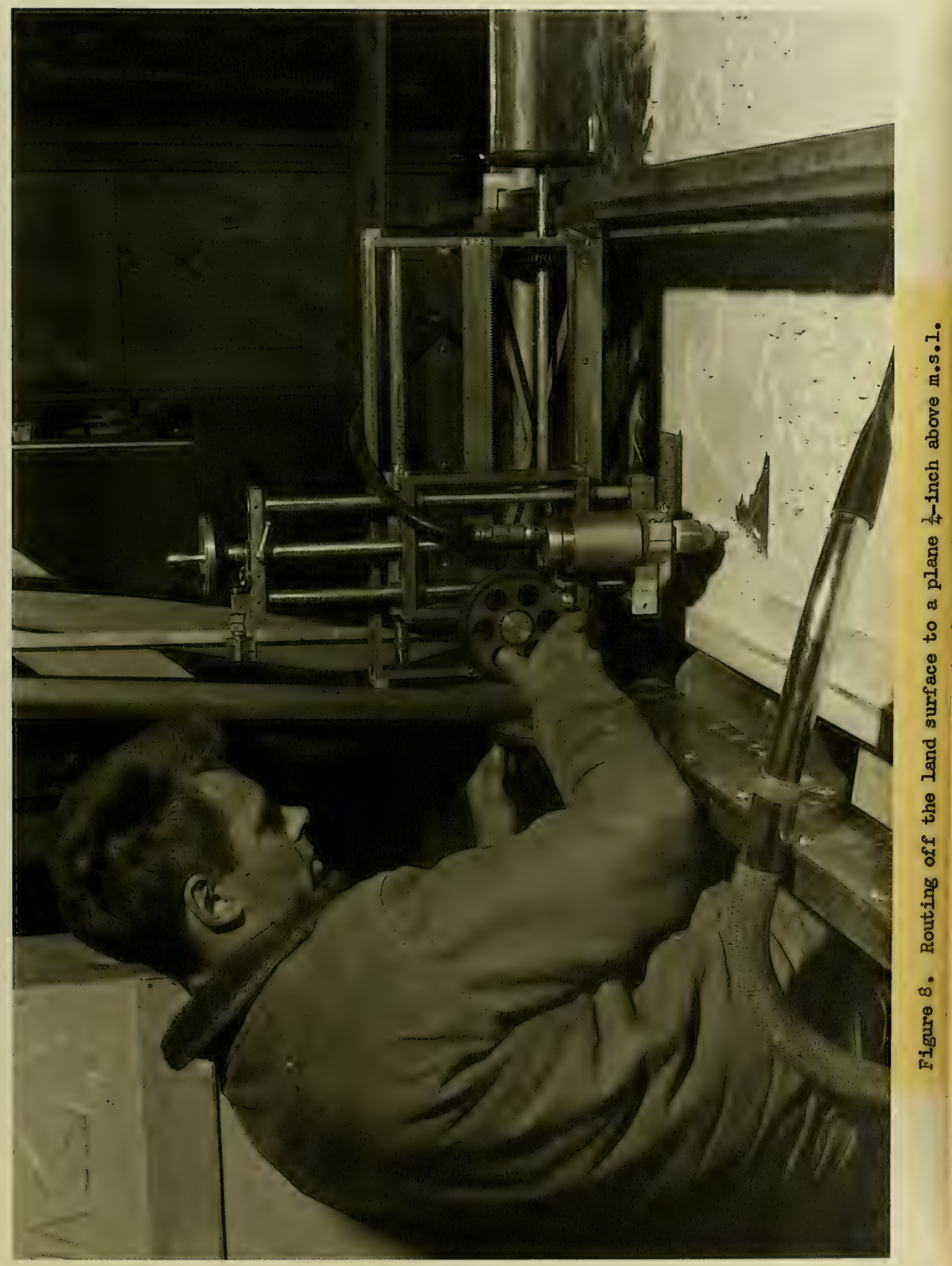





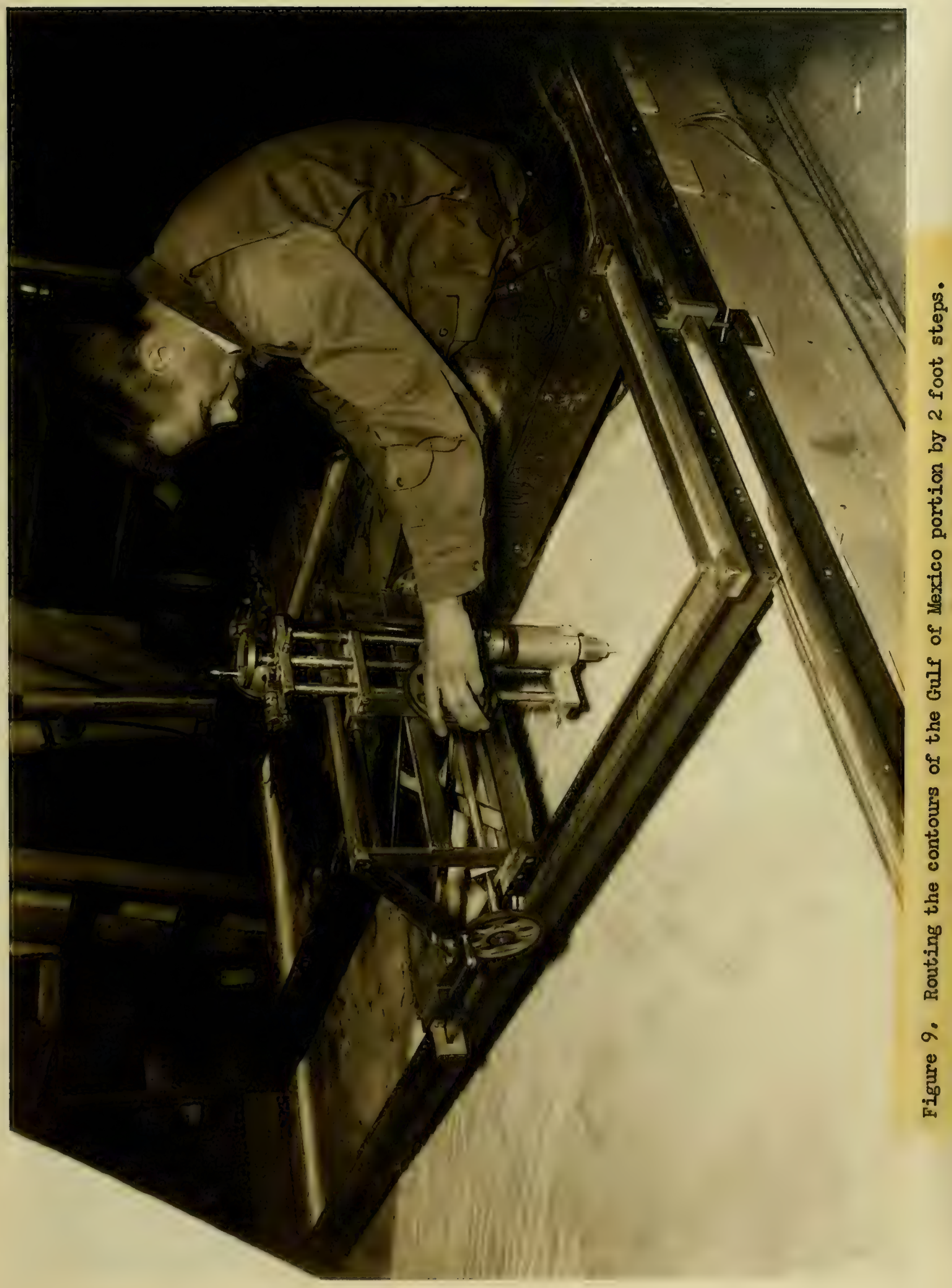





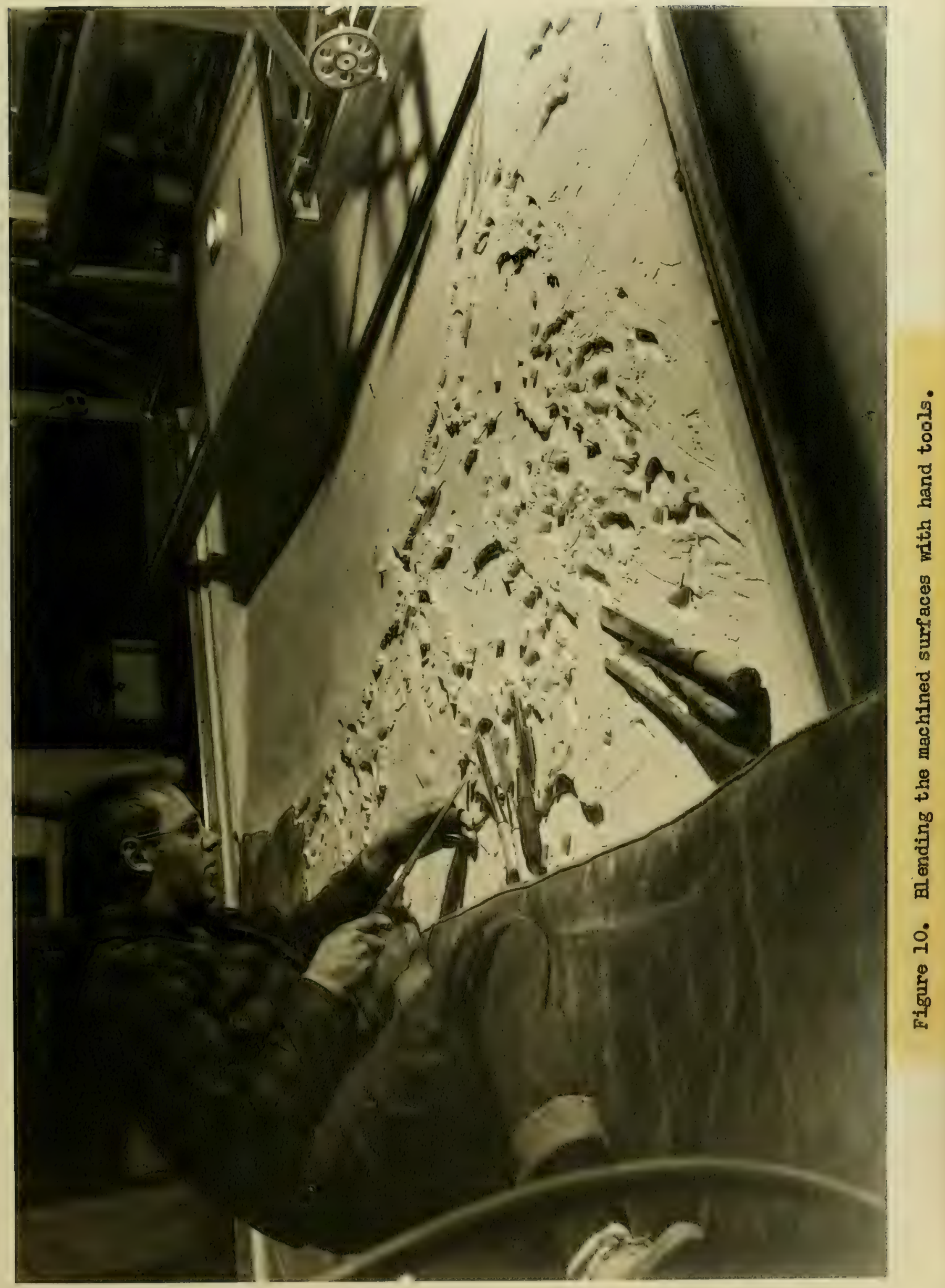





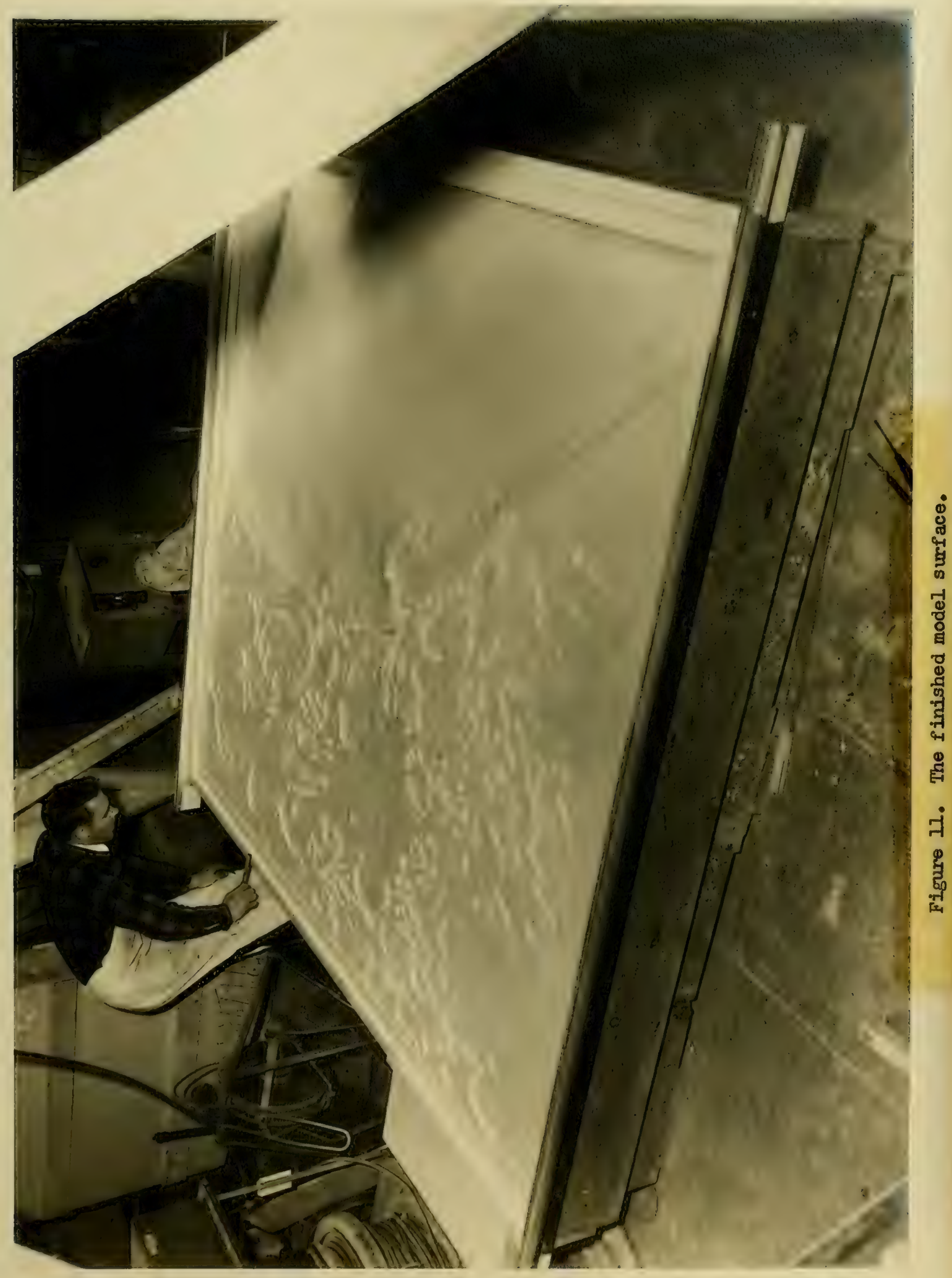


the flooded model, as many hydraulic occurrences as possible were compared with data from the detailed field reports of H. A. Mamer, and observations by V. H. Brogdon and the writer. With few adjustments of the model the times of slack water, the occurrence of high and low water, the particle excursions, and magnitudes of velocities were reproduced at a large number of points. Auxiliary equipment was designed to reproduce tides, winds, rainfall and industrial discharge scaled in accordance with the model constants.

The tide machine was composed of three drums, each of which displaced water and changed sinusoidally the water level in the model at rates in accordance with the time scale $1 / 1440$ (one minute per model "day"), Each drum was driven by a scotch yoke through a stroke of 2 inches; the semi-diurnal drum having a displacement determined by the sum of the amplitudes of the $\mathbb{M}_{2}, S_{2}$ and $\mathrm{N}_{2}$ tidal components and a speed number of the average of the component speed numbers, the first diurnal drum a displacement determined by the amplitude of the $O_{I}$ tidal component and a speed number of the $O_{1}$ component, and the second diurnal drum a displacement proportional to the sum of the $K_{I}$ and $P_{I}$ tidal components and driven at the

I/ The Tide in Barataria Bay, U. S. Coast \& Ceodetic Survey, Washington, D. C., July 1947.

The Currents in Barataria Bay, U. S. Coast \& Geodetic Survey, Washington, D. C., February 1948. 

average speed of these two which is precisely a mean solar day per $360^{\circ}$. Thus, the second diumal drum was run at one cycle per minute and the other two at 30.5 seconds and 64.5 seconds per cycle respectively. This combination produced a succession of great diurnal tides yielding to lesser semi-diurnal tides at intervals of about two "weeks," a progression characteristic of this region of the Gulf of Mexico (fig. 12).

The winds were generated by tank-type vacuum cleaner blowers mounted around the model at the cardinal and intercardinal points. Each was wired to a switch at a central control point and its rate of pumping governed by a Variac. Thus, it was possible to vary the wind speed and direction to reproduce either the detailed or average wind as it occurred during the periods of interest to the sponsors and in accordance with the records of the U.S. Weather Bureau reports from Grand Isle, Louisiana. Velocity calibration of the wind machines was accomplished by observing the rate at wich the surface layer of water in the model was propelled under wind stress at the steady state. It was assumed on the basis of field experience and values derived from empirical equations for wind stress that in more than 10 miles of fetch the stress on the surface is such as to produce an equilibrium surface current speed between 2 to $3 \%$ of the wind speed. By this

2/ See Appendix V attached to Copy No. 1 .

3/ Sverdrup, Johnson \& Fleming, The Oceans, Prentice Hall, New York, 1942, pp. 489-503. 

measure, it was possible to produce winds of hurricane force (100 "knots") from the simultaneous draught of three blowers running at full speed. Due to the small horizontal scale and the vertical exaggeration of the model, the capillary wave height was too small and the capillary wave length was too large for wave action to be in scale or to produce the significant mixing effects found in nature.

Rainfall, which amounts to 60 inches per year on a watershed of some 1100 square miles to the north of the modeled area, drains through the modeled area at the rate of approximately $450,000,000$ cubic feet per day, according to Marmer. This flow was introduced in the Little Lake equivalent box at the northwest end of the model by means of a pump attached to the tide machine. This pump drew water from the Gulf and produced both a closed circulation system for the rainfall, and a current of water through a venturi which generated a slow longshore current moving westward along the coast in agreement with known offshore circulation.

Industrial wastes (also referred to as bleed water) were introduced into the model through copper tubes embedded in the plaster and terminated at their proper geographical positions on the Freeport Sulphur Company properties. The injection ends of the tubes were attached to open glass cups at the level of the water in the model.

$4 /$ The rainfall on the modeled area was disregarded. 

Each cup was supplied at first by a calibrated dropper and later on by the outlet of a proportioning funnel, both of which in turn being fed by a $100 \mathrm{cc}$ hypodermic syringe acting as a positive displacement pump closed by a motor-driven screw. Thus, it was possible to know and control the quantities of industrial waste being introduced into the model either by counting drops delivered per minute from calibrated dropper tips or by timing the revolutions of the motor-driven screw. The industrial waste was simulated by an acid. The acid concentration chosen was such that when it mixed with a given number of volumes of ambient model water, containing an indicator solution of brom cresol green, it changed color from yellow to blue. The model water was set chemically to a known alkaline state (blue) such that for a given acid strength the color change (yellow-blue) would occur at a predetermined dilution. In this way the industrial waste, more concentrated than any specified dilution figure was represented by yellow water, and further dilutions in excess of those specified by a pleasing blue color which pervaded the whole model and varied in intensity with the depth of water.

Under certain conditions, continued discharge of industrial waste eventually extended unneutralized acid boundaries into the Gulf portion of the model. If routine titration of samples of water drawn from the Gulf showed it necessary, as the unneutralized acid reached and mixed away in the Gulf, a base of identical strength was introduced volume for volume into the Gulf at the 

circulating venturi. In this way the ambient water in the Gulf volume was rendered chemically infinite and was maintained quantitatively in its alkaline condition. Thus the model could be mun indefinitely to study the steady states of waste distribution in the bays under a variety of controlled conditions.

The results of the model studies were recorded automatically in color photographs made simultaneously with each of two cameras (1) a $35 \mathrm{~mm}$ Graflex pneumatic microfilming camera operated once every 7.5 seconds producing 8 exposures per model "day" and (2) a $16 \mathrm{~mm}$ Bolex time-lapse motion-picture camera operated electrically at I exposure every second yielding 60 exposures per model "day" and effecting a contraction of time, when projected at 16 frames per second, of $1 / 23040$, that is, of 1 model "day" per 3.75 mean solar seconds. Light for photography was provided by 12 No. 2 photoflood lamps in reflectors, a total of 6000 watts illumination in controllable quantities for uniform lighting. An electric clock fitted with a dial graduated in 24 model "hours" swept by the second hand, 60 "days" indicated by the minute hand, and 24 "months" indicated by the hour hand, was mounted in the field of view of both cameras so that the elapsed time from the beginning of each experiment was automatically indicated and totalized. Similarly the contribution of each of the tide drums was integrated and the sum of their effects indicated by a traveling pointer on a scale large enough for the cameras to record the height of water to 0.05 scale "foot." The same integrator was coupled to a pen 

which wrote a record of the tides on a paper tape moving at $1 / 2$ inch per minute ( $f i g .12$, and completely represented in Appendix IV of Copy No. 1 only). The wind direction was indicated by a small banner on a circular card placed on an island (unnamed) near the center of the model. In "no wind" conditions this indicator was removed from the model.

The tests of the model consumed approximately 65,000 model "hours" (about seven and one-half model "years") and were recorded in color on approximately 13,000 frames of $35 \mathrm{~mm}$ film strip and 3,000 feet of $16 \mathrm{~mm}$ time-lapse motion-picture film. The bulk of this record has been reduced to a series of 41 sumary drawings (Appendix III) representing the continuous and intermittent (tidal) excursion of the simulated industrial waste in the model for each wind condition in each of two major bleed water discharge conditions, Tests $B$ and $C$, and two concentration conditions, nominally $1: 1000$ and 1:200. Test $A$, which shows the distribution of fresh water drainage through the model area, is most easily seen in the original $16 \mathrm{~mm}$ time-lapse motion picture. However, figure 13 shows a characteristic stage after 30 "days" advance of fresh water into Barataria Bay from the north. This circulation of fresh water drainage undoubtedly tends to block the otherwise free circulation of water from Lake Grande Ecaille into Barataria Bay. 


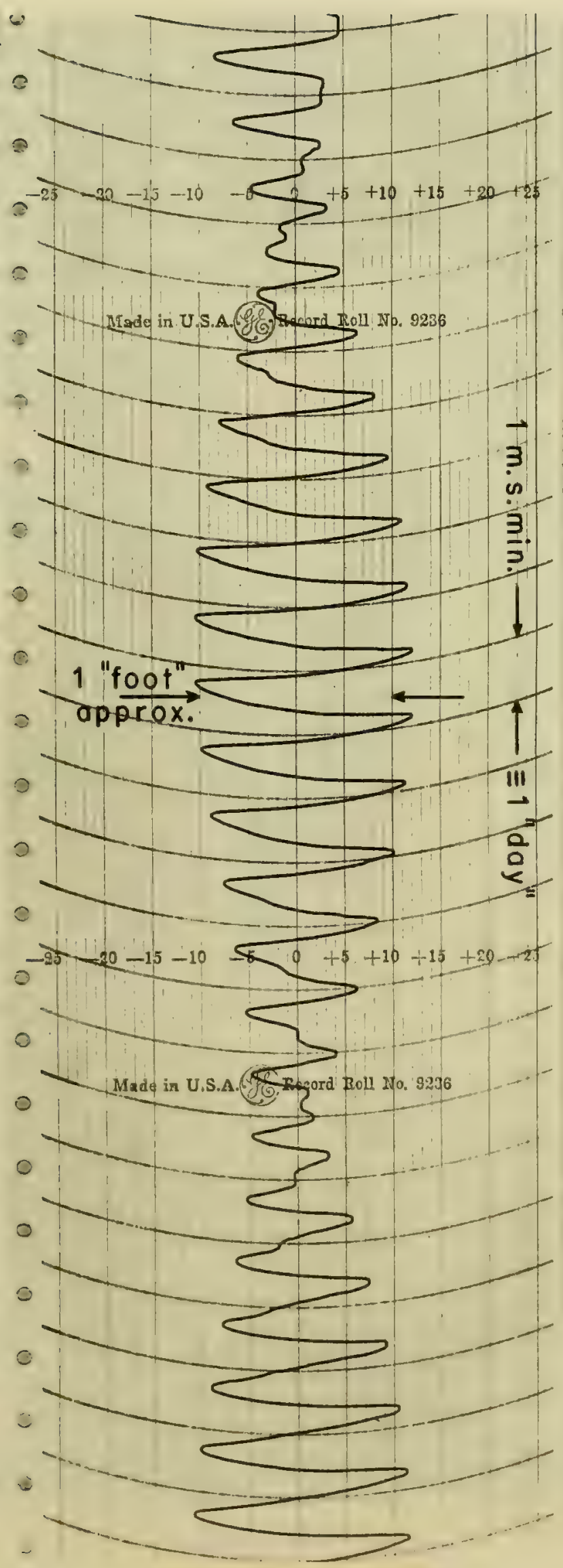

Figure 12. 



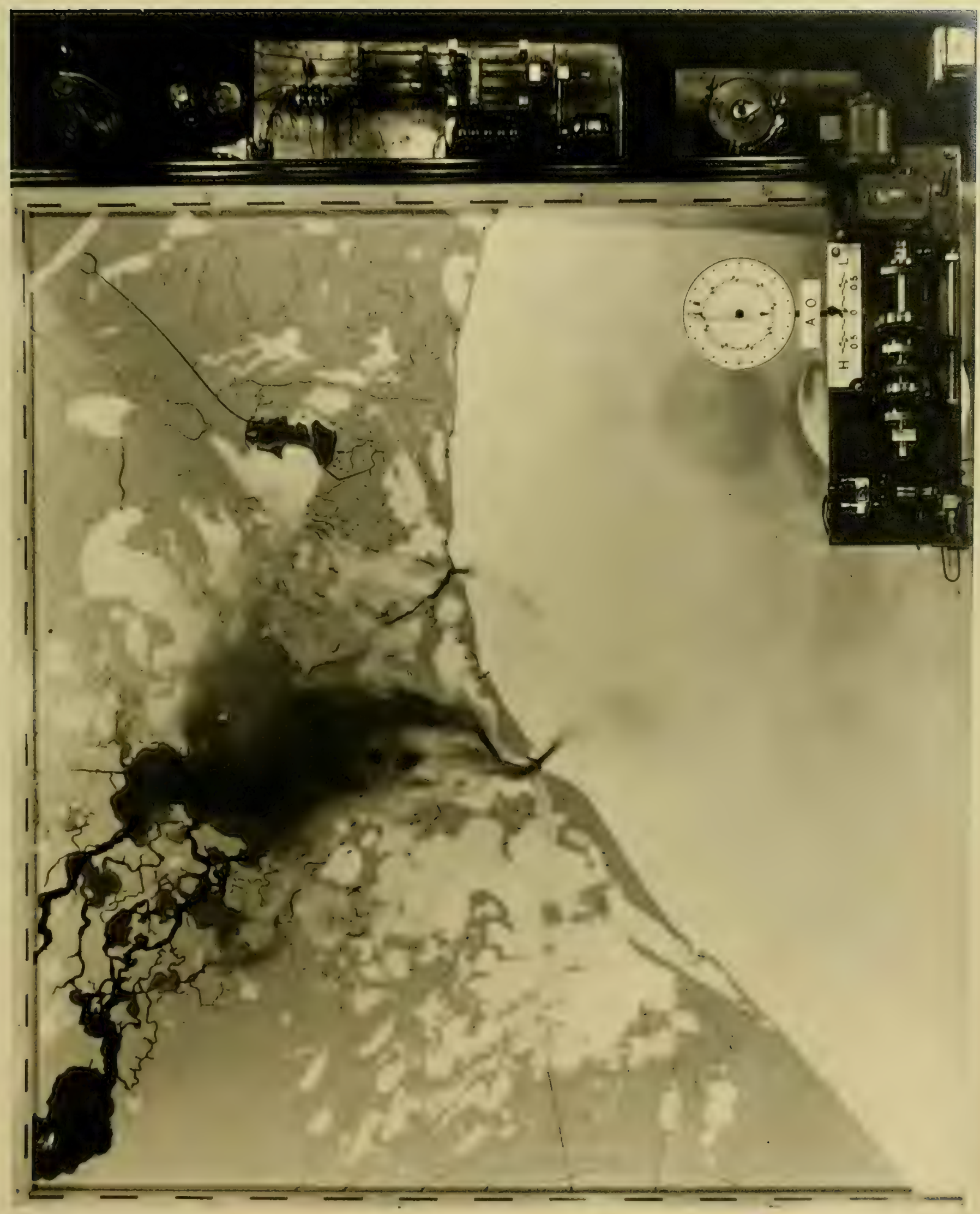

Figure 13.

Test AO after 30 days continuous rainfall discharge. 



\section{THE ROLE OF MODERN HYDRAULIC MODELS}

Hydraulic models as they are understood and employed today are the product of several decades of experimental development. There are no comprehensive mathematical relationships to express the entire range of correspondence between models and their prototypes. Lacking these, the design and operation of hydraulic models is an art and the results obtained are necessarily subject to interpretation. The process of interpretation is frequentiy qualitative or categorical in the "is" or "is not" sense since a full accounting of all the physical properties of a fluid system is not always possible. Within these limitations, however, the use of models has great interpretive value. Today, most important problems in fluid engineering are solved through combined consideration of model experiments and mathematical analys is of the known physical factors.

The function of a model is essentially that of an analogue computer. When a geometrically extensive or lengthy series of hydraulic events involves a great number of complex factors it is too difficult for the human mind and too laborious for exact mathematical analysis to cope with them all simultaneously. Usually, more intelligible results can be obtained if a similar system is constructed and studied under controlled laboratory conditions.

The degree of similarity between a model and its prototype 

may be only geometrical, or it may be kinematic, or dynamic. Two structures are said to be geometrically similar if the ratios of all homologous dimensions are equal. Kinematic similarity is similarity of motion. The motions of kinematically similar systems are geometrically similar and the ratios of the velocities of the several homologous particles in motion are equal. Few models are similar to a higher degree. It is possible, however, for two systems to possess dynamic similarity either in certain or all respects. In order to achieve dynamic similarity the two systems must be kinematically similar and in addition the ratios of the homologous masses and forces concerned in the motion must be equal. The condition of complete dynamic similarity is seldom achieved except in instances where the model is identical with the prototype in every respect. 



\section{THE SCALES OF THE BARATARIA BAY MODEL}

The scales of the Barataria Bay model are based primarily on the equations of kinematic similarity due to Froude. These equations concern the relationships between gravitationally energized fluid systems in a state of continuous quasi-hydrostatic equilibrium. In an estuary like Barataria Bay there are two dominantly gravitational factors controlling the circulation; the tides and the drainage of rainfall. Wind stress is also present and scaled to the model since it produces very significant water motions, but since this force is not gravitational its scaling transcends kinematic relationships.

Once the similarity of circulation in the main bodies of water is established in a model it is possible to introduce an indicator to trace the progress of suitably scaled quantities of industrial discharge under a variety of simulated natural conditions. This was the purpose for which the Barataria Bay model was built.

The notation used in scaling relationships is similar to ordinary physical notation except that all symbols that apply to the prototype are followed by a subscript "p" and those applying to the model are followed by a subscript "m." Scaling ratios are usually dimensionless and followed by a subscript "r" definod for any quantity $J$ as 



$$
J_{r}=\frac{J_{m}}{J_{p}} .
$$

It is possible to assign $J_{m}$ unit value and express $J_{p}$ as its multiple. Thus the time scale in a model compressing the events of one mean solar day within the compass of one mean solar minute; $T_{r}=T_{n} / T_{p}=1 / 1440$. The units of the ratio cancel mathematically but if they are preserved the scaling ratio expresses, instance for instance, the proportion in common units of the homologous quantity in either the model or the prototype. In the case illustrated one may justly speak and think interpretatively of a model "day" but this interval is still only one mean solar minute for purposes of calculation.

Choice of the vertical and horizontal scales was determined for the Barataria Bay model by the dimensions of the available space, the vertical distortion required to maintain a useful depth of water and by the rate of going of the model. Because of the complexity of the water-land areas in the Barataria Bay region it was considered imprudent to build the model on a horizontal scale smaller than $1 / 20,000$. This horizontal scale allows all the area of interest to the sponsors and the additional areas containing significant influences of oceanographic and hydrologic kinds, except part of Little Lake, to be included in a space eight by eight feet. The remainder of little Lake was represent ed by a box of equivalent area mounted on the outside of the model at 

the northwest corner and connected to the fluid system by a tunnel of suitable-dimensions. The horizontal scale being thus fixed, and considering the desirability of a time scale of one mean solar minute per mean solar day, the vertical scale was calculated from the kinematic formula of Froude for the distorted open channel model in which the value of $g$ and properties of water are substantially the same for the model and the prototype.

$$
y_{r}=\left(\frac{I_{r}}{T_{r}}\right)^{2}=\frac{1}{193}
$$

This vertical scale is within half of one per cent of $1 / 16$ inch per "foot" and was so adopted. The resulting vertical distortion is

$$
D_{v}=\frac{I_{r}}{y_{r}}=\frac{192}{20,000}=\frac{1}{104}
$$

This amount of vertical distortion is acceptable.

The Froude velocity scale is derived from consideration of the rate of progress of a gravity wave of very great length compared with the depth of water; that is of tidal dimensions. The rate of progress of such a wave is given by $\sqrt{g d}$, where $g$ is the acceleration of gravity and $d$ is the depth of water. The acceleration of gravity is substantially the same in both the model and prototype hence the velocity ratio is entirely controlled by the 

square root of the vertical scale, $\sqrt{y_{r}}$. Thus

$$
V_{r}=\sqrt{y_{r}}=1 / 13.9
$$

This means that a velocity of $I$ knot in the prototype is represented in the model by a velocity of $1 / 13.9 \mathrm{knot}$ or $3.65 \mathrm{~cm} / \mathrm{sec}$. Since the available data on the velocities of flow in the prototype were given in knots, a ruler was constructed and graduated in $3.65 \mathrm{~cm}$ intervals with tenths on one side and quarters on the other so that the velocities in the model might be checked with these lengths against the seconds beat of a break-circuit chronometer made audible through a loud speaker.

With the establishment of the time and length scales it was possible to calculate the discharge scale for the rainwater and bleed water. The kinematic discharge of rivers and industrial waste is scaled with regard to volume $\left[\left(L_{r}\right)^{2} y_{r}\right]$ per unit time $\left(T_{r}\right)$. Since $T_{r}=I_{r} / \sqrt{y_{r}}$ the total discharge scale $Q_{r}$ is

$$
Q_{r}=\frac{\left(I_{r}\right)^{2} y_{r}}{\left(\frac{I_{r}}{\sqrt{y_{r}}}\right)}=I_{r}\left(y_{r}\right)^{3 / 2}=\frac{1}{5.3 \times 10^{7}}
$$

The discharge $Q_{m}=Q_{r} Q_{p}$ is the discharge into the model over the same interval of time as is expressed for the discharge in the prototype. To make this perfectly clear, if the discharge of a river is $\left(10^{n}\right) \mathrm{m}^{3} /$ mean solar day, the scaled discharge into the model must be $Q_{r} \times 10^{n} \mathrm{~m}^{3} /$ mean solar day, not per model "day." 

The characteristic kinematic scaling factors used in the Barataria Bay model construction and operation are as follows:

\begin{tabular}{|c|c|c|}
\hline$I_{s}$ & horizontal length scale & $1 / 20,000$ \\
\hline $\mathrm{y}_{\mathrm{r}}$ & vertical length scale & $1 / 192$ \\
\hline$D_{v}=L_{r} / y_{r}$ & $\begin{array}{l}\text { vertical distortion } \\
\text { factor }\end{array}$ & $192 / 20,000=1 / 104$ \\
\hline$T_{r}=I_{r} / \sqrt{y_{r}}$ & time scale & $1 / 1440=1 \mathrm{~min} \cdot /$ "day" \\
\hline$V_{r}=\sqrt{y_{r}}$ & velocity scale & $1 / 13.8(3)=3.65 \mathrm{~cm} / \mathrm{sec} /$ "kt" \\
\hline$Q_{r}=I_{r}\left(y_{r}\right)^{3 / 2}$ & discharge scale (total) & $1 / 53,000,000$ \\
\hline sts & kinematic density scale & $1 / 1$ \\
\hline $.025 \mathrm{~V}_{\mathrm{r}}$ & wind scale & (empirical calibration) \\
\hline
\end{tabular}

The approximate physical dimensions of the Barataria Bay model are given to provide some acquaintance with the scale of the model experiments. About six months of effort by sixteen part- and fulltime craftsmen were required to build the model and its auxiliary equipment.

over-all north-south length of modeled surface over-all east-west length of modeled surface maximum depth in Gulf portion

Characteristic depth in Barataria Bay/-Grande Ecaille portion height of land above msl. material routed away to form modeled surface volume of water in system weight of flooded model dimensions of model working space volume of model and auxiliary equipment electric motors (synchronous) electric motors (controllable speed) power consumption (lighting) power consumption (total mechanical)
8.0 feet

8.0 feet

0.5 feet

0.04 foot

0.02 foot

600 lbs.

20 cubic feet

$2000 \mathrm{lbs}$.

$20 \times 20 \times 20$ feet

3000 cubic feet

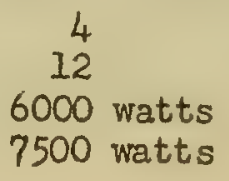





\section{RECONCILIATION OF KINEMATIC AND DYNAMIC PROCESSES}

The considerations preceding the table of scales have not included the scaling of density and wind velocity. The scaling of density influences mixing, which is essentially a dynamic process, and the scaling of wind velocity involves several considerations which are most accurately reconciled by an empirical process in the present state of the model making art.

5)

Density scales:- The circulation in estuaries frequently involves the mixing of two or more water masses of different density. In direct kinematic (Froude) scaling these density differences should be maintained $1 / 1$ in a model. The $1 / 1$ scale of densities leads to satisfactory results if the problem involves only the kinematics of incursion. If, for instance, a large mass of salt water flows in from the sea under fresh water as in a lagoon or estuary, the velocities, volumes and times of arrival of the main water masses are correct, but the mixing of the two water masses is usually quite dissimilar and unrealistic. The water masses tend to retain their identity too long in the model and to stratify. This result is inevitable, for a fractional scale kinematically similar model can rarely be made to possess a $1 / 1$ ratio of both

5/ While it is customary to consider density in model theory, it is usually simpler to measure specific gravity with respect to pure water at some standard temperature in experimental work. The specific gravity so measured is numerically equivalent to density. 

Froude and Reynolds numbers with its prototype. In the scales of oceanographic models the Reynolds numbers of flow in the model are of ten several orders of magnitude lower than corresponding values in the prototype.

In the Barataria Bay model two instances of deficient mixing are potentially present due to stratification accompanying (1) the density difference between the predominantly fresh drainage of rainfall into the northwestern part of Barataria Bay via Bayou St. Denis and Grand Bayou, and (2) the density difference between the ambient water of the Barataria Bay-Lake Grande Ecaille system and bleed water. Since there is at present no useful simultaneous solution to the problem of combined Froude and Reynolds density scales a choice was made in the first case in favor of the reciprocal Reynolds scale for increased realism of the circulation, and in the second to abide with the unmodified Froude scale since the injected volumes of bleed water exert a truly insignificant effect on the circulation.

The density difference between fresh water drainage and the ambient density of Barataria Bay water is amply sufficient to cause the fresh water to float on the surface of the ambient water as a thin but very extensive sheet. This tendency exists in nature to the same extent but it is overcome by the mixing processes associated with higher Reynolds numbers of flow, higher eddy diffusivity, and mixing by wave action.. In nature the several mixing processes carry the buoyant fresh water downward 


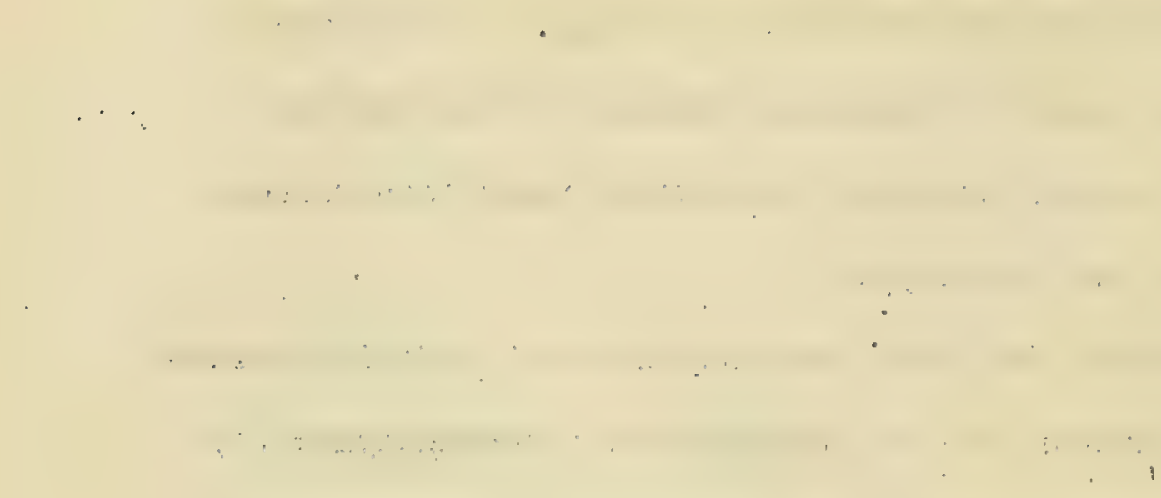

$\because$

, $\cdots$
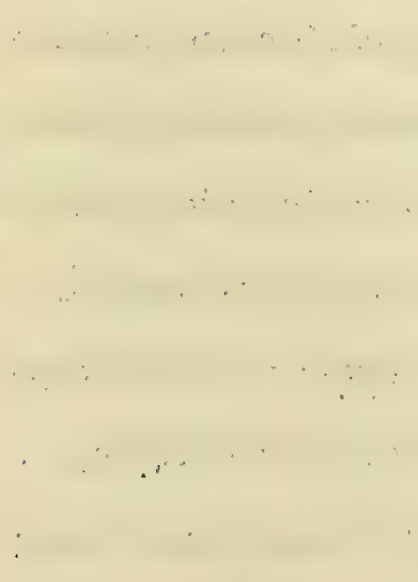
to form a homogeneous vertical mixture and are so vigorous that they also carry bottom silt upward in sufficient quantities to maintain a high degree of turbidity. In order to reproduce these effects in a model it is necessary to reduce the work to be done by the existing turbulence and associated eddy diffusion. The work to be done is lessened by reducing the density difference empirically by an amount roughly equivalent to the vertical distortion. The necessary change of density difference was actually found to be about two orders of magnitude. This factor reduces the initially small density difference $(0.012)$ to such an uncontrollably small magnitude $(0,00012)$ that the desired condition is more reliably represented by a homogeneous system. Thus the small volume (165 cc/min.) required for the rainfall contribution was pumped from the very large Gulf of Mexico volume and piped into the Little Lake equivalent box attached to the northwest corner of the model. This method of closing the rainfall circulation produced less than 1 part in 2000 error in the tidal circulation and automatically maintained a constant mean sea level.

In the second case, that of the bleed water density scaling, the volumes are insignificant compared with the tidal exchange of the waters they enter, and as bleed water is heavier than the ambient water it tends to sink. While bleed water is about as much heavier than the ambient water per unit volume as the rain water is lighter, and could also be well approximated by a homogeneous fluid, it was considered a more conservative approach if 

the $1 / 1$ density differences indicated by Froude scaling were maintained. This decision causes the simulated bleed water of the model to stratify and collect in depressions and practically to fill these depressions before traveling farther along. It also exaggerates the concentrations of bleed water by retarding its rate of mixing with the ambient water which would otherwise allow it to be carried away with greater rapidity and at greater dilution than the model indicates. When at length, under one-to-one density scaling conditions, the bleed water has reached the steady state of distribution and concentration and is mixing away at a rate equal to its rate of supply, the filaments of a given concentration are somewhat too long, too thin and somewhat too narrow because of the deficient Reynolds number of the flow and dissimilar eddy diffusivity of the gravity controlled kinematic model. It is significant, and a result of this choice, that the principal effect of wind on the bleed water tracer pattern in the model is to drive it upwind on the bottom. Both in nature and in the model there is a surface layer driven downwind which is usually somewhat thinner and more vigorous than the upwind counter current on the bottom. In nature wave action and more intense eddy diffusion would tend to mix vertically the contents of the two layers, that is, the bleed water accuralated in the bottom counter current

6/ Question concerning the advisability of departing from Froude scaling arises only in connection with models of very shoal estuaries wherein wave action reaches to the bottom and wind circulation is important. 
would be transported upward into the surface current and the uncontaninated surface water transported downward to dilute the concentrations present in the bottom counter current, and thus drive a more dilute solution of bleed water in both directions at once.

Wind velocity scales:- Wind stress on a nodel surface cannot be scaled from purely kinematic considerations because the factors concerned are primarily dynamic, and far from perfectly understood in nature. The transfer of energy by wind stress on the water surface involves considerations of at least the roughness of the interface, the temperature difference, temperature structures, eddy diffusivities, and densities of the two media, and probably the condition of the water surface skin. The surface roughness produced by simulated winds over small oceanographic models is usually too small due to the development of predominantly capillary waves rather than gravity waves. Capillary waves do not white-cap readily and they move faster as their lengths diminish while gravity waves move slower as their lengths diminish. Furthermore, to be in scale a modeled weve would have to have a scaled height $y_{r}$ and a scaled length $L_{r}$ in a distorted model. This latter requirement is often a physical impossibility. In view of all these difficulties it has been the practice to scale the model "wind" by scaling the translation effects it produces on the water surface. Oceanographic data indicate that where the fetch is greater than an equivilent of 5 to 10 thousand meters 

and a steady state exists, an appreciable thickness of the surface water will be moving at a speed in the order of 2 to 3 per cent of the steady wind speed. In the Barataria Bay model the average steady wind speeds in each of the cardinal and intercardinal directions were computed from data supplied by the Grand Isle station of the U.S. Weather Bureau. The blowers were driven at the proper rate to produce a $V_{r}$ scaled surface water motion equivalent to 2.5 per cent of the average wind speed wherever the fetch was great enough in each case. Every precaution was taken during the wind calibration and the model tests to have the model surface scrupulously clean so that the surface tension of the water skin maintained a uniform value and the rigidity of the skin was as low as possible. Before each test the surface was cleaned first by an ionic detergent and thereafter by a scummer. Tests were not begun until the detergent had had time to hydrolize and diffuse into the volumes of water below the surface. Since the action of the surface active agent is essentially monomolecular in thickness the resulting volume contamination was truly negligible.

The average wind velocities were calculated from statistics for the year 1946 prepared by the New Orleans Office, U. S. Weather Bureau, from observations made at Grand Isle, Louisiana, and are as follows: 



$\begin{array}{llllll} & \begin{array}{l}\text { Wind } \\ \text { direc- } \\ \text { tion } \\ \text { symbol }\end{array} & \begin{array}{l}\text { Wind } \\ \text { direc- } \\ \text { tion }\end{array} & \begin{array}{l}\text { Average } \\ \text { wind } \\ \text { speed } \\ \text { (knots) }\end{array} & \begin{array}{l}\text { Water } \\ \text { speed } \\ \text { (knots) }\end{array} & \begin{array}{l}\text { Varlac } \\ \text { (volts) }\end{array} \\ \text { Average spring-summer } & \text { WR } & 140^{\circ} \mathrm{T} & 4.0 & 0.10 & 32.5 \\ \text { Average southeast wind } & \mathrm{SE} & 135^{\circ} \mathrm{T} & 9.0 & 0.22 & 41.0 \\ \text { Average autunn-winter } & \mathrm{SR} & 085^{\circ} \mathrm{T} & 7.8 & 0.20 & 39.0 \\ \text { Average east wind } & \mathrm{E} & 090^{\circ} \mathrm{T} & 10.0 & 0.25 & 40.0 \\ \text { Average northeast wind } & \mathrm{NE} & 045^{\circ} \mathrm{T} & 10.0 & 0.25 & 47.0 \\ \text { Average north wind } & \mathrm{N} & 000^{\circ} \mathrm{T} & 11.3 & 0.28 & 42.0 \\ \text { Average northwest wind } & \mathrm{NW} & 315^{\circ} \mathrm{T} & 9.5 & 0.24 & 41.0 \\ \text { Average west wind } & \mathrm{W} & 270^{\circ} \mathrm{T} & 8.9 & 0.22 & 39.0 \\ \text { Average southwest wind } & \mathrm{SWT} & 225^{\circ} \mathrm{T} & 7.6 & 0.20 & 37.0 \\ \text { Average south wind } & \mathrm{S} & 180^{\circ} \mathrm{T} & 8.6 & 0.21 & 38.0\end{array}$

The direction of the average spring-sumer wind being within $5^{\circ}$ of southeast, the two directions were not distinguished and the higher average wind velocity was used as one test. The same distinction was omitted in the case of the average autumn-winter and the east wind which also differ in direction by $5^{\circ}$. Thus the actual wind velocities and directions used in the model tests were as follows:

$\begin{array}{llllll} & \begin{array}{l}\text { Wind } \\ \text { direc- } \\ \text { tion } \\ \text { synbol }\end{array} & \begin{array}{l}\text { Wind } \\ \text { direc- } \\ \text { tion }\end{array} & \begin{array}{l}\text { Average } \\ \text { wind } \\ \text { speed } \\ \text { (knots) }\end{array} & \begin{array}{l}\text { Water } \\ \text { speed } \\ \text { (knots) }\end{array} & \begin{array}{l}\text { Variac } \\ \text { (volts) }\end{array} \\ \text { "no wind" run to equilibrium } & \mathrm{O} & - & 0.0 & 0.00 & 00.0 \\ \text { Spring-summer equilibrium } & \mathrm{SR} & 140^{\circ} \mathrm{T} & 9.0 & 0.22 & 41.0 \\ \text { Autumn-winter equilibrium } & \mathrm{WR} & 085^{\circ} \mathrm{T} & 10.0 & 0.25 & 40.0 \\ \text { Northeast wind equilibrium } & \mathrm{NE} & 045^{\circ} \mathrm{T} & 10.0 & 0.25 & 47.0 \\ \text { North wind equilibrium } & \mathrm{N} & 000^{\circ} \mathrm{T} & 11.3 & 0.28 & 42.0 \\ \text { Northwest wind equilibrium } & \mathrm{NW} & 315^{\circ} \mathrm{T} & 9.5 & 0.24 & 41.0 \\ \text { West wind equilibrium } & \mathrm{W} & 260^{\circ} \mathrm{T} & 8.9 & 0.22 & 39.0 \\ \text { Southwest wind equilibrium } & \mathrm{SW} & 225^{\circ} \mathrm{T} & 7.6 & 0.20 & 37.0 \\ \text { South wind equilibrium } & \mathrm{S} & 180^{\circ} \mathrm{T} & 8.6 & 0.21 & 38.0\end{array}$



The first three of these runs probably have greatest significance because they show first the action of the tides and rainfall in distributing the bleed water and then how the average conditions of the two major seasonal wind directions and forces influence this distribution. In nature the wind over Barataria Bay apparently does not often blow long enough from any of the nonprevailing directions to re-establish a corresponding equilibrium distribution of bleed water. Each of the wind runs in the model was carried through a little more than one semi-monthly change of the diurnal tide sequenced The equilibria represented in the films of the model undergo continuous cyclic modification as the contributions of the tidal, rainfall and wind circulation systems change in relative importance through a two-week period. The drawings of the results of these tests (Appendix III) show the accumulated greatest extens ion of bleed water pattern and do not represent the distribution at any one time. Distinction has been made between the continuous and intermittent distribution, some of which is tidel and some due to the changing direction and force of the winds. 



\section{THE TDE MACHINE}

The characteristic circulation in an estuary such as Barataria Bay is directly related to the daily and monthly sequence of tides. The design and calculations of the tide machine were taken from broader considerations than the tides observed in the estuary itself.

For proper representation of tidal currents in open coastline models the tide machine should be located offshore in the modeled portion of the sea. If possible it should be equidistant from all parts of the model coast line so that the cotidal lines of the wave will be initially parallel to the coast and thereafter altered by refraction as in nature. If it is known that the cotidal lines lie initially at some angle to the coast or are normal to it describing a progressive wave moving up or down the coast line, the tide machine and barriers should be located so as to simulate this approach. The more remote the position of the tide machine from the modeled embayment the more changed are the amplitudes of the tidal coefficients of the machine as they are amended to apply at the position offshore. Fortunately this amendment often allows simplification of the tide generator, for some of the important complicating constituents in the tidal records near the openings to estuaries are due to the behavior of a simple wave inside the estuary. Since the modeled estuary will provide these complicating effects of its own accord, they 

should not be introduced twice. A good approximation of the final form of the offshore tide generator components can be obtained from a weighted average of the tidal coefficients at stations offshore or some distance away in both directions along the coast. The complexity and rate of going of a tide generator varies with the character of the tidal trace both within and along the seaward margins of the prototype. The components of a tide machine are determined from the relative importance of each of the several tidal coefficients compared with their sum. Unimportant coefficients of the same tidal species can be collected and introduced as a single component driven at the weighted mean rate. Important species having speed numbers sufficiently close to this weighted mean can be added as well to simplify the mechanism. Separate components must be set apart if their amplitudes and speed numbers are such that they largely determine the characteristic sequence of tides. In middle latitudes near open sea coasts, the tides are predominantly semi-diurnal with more or less diurnal inequality and a semi-monthly sequence of ranges. If neither the diurnal inequality nor the semi-monthly range sequence is large, the tide can be closely approximated by a single drum of the proper displacement driven at the weighted mean $r$ ate of all the significant semi-diumal species known for that station. If the diurnal inequality is too great to be neglected but the diurnal species are still unimportant, two semi-diurnal drums will suffice. If the diurnal species are important, and have a 

strong semi-monthly sequence of amplitudes, or if the diumal species are less important but not negligible, three drums will be required to produce an acceptable approximation of the tides. This is the case applicable to Barataria Bay.

The volume of water to be displaced in the model to effect the rise and fall of tides is the volume ratio $\left[\left(I_{r}\right)^{2} y_{Y}\right]$ times the volume change (tidal prism) in the flooded areas of the prototype. To calculate the displacements of the tide machine drums it is possible to prorate the scaled volume of the tidal prism among the important harmonic constituents in proportion to the ratio of their amplitudes to the sum of all terms. This process automatically includes and distributes the minor contributions of the neglected components.

The basic data for the Barataria Bay tide machine were taken from

1. Tidal Harmonic Constants (Atlantic Ocean) TH-I Jan. 1942

U. S. Department of Commerce, Coast and Ceodetic Survey

2. Tide Tables (Atlantic Ocean) Ser. 671, 1946.

U. S. Department of Commerce, Coast and Geodetic Survey

The two stations Pensacola, Florida and Galveston, Texas distantly flanking Barataria Bay were chosen as a combination which would be free of conspicuous estuarine resonance and the average signatures of which would be indicative of the characteristic tidal oscillations on the proximal parts of the continental shelf of the Gulf of Mexico. The average of the two sets 
of harmonic coefficients was taken as a first approximation of the open water tidal condition near the coast.

\begin{tabular}{ccccccccc} 
& $\mathrm{K}_{1}$ & $\mathrm{O}_{1}$ & $\mathrm{P}_{1}$ & $\mathrm{Q}_{1}$ & $\mathbb{M}_{2}$ & $\mathrm{~S}_{2}$ & $\mathrm{~N}_{2}$ & $\mathrm{~K}_{2}$ \\
$\begin{array}{c}\text { Pensacola } \\
\begin{array}{c}\text { (1934) } \\
\text { Galveston } \\
\text { (1939) }\end{array}\end{array}$ & 0.43 & 0.38 & 0.13 & 0.08 & 0.06 & 0.02 & 0.01 & 0.02 \\
\cline { 2 - 8 } & 0.35 & 0.10 & 0.08 & 0.31 & 0.10 & 0.08 & 0.01 \\
\cline { 2 - 8 } & 0.39 & 0.85 & 0.23 & 0.16 & 0.37 & 0.12 & 0.09 & 0.03 \\
& 0.43 & 0.12 & $\underline{0.08}$ & 0.19 & 0.06 & 0.05 & $\underline{0.01}$
\end{tabular}
Total range 1.24 feet

The smaIl $Q_{1}$ and $K_{2}$ constituents were neglected as individuals and their contributions incorporated in the total displacement of the tide machine drums. Three drums were built and driven through a stroke of 2 inches at the following speeds:

$$
\text { prototype model range radius displ. }
$$

$\mathrm{K}_{1} \mathrm{P}_{1}$ drum $24.00 \mathrm{hrs} 60.0 \mathrm{sec} .0 .51$ "ft." $5.35 \mathrm{in.} 181.0 \mathrm{in}{ }^{3}$

$\mathrm{O}_{1}$ drum $25.82 \mathrm{hrs} 64.5 \mathrm{sec} .0 .43$ "ft." $4.92 \mathrm{in.} 152.2 \mathrm{in.} 3$

$\mathbb{N}_{2} \mathrm{~S}_{2} \mathrm{~N}_{2}$ drum $12.21 \mathrm{hrs} 30.5 \mathrm{sec}$. 0.30 "ft." $4.10 \mathrm{in.} 106.0$ in. ${ }^{3}$

$$
\text { sum of all contributions } 1.24 \text { "feet" } 440.2 \text { in. }^{3}
$$

The final tests of this tide machine revealed errors of $3.2 \%$ in

I/ see following page. 
range and $15 \%$ in phase progress due to neglect of the longer period I

terms. The $M_{f}$ and $M_{m}$ constituents were partly accounted for in

rate adjustment of the $\mathrm{O}_{1}$ and $\mathrm{K}_{1} \mathrm{P}_{1}$ drums.

$Z / K_{1}=$ lunar declinational diurnal constituent, speed $=15^{\circ}, 041 / \mathrm{m} . \mathrm{s} . \mathrm{hr}$.

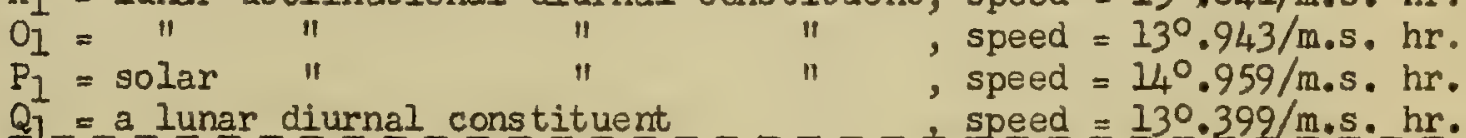

$\mathbb{M}_{2}=$ principal lunar semidiurnal constituent, speed $=280.984 / \mathrm{m} . \mathrm{s} . \mathrm{hr}$. $\mathrm{S}_{2}=$ principal solar semidiurnal constituent, speed $=30^{\circ} .000 / \mathrm{m} . \mathrm{s} . \mathrm{hr}$. $N_{2}=$ larger lunar elliptic " " , speed $=28^{\circ} .440 / \mathrm{m} . \mathrm{s} . \mathrm{hr}$.

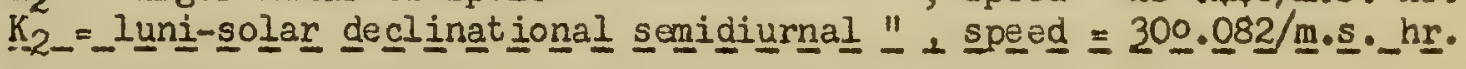

$\mathbb{M}_{\mathrm{f}}=$ lunar fortnightly constituent $u_{m}=$ lunar montily harmonic constituent
, speed $=10.098 / \mathrm{m} . \mathrm{s} . \mathrm{hr}$. , speed $=00,544 / \mathrm{m} . \mathrm{s} . \mathrm{hr}$. 



\section{KINEMATIC VERIFICATION OF THE MODEL}

The effectiveness and reliability of an offshore tide machine in a model is best evaluated by comparison with the tide curves at the principal entrances to the estuary and against the accompanying velocities of flow and the times of slack with respect to high and low water in the prototype. These comparisons serve the additional purpose of verifying the kinematic similitude of the whole model:

The verification of the Barataria Bay model was considered consistent with the available data and acceptable when the sequences of times of slack water and maximum currents led or lagged the times of high and low water within one-half "hour" (1.25 sec.) of the times observed in the prototype by H. A. Narmer. Particle traverses were accepted when they agreed within $\frac{1}{4}$ "mile" of Marmer's predictions. Similarly the speeds and directions of the currents were accepted when they fell within less than one quarter "knot" of the speeds and $10^{\circ}$ of the directions reported by Marmer in his reports on THE TIDE IN BARATARIA BAY and THE CURRENTS IN BARATARIA BAY. Further data on the details of the circulation around the Freeport Sulphur Company property were obtained by the writer or were supplied to him by V. H. Brogdon of the Freeport Sulphur Company. Particular attention was paid to the traverse of particles in Barataria Bay and to the asymmetry of duration of flood and ebb between St. Mary's point and the mouth of Bayou St. 

Denis caused by the rain water drainage through the estuary. The latter is a distinguishing feature of the Barataria Bay circulation because it is indeed a river mouth of rather peculiar shape. Test $A$ is concerned with a detailed study of rainfall drainage and its effect in tending to exclude the waters of both Lake Grande Ecaille and Bay Ronquille from Barataria Bay proper. In the eastern areas there is known to be an unusual division of the directions of ebb currents in the southern part of the Lake Washington system which causes the flood and ebb currents to diverge. Ebb currents flow north in part of Lake Washingt on and south in the region nearer Bay Chaland and reverse on the flood. The position of this division in the model was found to be in good agreement with field observations. Through the expedient of interposing the 20 by 20 mesh screens in the seaward passes the calculated rate of going of the tide machine was made acceptable. The calculated fresh water discharge through the Little Lake equivalent box led to a correct phase and duration of flood and ebb currents with respect to high and low water at Barataria Pass. Insofar as it is possible to estimate the reliability of a model with respect to field observations, the Barataria Bay model probably possessed a kinematic figure of merit of about 8 on a scale of 10. Since good quality field observations are generally rated at about this same figure of merit, and they alone reveal the behavior of the prototype, the estimated correspondence of the model to the true behavior of the prototype must be between 6 
and 7 on a scale of 10. The higher figure is more likely in this case because the field observations were made by larmer and his colleagues, whose sagacity is widely acknowledged. 


\section{SPECIFICATIONS OF THE MODEL TESTS}

The construction and verification of the Barataria Bay model discussed up to this point was of a routine nature and followed established precedents in most respects. The principal purpose of the model was to reconstruct the probablo distribution of the maximum quantity of bleed water discharged at any time during the sulphur mining operations of the Freeport Sulphur Company. Toward this end apparatus was constructed which would discharge these maximum specifled volumes as traceable material into the model at specified points under continuous discharge conditions. In each case the aim of the experiment was to determine the steady state distribution of the tracer under each of several wind conditions and at each of two dilution figures, namely 1 part in 200 and 1 part in 1000 . The specifications for these discharge conditions were supplied by the Freeport Sulphur Company and are as follows:

Test A. Investigate the progress and distribution in the model of fresh water draining through the Barataria Bay estuary from the watershed to the north of Barataria Bay.

Test $B$. Investigate the distribution in the model of a tracer having theoretical kinematic similitude with specified bleed water and capable of showing dilution by the ambient water not in nominal excess of 1 part in 200 and 1 part in 1000 discharged at the points and at the rates given below: 

at point VI, 1.4 million gallons per 24 hours, specific 8) gravity $1.057^{\circ}$ at $60^{\circ} \mathrm{F}$,

at point VIII, 1.0 million gallons per 24 hours, specific $8 /$ gravity 1.024 at $60^{\circ} \mathrm{F}$.

Test C. Duplicate Test B with the following additions: Investigate the distribution in the model of a tracer having theoretical kinematic similitude with specified bleed water and capable of showing dilution by the ambient water not in nominal excess of 1 part in 200 and 1 part in 1000 discharged at the point and rate given below: at the head of the Freeport Sulphur Company dilution canal, 1.5 million gallons of bleed water per 24 hours, specific 8 ) gravity $1.055^{\circ}$ at $60^{\circ} \mathrm{F}$, add at the same point 40 million gallons of Lake Grande Ecaille water per 24 hours, specific gravity $1.012^{8}$ at $60^{\circ} \mathrm{F}$ drawn in the model from the geometrically similar point at which this dilution water is drawn into the pumps in the prototype.

8/ See discussion beginning at the bottom of page 39. 
VIII. CHEMISTRY, OF THE BLEED WH.TER TRACER

In order to meet the specified conditions of the model tests the bleed water was simulated by sulphuric acid of suitable strength and specific gravity and the ambient water by ordinary tap water to which was added a suitable quantity of sodium carbonate and a fraction of a gram of brom cresol green indicator per model volume of about 600 liters. This choice of solutions was made in order to reduce as much as possible any chemical reaction between the solutions and the gypsum (calcium sulphate) plaster of which the model was made and also to avoid alteration of $\mathrm{pH}$ of the embient water through acidification by atmospheric carbon dioxide.

Sulphuric acid introduced to simulate the discharge of bleed water at the specified points and rates in the model necessarily produced a yellow color change in the brom cresol green indicator in solution with it. The color of brom cresol green in alkaline solution is a pleasant blue and the color change as the pH rises through the range 4.0 to 5.6 is at first green and then bright yellow. The change is abrupt from blue to green and more gradual from green to yellow. As the sulphuric acid flowed with the circulation of the model a certain amount of mixing took place which diluted the sulphuric acid, and at the same time reaction took

* place with the carbonate ions present in the admixed ambient water. The mixing processes were mainly due to molecular diffusion and microturbulent exchange across the interface between 

the sulphuric acid solution and the ambient water. Thus there was a steep gradient of $\mathrm{pH}$ through this boundary and the color change of the indicator with $\mathrm{pH}$ was at first too abrupt for the individual stages of color to be discerned. As time passed and distance from the source increased the gradient between the acid and ambient alkali usually weakened sufficiently for the individual color changes to be observed. At length when the acid concentration was no longer sufficient to maintain a pH greater than 4.0 the acid became invisible, or neutralized. As pointed out earlier, mixing processes in the model were insufficient to represent the rate of mixing known from field evidence to exist in the prototype, and, since only mixing would permit the process of chemical neutralization to take place, the acid color of the bleed water tracer was generally maintained too long and too far from the source.

Generally speaking the concentration of a sulphuric acid and a sodium carbonate solution can be adjusted so that the intermediate color change from greenish yellow to green will take place when one volume of the acid is thoroughly mixed with any specified number of volumes of carbonate solution. One gram molecular weight of dilute sulphuric acid will be neutralized by one gram molecular weight of sodium carbonate in aqueous solution. Thus if a day's discharge of bleed water is represented by the discharge of a certain volume of liquid containing 0.01 gram molecular weight of sulphuric acid, the presence of this acid will be 
detectable through the yellow or green color of the brom cresol green indicator present until the acid has had an opportunity to mix and react with a volume of ambient water containing 0.01 gram molecular weight of sodiun carbonate. If the volume of 0.01 gram molecular weight of sulphuric acid solution is $1 / 1000$ as great as the volume of ambient water containing 0.01 gram molecular weight of sodium carbonate solution, the boundary across which the acid ceases to be visible represents a dilution of the acid by a factor of 1000 . Thus the color of the acid discharge was characteristic of $\mathrm{pH}$ lower than 4.0 and concentrations greater than 1 part in 1000, and invisible if its dilution exceeded this predetermined value. The same reasoning applies to the $I$ part in 200 dilution contour. During operation of the model, samples of the solutions concerned in the bleed water tracer system were titrated repeatedly to end points in both the green and yellow ranges. These values represented the greatest and least measurable dilutions respectively of the acid component and served to define numerically the dilution contours represented by color changes in the model.

The chemical titrations of simulated bleed water against ambient water were referred to a standard sodium carbonate solution of approximately 0.2 normal strength. Lin intermediate standard sulphuric acid solution of approximately 0.01 normal strength was prepared in order to monitor the concentration of sodium carbonate in the ambient water. The simulated bleed water 
solutions of sulphuric acid were made up in two liter quantities to a strength of approximately 0.5 nomal for the 1 part in 1000 tests and approximately 0.1 normal for the 1 part in 200 tests. The exact nomality of each solution was determined through comparison with the standard sodium carbonate solution. The standard sodium carbonate solution in turn was titrated against the intermediate standard acid solution. It was found that the acid prepared for the 1 part in 200 tests was 9.94 times as concentrated as the intermediate standard acid and that the acid prepared for the 1 part in 1000 tests was 50.2 times as concentrated as the intermediate standard acid. The entire mass of ambient water in the model was rendered suitably alkaline by addition of sodium carbonate. It was found that approximately 2.0 milliliters of the intermediate standard acid solution produced the blue-green to blue change of color in the brom cresol green indicator and that approximately 3.1 milliliters of intermediate standard acid produced the green-yellow color change when added to 50 milliliters of the sodium carbonate solution used as the ambient water. This ratio of concentrations was monitored by titrations throughout the nodel tests. During each test the dilution factor inevitably tended to shift. The average dilution factor was calculated for each run and the results of these calculations are tabulated in the log shoets and entered on the smooth plots of the results for each test. Two values are given; the value for the yollow- oreon change representing the highest measurable concentration of simuiated bleed 
water, and the value for the green-blue change representing the lowest measurable concentration of simulated bleed water. In each case it was the intention to make these values straddle the nominal dilutions specified for the tests. The dilutions were calculated as follows:

$\mathrm{V}_{\mathrm{g}}=$ volume of Gulf water titrated ( $50 \mathrm{cc}$ by volumetric pipette) $V_{a}=$ volume of intermediate standard acid used to neutralize $V_{g}$ $V_{b}=$ volume of simulated bleed water equivalent to $V_{g}$

$$
\begin{array}{ll}
\begin{array}{l}
\text { for } \\
\text { I:200 } \\
\text { tests }
\end{array} & \frac{V_{g}}{V_{b}}=9.96 \times \frac{V_{a}}{V_{b}} ; \\
\begin{array}{l}
\text { for } \\
\text { tests }
\end{array} & \frac{V_{g}}{V_{b}}=50.2 \times \frac{V_{a}}{V_{b}}
\end{array}
$$




\section{AUXILIARY PUMPING SYSTEMS}

The scaled quantities of bleed water were pumped through 1/8-inch copper tubes embedded in the plaster monolith. These tubes were terminated at point VI on the north bank of Humble Canal, point VIII on the west bank of the dredge cut, at the head of the dilution canal and on the shore of Lake Grande Ecaille adjacent to the head of the dilution canal (see fig. 14). The first three tubes were attached to a positive displacement pump driven by an electric motor through suitable screw. The fourth tube was used to withdraw the dilution volumes from Lake Grande Ecaille for return at the head of the dilution canal together with the appropriate volunes of simulated bleed water. Thus the hydraulic activities of the Freeport Sulphur Company mining operations having significance in these tests were substantially reproduced in the model.

The kinematic scaling factor of discharge $Q_{r}$ being $1 / 5.3 \times$ $10^{7}$ per mean solar day, the discharge per mean solar minute into the model is $Q_{m}=Q_{p} T_{r}$ or $1 / 7.68 \times 10^{10}$. One million gallons in the prototype is therefore represented in the model by a volume of 0.05 cubic centimeter of liquid or approximately one drop. Pumps that will deliver such small quantities of liquid per minute are difficult to build and to operate. In order to increase the volumes to be discharged by a single pumping unit, and to simplify the maintenance of predetermined differences of 

specific gravity, which in this case were more significant than specific gravity ratios, perrission was obtained to average the specified specific gravities of the simulated bleed waters and to maintain this average difference above that of the ambient water of specific gravity 1.000 at $60^{\circ} \mathrm{F}$ rather than 1.012 . Since specific gravity is more easily measured than density and is numerically equivalent to density if comparisons are made at a convenient standard temperature, usually $60^{\circ} \mathrm{F}$ or $20^{\circ} \mathrm{C}$, the resulting error in the equivalent density ratio was $1.2 \%$. The average difference in specific gravity of the simulated bleed water and ambient water at $60^{\circ} \mathrm{F}$ was taken as $1.045-1.012=1.033$ corresponding closely to that of a $5 \% \mathrm{NaCl}$ solution at the same temperature. Thus the kinematic density difference $0.033 \mathrm{gm} / \mathrm{cc}$, measured in terms of specific gravity under homogeneous temperature conditions equivalent to $60^{\circ} \mathrm{F}$, was adjusted by adding the necessary quantities of $\mathrm{Na}_{2} \mathrm{SO}_{4}$ to the bleed water tracer.

A positive displacement pump had been designed and built to handle the sum of both the simulated bleed water volumes and the dilution volumes, but later it was thought better practice to actually withdraw the dilution volumes from the model since these volumes form a small but possibly significant part of the local circulation. Relieved of this relatively major burden the bleed water pump had to be sun at very slow speeds, and during Test $B$ it was found that accurate proportional delivery of the scaled volumes could not be detected and controlled quickly enough to 
correct the "day-by-day" discharge rates but that the total discharge over a period of a "week" was acceptable. Since the points of discharge in Test $B$ are so close together and united in a common portion of the detailed circulation no significant error in the equilibrium distribution of bleed water could occur as a result of small proportioning errors. In Test C, however, a small proportioning error would be highly significant in the equilibrium distribution of bleed water because the dilution canal is part of a circulation pattern that is distinct from the Humble Canal system. Test $\mathrm{C}$ was, therefore, not run until a reliable rotary proportioning device had been built and installed. The total discharge of the bleed water pump was monitored as before by timing the revolutions of the driving motor shaft and screw. The results obtained in all bleed water discharge tests are believed to be representative and consistent in spite of the difficulties enumerated.

Two other pumping systems had significance in the model tests (1) the dilution system which withdrew scaled volumes of water from Lake Grande Ecaille and discharged them as a mixture with properly scaled volumes of bleed water added, and (2) the rainfall pumping system. The bleed water dilution system consisted of a small gear pump driven by a separate electric motor and monitored by counting the number of drops discharged per minute as they fell from a calibrated dropper tip. Since the scaled volumes involved a drop every few secands it was possible to observe the rate of 
discharge quite readily and maintain acceptable accuracy. Similarly the rain water circulation system involved the discharge of 165 cubic centimeters per minute, a volune equivalent to Marmer's estimate of the average fresh water drainage $\left(450 \times 10^{6} \mathrm{ft} .3 / \mathrm{day}\right)$ into Barataria Bay. This volume was pumped from the Gulf portion and was piped around the outside of the model to the Little Lake equivalent box where it discharged as a small steady stream. This flow was adjusted by means of a needle valve at the pump. The same punp also circulated a continuous stream in the Gulf of Mexico portion of the model through a venturi tube which entrained the Gulf water and produced the characteristic westward trend of the circulation of Gulf past the mouths of the passes into the Barataria Bay estuary. The venturi system was adjusted to produce a steady westward current of 0.25 "knot" along the coast line. 



\section{THE RELIABILITY OF THE MODEL TESTS}

The final figure of merit for all the observations represented in the model tests can be estimated only very roughly. The model itself is rated at 7 on a scale of 10 . The equilibrium distribution of bleed water in the model is estimated at the same figure. Hence the reliability of the bleed water tracer tests must be about 5 on a scale of 10. This figure of merit should be interpreted to mean that the contours of bleed water distribution may be overextended with respect to the actual occurrence in the prototype by a factor of 2 or they may be underextended by a factor of $1 / 2$. The kinematic density scale chosen in connection with simulated bleed water discharge caused the bleed water to sink unnaturally and inhibit the mixing processes that would otherwise dilute the discharge. With the condition of insufficient mixing applying so heavily to the first possibility and to the estimate of the figure of merit itself, underextension of the dilution contours of bleed water distribution is improbable. 


\section{XI. "FIRST GRRIVLLL" TESTS}

In addition to the tests for the equilibrium distribution of bleed water and penetration of fresh water discharge some separate tests were run and photographed to determine the minimum time required for a parcel of water composed of an hour's discharge from point VI in Humble Canal to travel to the head of Bay Ronquille. It was found that the volume of colored ink tracer released at point VI had to be deposited at a very critical moment near the beginning of a tropical ebb tide in order to reach the head of Bay Ronquille before the following flood carried it back toward its starting point. The minimum time required for a parcel to actually reach the head of Bay was approximately seven or eight hours .

Experiments in the field show that the travel time on the tropical flood tide from point VI in Humble Canal to Fisherman's cut on the east side of the Freeport Sulphur Company dredge cut is about five hours. The model confirms this result provided the tracer has neutral density. If the tracer is of kinematically (I/1) scaled density the discharge through Fisherman's cut is delayed until the dredge cut basin is filled and a stratified layer established which is almost level with the threshold of Billet Bay. Following this the travel time is the same for solutions of both neutral and kinematic density. 
Appendix I - Schedule of procedure in model tests.

Freliminary readiness check

1. check titre of ambient water and sea level, clean water surface

2. check specific gravities of ambient water and simulated bleed water for difference of 0.033

3. check rate of fresh water discharge at $165 \mathrm{cc} / \mathrm{min}$.

4. check filling of bleed water pumps with proper acid solution

5. check rate of discharge of bleed water pump

6. blow out bleed water discharge lines and flush with ambient water

7. fill bleed water discharge lines with proper acid solution to point of incipient discharge

8. check rate of going of tide machine

9. check tide recorder for ink and paper supply

10. check wind sock

11. check run code number

12. replace all photofloods and test lighting system, measure illumination

13. check power to wind machines, and alignments

14. check film supplies, cameras and exposures

15. check velocity of f'low through Barataria Pass, Bayou St. Denis

Operating duties

Chemist

1. keep running check and $\log$ of titre of ambient water

2. operate wind mackines on signal

3. place and remove wind sock on signal

4. change code numbers on signal

5. replace photoflood lamps as necessary

Mechanist

1. keep continuous watch on bleed water discharge rate, dilution pump rate, tide machine rate and fresh water discharge

2. start and stop and label tide recorder on signal

3. start and set elapsed-time clock on signal

4. replace photoflood lamps as necessary 


\section{Photographer}

1. keep continuous watch on framing, exposure, focus and film supply of all cameras, change film as required

2. keep watch on the over-all behavior of the model

3. general instructions for operating $16 \mathrm{~mm}$ time-lapse and 35 mm Graflex cameras

a. operate $16 \mathrm{~mm}$ time-lapse Bolex with $15 \mathrm{~mm}$ Eastman Cine f-2.7 objective and Type A Kodachrome in 100 foot rolls at the rate of $I$ frame per second

b. operate $35 \mathrm{~mm}$ Graflex Photorecord camera with $44 \mathrm{~mm}$ Wollensak $f-3.2$ objective at air pressure of 22 lbs using 100 foot rolls Type \& Kodachrome at the rate of 1 frame every 7.5 seconds. Synchronize with model clock

c. operate $35 \mathrm{~mm}$ Leica with $127 \mathrm{~mm}$ Wollensak objective f-4.5 at optional intervals during runs to equilibrium and at intervals of approximately one week during wind tests. Include the vicinity immediately adjacent to Freeport Sulphur Company area in field of view. Try to record significant changes or developments of the bleed water patterns.

4. make serial photographic record of model tests as follows:

a. run to equilibrium without wind - run both 16 and $35 \mathrm{~mm}$ cameras

b. run to summer wind equilibrium - mun both 16 and $35 \mathrm{~mm}$ cameras

c. return to no-wind equilibrium - no photographs

d. run to winter wind equilibrium - run both 16 and $35 \mathrm{~mm}$ cameras

e. return to no-wind equilibrium - no photographs

f. cardinal and intercardinal winds - start 16 and $35 \mathrm{~mm}$ cameras when equilibrium is attained, continue photographing until one-half monthly progression of tides has passed.

5. keep log of photographs and model behavior

Completion duties

1. secure all electrical machinery and lighting equipnent

2. flush model free of distributed acid

3. dry off land areas with sponges

4. secure work lights 


The pages that follow contain a chronological transcript of the photographic and chemical logs written during the model tests. In explanation of the various. symbols used to designate the tests:

1. The tests were designated $A, B$, and $C$; which designations connote briefly

Test A:- fresh water drainage through the estuary Test B:- bleed water discharge from points VI and VIII Test C:- bleed water discharge from points VI, VIII and from the dilution canal

2. Each of the $B$ and $C$ tests was run so that dilution of bleed water by ambient water would be shown at nominally 1 part in 200 and 1 part in 1000. These dilution factors are represented by the roman numerals $\mathrm{CC}$ and $\mathrm{Hi}$ respectively. 3. In addition, a separate combination of each of the $B$ and $C$ tests and each dilution factor was run under winds from the cardinal and intercardinal directions $\mathrm{N}, \mathrm{E}, \mathrm{S} . \mathrm{W}$, and $\mathrm{NE}$, $S E, S W, N W$ respectively with the qualification that $E$ was replaced by the average summer wind and labeled SR. Test A was run only under no-wind conditions and this and the phases of the other tests run without wind are labeled $\underline{0}$ in the wind column.

Thus a test designated BNEM, for example, means; test $\underline{B}$ discharge conditions, $\mathbb{N E}$ wind conditions, $\mathbb{M}$ or 1 part in 1000 dilution conditions. In general one test was run in one day. The time of day for each occurrence is given in local standard time and the elapsed time from the beginning of the model test is reckoned in model "days" of one mean solar minute duration. 


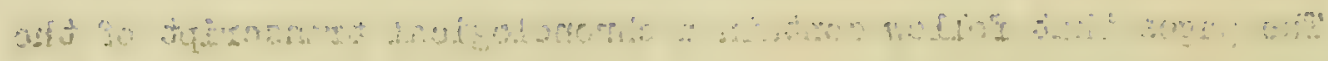

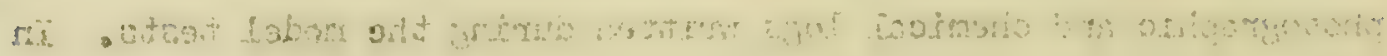

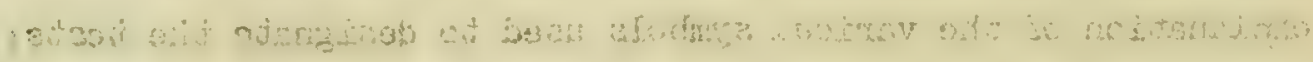

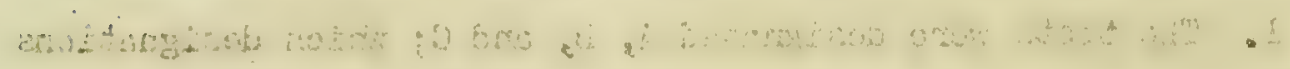

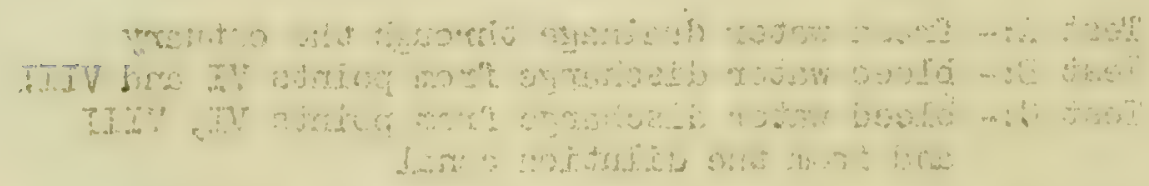

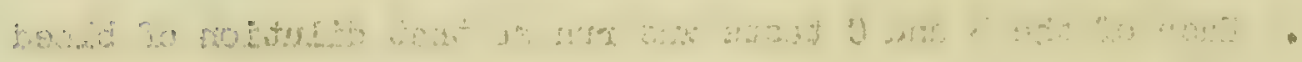

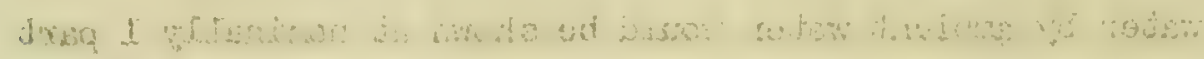

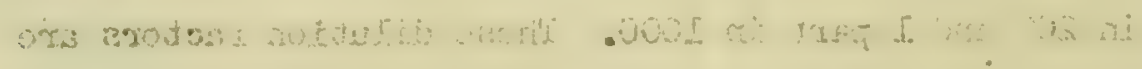

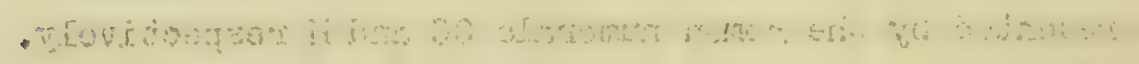

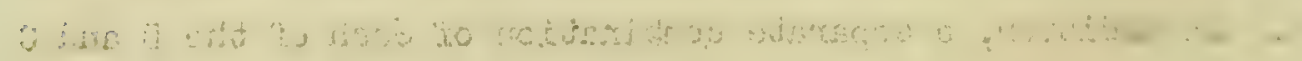

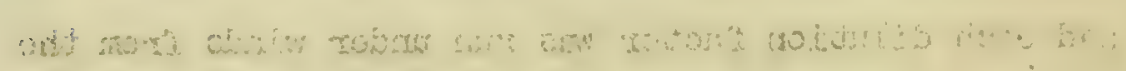

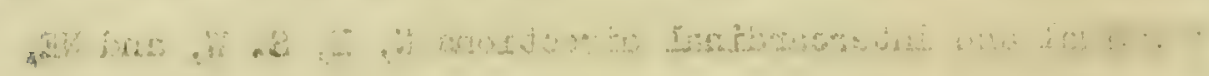

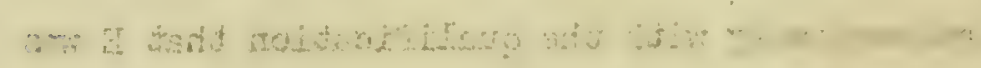

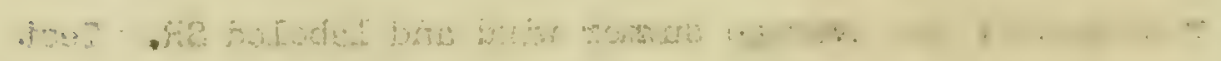

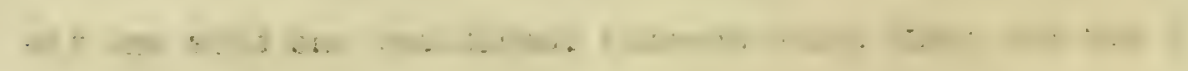

$\therefore \quad \therefore \quad+\therefore \quad \therefore \quad \div \quad \div \quad \div$

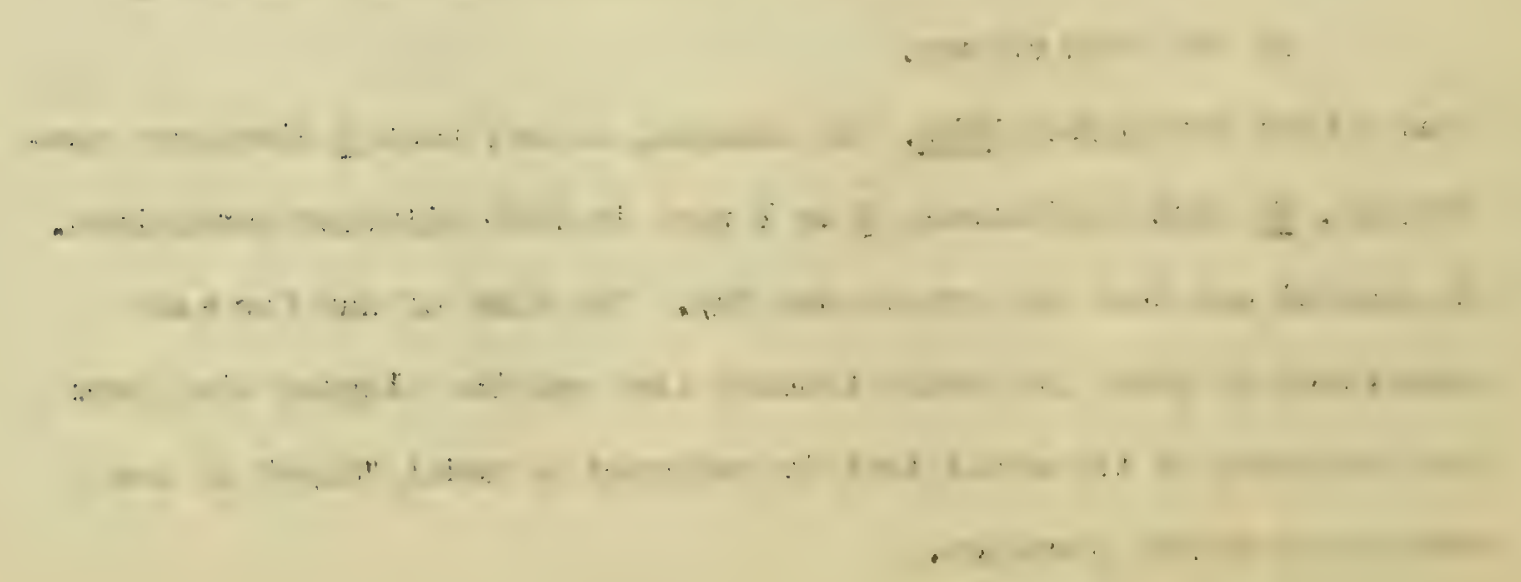




\section{October 1949 PRELIMTNARY TESTS OF EQUIPMENT}

\section{TRIAL OF FRESH WATER (RAINFALL) DISCHARGE EXPERTMENT}

Model filled to tide datum with clear fresh $(p=1.000)$ tap water. Brom Cresol Green solution added at Littio Lake equivalent box to trace fresh water drainage pattern.

Tide machine set to run at $31.5 \mathrm{sec}$. semi-diurnal cycle. Fresh water discharge rate adjusted to $168 \mathrm{cc} / \mathrm{min}$.

Density difference zero, in this case.

Cameras used for all model rums; (at a working distance

16 feet over center of model except 3 at $\boldsymbol{H}_{4} \mathrm{ft}$.).

1. Graflex Photorecord pneumatic camera, $35 \mathrm{~mm}$ double frame, Bolsey f-3.2 Wollensak Anastigmat, $44 \mathrm{~mm}$ f.l.; Bolsey shutter, automatic exposure interval $7.5 \mathrm{sec} .$, Exp. 1/10 sec., f-9.5, 800 exp./roll

2. Paillard Bolex time-lapse motion-picture camera, $16 \mathrm{~mm}$ single frame, Eastman Kodak I-2.7 Cine Anastigmat, $15 \mathrm{~mm}$ f.I., automatic exposure interval I second, $1 / 30 \mathrm{sec}$, f-6.3, $95 \mathrm{ft}$. rolls, $3800 \mathrm{exp} . / \mathrm{roll}$

3. Leica C-III, $35 \mathrm{~mm}$ double frame hand camera, Wollensak f -4.5 Velostignat f.1. $127 \mathrm{~mm}$, manual exposure control, exp. I/8 sec., f-1I, working distance 14 feet over F.S. Co. operating area

Film Kodachrome Iype $A$ in 100 foot $35 \mathrm{~mm}$ spools for Graflex (1)

Kodachrome Type A in 95 foot $16 \mathrm{~mm}$ spools for Bolex (2) cut down from 200 foot spools

Kodachrome Type $A$ in 20 and 36 exposure cassettes for Leica (3)

Illumination 12 No. 2 Photoflood lamps in reflectors operating at 135 volts, A.C.

30 ot. 149

1405

1419

1420

1427

1428

1516

1518

1550

1600

1619

1626

1643

1658

1736

Brom Cresol Green added to equivalent box, cameras started, no wind, clock omo oda $0^{\text {hr }}$

Brom Cresol Green added to equivalent box

Cameras jammed

Cameras cleared

Cameras jammed, trouble in gravity trigger, secured run. Drained model, refilled with fresh tap water to $\mathrm{msl}$ datum, camera triggers repaired, test repeated

Cameras restarted

Brom Cresol Green addod to equivalent box

" " " " " " " "

Reloaded $16 \mathrm{~mm}$ camera.

$16 \mathrm{~mm}$ take-up spool jammed for approxinately $30 \mathrm{sec}$. before bcing cleared

$35 \mathrm{~mm}$ magazine emptied

Brom Cresol Green added to equivalent bax

Test sccured. At 105 "days" fresh water discharge reached

Lake Washington 


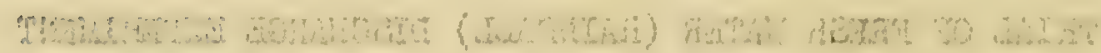

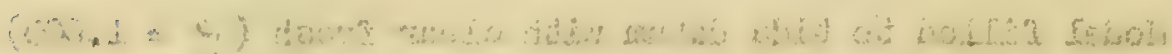

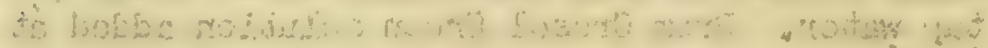

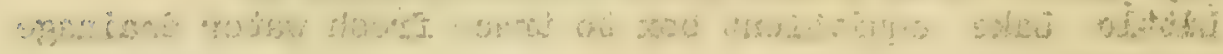

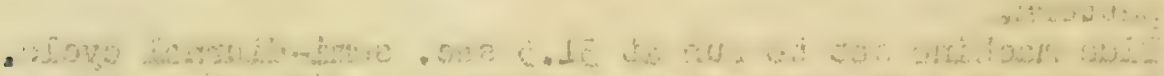

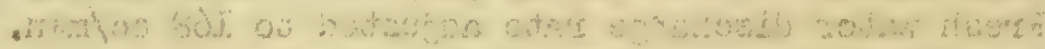

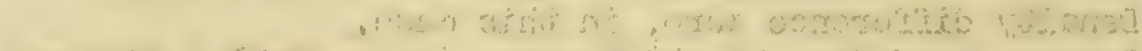

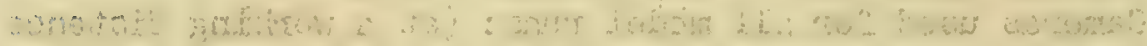

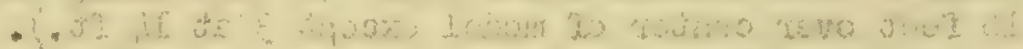

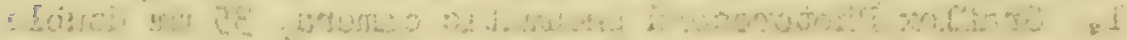
- $\begin{gathered}\text { 2. } \\ \text { a }\end{gathered}$

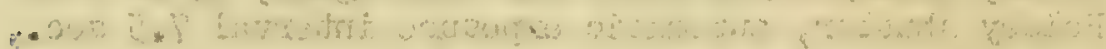

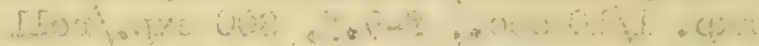

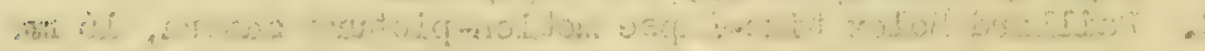

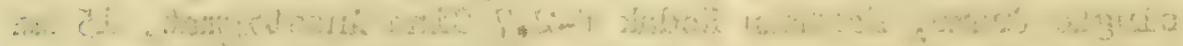

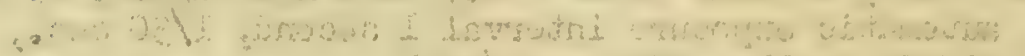

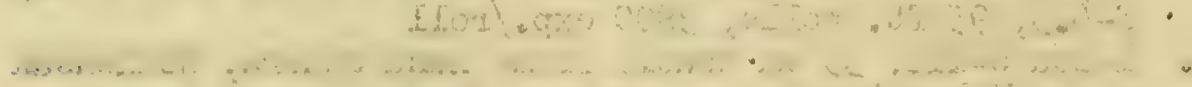

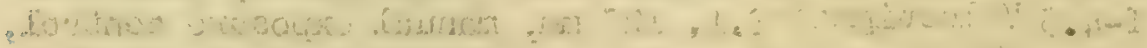

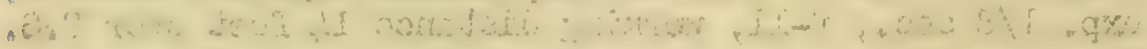
and a d a

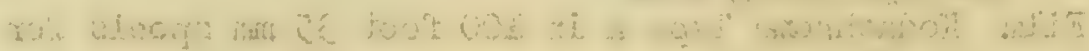

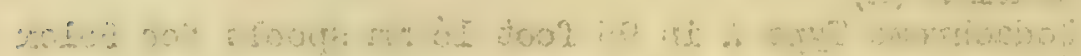

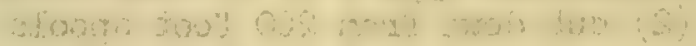

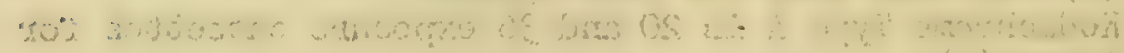

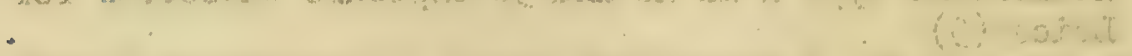

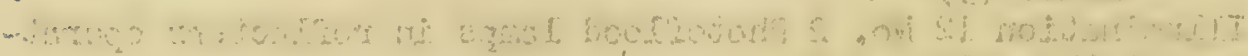

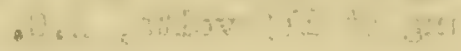

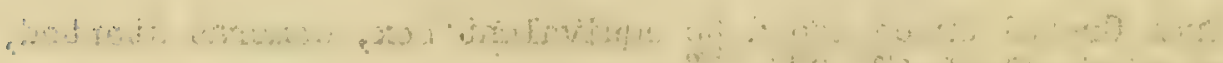

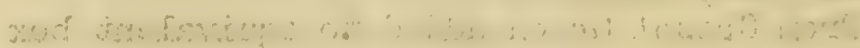


FREEPORT SULPHUR CO. BARATARIA BAY MODEI TESTS

4 october 1949 ACID AND WATER DENSITY* ADJUSTMENTS

Two 4-liter Ehrlenmeyer flasks prepared with 3-4 liters of Dil. $\mathrm{H}_{2} \mathrm{SO}_{4}$ as follows: 1. Labeled "200:I" contalning approximately $0.1 \mathrm{~N} \mathrm{H}_{2} \mathrm{SO}_{4}+\mathrm{Brom}$

2. Labeled "1000: I" containing approximately $0.5 \mathrm{~N} \mathrm{H}_{2} \mathrm{SO}_{4}+\mathrm{Brom}$ Cresol Green sp.gr, $=1.033$ at $60^{\circ} \mathrm{F}$

$\begin{array}{llr}\text { average sp.gr. of prototype Point VI } & 1.057 \\ \text { bieed water } & \text { Point VIII } & 1.024 \\ \text { at } 600 \mathrm{~F} & \text { raw } & \frac{1.055}{3.136} \\ & & \frac{32.045}{1.045}\end{array}$

average sp.gr. of amblent water at $60^{\circ} \mathrm{F} \quad \frac{1.012}{0.033}$

Difference

$1+\Delta \rho \equiv 5 \%$ NaCl solution $\rho=1.033 \mathrm{gm} / \mathrm{cc}$.

Sp.gr. of " $1000: I^{\prime \prime}$ acia solution at $60^{\circ} \mathrm{F}=1.033$ without

adjustment but "200:1" sp.gr. was 1.006. Increased specific gravity of "200:1" solution by adding $\mathrm{Na}_{2} \mathrm{SO}_{4}$ crystals until hydrometer read sp.gr. $=1.033$ at $600 \mathrm{~F}$. Equivalent $\Delta ?$ for both solutions $0.033 \mathrm{gm} / \mathrm{cc}$. greater than ambient water of density $1.000 \mathrm{gm} / \mathrm{cc}$.

Additional solutions made up for titration control during tests. 1. Intermediate standard $\mathrm{H}_{2} \mathrm{SO}_{4}$ solution approximately $0.01 \mathrm{~N}$, $=" 200: I "$ solution cut nominally $10: I$.

2. Reference $\mathrm{Na}_{2} \mathrm{CO}_{3}$ solution approx. $0.2 \mathrm{~N}$ for comparison of acid solutions?

Titration of "200:I" solution and 1/10 cut "200:1" solution showed actual cut to be $9.94: 1$

Titration of "I000:I" solution and $1 / 50$ cut "I000:I" solution showed actual cut to be $50: 2: 1$

Thus dilution factor nominally "200:1" is

$9.94 \times \frac{\text { (50 cc volume sample of ambient Gulf water) }}{\text { (volume intermediate standard acid to neutralize }}$ in fact,

and dilution factor nominally "1000: 1 " is

(50 cc volume sample of ambient Gulf water)

$50.2 \times$ (volume intermediate standard acid to neutralize ${ }_{\text {Gulf sample) }}$ in fact.

* Density is ordinarily considered in model theory but in practice specific gravity is the quantity most convenientiy measured. Therefore, density in each case was measured in terms of specific gravity at $60^{\circ} \mathrm{F}$. The results are numericaliy equivalent to Gensity but differ dimensionaliy as indicated. 

Isolated discharge from points VI and VIII at F.S. Co. mining area for 1946. BOM

fresh water discharge set Sample ml.stand.acid at $168 \mathrm{cc} / \mathrm{min}$. $450 \mathrm{x}$ $10^{6} \mathrm{ft}, 3 / \mathrm{day}$

bleed water discharge equivalent to $2.4 \times 10^{6}$ gal./day

1245 $0 \mathrm{da}$ bleed water discharge started

$1245^{\circ} 0$ da start $35 \mathrm{~mm}$ at 0 exp. $1 / 10 \mathrm{sec} . \mathrm{f}-9.5$

12450 da start $16 \mathrm{~mm}$ at $0 \mathrm{ft}$. $f-6.3$

1300 $\mid$ Secured. Shutter main spring fouled. Stopped run at 150 exp. Flushed model

BOM

14230 da

bleed water discharge started

1423: 0 da $\mid \begin{aligned} & \text { start } 35 \mathrm{~mm} \text { at } 150 \text { exp. } \\ & 1 / 10 \text { sec. }-9.0\end{aligned}$

1423 0 da start $16 \mathrm{~mm}$ at $25 \mathrm{ft}$. $1-6.3$

$143914 \mathrm{da}$ exp. 4. Leica $127 \mathrm{~mm}$ $1 / 8$ sec. $\{-11$

$145328 \mathrm{da}$ exp. 5. Leica $127 \mathrm{~mm}$ $1 / 8$ sec. $f-11$

1505 Note: bleed water "stores up" during diurnal tides and "expands" on tropical

150742 da exp, 6 . Leica $127 \mathrm{~mm} 1 / 8$ sec. $1-11$

151650 da changed $16 \mathrm{~mm}$ spools

152156 da exp.7. Leica $1 / 8 \mathrm{sec}$. $[-1]$

1525:60 da exp. 8. Leica $1 / 8 \mathrm{sec}$. If-11

$1526 \quad 61$ da $16 \mathrm{~mm}$ camora missing; adjusted trigger stroke

154681 da exp.9. Leica $1 / 8 \mathrm{sec}$. f-11

154883 da changed $35 \mathrm{~mm}$ magazines 1600: $95 \mathrm{da}$ exp. 10. Leica $1 / 8 \mathrm{sec}$. [-1]

1615,110 da $35 \mathrm{~mm}$ magazine balky; moved film to old magazine

\section{time} initial final

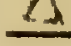
end point

17.75

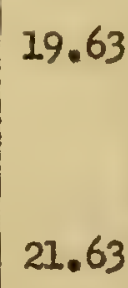

1.88

bl.gr.

1511

19.63

2.00

bl.gr. 


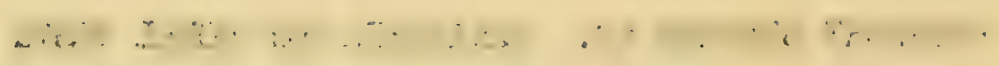

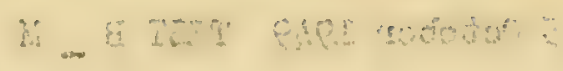

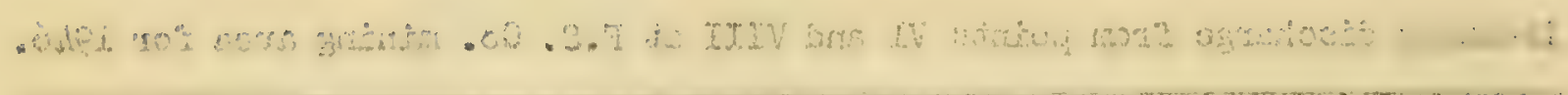

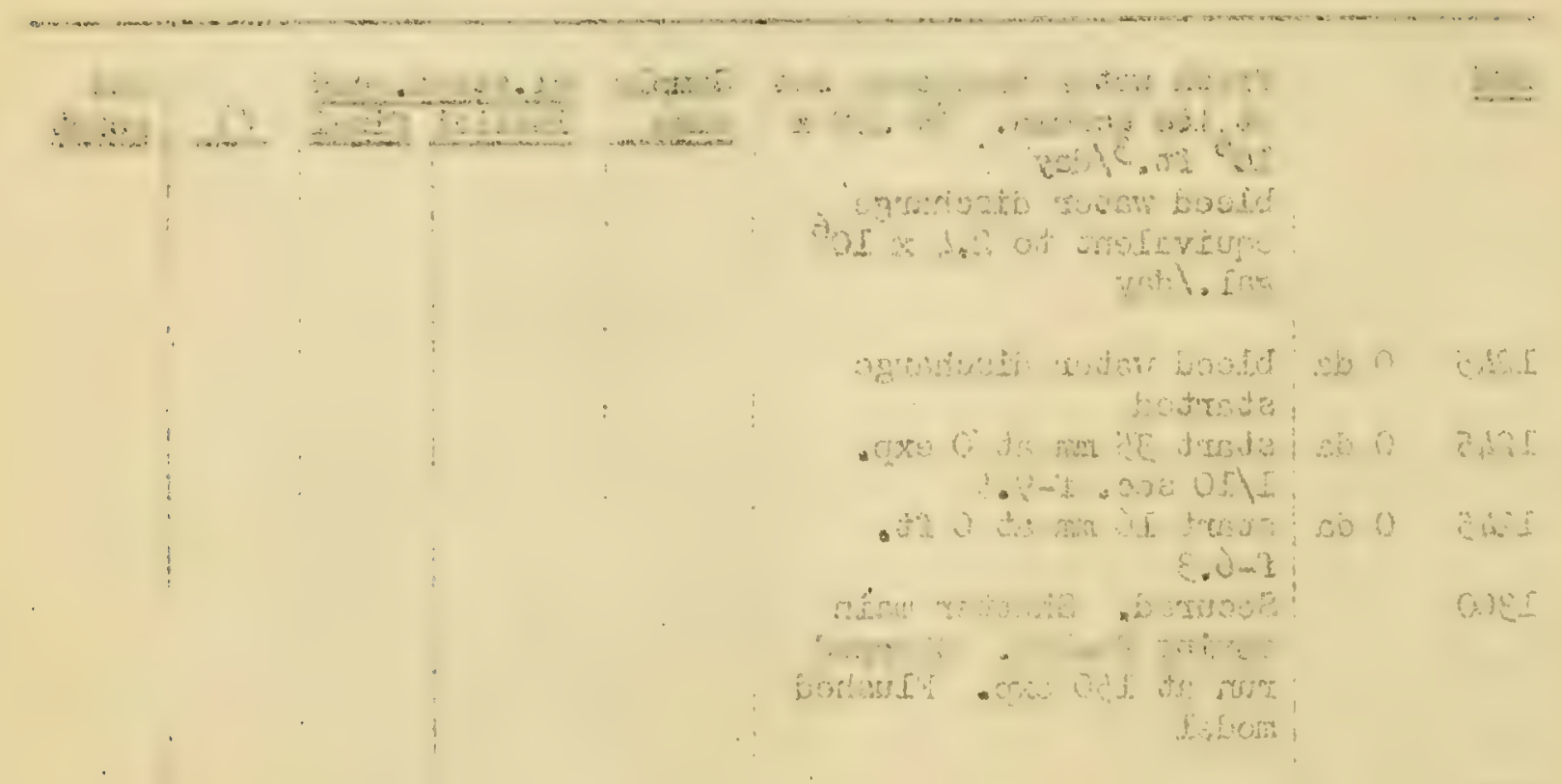

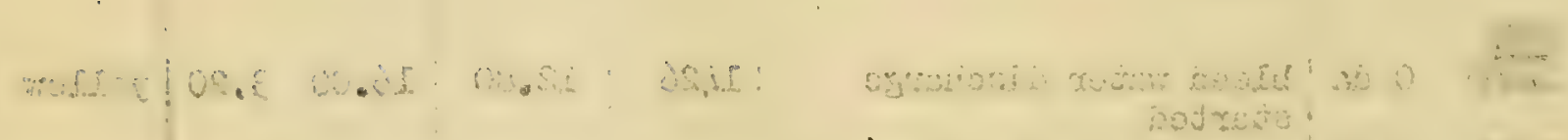

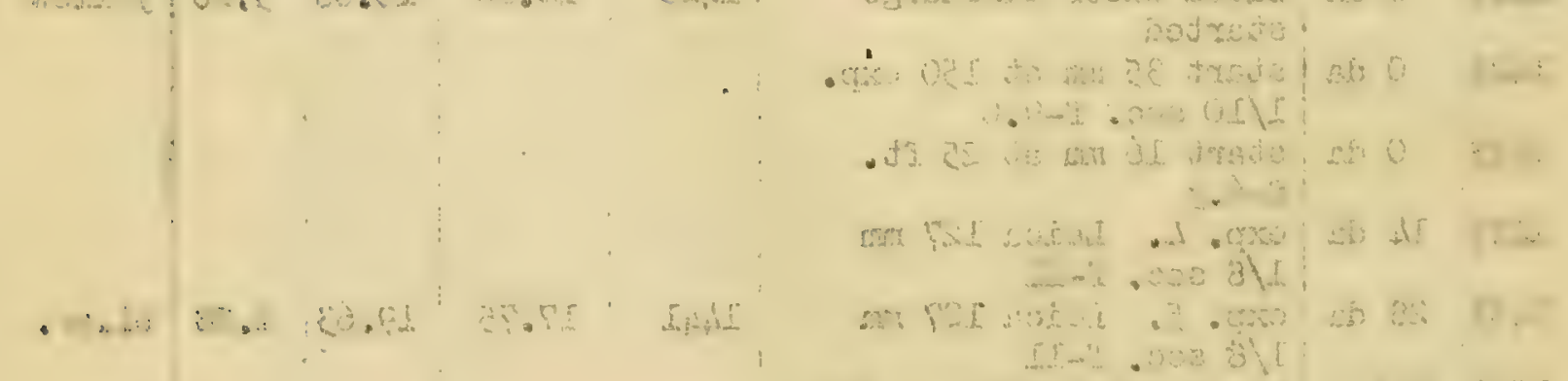

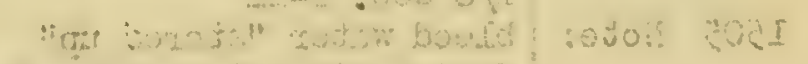

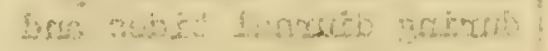

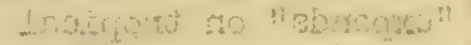

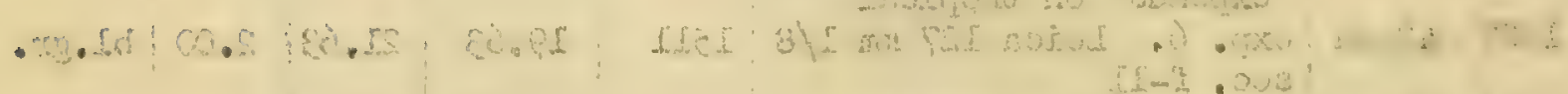

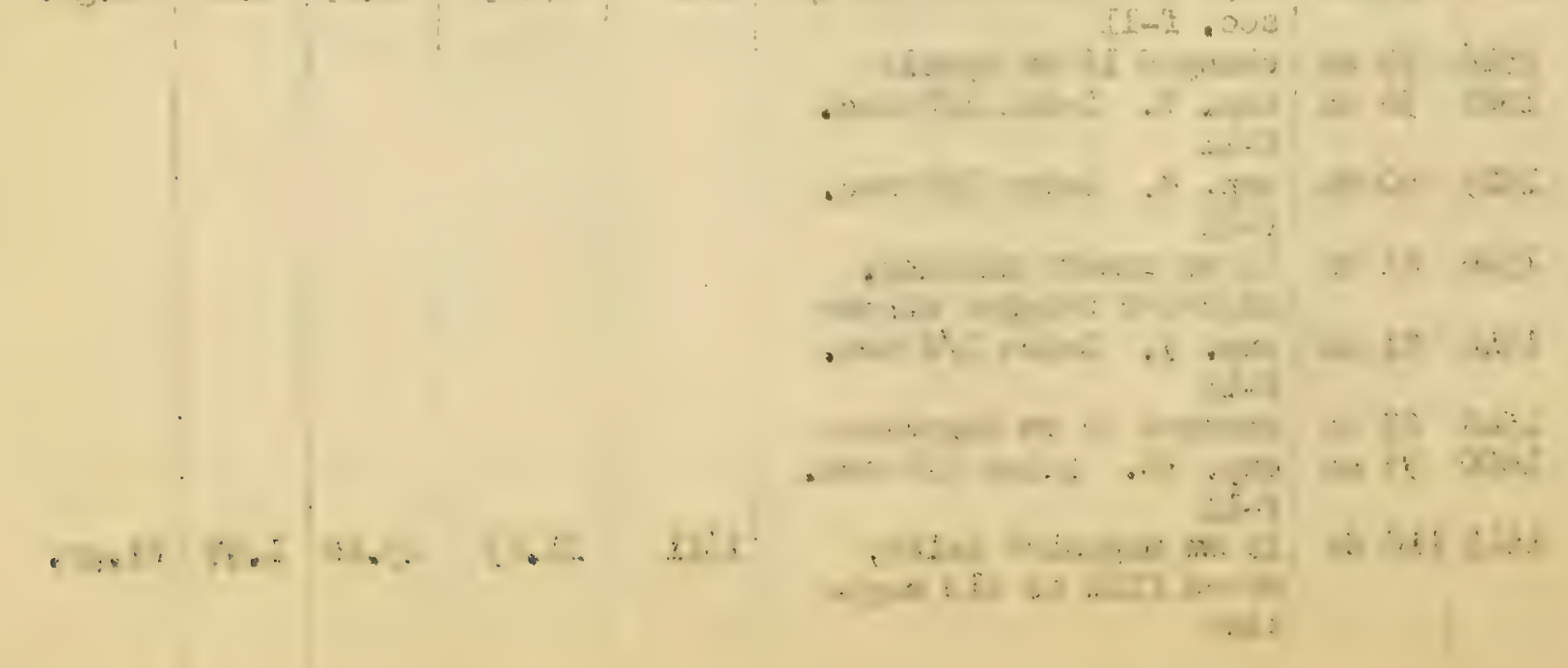




\begin{tabular}{|c|c|c|}
\hline BOM & & \\
\hline 1625 & $120 \mathrm{da}$ & $\exp _{f-11}$ 11. Leica $1 / 8 \mathrm{sec}$ \\
\hline 1635 & $120 \mathrm{da}$ & $\begin{array}{l}16 \mathrm{~mm} \text { camera film } \\
\text { changed }\end{array}$ \\
\hline 1647 & $142 \mathrm{da}$ & $\exp _{f-11}$ 12, Leica $1 / 8 \mathrm{sec}$ \\
\hline 1659 & $155 \mathrm{da}$ & $\exp _{f-11} 13$. Leica $1 / 8 \mathrm{sec}$ \\
\hline 1725 & $180 \mathrm{da}$ & $\exp _{f-11}$ 14. Leica $1 / 8 \mathrm{sec}$ \\
\hline $\begin{array}{l}1725 \\
1725\end{array}$ & $\begin{array}{l}180 \mathrm{da} \\
180 \mathrm{da}\end{array}$ & $\begin{array}{c}\text { secured; } 35 \mathrm{~mm} 750 \mathrm{exp} . \\
\| . \quad 16 . \mathrm{mm} .95 \mathrm{ft} .\end{array}$ \\
\hline
\end{tabular}

Note: Last seven days to be ignored - 50 drops of bleed water $\left(50 \times 10^{6}\right.$ gal. approximately) accidentally discharged 10 "days" before SR wind experiment began. Bay Long heavily colored during lst week of SR run but returned to normal during second week.

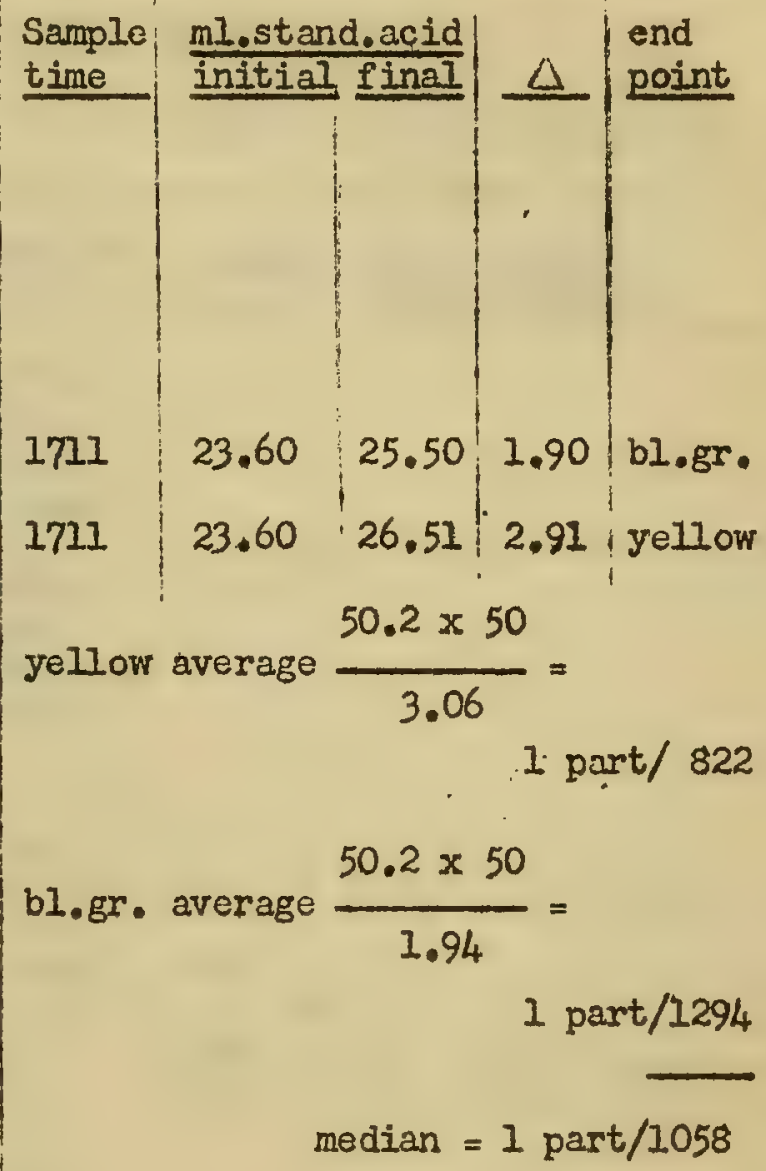

\begin{tabular}{l|l|l} 
BSRM & \\
1729 & da & SR wind started
\end{tabular}

1739 $10 \mathrm{da}$ exp. 15. Leica $1 / 8 \mathrm{f}-11$

$174618 \mathrm{da}$ started $16 \mathrm{~mm}$ camera $0 \mathrm{ft}$.

$175426 \mathrm{da}$ " $35 \mathrm{~mm}$ camera

1758, $30 \mathrm{da}$ exp. 16. Leica 1/8 f-11

1808 $40 \mathrm{da}$

181742 da

$181142 \mathrm{da}$

"17. " " "

"18. " $1 "$

stopped $35 \mathrm{~mm}$ camera at 150 exp.

$181142 \mathrm{da}$ stopped $16 \mathrm{~m}$ camera at $39 \mathrm{ft}$.

fresh water discharge checked at $170 \mathrm{cc} / \mathrm{min}$. 


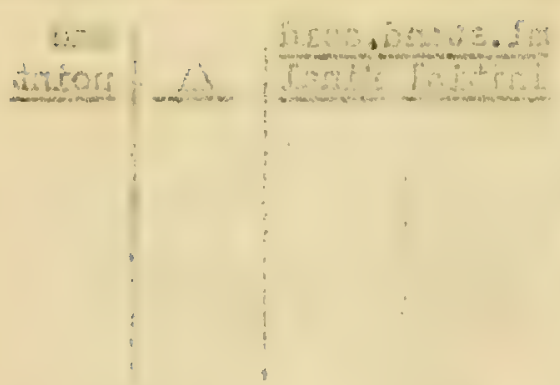

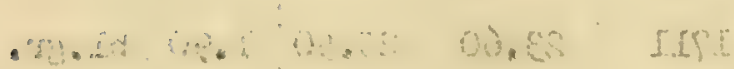

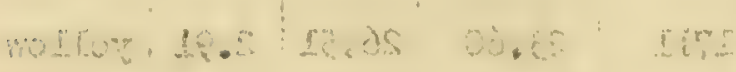

$$
=\frac{\partial}{x+3}
$$

$5.2+3+2+2$

$$
\frac{2 x-6}{40.5}
$$

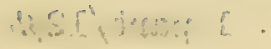

a......

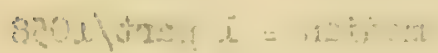

Soura:

2

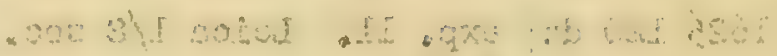
$51-\infty$

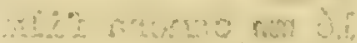
x.mensisto

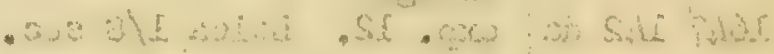

Lix $\therefore$ OI

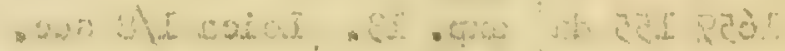

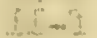

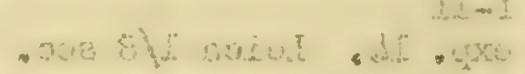
(1) net $=5 x$ $55^{2}-2$

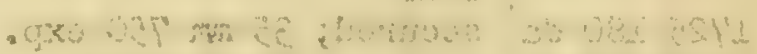

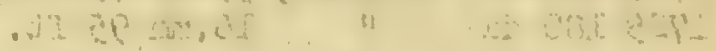

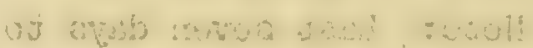

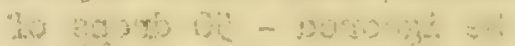

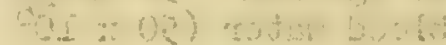

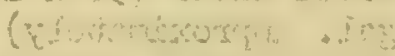

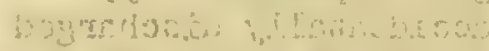

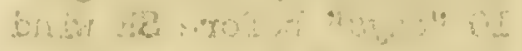
r.

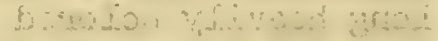

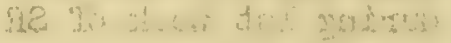

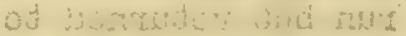

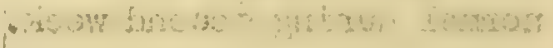

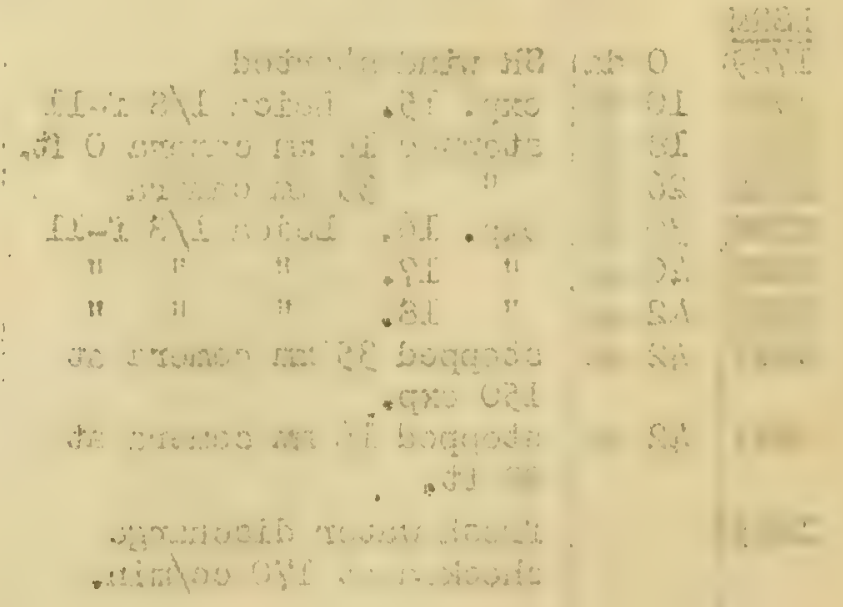


FREEPORT SULPHUR CO. BARATARIA BAY MODET TESTS

$$
6 \text { October } 1949 \text { TEST B _ M }
$$

Isolated discharge from paints VI and VIII at F.S. Co. mining area for 1946.

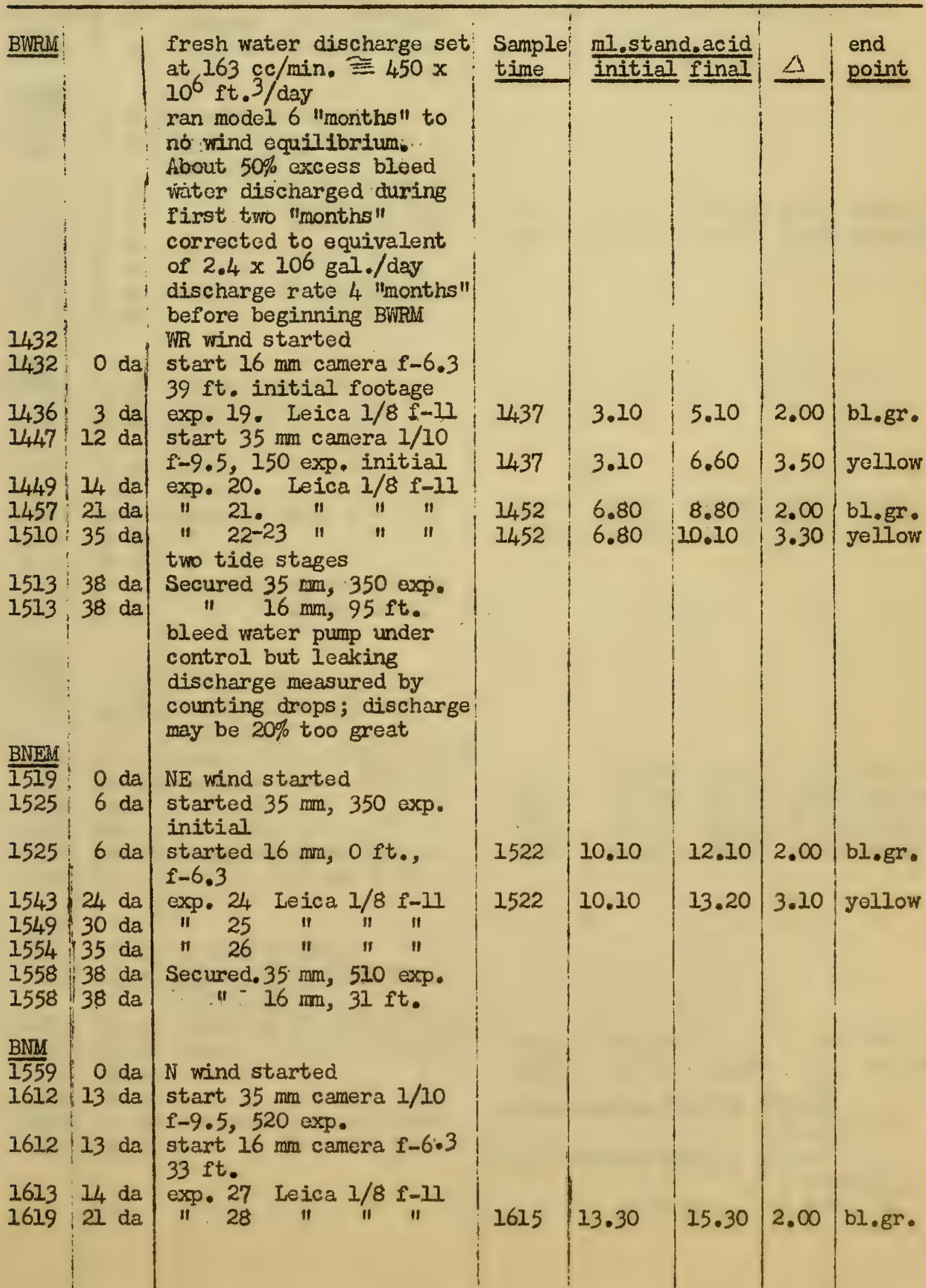




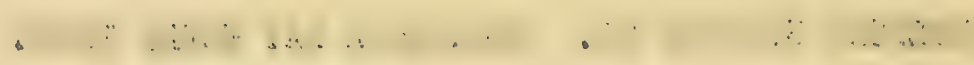

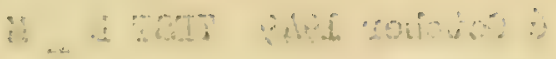

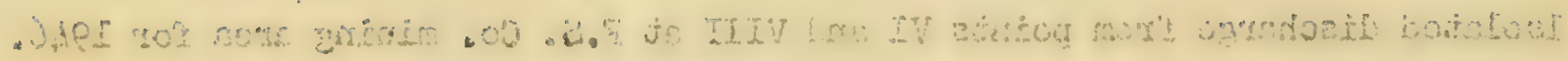

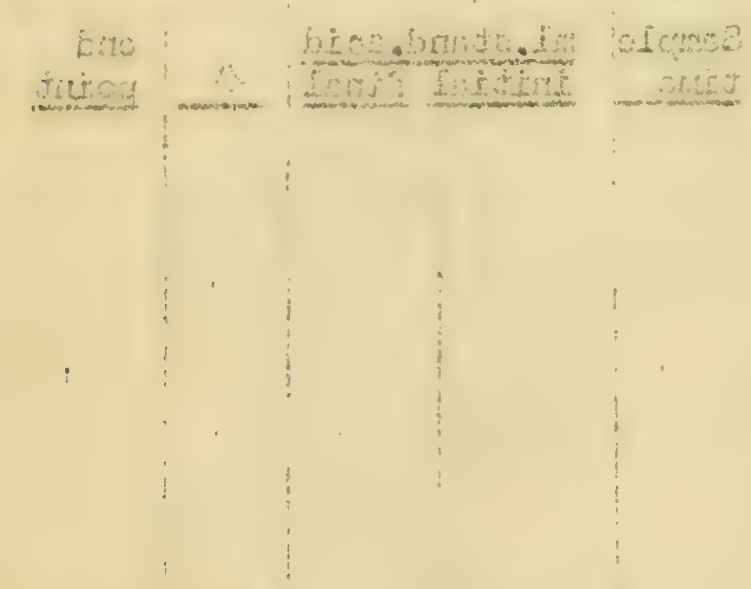

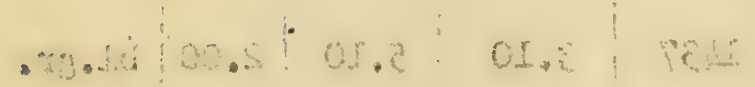
खLiar

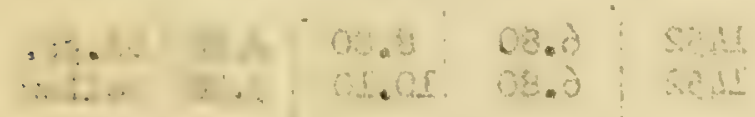

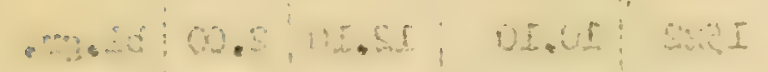

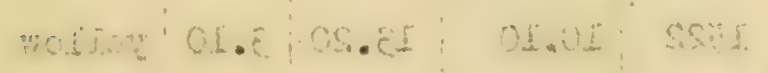

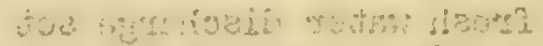

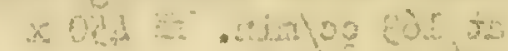

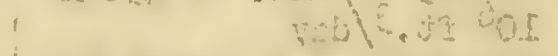

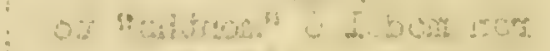

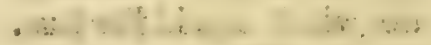

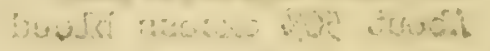

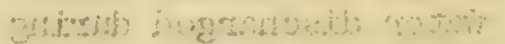
;

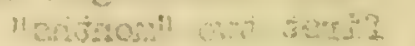

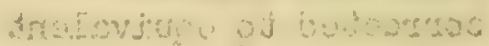

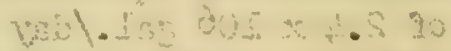

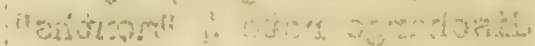

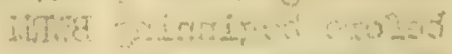

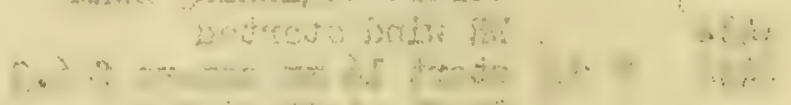

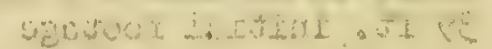

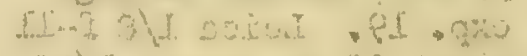

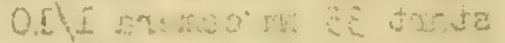
trotude -

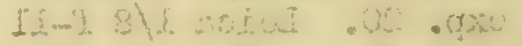

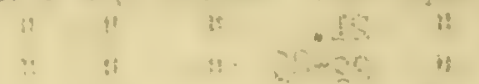
-

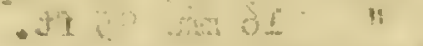

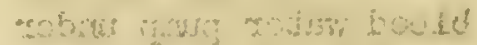

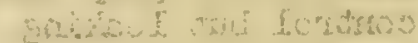

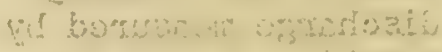

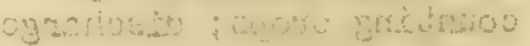

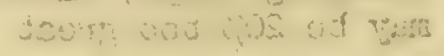

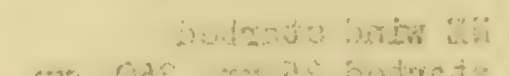

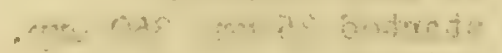
Lisitire. $\therefore \quad \because \therefore$ $\therefore \quad \therefore$

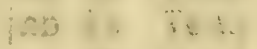
$\therefore \therefore$

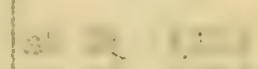
$\therefore \quad \therefore \quad \therefore$

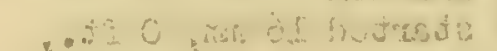
ह. तो-? $15-2$ is 50 .

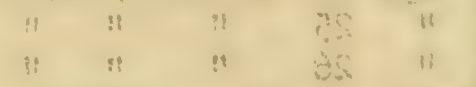

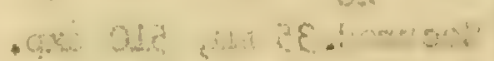

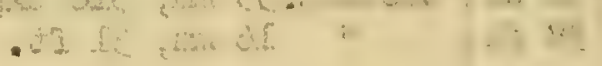
$\ldots$ $\because 8$ $\therefore$ $\cdots$

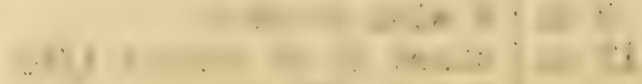
$\therefore+\cdots+\cdots$ : : : $\quad$ : 1. . . . . : 
BNM

1627 28 da exp. 29 Leica $1 / 8$ f-11 $163030 \mathrm{da}$ secured; $35 \mathrm{~mm} 660$ exp. 1630 ) $30 \mathrm{da}$ " $16 \mathrm{~mm} 58 \mathrm{ft}$.

BNWM

1631 O da NW wind started

$1644 \cdot 13$ da start $35 \mathrm{~mm} \mathrm{1/10} \mathrm{f-9.5}$ 660 exp.

$164413 \mathrm{da}$ start $16 \mathrm{~mm} \mathrm{f}-6.358 \mathrm{ft}$. 164514 da exp. 30 Leica $1 / 8 \quad\{-11$ $165625 \mathrm{da}$ " 31 " " 1659 28 da " 32 " " " 170331 da secured; $35 \mathrm{~mm} 800$ exp. 1703 31 da! " $16 \mathrm{~mm} 87 \mathrm{ft}$.

BWM 1704 da wind started

171713 da start $35 \mathrm{~mm} 0$ exp.

$171713 \mathrm{da} / \begin{aligned} & 1 / 10 \mathrm{f}-9.5 \\ & \text { start } 16 \mathrm{~mm} 0 \mathrm{ft} .\end{aligned}$

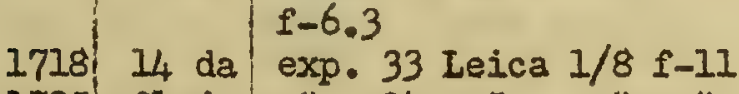

1725,21 da

$173228 \mathrm{da}$

1735,31 da

1735

31 da

BSWM:

1737

1750 ' 13 da

$175013 \mathrm{da}$

$\begin{array}{llll}1751 & 14 & d a\end{array}$

1758 21 da

$180528 \mathrm{da}$

$180831 \mathrm{da}$

1808

BSM

1809

$u_{4}$ da start $35 \mathrm{~mm} 310$ exp.

$1 / 10 \mathrm{f}-9.5$

$18231_{4}$ da start $16 \mathrm{~mm} 58 \mathrm{ft} . \mathrm{f}-6.3$ 1823. 14 da $\operatorname{exp.~I~Leica~} 1 / 8 \quad f-1]$

1830

1838

1841

1841
31 da

SW wind started

$1 / 10$ f -9.5

start $16 \mathrm{~mm} 31 \mathrm{ft} . \quad 1-6.3$

exp. 36 Leica $1 / 8$ f-11

" 37 " " "

secured; 35 mm 310 exp.

" $\quad 16 \mathrm{~mm} 58 \mathrm{ft}$.

21 da

i" 2

$28 \mathrm{da}$

31 da 11

secured $35 \mathrm{~mm} 450$ exp. 31 da !n $16 \mathrm{~mm} 85 \mathrm{ft}$.

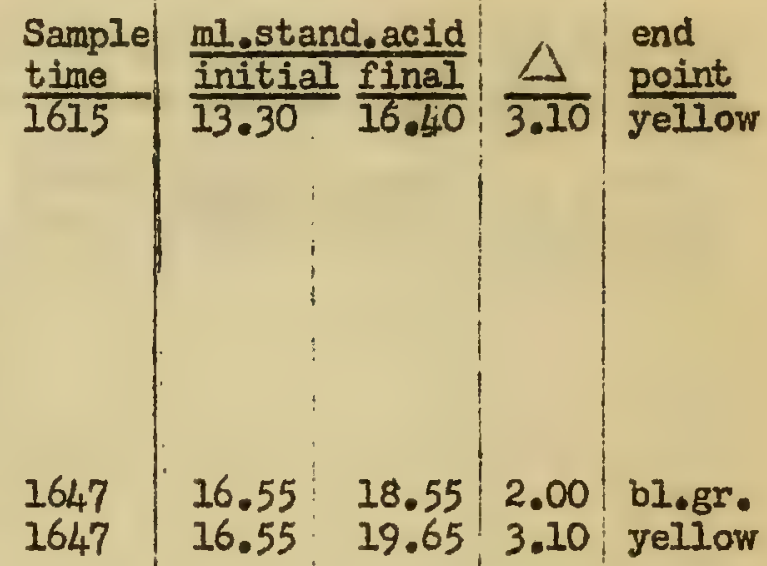

1755 22.90? 25.30 2.403 bl.gr.

\begin{tabular}{l|l|l|l|l}
1755 & $22.90 ?$ & 26.10 & 3.20 & yellow
\end{tabular}

\begin{tabular}{l|l|l|l|l}
1758 & 26.10 & 28.10 & 2.00 & $\mathrm{bl} . \mathrm{gr}$.
\end{tabular}

1758 26.10, $29.10 \quad 3.00$ yellow

1825

1825

\begin{tabular}{ll|l|l|l}
29.30 & 31.30 & 2.00 & bl.gr.
\end{tabular} $29.30,32.40 \quad 3.10$ yellow yellow average $150.2 \times 50 / 3.16=$ b1.gr. average $(50.2 \times 501 / 2,00=$ 793 
FREEPORT SULPHUR CO. BARATARIA BAY MODEL TESTS

7 October TEST B - CC

Isolated discharge from points VI and VIII at F.S. Co, mining area for 1946.

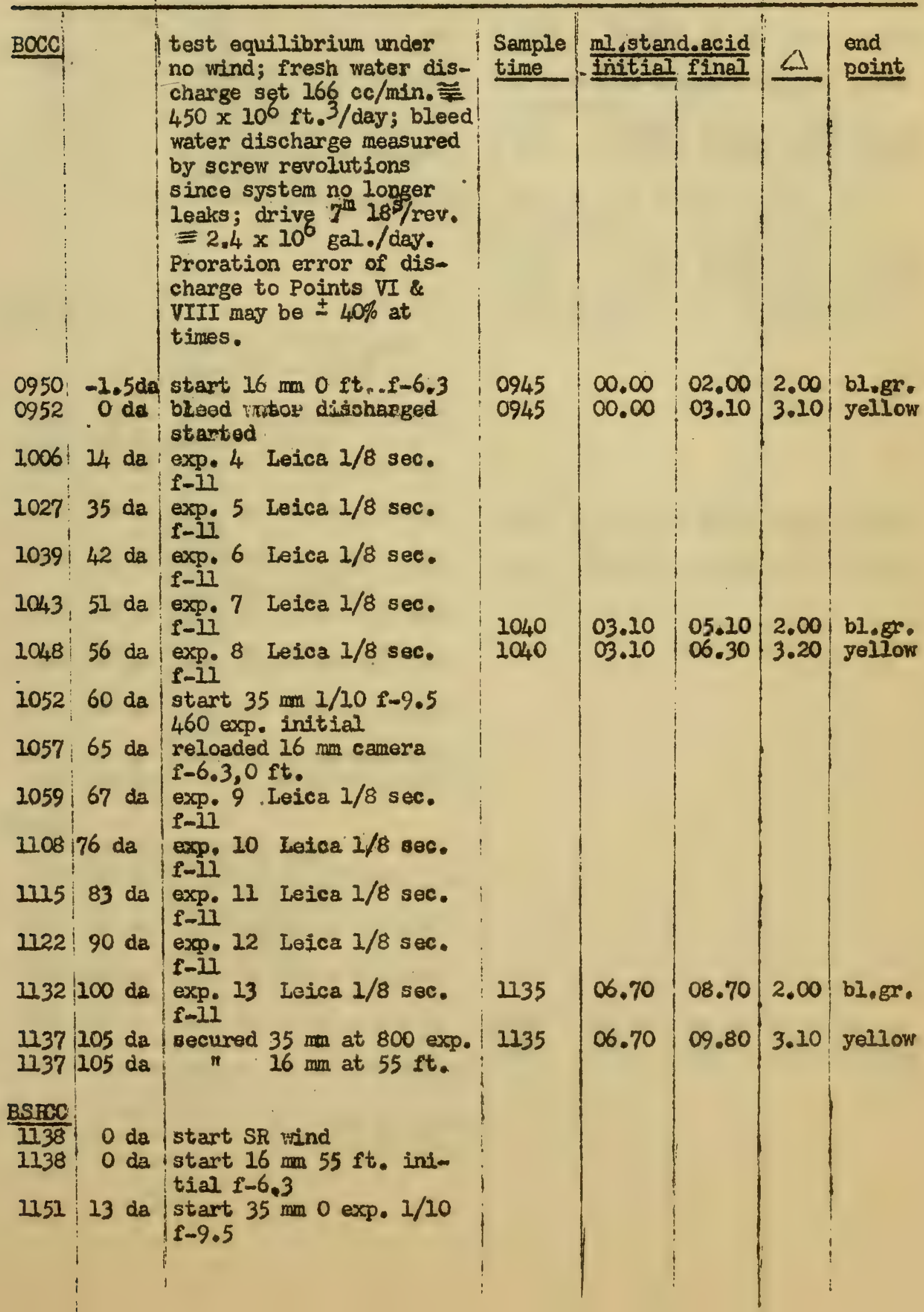




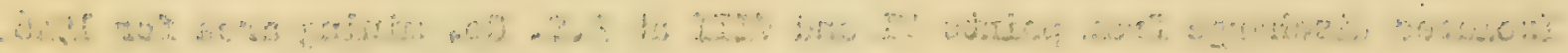

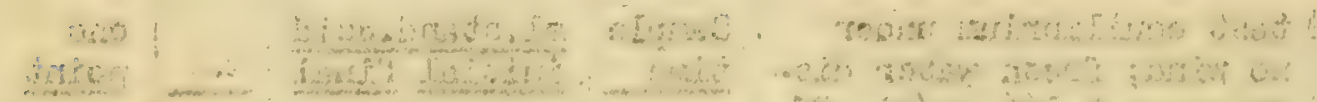

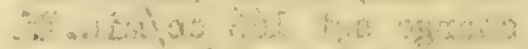

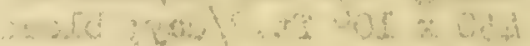

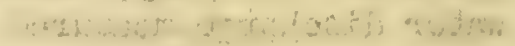

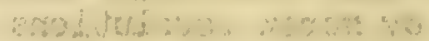

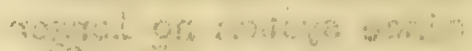

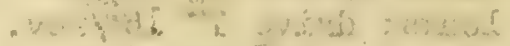

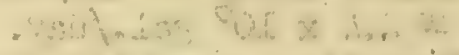

$$
\begin{aligned}
& \text { 2) } 18 \text { a } \\
& \text { L }
\end{aligned}
$$

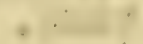

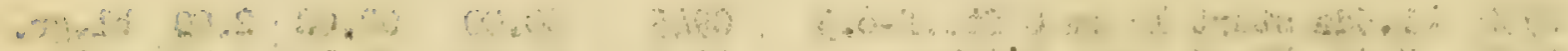

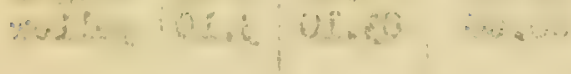

a

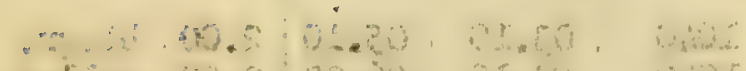

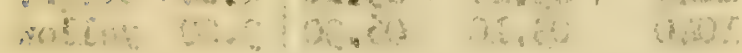

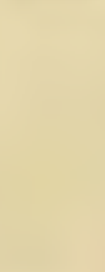




\begin{tabular}{|c|c|c|}
\hline BSRCC & & \\
\hline 1152 & $I_{4} \mathrm{da}$ & $\exp _{1 \text { ii }}$ I $_{4}$ Ietca $1 / 8 \mathrm{sec}$ \\
\hline 1159 & $22 \mathrm{da}$ & $\exp _{\text {I-il }} 15$ Ieica $1 / 8$ sec. \\
\hline 1206 & & 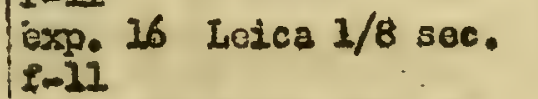 \\
\hline $1207 \%$ & $\begin{array}{l}29 \mathrm{da} ? \\
30 \mathrm{da}\end{array}$ & $\begin{array}{l}\text { 16. Iiln ran out, no } \\
\text { time to reload before } \\
\text { end of run } \\
\text { secured } 35 \mathrm{~mm} \text { at } 245 \text { exp. }\end{array}$ \\
\hline
\end{tabular}

BWRCC

retumed to no wind equilibrium 1208 to 1312

1312 - 1 da start $16 \mathrm{~mm} 5-6.30 \mathrm{ft}$

1313 O da start WR wind

13130 da exp. 17 Leica $1 / 8$ soc. I- II

13207 da $\exp _{1} 18$ Leica $1 / 8 \mathrm{sec}$.

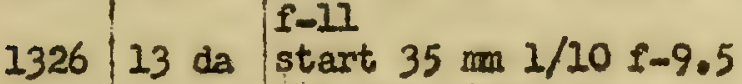

145 exp. initial

$1327 / 14$ da expi 19 Leica $1 / 8 \mathrm{sec}$ f-11

133421 da exp. 20 Laica I/8 sec. |f-1]

1341 $28 \mathrm{da}$ exp, 21 Leica $1 / 8 \mathrm{sec}$. $f-i$

1344 31 da secured $35 \mathrm{~mm}$ at 295 exp.

1344 31 da " $16 \mathrm{~mm}$ at $49 \mathrm{ft}$.

BNECC

1345

0 da

start NE wind

$1 3 5 8 \longdiv { 1 3 } \mathrm { da }$

start 35 am 295 exp. initial $1 / 10 \quad I-9.5$

$135813 \mathrm{da}$ start $16 \mathrm{~mm} 49 \mathrm{ft}$. InItial $f-6.3$

135914 da exp. 22 Leica $1 / 8 \mathrm{sec}$. I-1]

$1406 / 21$ da exp. 23 Leica $1 / 8$ sec. f-11.

141328 da exp. 24 Ielca $1 / 8 \mathrm{sec}$. f- 11

1416 31 da secured $35 \mathrm{~mm}$ at 440 exp. $1416: 31 \mathrm{da}$ 1" $16 \mathrm{~mm}$ at $75 \mathrm{ft}$. Sample ml,stand,acid $\Delta$ end $1155, \overline{09.85}, \frac{12.10}{2.35}, \overline{b 1.85}$. \begin{tabular}{ll|l|l|l}
1155 & 09.85 & 13.20 & 3.35 & yellow
\end{tabular} Brom Cresol Green $+\mathrm{Na}_{2} \mathrm{CO}_{3}$ added at venturi $1200 \quad 13.20|15.40| 2.20$ bl.gx. $1200: 13.20 \quad 16.40 \quad 3.20$ yellow Brom Cresol Green $+\mathrm{Na}_{2} \mathrm{CO}_{3}$ added at renturi \begin{tabular}{l|l|l|l|l|l|l|l|} 
& & \\
1205 & 16.40 & 18.60 & 2.20 & $\mathrm{bl} . \mathrm{gr}$.
\end{tabular} $1205 \quad 16.40 \quad 19.80 \mid 3.40$ yellow Brom Cresol Green $+\mathrm{Na}_{2} \mathrm{CO}_{3}$ added at venturi

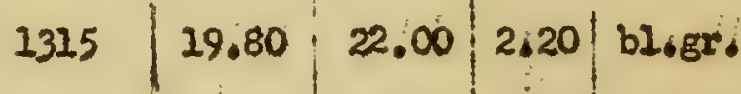 \begin{tabular}{ll|l|l|l}
1315 & 19.80 & 23.10 & 3.30 & yellow
\end{tabular}}

408 1408 


\begin{tabular}{|c|c|c|c|c|c|c|c|}
\hline BNCC! & & & $\begin{array}{l}\text { Sample } \\
\text { time }\end{array}$ & $\frac{\text { ml.sta }}{\text { initia }}$ & $\frac{\text { d. acid }}{\text { final }}$ & $\Delta$ & $\begin{array}{l}\text { end } \\
\text { point }\end{array}$ \\
\hline 14.17 & $0 \mathrm{da}$ ! & $\begin{array}{l}\text { start } N \text { wind (wind } \\
\text { indicator unstable) }\end{array}$ & & & & & \\
\hline 1430 & $13 \mathrm{da}$ & $\begin{array}{l}\text { start } 35 \mathrm{~mm} \text { at } 440 \text { exp. } \\
1 / 10 \mathrm{f}-9.5\end{array}$ & & & & & \\
\hline 1430 & $13 \mathrm{da}$ & start 16 m 0 ft. $f-6.3$ & $\begin{array}{l}1432 \\
1432\end{array}$ & $\begin{array}{l}17.80 \\
17.80\end{array}$ & $\begin{array}{l}19.90 \\
21.10\end{array}$ & $\begin{array}{l}2.10 \\
3.30\end{array}$ & $\begin{array}{l}\text { bl.gr. } \\
\text { yellow }\end{array}$ \\
\hline 1436 & $18 \mathrm{da}$ & $\exp _{f-1 i} 25$ Lejea $1 / 8$ sec. & & & & & \\
\hline 1438 & $21 \mathrm{da}$ & $\exp _{f-11} 26$ Leica $1 / 8 \mathrm{sec}$ & & & & & \\
\hline 1445 & $28 \mathrm{da}$ & $\exp _{f-11} 27$ Leica $1 / 8 \mathrm{sec}$ & & & & & \\
\hline $\begin{array}{l}1448 \\
1448\end{array}$ & $\begin{array}{l}31 \mathrm{da} \\
31 \mathrm{da}\end{array}$ & $\begin{array}{c}\text { securad } 35 \mathrm{~mm} \text { at } 590 \text { exp.! } \\
\text { " } 16 \mathrm{~mm} \text { at } 26 \mathrm{ft} .\end{array}$ & & & & & \\
\hline & & $\begin{array}{l}\text { stabilized wind sack by } \\
\text { oil damping }\end{array}$ & & & & & \\
\hline$\frac{\text { BNwCC }}{1449}$ & 0 da & start NWF wind & & & & & \\
\hline 1503 & $14 \mathrm{da}$ & $\begin{array}{l}\text { start } 35 \mathrm{~mm} \text { at } 590 \text { exp. } \\
1 / 10 \text { I }-9.5\end{array}$ & & & & & \\
\hline 1503 & $14 \mathrm{da}$ & $\begin{array}{l}\text { start } 16 \mathrm{~mm} \text { at } 26 \mathrm{ft} . \\
f-6.3\end{array}$ & & & & & \\
\hline 1503 & $14 d a$ & $\operatorname{exp.~}_{f-i 1} 28$ Leica $1 / 8 \mathrm{sec}$. & 1505 & 21.20 & 23.40 & 2.20 & bl.gr. \\
\hline 1510 & $2 I \mathrm{da}$ & $\begin{array}{l}\exp , 29 \text { Leica } 1 / 8 \mathrm{sec} . \\
\text { f-il. }\end{array}$ & 1505 & 21.20 & 24.40 & 3.20 & yellow \\
\hline 1517 & $28 \mathrm{da}$ & $\operatorname{expp}_{f-I I} 30$ Leica $1 / 8 \mathrm{sec}$ & & & & & \\
\hline $\begin{array}{l}1520 \\
1520\end{array}$ & $\begin{array}{ll}31 & d a \\
3 I & d a\end{array}$ & $\begin{array}{c}\text { secured } 35 \text { at } 730 \text { exp. } \\
16 \text { at } 54 \text { ft. }\end{array}$ & & & & & \\
\hline BWCC & $0 \mathrm{da}$ & start W wind & & & & & \\
\hline 1534 & $13 \mathrm{da}$ & start 350 exp. $1 / 10$ & & & & & \\
\hline $\begin{array}{l}1534 \\
1535\end{array}$ & $\begin{array}{l}13 \mathrm{da} \\
14 \mathrm{da}\end{array}$ & $\begin{array}{l}\text { start } 16 \text { mm } 55 \text { ft. } f-6.3 \\
\text { exp. } 31 \text { Leica } 1 / 8 \text { f }-11\end{array}$ & $\begin{array}{l}1535 \\
1535\end{array}$ & $\begin{array}{l}24.40 \\
24.40\end{array}$ & $\begin{array}{l}26.60 \\
28.00\end{array}$ & $\begin{array}{l}2.20 \\
3.60\end{array}$ & $\begin{array}{l}\text { bl.gr. } \\
\text { yollow }\end{array}$ \\
\hline $\begin{array}{l}1542 \\
1549 \\
1550\end{array}$ & $\begin{array}{l}21 \mathrm{da} \\
28 \mathrm{da} \\
\text { clock }\end{array}$ & $\begin{array}{ccccc}\exp & 32 & \text { Lejea } & 1 / 8 & f-11 \\
" & 33 & " & " 1 \\
" & 34 & " & " 1\end{array}$ & & & & & \\
\hline $\begin{array}{l}1553 \\
1553\end{array}$ & $\begin{array}{l}32 \mathrm{da} \\
32 \mathrm{da}\end{array}$ & $\begin{array}{l}\text { secured } 35 \mathrm{~mm} \text { at } 250 \mathrm{exp} . \\
" 16 \mathrm{~mm} \text { at } 83 \mathrm{ft} .\end{array}$ & & & & & \\
\hline$\frac{\text { BSWCC }}{1553}$ & $0 \mathrm{da}$ & start SW wind & & & & & \\
\hline 1607 & $14 \mathrm{da}$ & $\begin{array}{l}\text { start } 35 \mathrm{~mm} \text { at } 150 \text { exp. } \\
1 / 20 \quad=-9.5\end{array}$ & & & & & \\
\hline 1607 & $14 \mathrm{da}$ & $\begin{array}{l}\text { start } 16 \mathrm{~mm} \text { at } 0 \mathrm{ft} . \\
\mathrm{s}-6.3\end{array}$ & & & & & \\
\hline
\end{tabular}




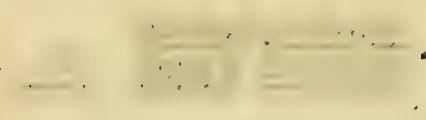

a..

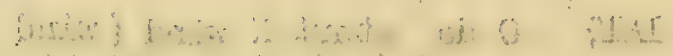

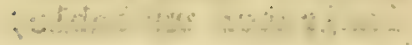

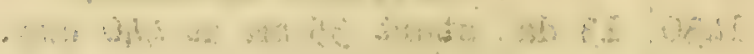

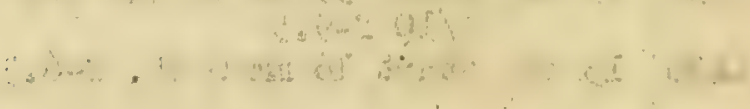

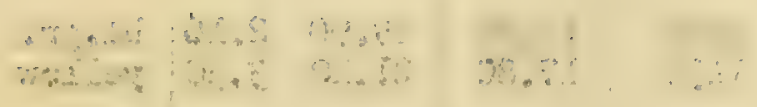

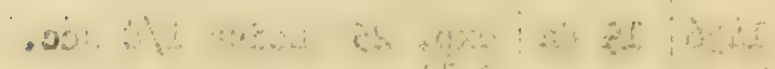

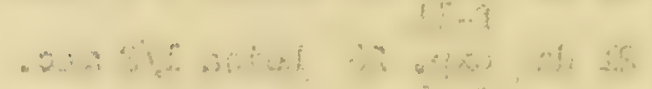

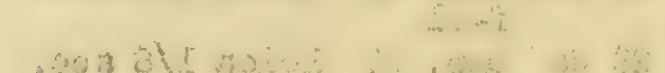

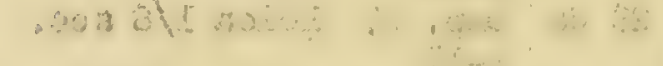

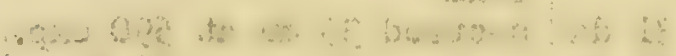

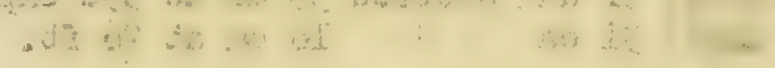

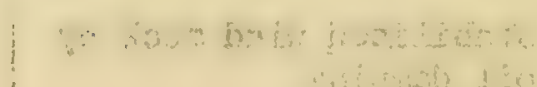
$\therefore$ in $\operatorname{lin}$

$\cdot \quad . \quad \ldots \quad$. $\therefore$

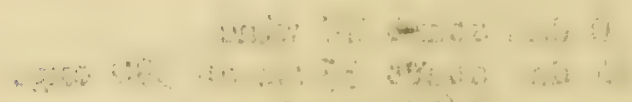

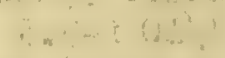
,$\because \therefore \therefore \therefore \therefore \therefore$

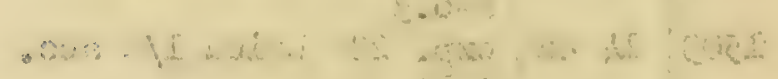

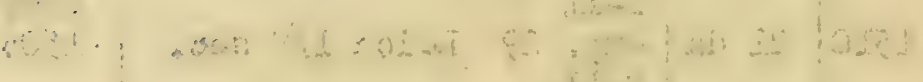

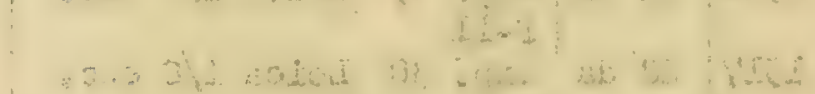

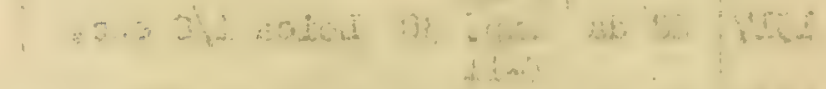

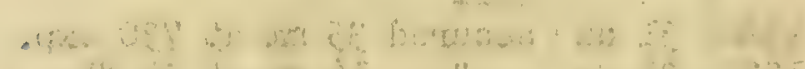

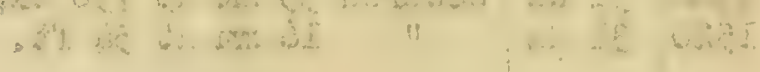

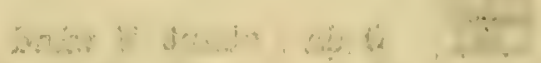

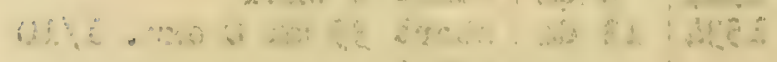
$\because \quad \quad \because \quad \quad \ldots, \quad, \ldots$

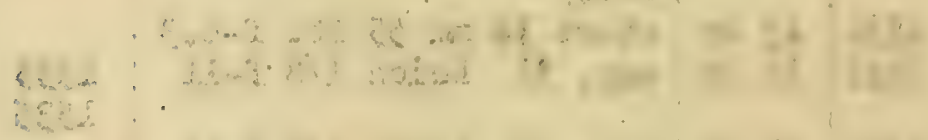

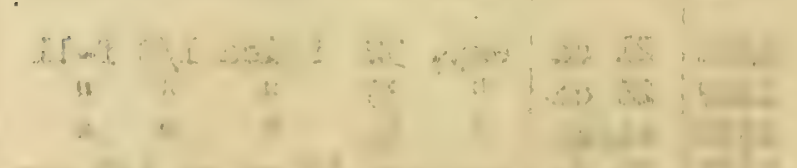

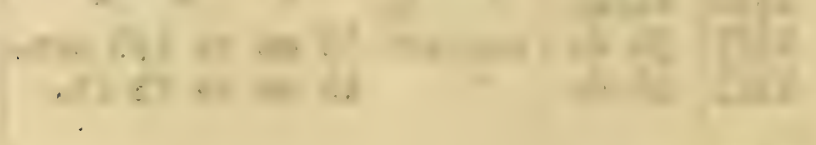




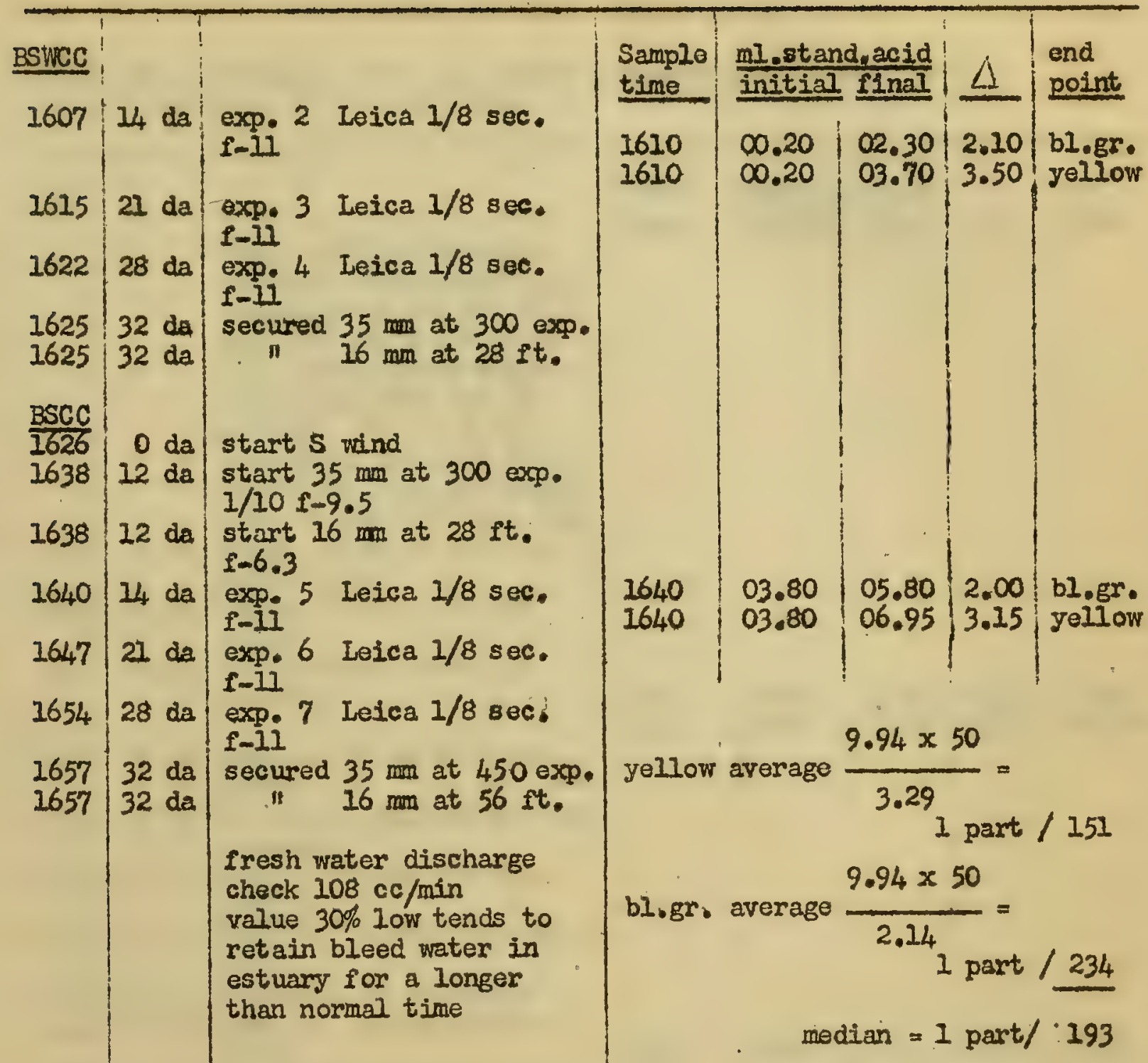




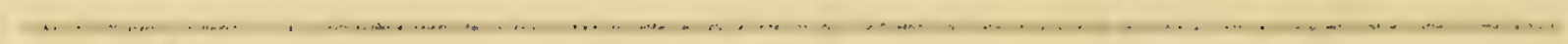

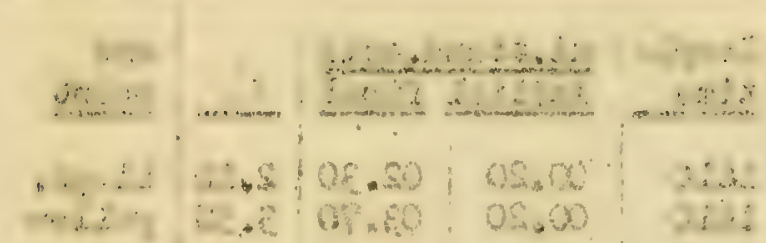

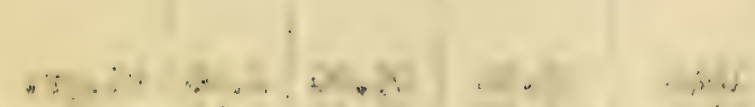
$\therefore \quad \therefore \quad \cdots \quad \cdots$

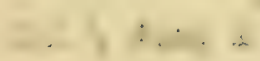

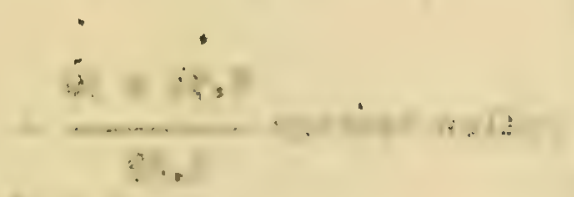

$\therefore \therefore \therefore$

$$
\therefore \therefore+1
$$$$
\text { i.. }
$$

$\because+\cdots$,

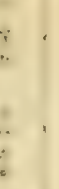

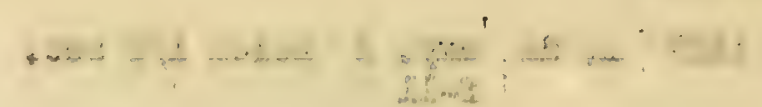

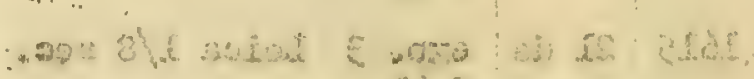

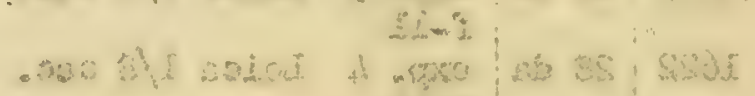
-

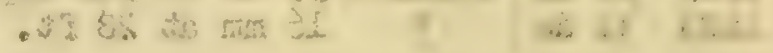

+
$\ddots$

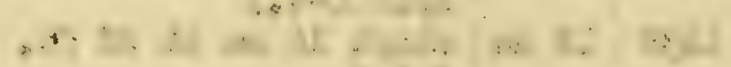
$\therefore \quad \therefore 1 \quad \therefore \quad \therefore \quad+1 \%$

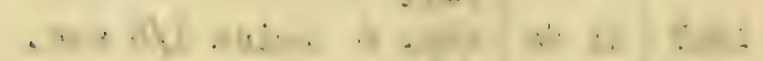

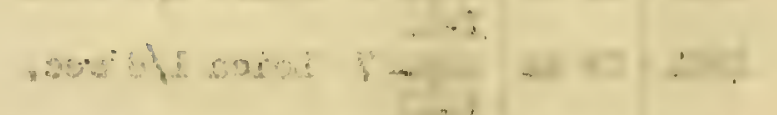

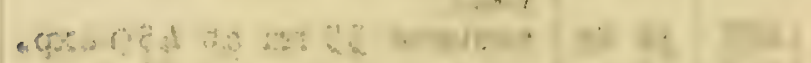

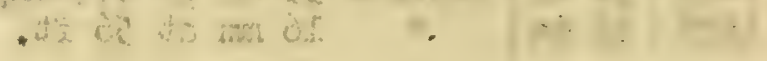

$\cdots \cdots$ $\cdots+20$ $\cdots \quad+\cdots$ $\therefore \quad \vdots \quad \cdots$ $\because \quad \because \cdots$ 
10 October 1949 TEST C _ CC

Bleed water discharge from all points (including dilution canal) characteristic of 1946 operations.

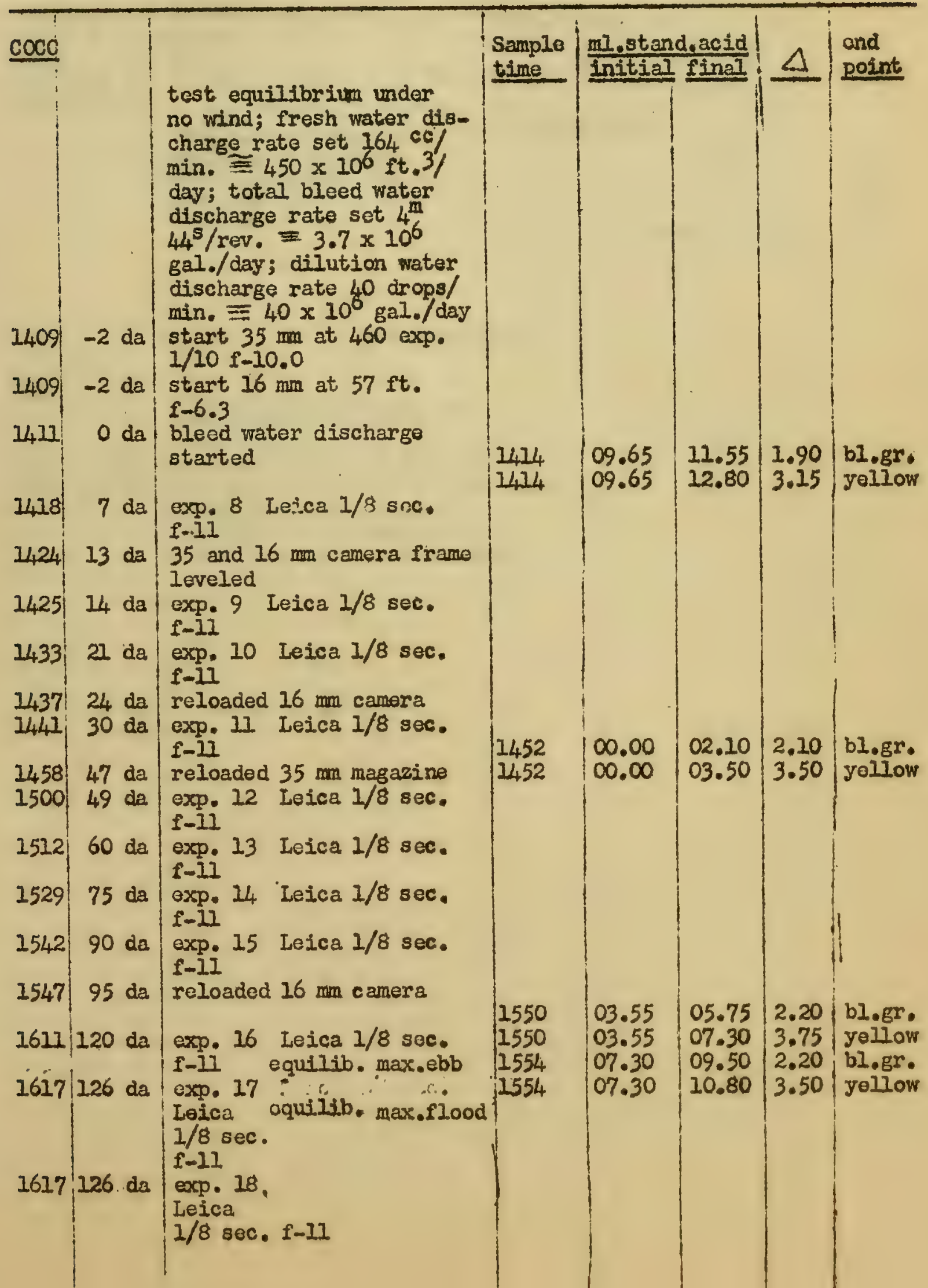




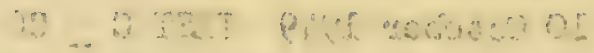

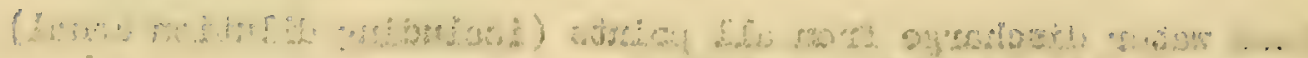

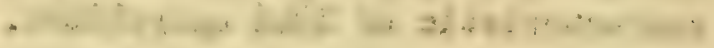

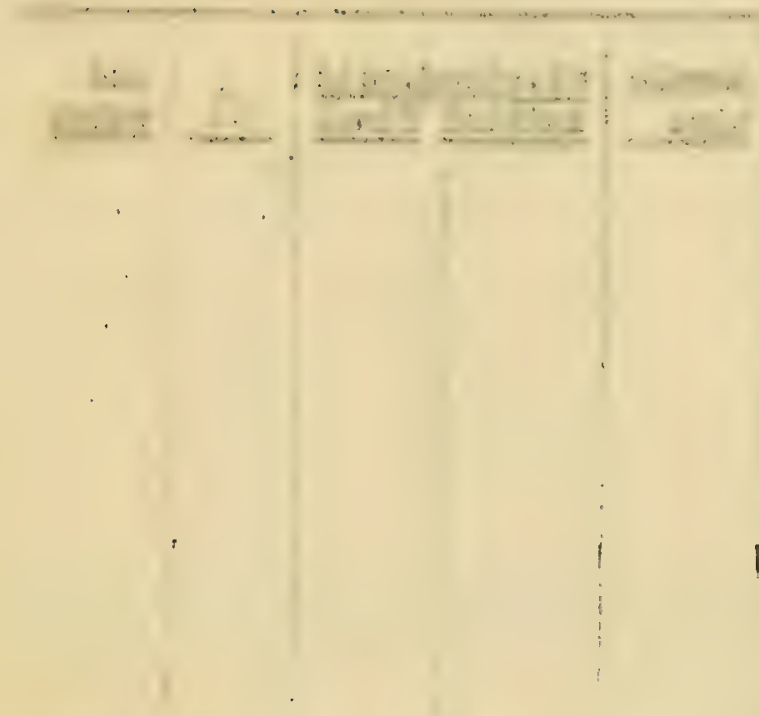

$\because, \ldots, \quad \because \ldots \quad \ldots+5$

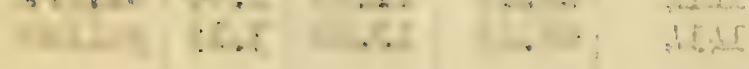
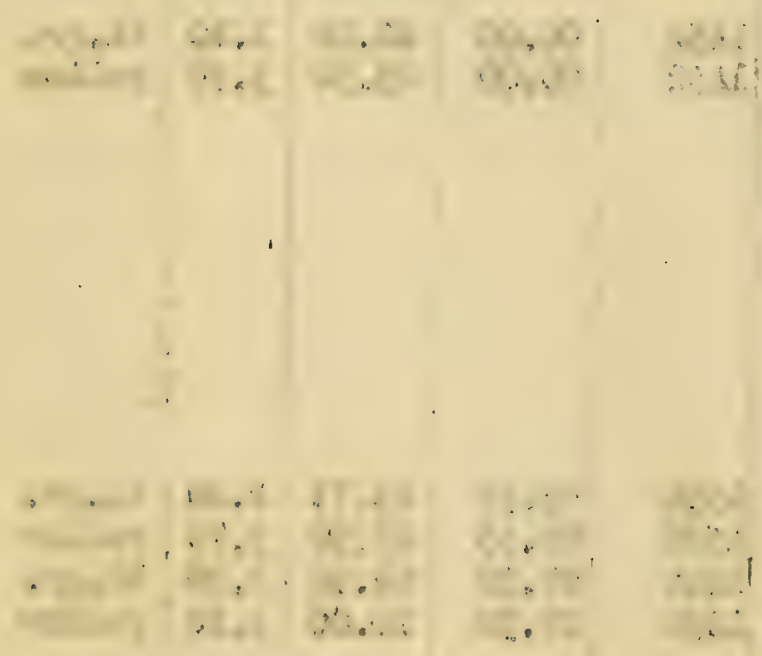

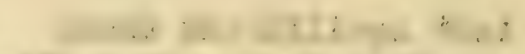

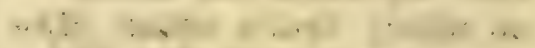

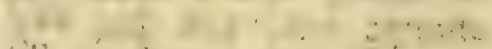

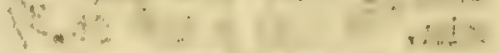 \\ un $\quad \therefore \quad \ldots \ldots$

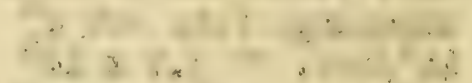

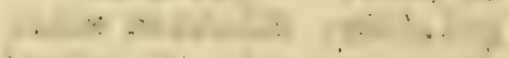

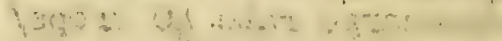 \\ $\because 30+15 \div 4=0$

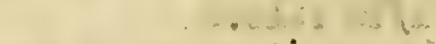

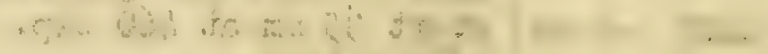

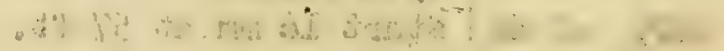
$\therefore<\rightarrow$

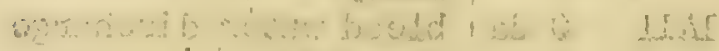
In j jonis is

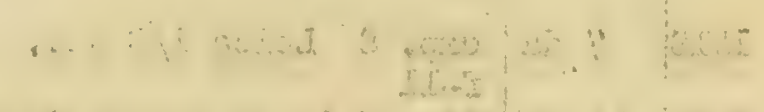

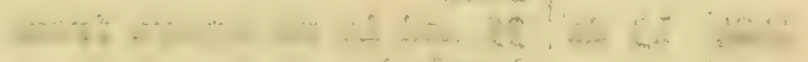

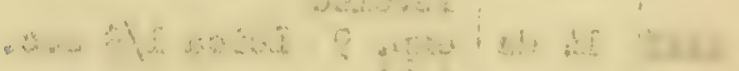

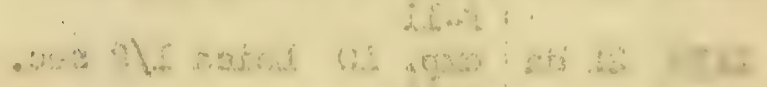

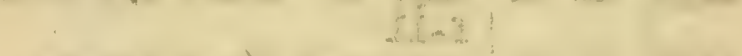

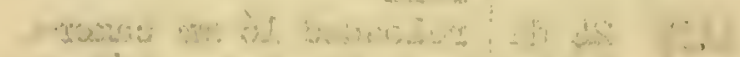

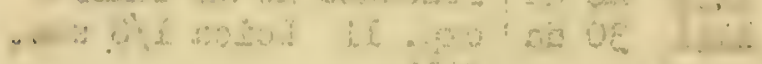

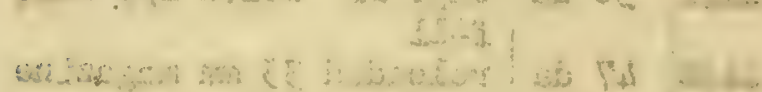
, कर , $\begin{array}{ll} & 0\end{array}$

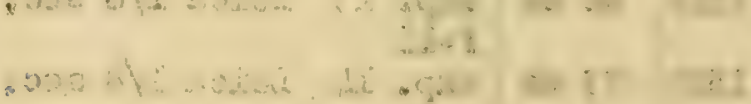

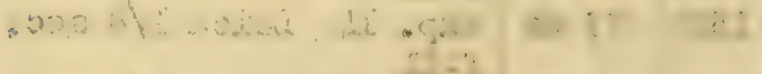

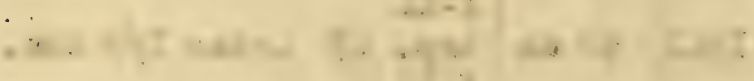
: $\quad+\quad+$

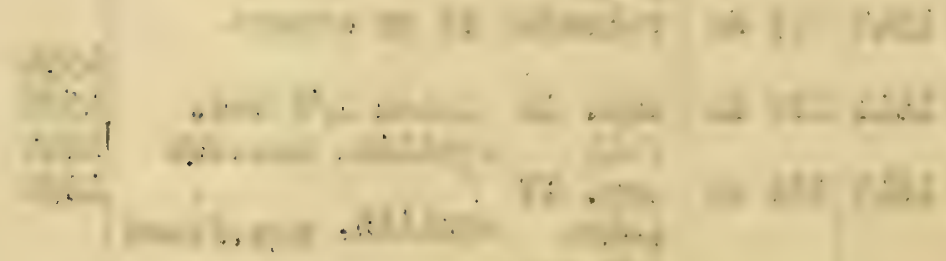




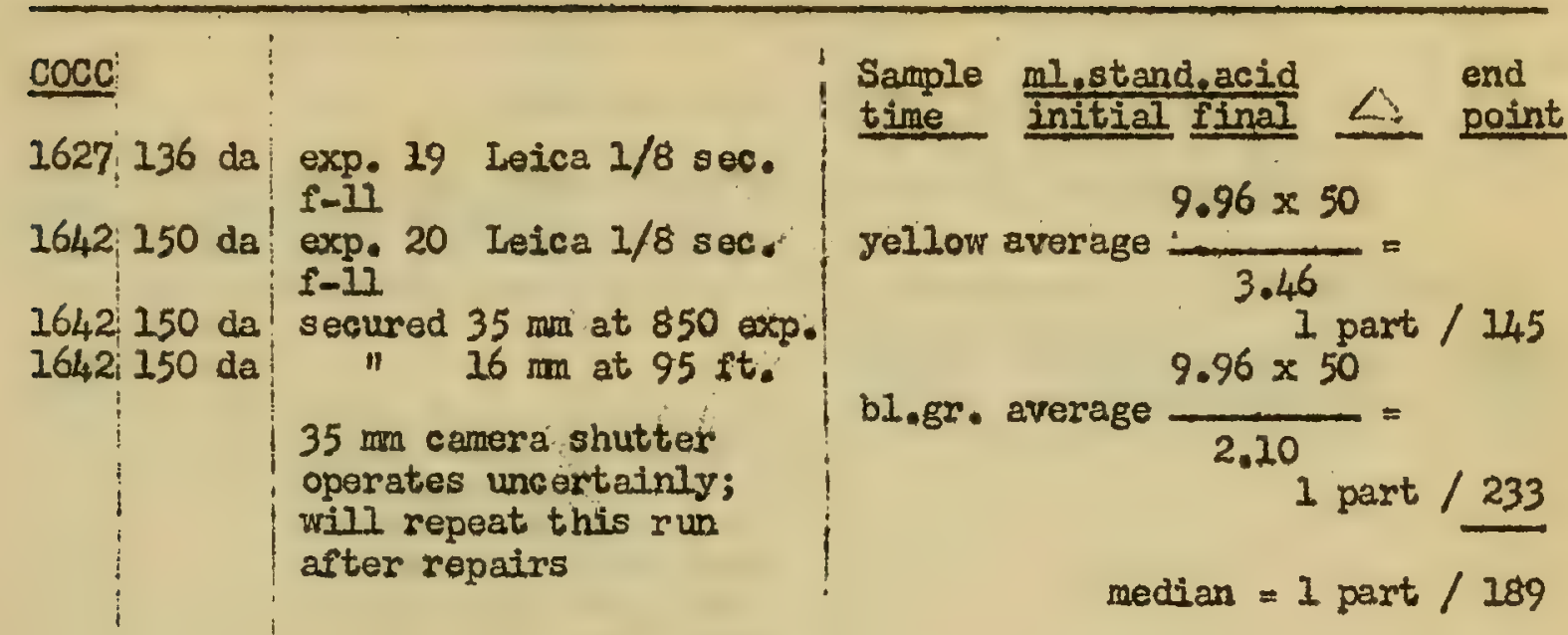

\section{October 1949}

Coce

1312 id 0 ran experiment 330 days but
1645 il took no photographs because repeat run conditions as on 10 October 1949 model behaved strangely (trouble later found to be draft from open loft windows) 


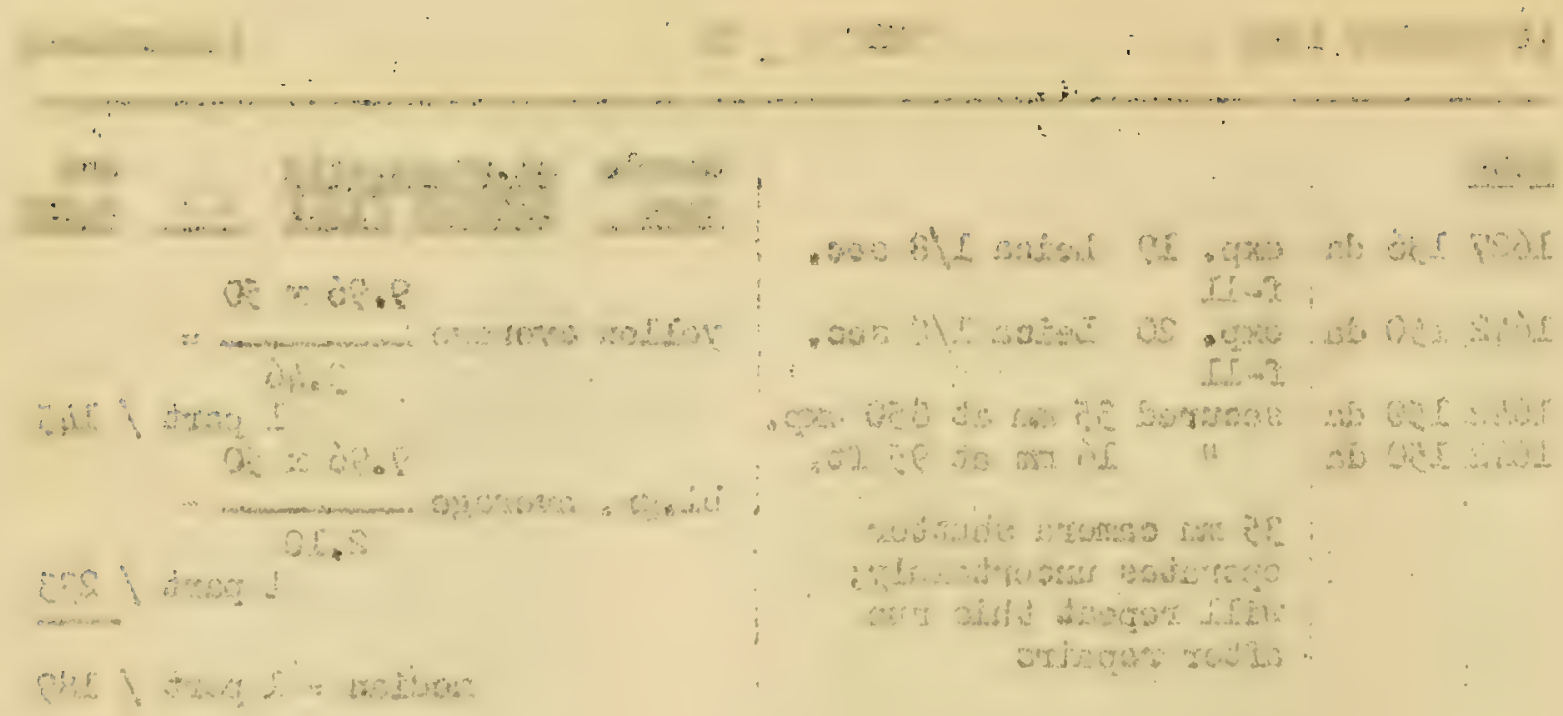

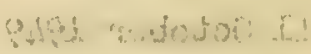

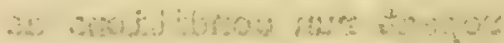

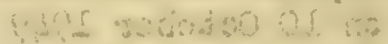

mi z

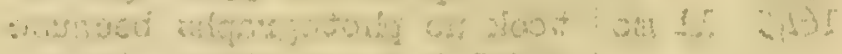

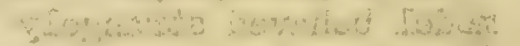

03

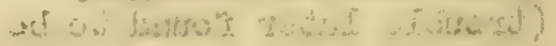

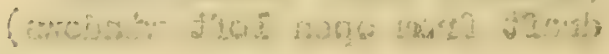


12 October 1949 TEST C _ CC

Bleed water discharge from all points (including dilution canal) characteristic of 1946 operations.

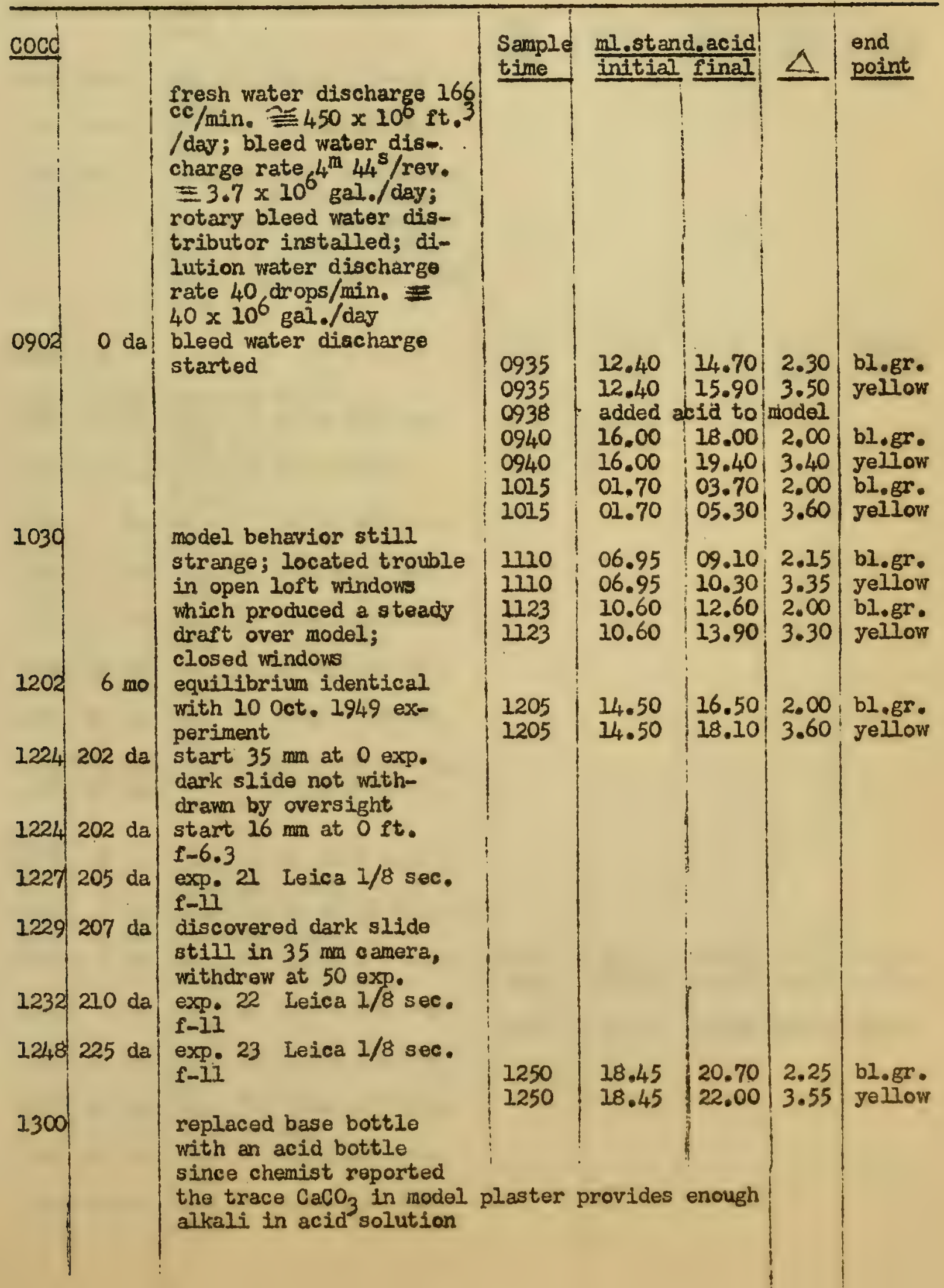




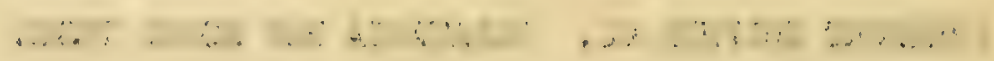

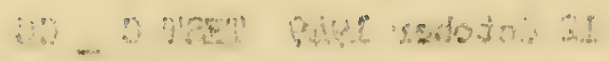

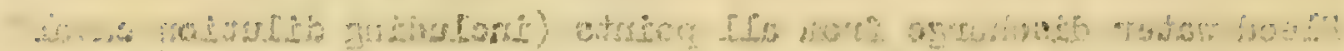

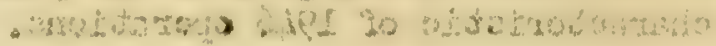

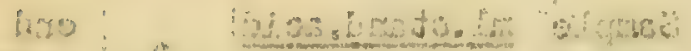

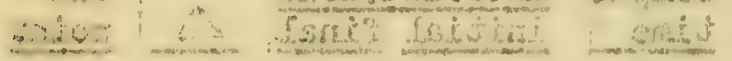

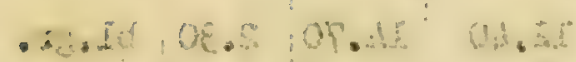

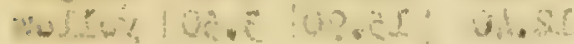

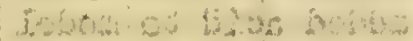
.7 .50 .

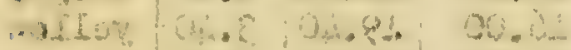

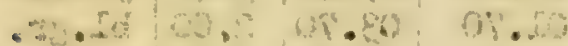

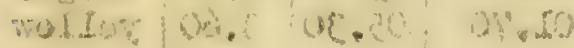

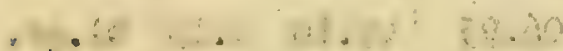
$\therefore \therefore \quad 6000$ . . D on 00.5

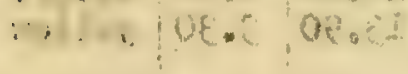

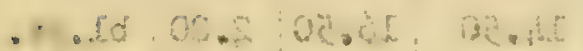

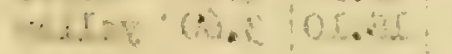

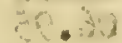

0.0 .6

03.0 ?

0.25
है?

360

30

औits

a)

TLE

$\because 105$

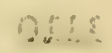

aldis

Esis ising

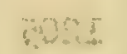

$\cos$

$\cdot \cdot$

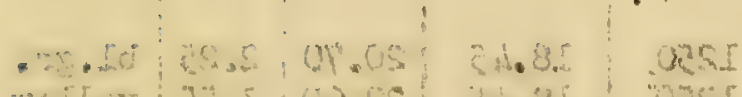

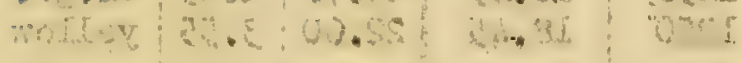

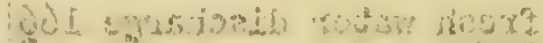

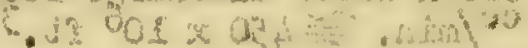

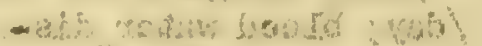

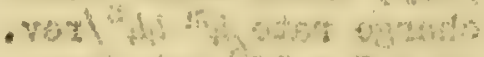

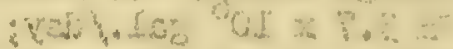

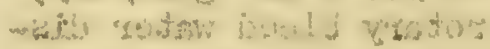

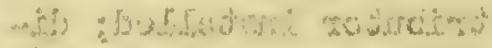

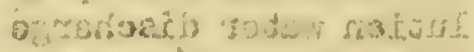

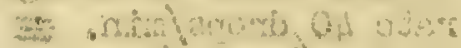
ए.

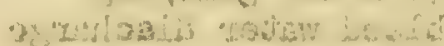

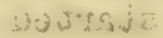

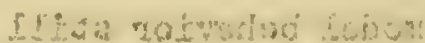

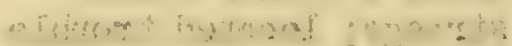

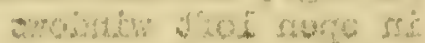

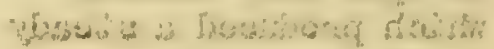

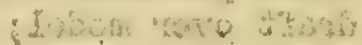

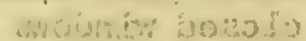

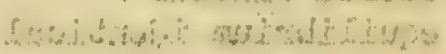
and a vestions

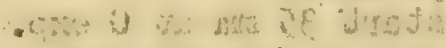

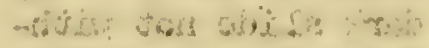

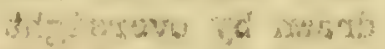

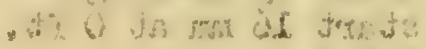

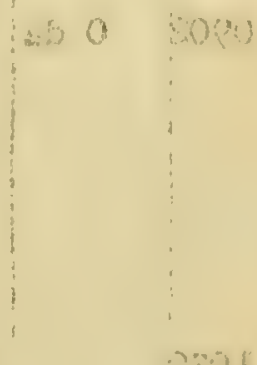

and
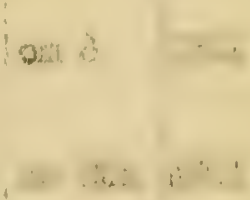

-

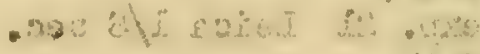
if

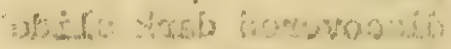

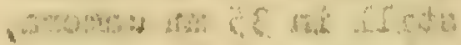
$\because \cdots, y$ a $\cdots, \cdots$ 5

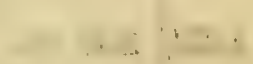

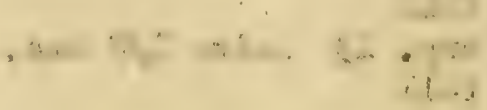
. . . . 
$\underline{\operatorname{cocd}}$

$1304240 \mathrm{da}$ exp. 24 Leica $1 / 8 \mathrm{sec}$. $1310246 \mathrm{da} \mid \begin{aligned} & \mathrm{f}-I I \\ & \exp .25\end{aligned}$ $1326263 \mathrm{da}$ exp. 26 Leica $1 / 8 \mathrm{sec}$. f-II

1330267 da exp. 27 Leica $1 / 8 \mathrm{sec}$. f-11

1330267 da secured 35 at 535 exp. $1330 \mid 267$ da test confirms 10 October 1949 and shutter worked well; synchronized I sec. late

CSRCC

1338 O da start $S R$ wind

1338 0 da start 35 at 535 exp. $1 / 10$ i -10

133810 da start $16 \mathrm{~mm}$ at $0 \mathrm{ft}$. $f-6.3$

1339 I da exp. 28 Leica $1 / 8$ sec. $1-1 i$

13447 da exp. 29 Leica $1 / 8 \mathrm{sec}$. i-ii

$135114 \mathrm{da}$ exp. 30 Leica $1 / 8 \mathrm{sec}$. f-11

$135821 \mathrm{da}$ exp. 31 Leica $1 / 8 \mathrm{sec}$. f-11

$140628 \mathrm{da}$ exp. 32 Leica $1 / 8$ sec. [-1]

$340931 \mathrm{da}$ secured $35 \mathrm{~mm}$ at 800 exp. 1409 $31 \mathrm{da}$ " $16 \mathrm{~mm}$ at $48 \mathrm{ft}$.

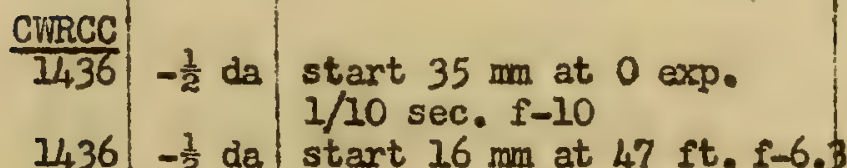
1436 - 0 da start $16 \mathrm{~mm}$ at $47 \mathrm{ft} .1-6.3$

$14403 \mathrm{da}$ exp. 33 Leica $1 / 8 \mathrm{sec}$.

$14447 \mathrm{da} \exp _{14} 34$ Leica $1 / 8 \mathrm{sec}$. f-ii

145214 da $\exp , 35$ Leica $1 / 8 \mathrm{sec}$. I-II

$145315 \mathrm{da} \exp .36$ Leica $1 / 8 \mathrm{sec}$. i-ii (clock)

$150224 \mathrm{da}$ exp. 1 Leica $1 / 8 \mathrm{sec}$. f-1]

$150628 \mathrm{da}$ exp. 2 Leica $1 / 8 \mathrm{sec}$. fin
Sample ml.stand.acid

time

initial final !

end

point

1323

1323

04.00

06.20 \begin{tabular}{l|l|l}
04.00 & 07.60
\end{tabular}

2.20

bl.gr.

3.60 yellow

07.80

10.00

2.20 bl.gx.

07.80

11.00

3.00

yellow
1440

1440

1446 $\infty .25$ 00.25

02.70

2.45

bl.gr. yellow 


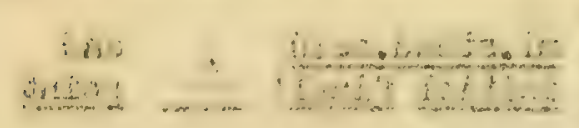
:

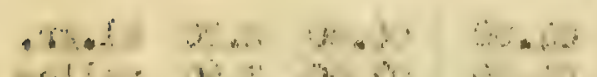

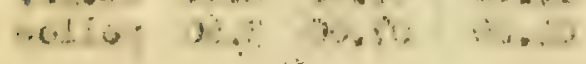

$\therefore$

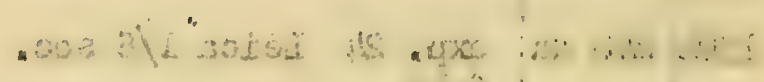

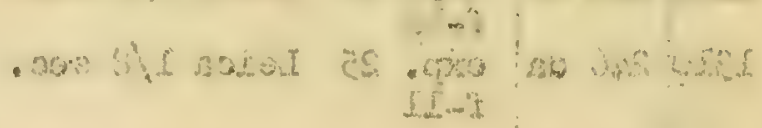
$\therefore$

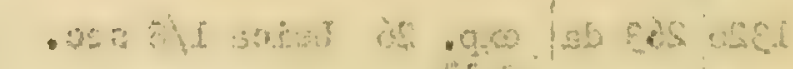

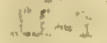

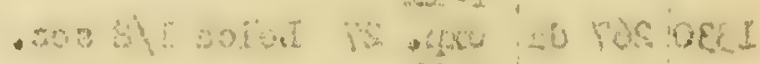
51.08

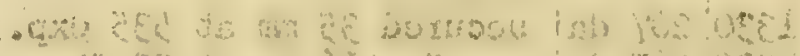

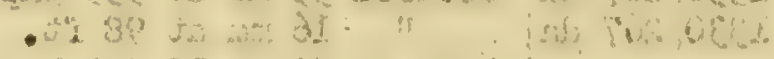

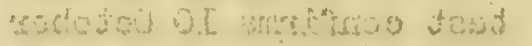

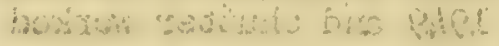

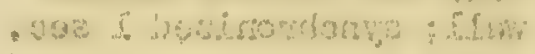
inis in

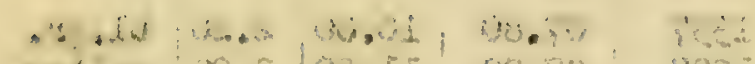

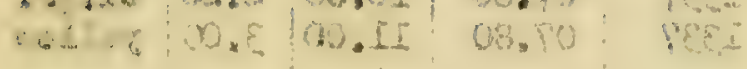

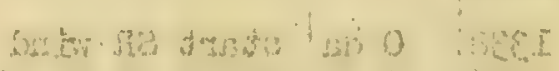

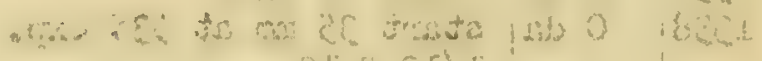
at ort $y$

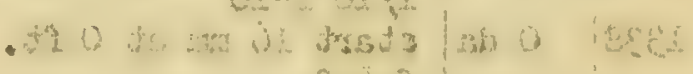

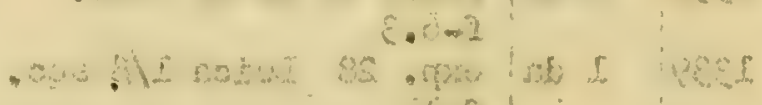

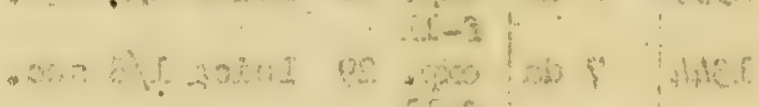

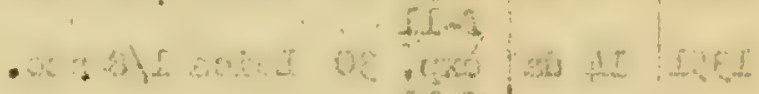

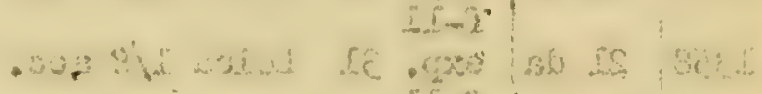

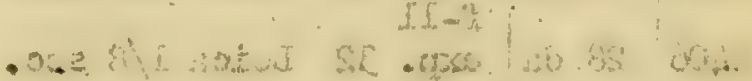

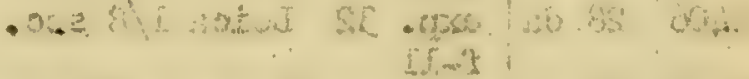

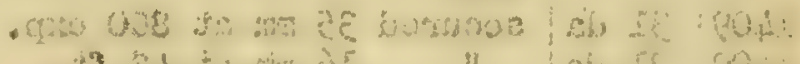

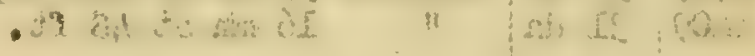
-

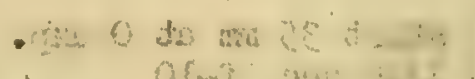

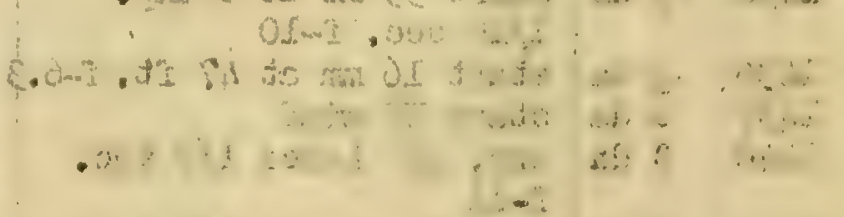

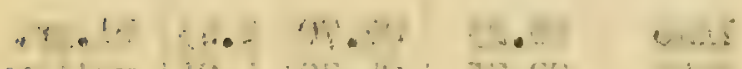

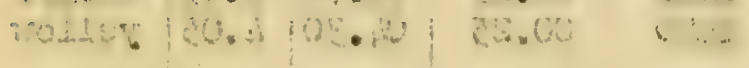

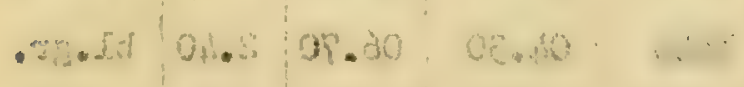
a.

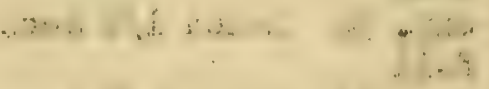

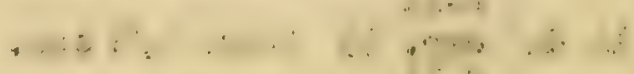

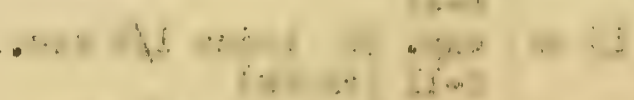

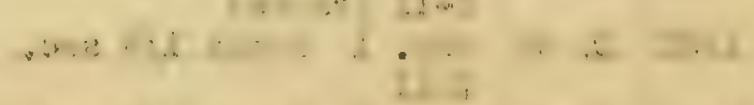

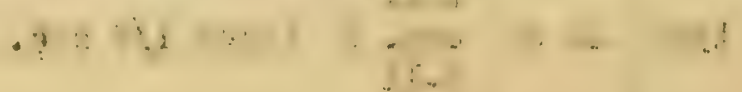




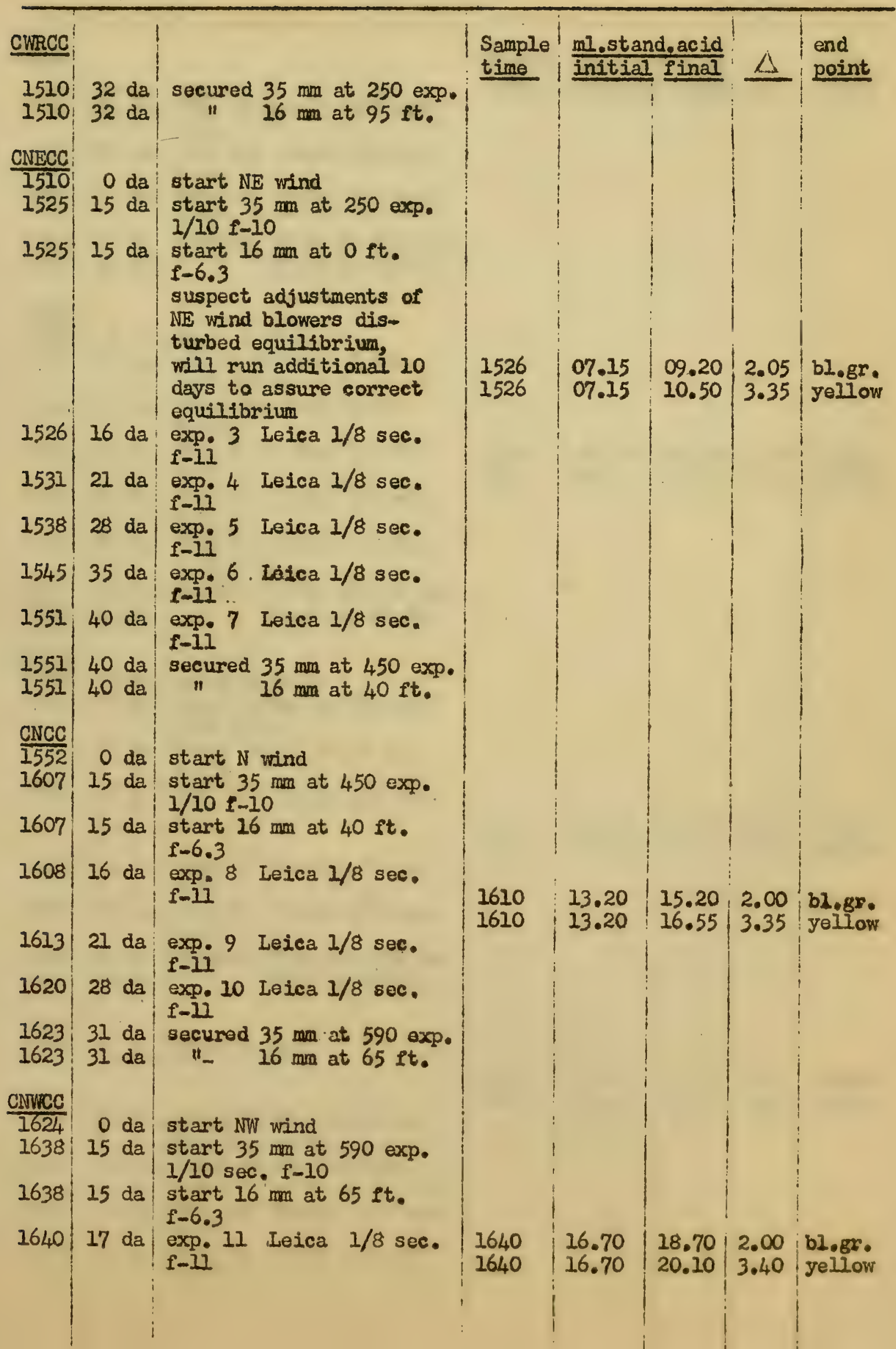


Who :

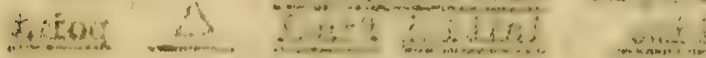

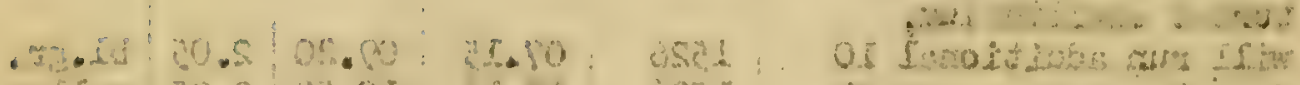

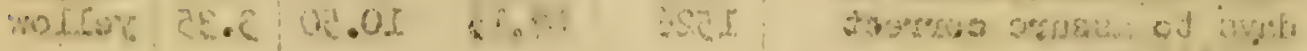

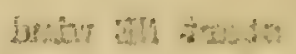
-

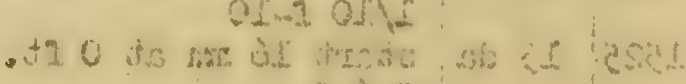
(5.2) -8

i.joring

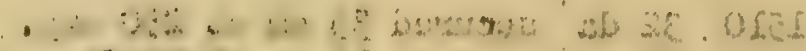

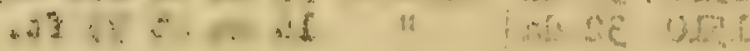

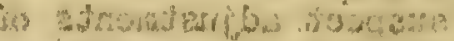

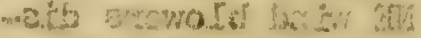

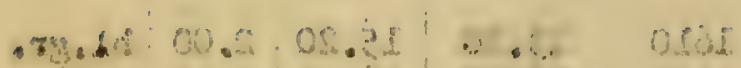

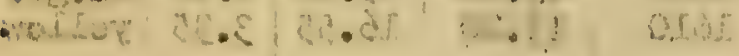
$\therefore$. 2 ri:

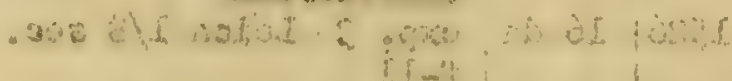

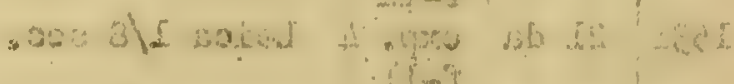

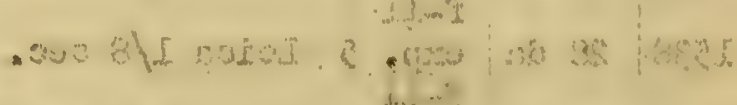

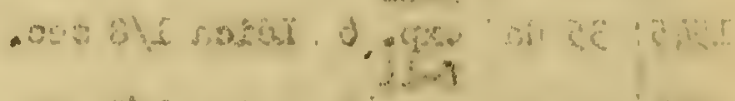

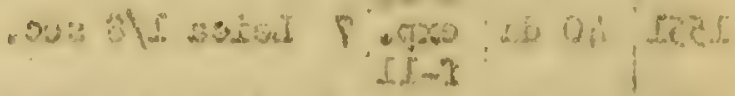

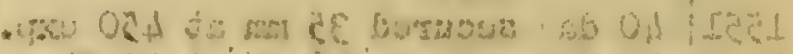

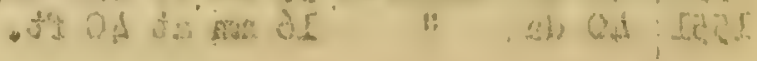

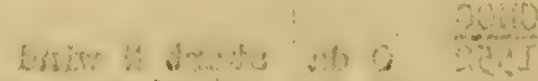

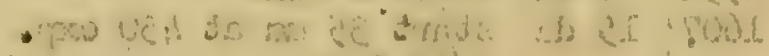

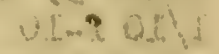

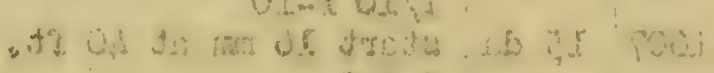

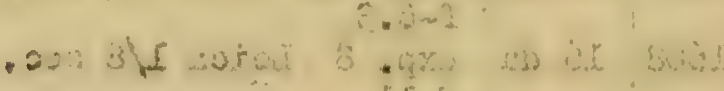

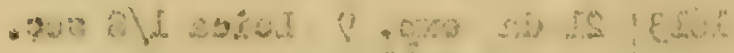
Sif

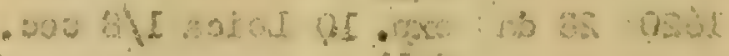
$i=-1$

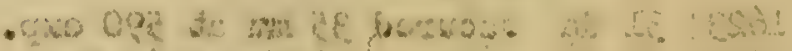

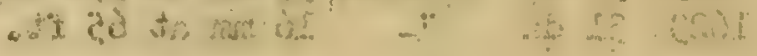

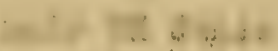

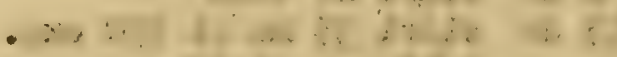

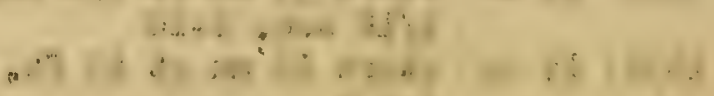
$\therefore \ldots$.

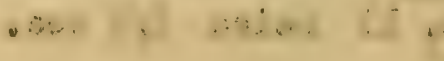




\begin{tabular}{|c|c|c|c|c|c|c|c|}
\hline CNwCe & & & $\begin{array}{l}\text { Sample } \\
\text { time }\end{array}$ & $\frac{\text { ml.stan }}{\text { Initial }}$ & $\frac{\text { d.acid }}{\text { final }}$ & $\Delta$ & $\begin{array}{l}\text { end } \\
\text { point }\end{array}$ \\
\hline 1644 & $2 I \mathrm{da}$ & $\begin{array}{l}\exp _{f-1 .} 12 \text { Leica } 1 / 8 \text { sec. } \\
\text { f }\end{array}$ & & & & & \\
\hline 1652 & $28 \mathrm{da}$ & $\exp _{f-1 i} 13$ Leica $1 / 8 \mathrm{sec}$ & & & & & \\
\hline 1659 & $35 \mathrm{da}$ & $\exp _{i-1 i} 14$ Leica $1 / 8 \mathrm{sec}$ & & & & & \\
\hline $\begin{array}{l}1700 \\
1700\end{array}$ & $\begin{array}{ll}36 & \mathrm{da}^{-1} \\
36 & \mathrm{da}\end{array}$ & $\begin{array}{c}\text { secured } 35 \mathrm{~mm} \text { at } 750 \text { exp. } \\
" 16 \mathrm{~mm} \text { at } 95 \mathrm{ft} .\end{array}$ & & & & & \\
\hline$\frac{\mathrm{CWCC}}{1700}$ & $O \mathrm{dal}$ & start W wind & & & & & \\
\hline 1713 & $13 \mathrm{da}$ & $\begin{array}{l}\text { start } 35 \mathrm{~mm} \text { at } 0 \text { exp. } \\
1 / 10 \mathrm{f}-10\end{array}$ & & & & & \\
\hline 1713 & $13 \mathrm{da}$ & $\begin{array}{l}\text { start } 16 \mathrm{~mm} \text { at } 0 \mathrm{ft} \text {. } \\
\mathrm{f}-6.3\end{array}$ & & & & & \\
\hline 1714 & $14 \mathrm{da}$ & $\exp _{f-1 I} 15$ Leica $1 / 8 \mathrm{sec}$. & $\frac{1724}{2734}$ & $\begin{array}{l}20.20 \\
20.20\end{array}$ & $\begin{array}{l}22.30 \\
23.70\end{array}$ & $\begin{array}{l}2.10 \\
3.50\end{array}$ & $\begin{array}{l}\text { bl.gr. } \\
\text { yellow }\end{array}$ \\
\hline 1721 & 21 da & exp, 16 Leica $1 / 8 \mathrm{sec}$ & & & & & \\
\hline 1728 & $28 \mathrm{da}$ & $\exp _{1-11} 17$ Leica $1 / 8 \mathrm{sec}$ & & & & & \\
\hline $\begin{array}{l}1731 \\
1731\end{array}$ & $\begin{array}{ll}31 & d a \\
31 & d a\end{array} \mid$ & $\begin{array}{c}\text { secured } 35 \mathrm{~mm} \text { at } 150 \mathrm{exp} . \\
16 \mathrm{~mm} \text { at } 29 \mathrm{ft} .\end{array}$ & & & 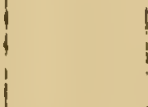 & & \\
\hline Cswec & & & & & & & \\
\hline $\begin{array}{l}1732 \\
1744\end{array}$ & $\begin{array}{r}0 \mathrm{da} \\
13 \mathrm{da}\end{array}$ & $\begin{array}{l}\text { start } S W \text { wind } \\
\text { start } 35 \mathrm{~mm} \text { at } 150 \text { exp. }\end{array}$ & & & & & \\
\hline 1744 & $13 \mathrm{da}$ & $\begin{array}{l}1 / 10 \mathrm{f}-10 \\
\text { start } 16 \mathrm{~mm} \text { at } 30 \mathrm{ft} . \\
\mathrm{f}-6.3\end{array}$ & & & & & \\
\hline 1745 & $u_{4}$ da & $\exp _{1-1]} 18$ Leica $1 / 8 \mathrm{sec}$. & 1747 & 01.80 & 03.90 & 2.10 & bl.gr. \\
\hline 1753 & $21 \mathrm{da}$ & $\exp _{f-1]} 19$ Leica $1 / 8 \mathrm{sec}$ & 1747 & 01.80 & 05.15 & 3.35 & yellow \\
\hline 1800 & $28 \mathrm{da}$ & $\exp _{f-11} 20$ Leica $1 / 8 \mathrm{soc}$ & & & & & \\
\hline $\begin{array}{l}1804 \\
1804\end{array}$ & $\left|\begin{array}{ll}32 & \mathrm{da} \\
32 & \mathrm{da}\end{array}\right|$ & $\begin{array}{c}\text { secured } 35 \mathrm{~mm} \text { at } 300 \mathrm{exp} . \\
" \quad 16 \mathrm{~mm} \text { at } 60 \mathrm{ft} .\end{array}$ & & & & & \\
\hline$\frac{\operatorname{cscc}}{1805}$ & $O$ da & start $s$ wind & & & & & \\
\hline & & & $\begin{array}{l}1816 \\
1816\end{array}$ & $\begin{array}{l}06.20 \\
06.20\end{array}$ & $\begin{array}{l}08.30 \\
09.90\end{array}$ & $\begin{array}{l}2.10 \\
3.70\end{array}$ & $\begin{array}{l}\text { bl.gr. } \\
\text { yellow }\end{array}$ \\
\hline 1818 & $13 \mathrm{da}$ & $\begin{array}{l}\text { start } 35 \mathrm{~mm} \text { at } 150 \text { exp. } \\
1 / 10 \mathrm{sec} . \mathrm{f}-10\end{array}$ & & & & & \\
\hline 1818 & $13 \mathrm{da}$ & $\begin{array}{l}\text { start } 16 \mathrm{~mm} \text { at } 60 \mathrm{ft} . \\
\mathrm{f}-6.3\end{array}$ & & & & & \\
\hline 1819 & $14 \mathrm{da}$ & $\exp _{f-11} 21$ Leica $1 / 8 \mathrm{sec}$. & & & & & \\
\hline 1825 & $20 \mathrm{da}$ & $\begin{array}{l}\exp _{f-11} 22 \text { Leica } 1 / 8 \mathrm{sec} \\
\end{array}$ & & & & & \\
\hline
\end{tabular}




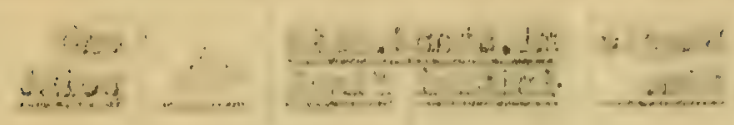

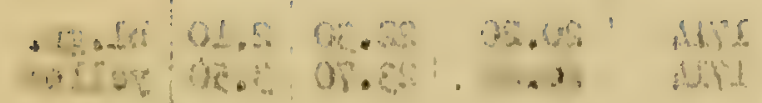

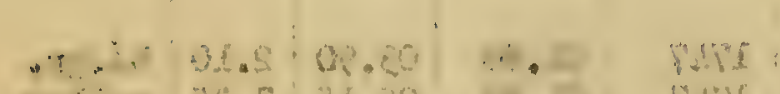
-

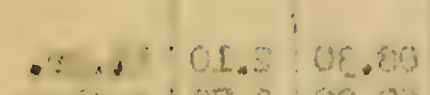
… T. T. Oe.

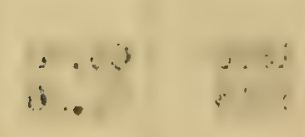

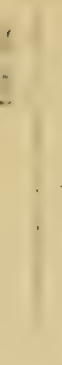

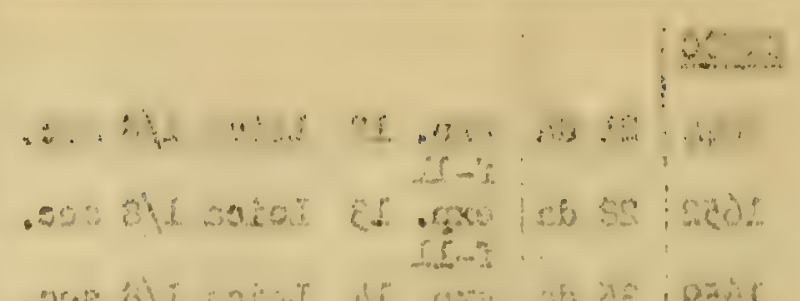

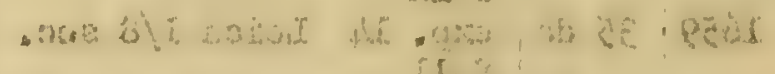

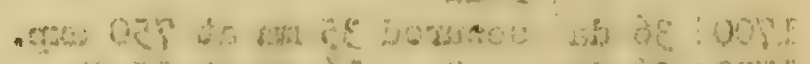

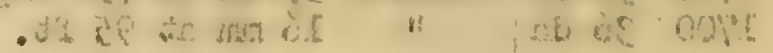

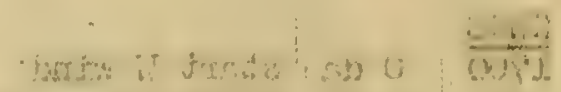

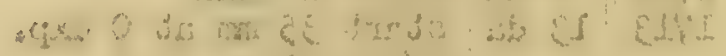

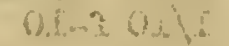

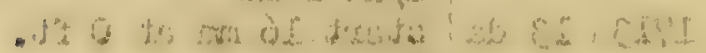 $8,-m$

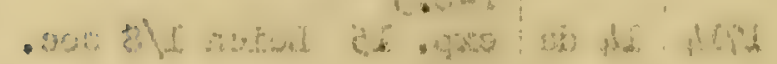

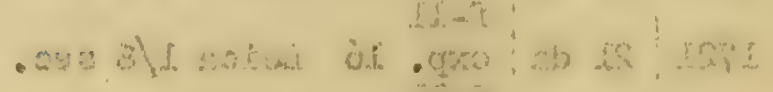

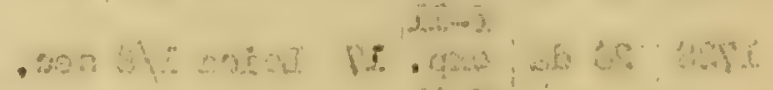

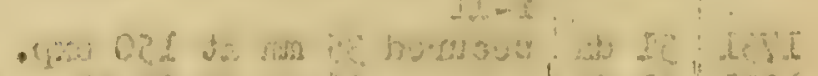

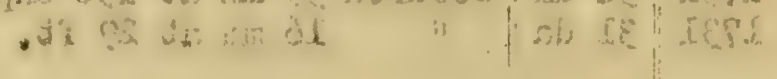

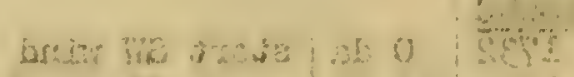

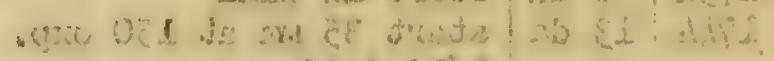

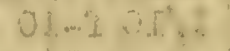

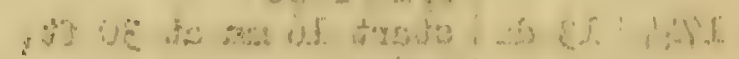

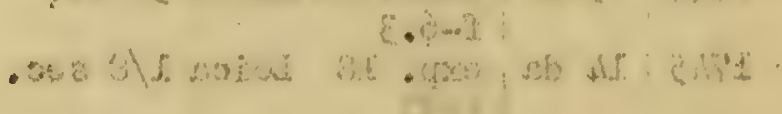

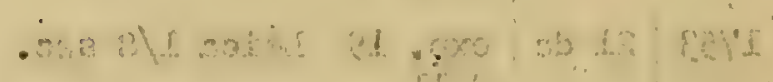

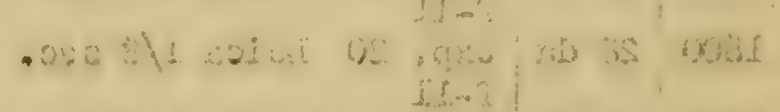

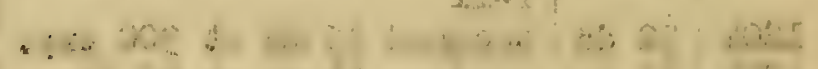
$x \div<\div \quad \because \quad, \quad, \quad, \ldots$

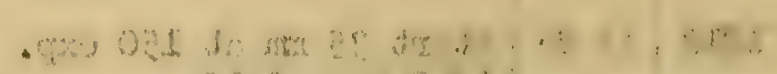
.0000000000 $\therefore \quad \because \quad \ldots \quad \therefore \cdots \cdots$

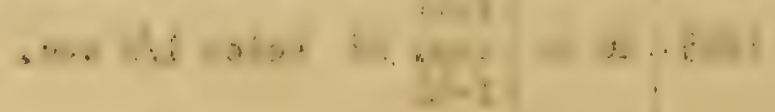




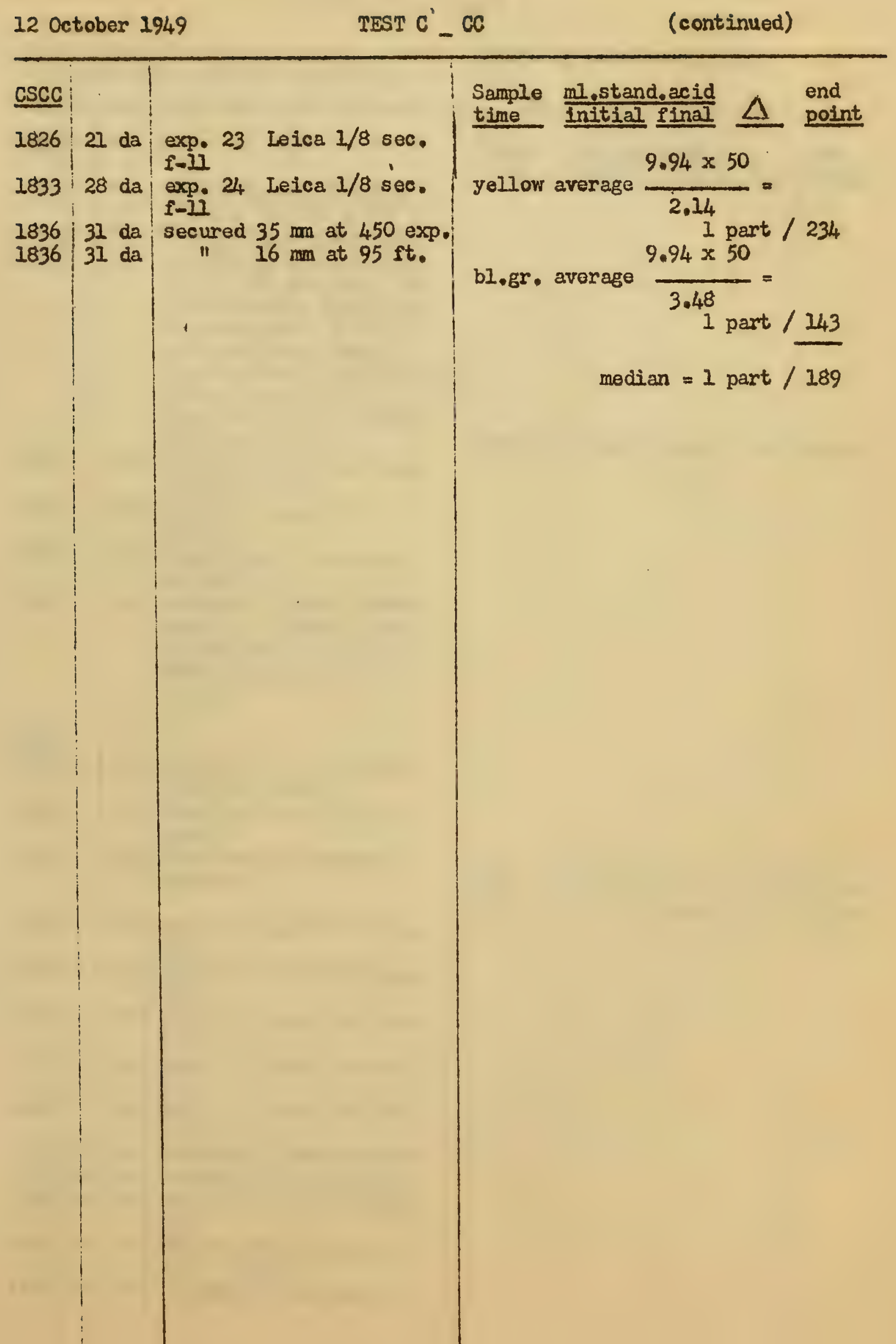




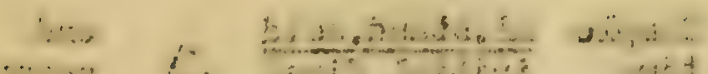
$\therefore$

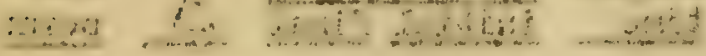

$$
\begin{aligned}
& \because \because \div, \cdots
\end{aligned}
$$

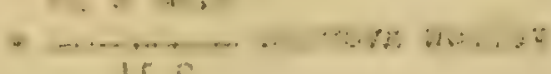

$$
\begin{aligned}
& 15.5
\end{aligned}
$$

thes to wing is

$$
\begin{aligned}
& 03=98
\end{aligned}
$$

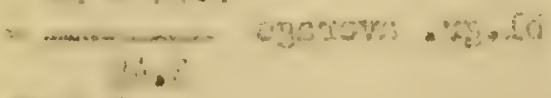

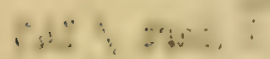

Q⿻日土龰

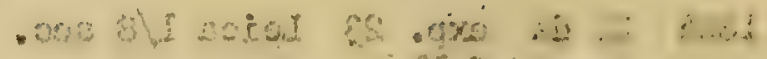

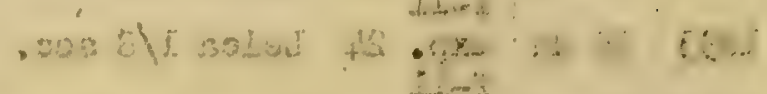

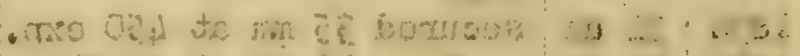

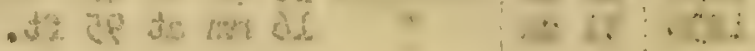




\section{October 1949 TEST C _ M}

Bleed water discharge from all points (including dilution sanal) characteristic of 1946 operations.

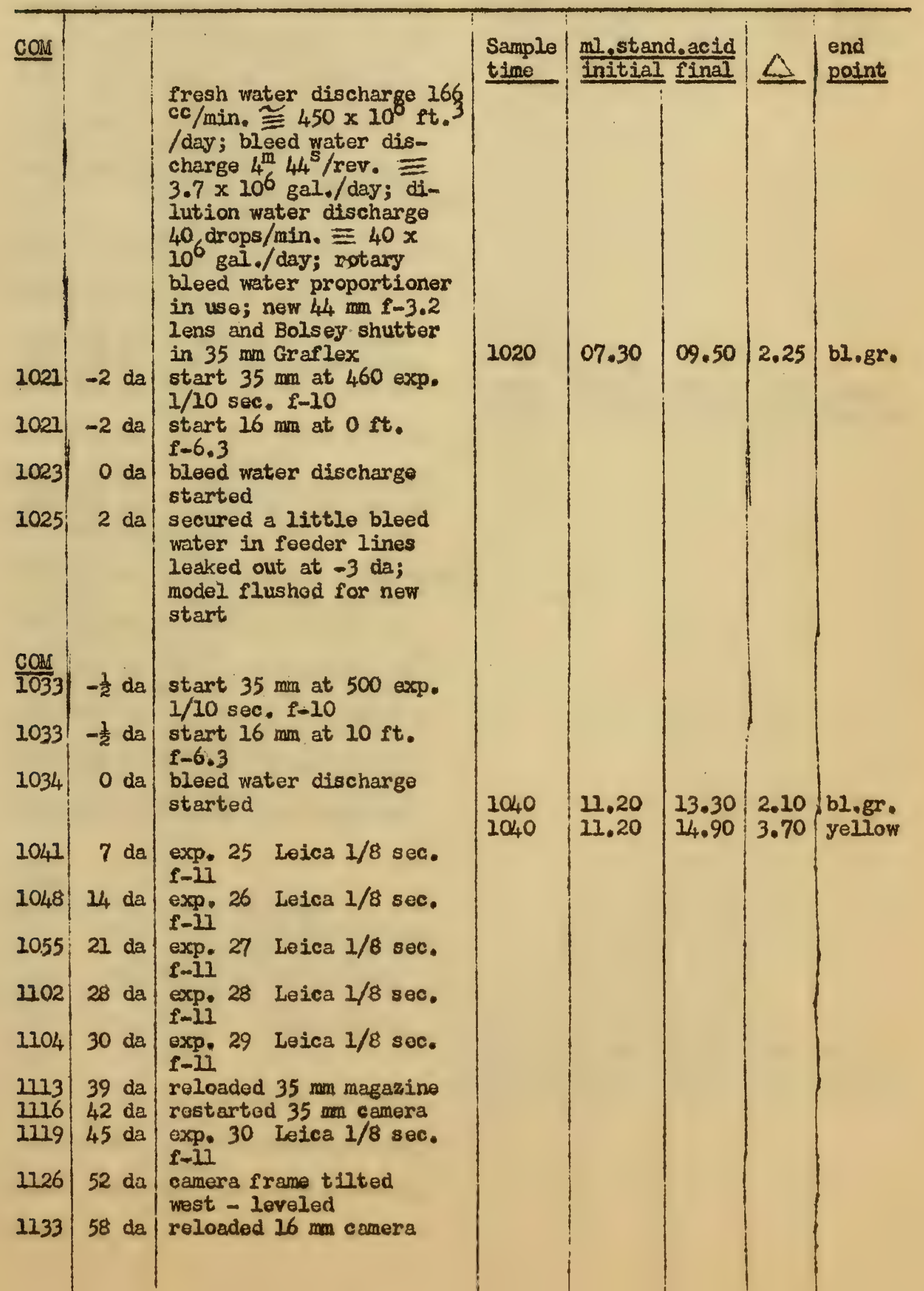




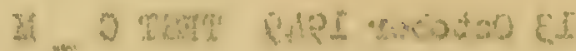

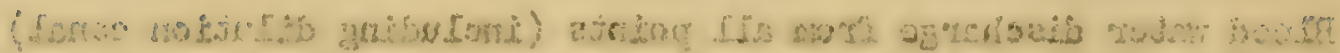

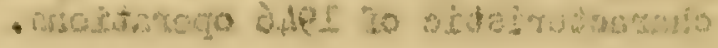

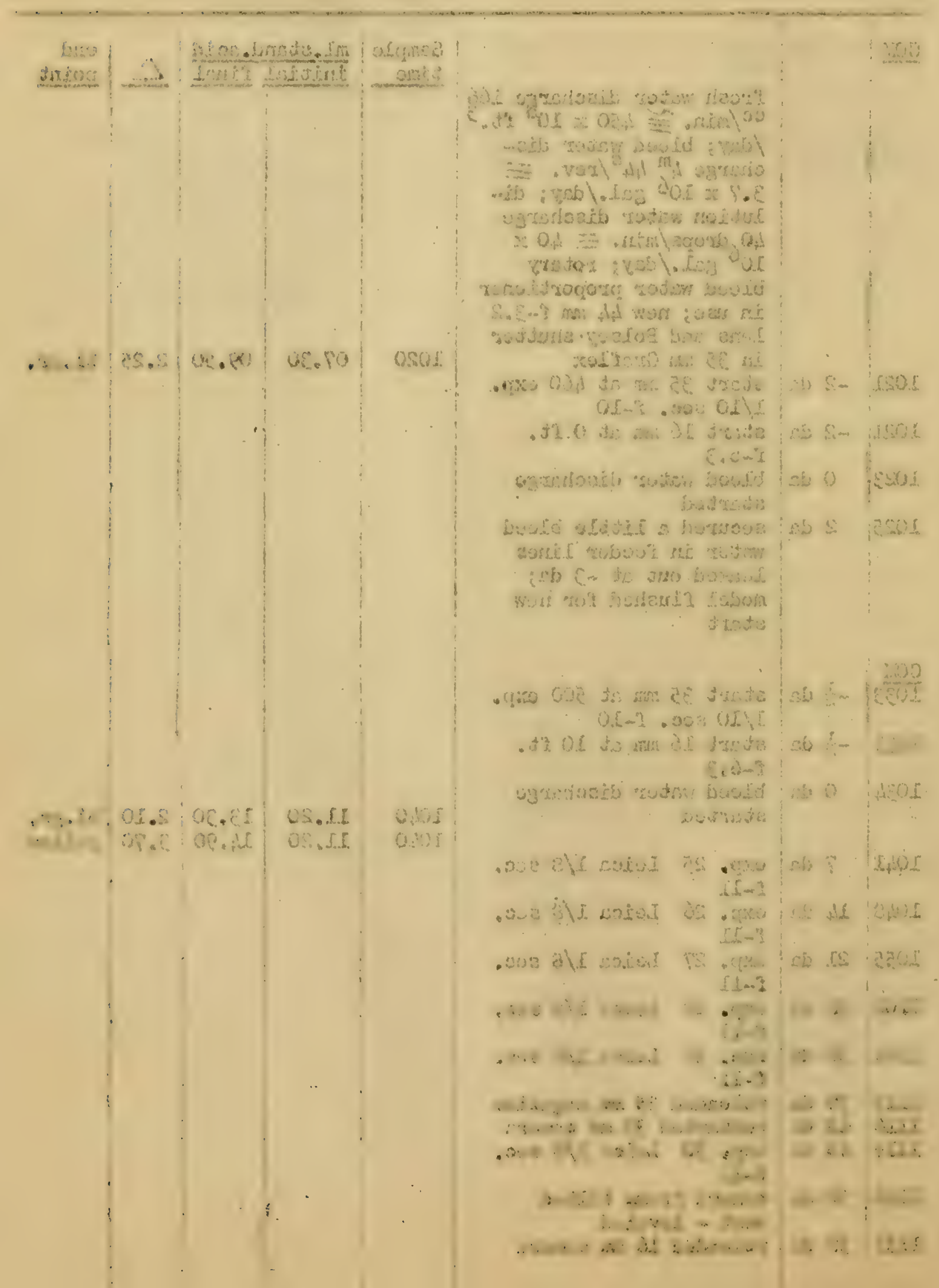




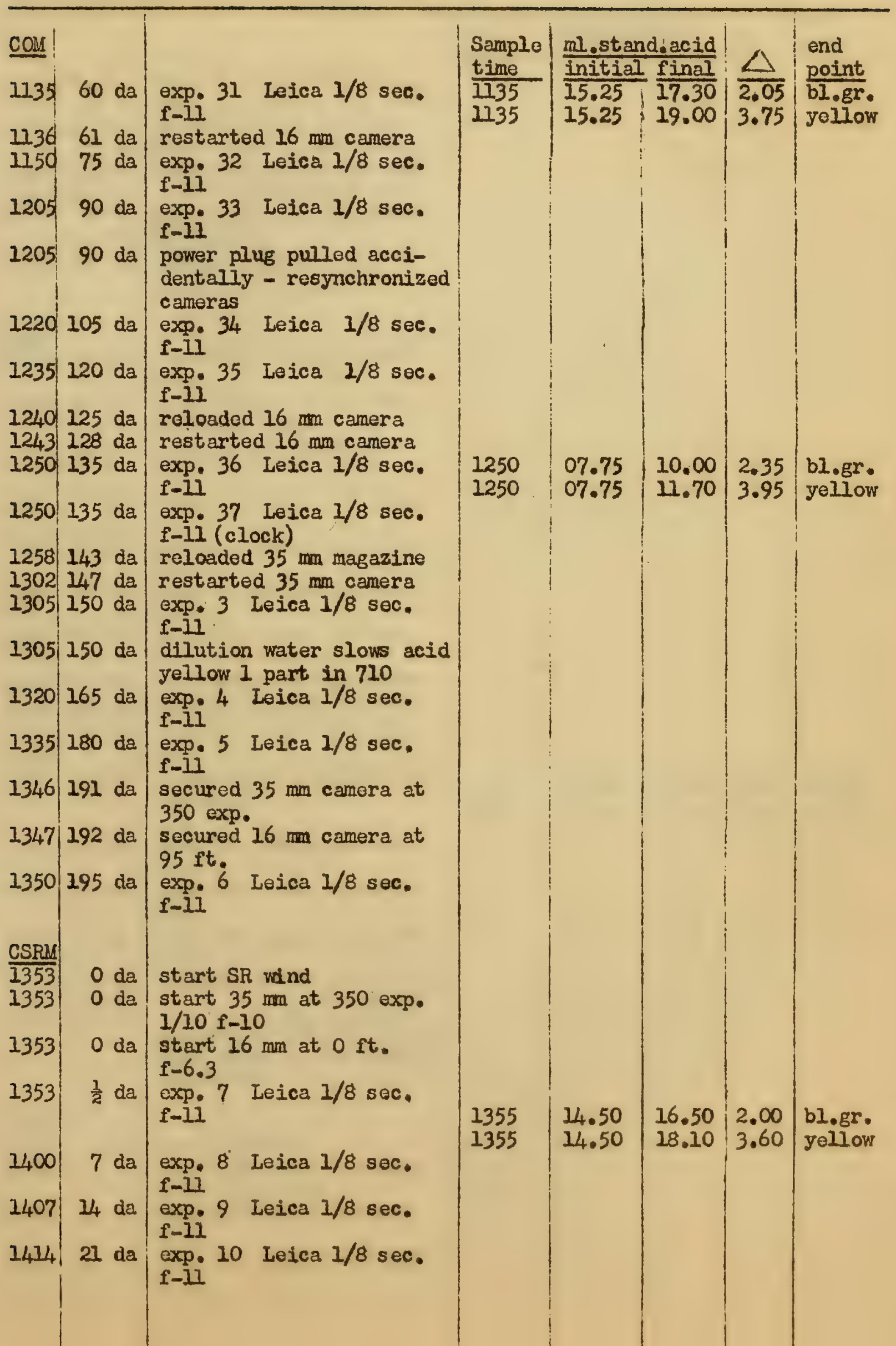


$\because \quad+\vdots \quad \therefore \quad \therefore-\div \quad \therefore$

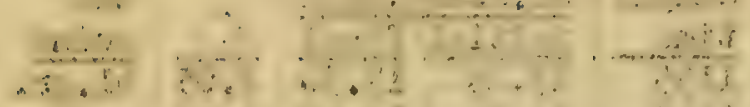

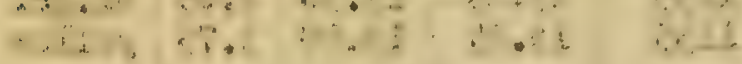

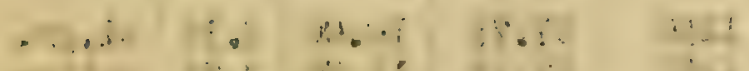
$\ldots, \therefore, \therefore, \ldots$

$\therefore \therefore \quad \therefore \quad \therefore \quad \therefore \quad \therefore \quad \therefore \quad \therefore$ $\therefore \therefore \therefore \quad \because \because \because \cdots \quad \therefore \quad \therefore \quad \therefore$

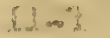

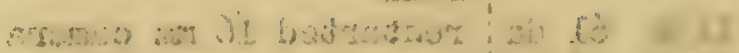

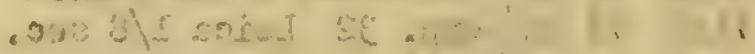
I. -1

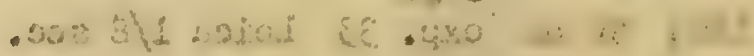
$5-2$

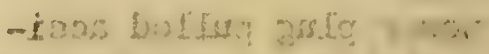

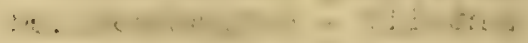

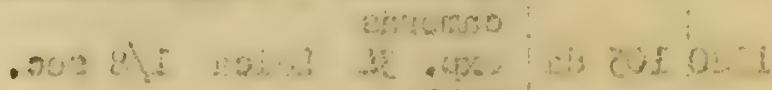

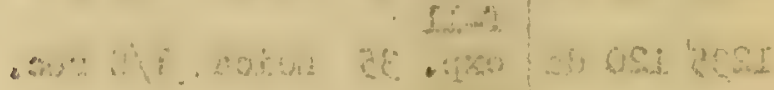

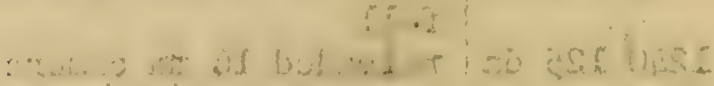

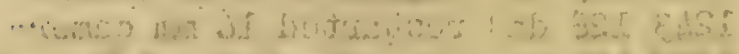
$\therefore \quad$ i $\quad \therefore$ a $\quad 01$

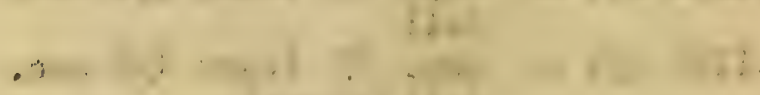

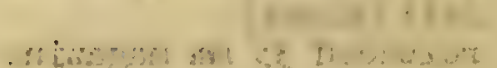

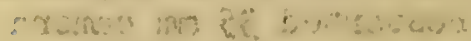

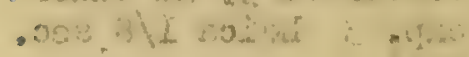
$\because$
$\vdots$
$\vdots$ -.....

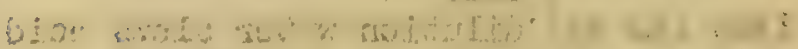

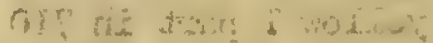

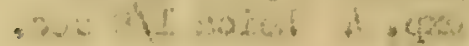
. . . . . . . . . . . . $, \ldots \ldots \ldots+i \ldots, \ldots, \ldots+\ldots$ - $\forall 1$

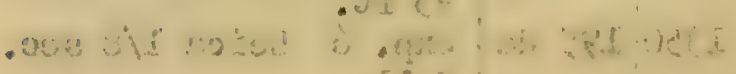
$\therefore \sum^{+\infty}$

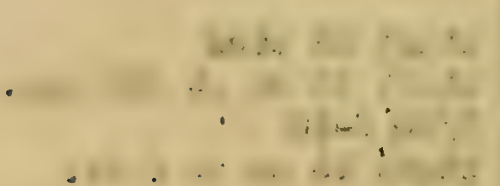
- $\quad . \quad \cdots \div$ $\therefore \because \quad \because \vdots \quad \cdots$ $\therefore \quad \div \quad \therefore \quad \cdots$, ㄴ, $\quad \therefore$. $\quad \because \therefore$,

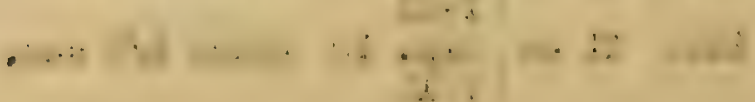




\begin{tabular}{|c|c|c|c|c|c|c|c|}
\hline CSRM & & & $\begin{array}{l}\text { Sample } \\
\text { time }\end{array}$ & $\frac{\text { mi.stan }}{\text { initial }}$ & $\frac{\text { d.acid }}{\text { final }}$ & $\Delta$ & $\begin{array}{l}\text { end } \\
\text { point }\end{array}$ \\
\hline 14,21 & 28 da & $\exp _{1-11}$ II Leica $1 / 8 \mathrm{sec}$ & & & & & \\
\hline$u_{423}$ & $30 \mathrm{da}$ & $\exp _{f-1 i}$ 12. Leica $1 / 8 \mathrm{sec}$. & & & & & \\
\hline $\begin{array}{l}1424 \\
1424\end{array}$ & $\begin{array}{l}31 \mathrm{da} \\
31 \mathrm{da}\end{array}$ & $\begin{array}{c}\text { secured } 35 \mathrm{~mm} \text { at } 600 \text { exp. } \\
n\end{array}$ & & & & & \\
\hline 1425 & & began return to no-wind & & & & & \\
\hline 1600 & & $\begin{array}{l}\text { no-wind equilibrium } \\
\text { mainly reestablished }\end{array}$ & & & & & \\
\hline RII & & & & & & & \\
\hline 502 & $0 \mathrm{da}$ & $\begin{array}{l}\text { start WR wind from } 1600 \\
\text { stage }\end{array}$ & & & & & \\
\hline 1614 & $12 \mathrm{da}$ & $\begin{array}{l}\text { start } 35 \text { mat at } 610 \text { exp. } \\
1 / 10 \text { sec. } f-10\end{array}$ & & & & & \\
\hline 1614 & & $\begin{array}{l}\text { start } 16 \mathrm{fm} \text { at } 48 \mathrm{ft} . \\
\mathrm{f}-6.3\end{array}$ & & & & & \\
\hline 1616 & & $\exp _{f-i 1} 13$ Leica $1 / 8$ sec. & & & & & \\
\hline 1623 & & $\begin{array}{l}\exp _{f-11} 14 \\
\text { Leica } 1 / 8 \mathrm{sec}\end{array}$ & & & & & \\
\hline 1630 & & $\begin{array}{l}\exp .15 \text { Leica } 1 / 8 \text { sec. } \\
f-1 i\end{array}$ & & & & & \\
\hline 1639 & $37 \mathrm{da}$ & $\exp _{f-1]} 16$ Leica $1 / 8 \mathrm{sec}$ & 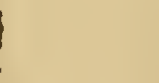 & & & & \\
\hline $\begin{array}{l}1641 \\
1644\end{array}$ & $\begin{array}{l}39 \mathrm{da} \\
43 \mathrm{da}\end{array}$ & $\begin{array}{l}\text { reloaded } 35 \mathrm{~mm} \text { magazine } \\
\text { restarted } 35 \mathrm{~mm} \text { camera }\end{array}$ & & & & & \\
\hline 1 & & $\exp _{\mathrm{f}-17} 17$ Leica $1 / 8 \mathrm{sec}$. & & & & & \\
\hline 16 & $48 \mathrm{da}$ & $\begin{array}{l}\text { reloaded and restartad } 16 \\
\text { mm camera. }\end{array}$ & $\begin{array}{l}1650 \\
1650\end{array}$ & $\begin{array}{l}01.70 \\
01.70\end{array}$ & $\begin{array}{l}03.70 \\
05.10\end{array}$ & $\begin{array}{l}2.00 \\
3.40\end{array}$ & $\begin{array}{l}\text { bl. } \\
\text { yeliow }\end{array}$ \\
\hline
\end{tabular}

$165149 \mathrm{da}$ exp. 18 Leica $I / 8 \mathrm{sec}$.

$1658 \quad 56$ da

exp. 19 Leica $1 / 8 \mathrm{sec}$. $f-1]$

$170260 \mathrm{da}$

exp. 20 Leica $1 / 8$ sec. f-II

170260 da

secured $35 \mathrm{~mm}$ at 150 exp.

170260 da

prolonged this run to get past cycle of asymmetrical tides

CNEM

$\overline{1704} 0$ da

start NE wind

1716

12 da

start $35 \mathrm{~mm}$ at 150 exp. $1 / 10$ sec. $f-10$

1726

12 da

start $16 \mathrm{~mm}$ at $20 \mathrm{ft}$.

$f-6.3$

$1 7 1 8 \longdiv { 1 4 } \mathrm { da }$

exp: 21 Leica $1 / 8 \mathrm{sec}$. \{-11 


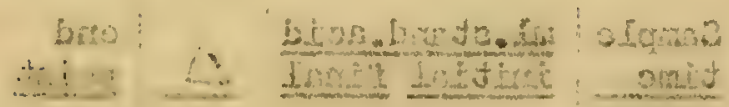

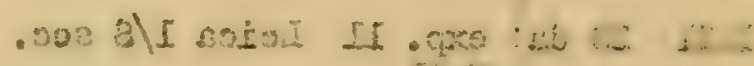
at.?

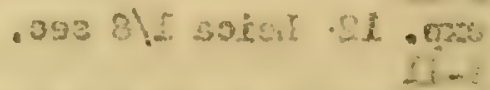
a. $\therefore$. त. - ace 60 मे ma a bexuger

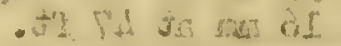

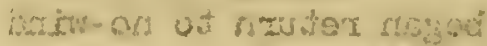
AL $\therefore U$

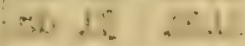

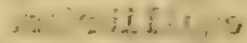
risinifidury ons on

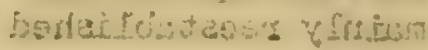

(6) is

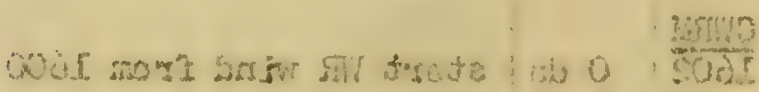

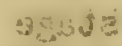

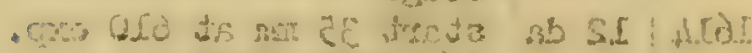

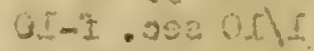

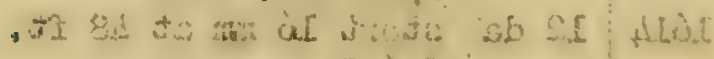

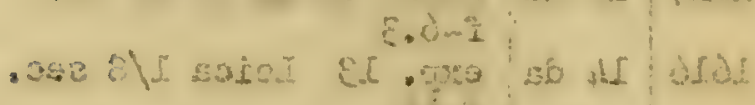

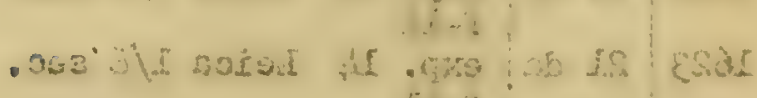

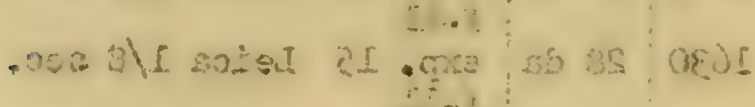

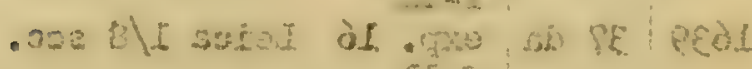

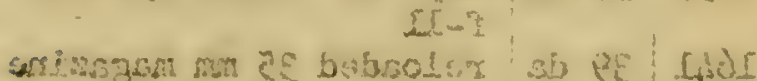

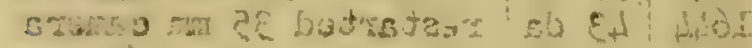

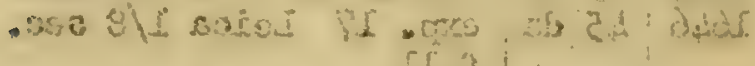

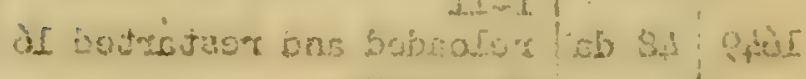

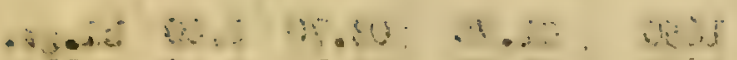

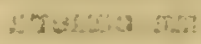

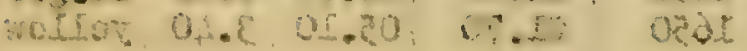

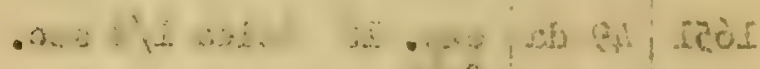
…?

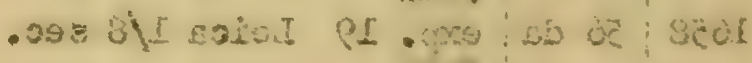

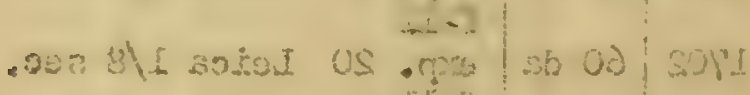
$1 \gamma_{0}+2$

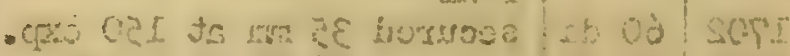

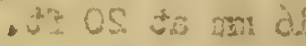
as cal sols

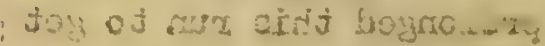

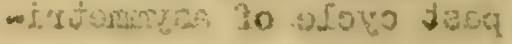
andistion

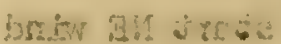

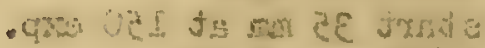

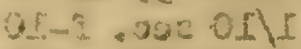

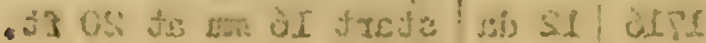

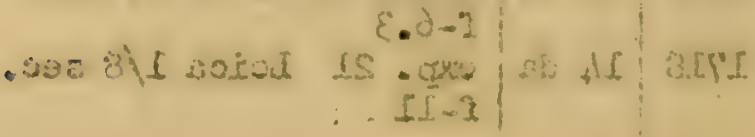




\begin{tabular}{|c|c|c|c|c|c|c|c|}
\hline CNEM & & & Sample & ml.stanc & dacid & & \\
\hline & & $\therefore$ & $\begin{array}{l}\frac{\text { time }}{1720} \\
1720\end{array}$ & $\begin{array}{l}\text { initial } \\
05.40 \\
05.40\end{array}$ & $\begin{array}{l}\frac{\text { final }}{07.35} \\
09.00\end{array}$ & $\frac{21}{1.95}$ & $\begin{array}{l}\text { point } \\
\text { bl.gr. } \\
\text { yellow }\end{array}$ \\
\hline 1725 & $21 \mathrm{da}$ & $\exp _{1-11} 22$ Leica $1 / 8 \mathrm{sec}$ & & & & & \\
\hline 1732 & $28 \mathrm{da}$ & $\exp _{f-11} 23$ Leica $1 / 8 \mathrm{sec}$ & & & & & \\
\hline $\begin{array}{l}1736 \\
1736\end{array}$ & $\begin{array}{ll}33 & d a \\
33 & d a\end{array}$ & $\begin{array}{c}\text { secured } 35 \mathrm{~mm} \text { at } 305 \mathrm{exp} . \\
\text { " } 16 \mathrm{~mm} \text { at } 50 \mathrm{ft} .\end{array}$ & & & & & \\
\hline$\frac{\text { CNMI }}{1745}$ & $0 \mathrm{da}$ & start $N$ wind, went to & & & & & \\
\hline 1845 & $60 \mathrm{da}$ & $\begin{array}{l}\text { supper } \\
\text { start } 35 \mathrm{~mm} \text { at } 305 \mathrm{exp} \text {. }\end{array}$ & & & & & \\
\hline 1845 & $60 \mathrm{da}$ & $1 / 10 \mathrm{sec} . \mathrm{f}-10$ & & & & & \\
\hline 1845 & $60 \mathrm{da}$ & $\begin{array}{l}I-0.3 \\
\exp _{1-1 i}\end{array} 24$ Leica $1 / 8$ sec. & 1850 & 09.50 & 11.66 & 2.10 & bl.gr. \\
\hline 1852 & $67 \mathrm{da}$ & $\exp _{[-1]} 25$ Leica $1 / 8 \mathrm{sec}$ & 1850 & 09.50 & 12.70 & 3.20 & yellow \\
\hline 1859 & 74 da & $\exp _{1-1 i} 26$ Leica $1 / 8 \mathrm{sec}$ & & & & & \\
\hline 1906 & 81 da & 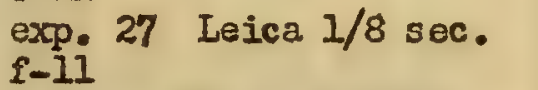 & & & & & \\
\hline $\begin{array}{l}1906 \\
1906\end{array}$ & $\begin{array}{l}81 \mathrm{da} \\
81 \mathrm{da}\end{array}$ & $\begin{array}{l}\text { secured } 35 \mathrm{~mm} \text { at } 480 \text { exp. } \\
\text { " } 16 \mathrm{~mm} \text { at } 83 \mathrm{ft} \text {. }\end{array}$ & & & & & \\
\hline$\frac{\text { CNWMI }}{1907}$ & 0 da & start NW wind & & & & & \\
\hline 1921 & 14 da & $\begin{array}{l}\text { start } 35 \mathrm{~mm} \text { at } 480 \text { exp. } \\
1 / 10 \mathrm{sec} . \mathrm{f}-10\end{array}$ & & & & & \\
\hline 1921 & $14 \mathrm{da}$ & $\begin{array}{l}\text { start } 16 \mathrm{~mm} \text { at } 0 \mathrm{ft} \text {. } \\
\mathrm{f}-6.3\end{array}$ & & & & & \\
\hline 1921 & $14 \mathrm{da}$ & $\exp _{f-I i} 28$ Leica $1 / 8 \mathrm{sec}$ & $\begin{array}{l}1925 \\
1925\end{array}$ & 13.00 & 15.00 & 2.00 & bl.gr. \\
\hline 2928 & $21 \mathrm{da}$ & $\exp _{1-1 i} 29$ Leica $1 / 8 \mathrm{sec}$ & & & & & \\
\hline 1935 & & $\exp _{1-I i} 30$ Leica $1 / 8 \mathrm{sec}$ & & & & & \\
\hline $\begin{array}{l}1939 \\
1939\end{array}$ & $\begin{array}{l}32 \mathrm{da} \\
32 \mathrm{da}\end{array}$ & $\begin{array}{c}\text { secured } 35 \mathrm{~mm} \text { at } 620 \text { exp. } \\
" 16 \mathrm{~mm} \text { at } 27 \mathrm{ft} .\end{array}$ & & & & & \\
\hline$\frac{C W M I}{1940}$ & 0 da & start W wind & & & & & \\
\hline 1954 & $14 \mathrm{da}$ & start $35 \mathrm{~mm}$ at 620 exp. & $\begin{array}{l}1953 \\
1953\end{array}$ & $\begin{array}{l}16.40 \\
16.40\end{array}$ & $\begin{array}{l}18.40 \\
20.00\end{array}$ & $\begin{array}{l}2.00 \\
3.60\end{array}$ & $\begin{array}{l}\text { bl.gr. } \\
\text { yollow }\end{array}$ \\
\hline 1954 & $14 \mathrm{da}$ & $\begin{array}{l}1 / 10 \text { sec. } 1-10 \\
\text { start } 16 \mathrm{~mm} \text { at } 27 \mathrm{ft} . \\
1-6.3\end{array}$ & & & & & \\
\hline
\end{tabular}


ang

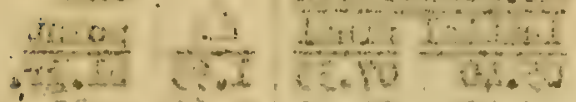

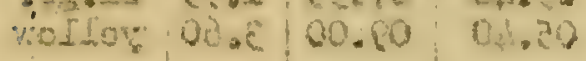

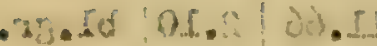
vorison

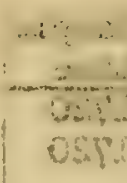

$\cos 1$

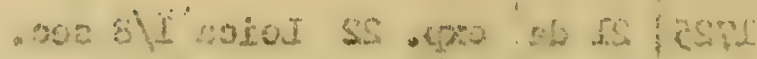

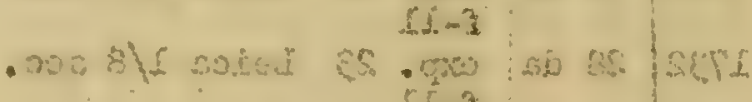

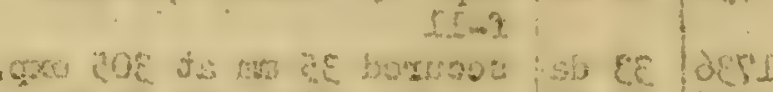

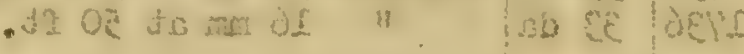

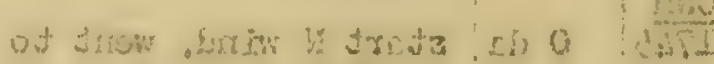
atcho

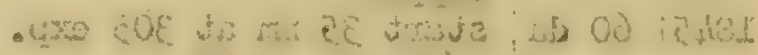
$0 I-1 \cdot 0 . j=0 \Omega$

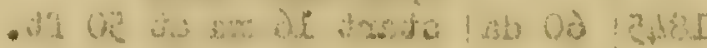

$$
\text { (.3) }-1
$$

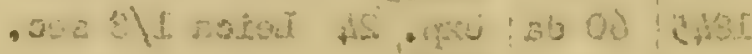

(स)

पर्दू

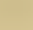

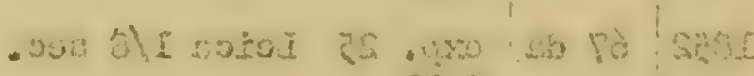
$-i=0$

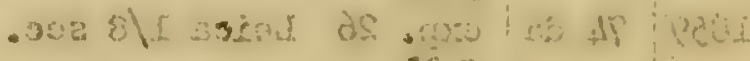

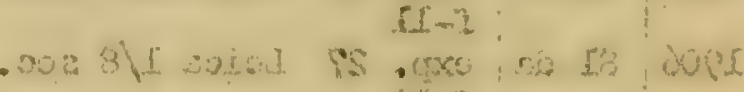

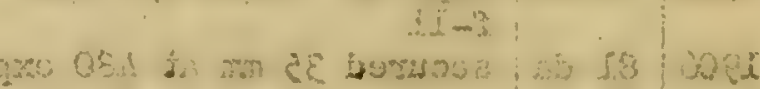

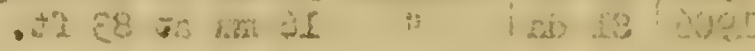

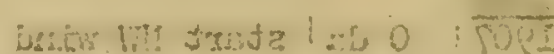

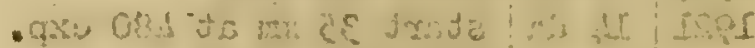
un-i.

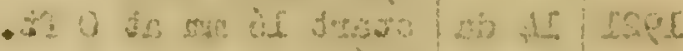
(". ते 2

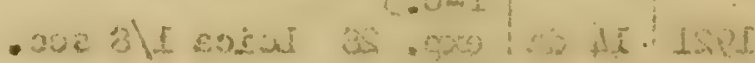

aso $\sin (5)$

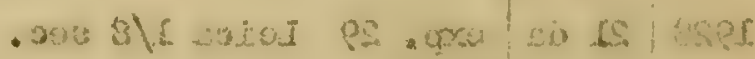

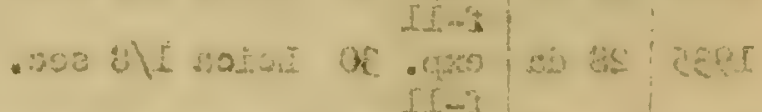

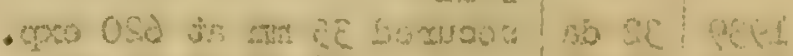

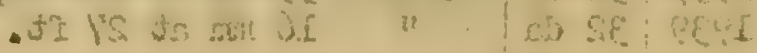

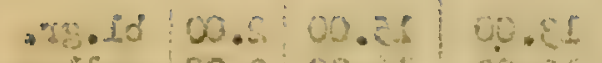

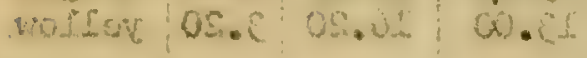

$.07 .500 .5: 03.05$ marber does; $00.0 \mathrm{~g}$
0.1 .01 $0, \hat{1}=1$ $\operatorname{sepr}$ çp?

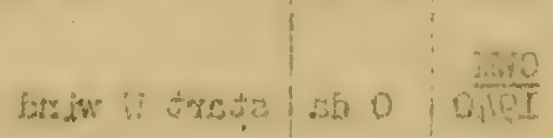

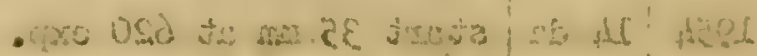
Orm. . 2018 OLIL

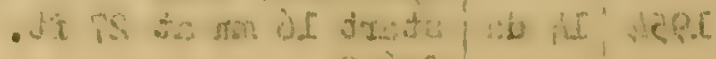




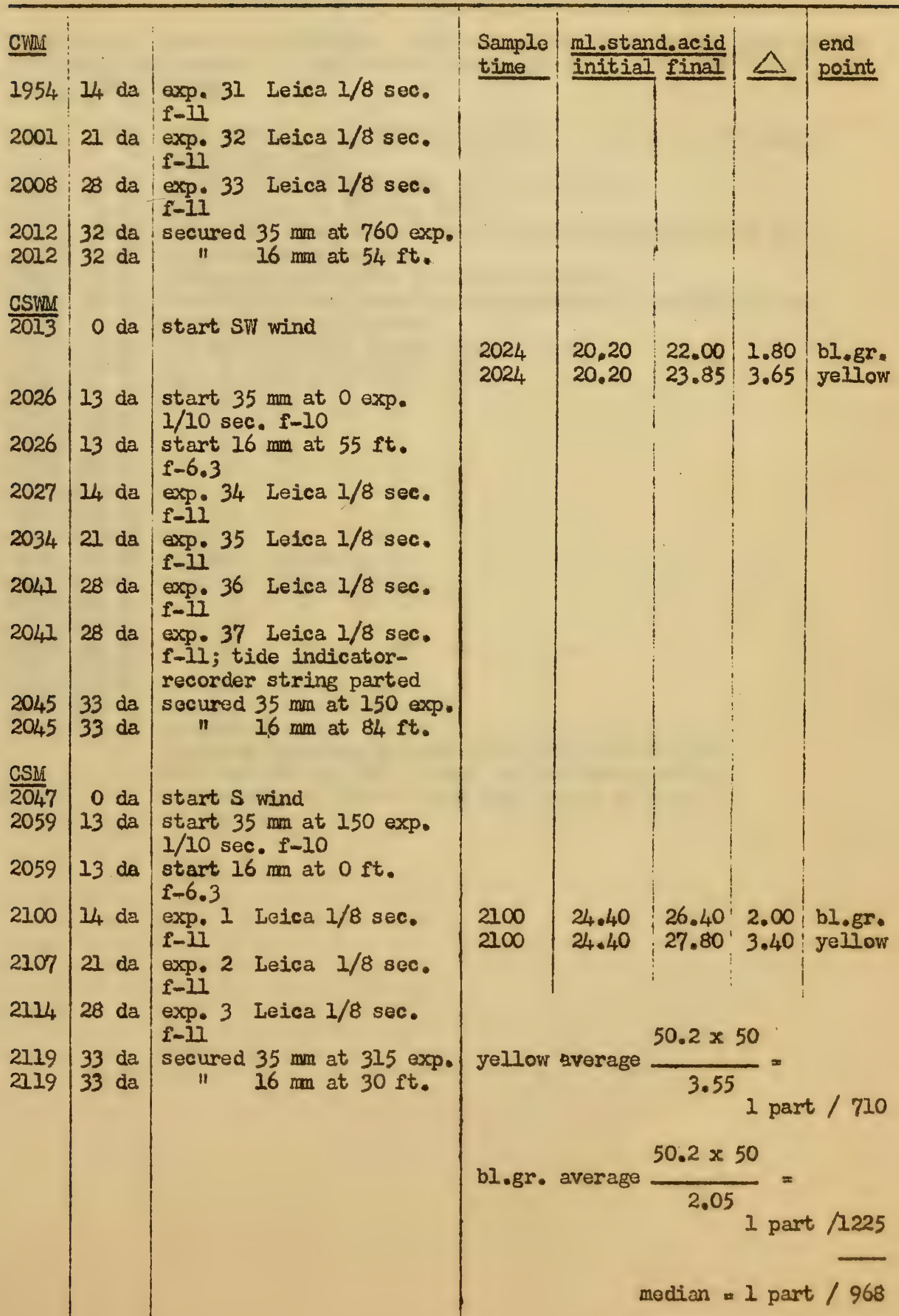


$\therefore \quad \therefore \quad \therefore \quad \therefore \quad \therefore \quad \therefore \square$

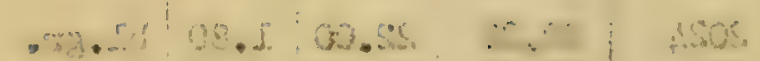

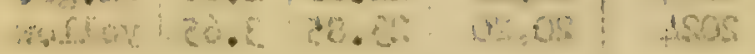

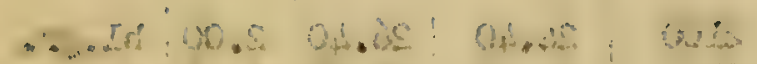

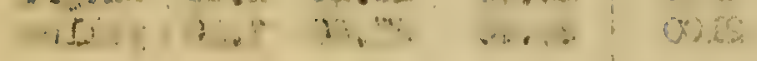
$\because \cdots \cdots+$

\section{$62 \times \therefore .02$}

stosents

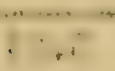

$$
\begin{gathered}
\quad \cdots+\infty \\
\ldots \ldots+\ldots
\end{gathered}
$$

$\therefore \ldots$

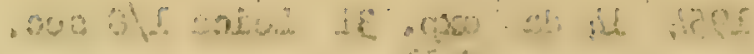

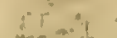

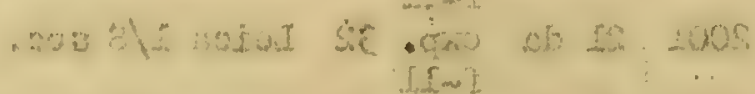

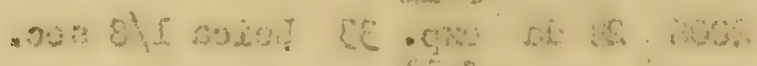
$1+2-1$

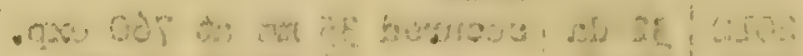

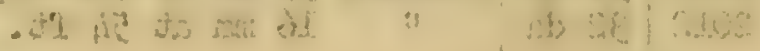

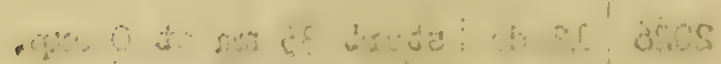

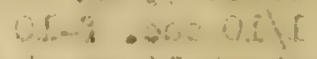

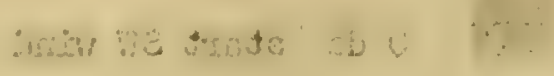

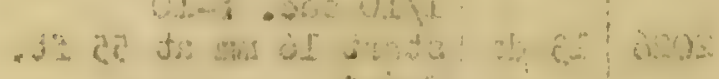

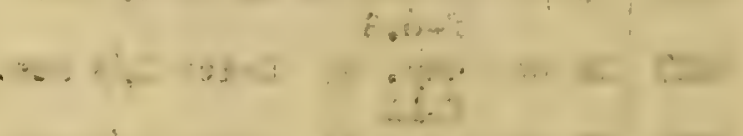

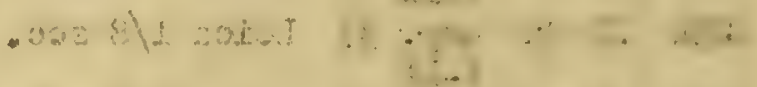

$\begin{array}{llllll}\ldots & \ddots & \cdots & \ddots & \ddots & \therefore\end{array}$

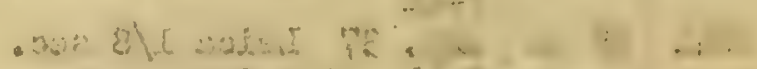

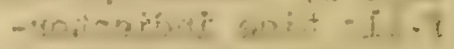

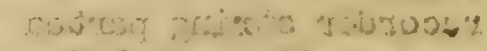

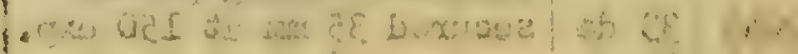

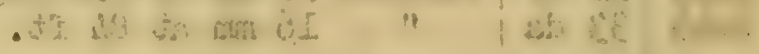

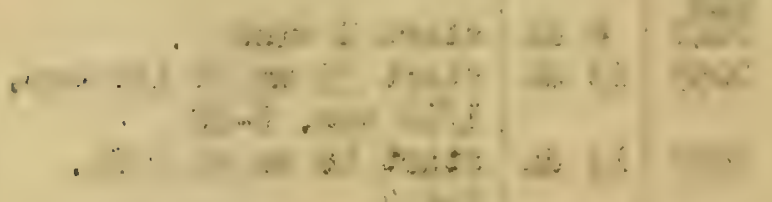

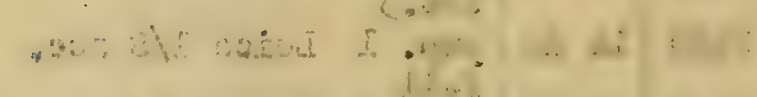
. N14 $\quad \therefore \quad . \cdots+1$

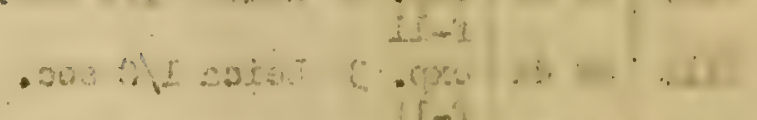
- a je sin 


\section{Octaber 1949 FRESH WATER DISCHARCE}

Progress of fresh water discharge from the northern watershed through the Barataria Bay estuary. Fresh water discharge rate set at $168 \mathrm{cc} / \mathrm{min}$. W $450 \times 10^{6} \mathrm{ft} .3 /$ day.

$1327-5$ da Brom Gresol Green solution added to Little and Mud Irakes equivalent box

13320 da start $35 \mathrm{~mm}$ at 310 exp., $1 / 10$ sec. $f-10$

$13320 \mathrm{da}$-start $16 \mathrm{~mm}$ at $30 \mathrm{ft} ., \mathrm{f}-6.3$

13375 da added more Brom Cresol Green solution to equivalent box

$141745 \mathrm{da}$ reloaded $16 \mathrm{~mm}$ camera.

142250 da changed record rolls on tide recorder

$143564 \mathrm{da}$ added more Brom Cresol Green solution to equivalent bor

$1436 \quad 65$ da reloaded $35 \mathrm{~mm}$ magazine

145785 da dye entered Lake Grande Ecaille area

$1457 \quad 85$ da exp. 4 Leica $1 / 8 \mathrm{sec}$. I-11

$145785 \mathrm{da}$

$150290 \mathrm{da}$

$1.50290 \mathrm{da}$

$150290 \mathrm{da}$

$1513100 \mathrm{da}$

$1513100 \mathrm{da}$

$1513100 \mathrm{da}$

$1524.111 \mathrm{da}$

$1532120 \mathrm{da}$

$1532120 \mathrm{da}$

$1532120 \mathrm{da}$

$1532120 \mathrm{da}$

$1534122 \mathrm{da}$

1534122 da

1534122 da

\begin{tabular}{|c|c|c|c|}
\hline & & " & \\
\hline " & " & $"$ & " \\
\hline " & $"$ & " & $n$ \\
\hline " & $"$ & " & $n$ \\
\hline$"$ & " & " & $"$ \\
\hline$" 10$ & " & " & 18 \\
\hline 11 & " & $n$ & " \\
\hline
\end{tabular}

reloaded 16 mom camera

exp. 12 Leica $1 / 8$ sec. $f-11$

$\begin{array}{llllll}\text { "13 } & 13 & \text { " } & \text { " } & \text { " } \\ \text { " } & 15 & \text { " } & \text { " } & \text { " } & \text { " } \\ \text { " } & 16 & \text { " } & \text { " } & \text { " } & \text { " }\end{array}$

secured $35 \mathrm{~mm}$ at 450 exp.

secured $16 \mathrm{~mm}$ at $15 \mathrm{ft}$.

This run rejected because fresh water discharge rate dropped to $127 \mathrm{cc} / \mathrm{min}$. without being noticed and because of drafts from open doors in waist of building. 


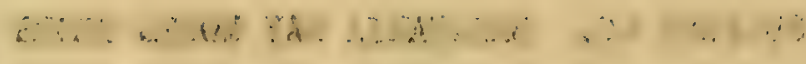

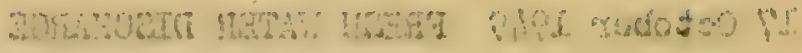

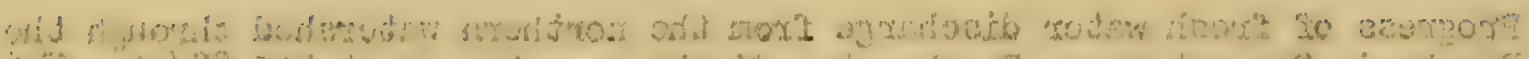

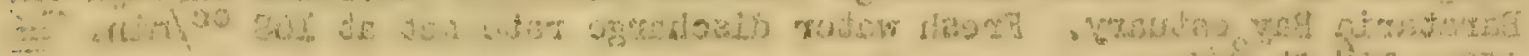
Wat +1 be $x$ act

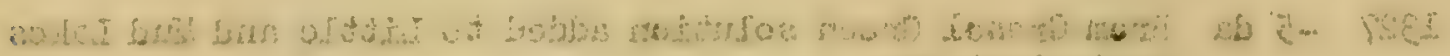

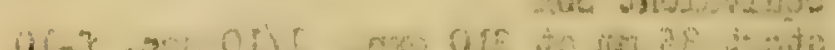

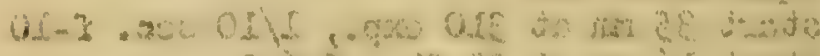

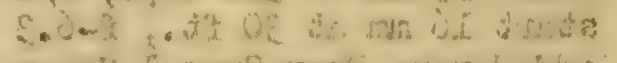

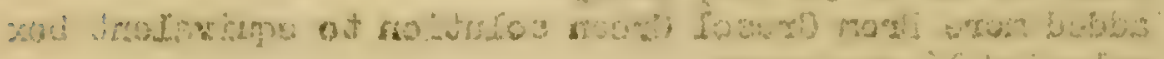

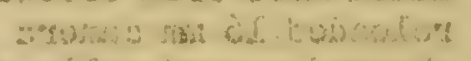

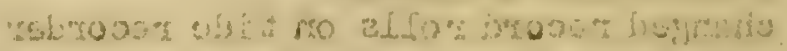

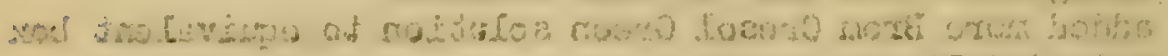

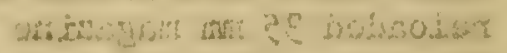

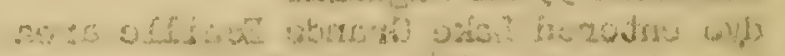

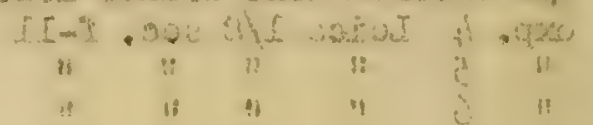

$\therefore 60$

wis 0 क 28

(I) $<$ iefic

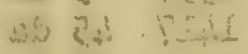

(a) on sich

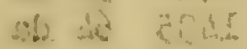

ais cे की

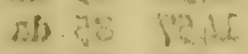

sis to

In 30 sid

ab of sol

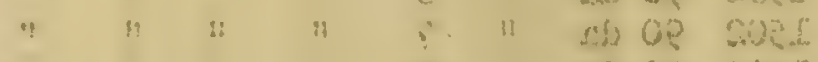

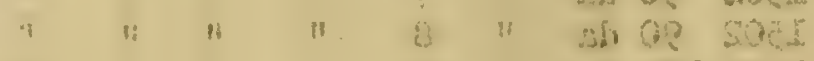

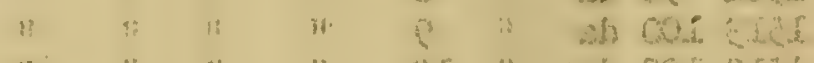

\begin{tabular}{|c|c|c|c|c|c|}
\hline$r:-$ & $\therefore$ & if & sistivit & $\therefore$ &, $4 j+2$ \\
\hline u & 11 & $\because$ & $n$ & CI & $\because$ \\
\hline " & $" 1$ & $n$ & ib & I & $\theta$ \\
\hline if & $" n$ & $n$ & $4:$ & $\therefore 1$ & 12 \\
\hline "i: & $"$ & s: & $\because$ & 6. & it \\
\hline
\end{tabular}

"1 " $"$ " 14 "

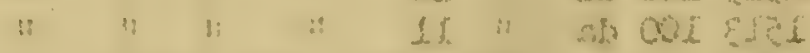

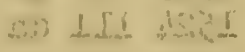

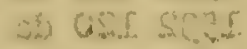

$\because$ and Sict

$\therefore \cos \operatorname{sen} 2$

ald $05 \mathrm{sic}$

an. OF

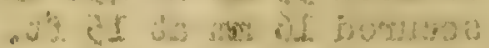

$\because 3$ SS: is d

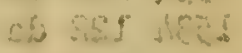

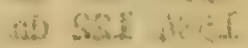

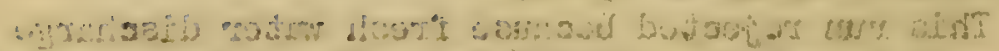

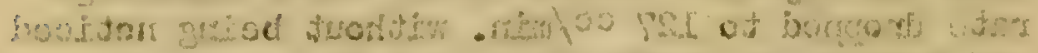

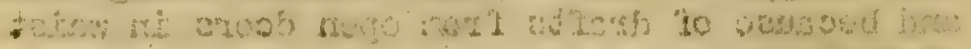

$$
\therefore \therefore \cdots
$$


18 October 1949 TEST AO

Progress of fresh water discharge from the northern watershed through the Barataria Bay estuary.

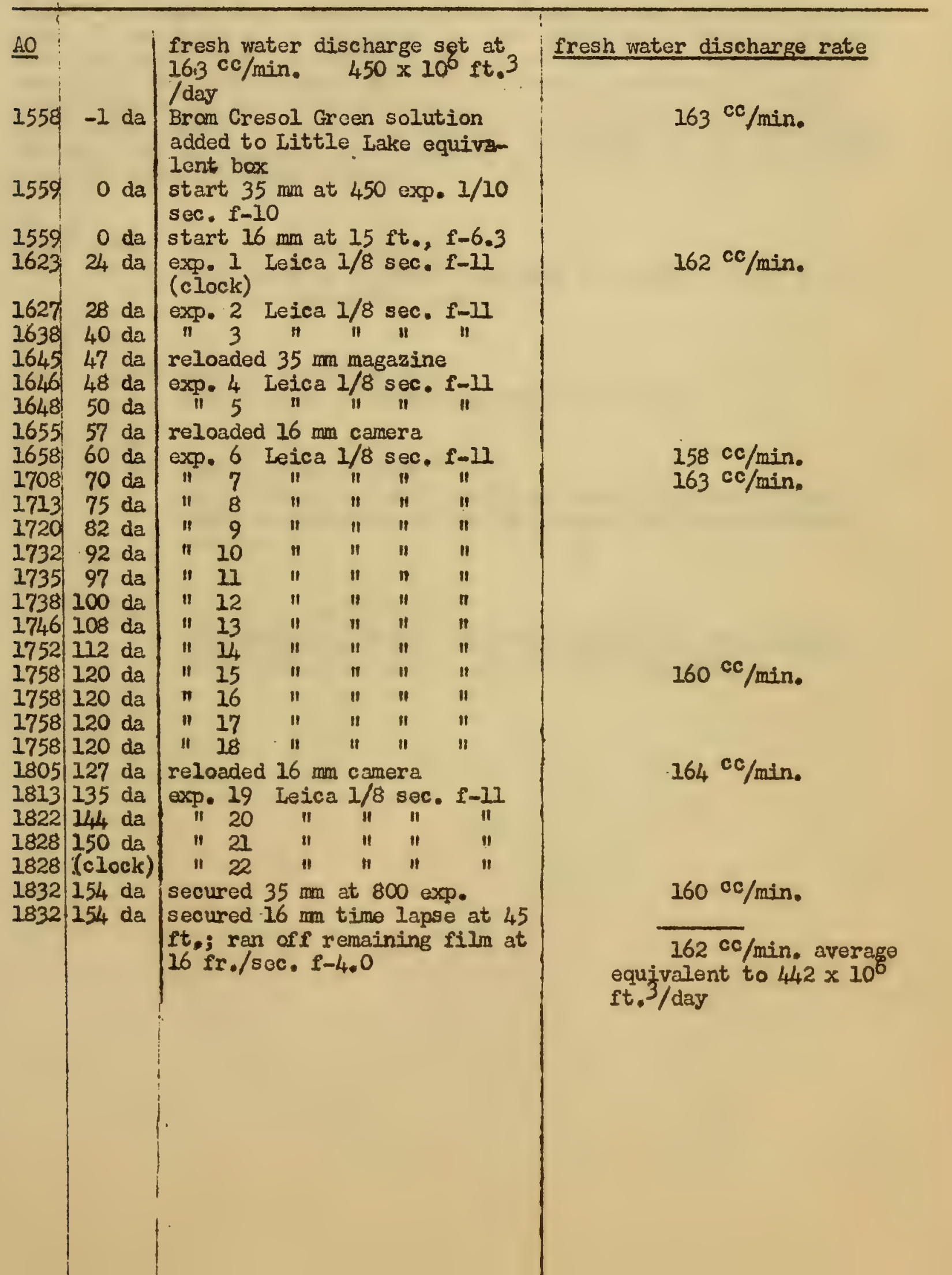




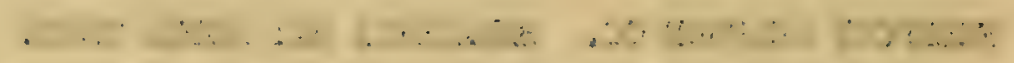

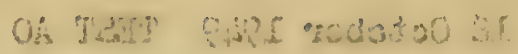

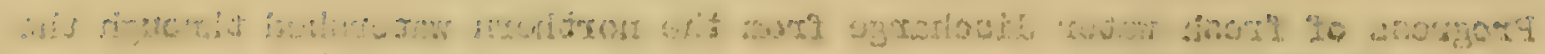

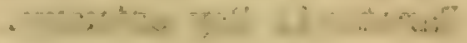

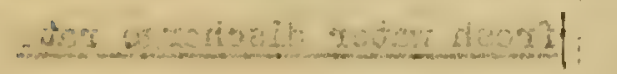

asis: 230 is

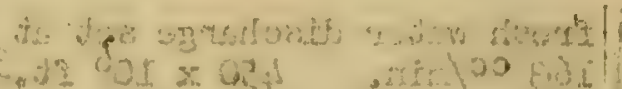

1.

van \)

(a)

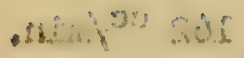

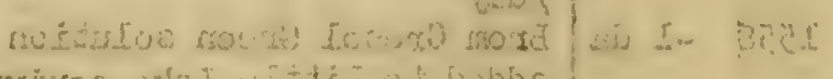

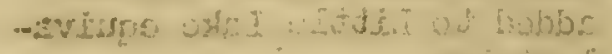

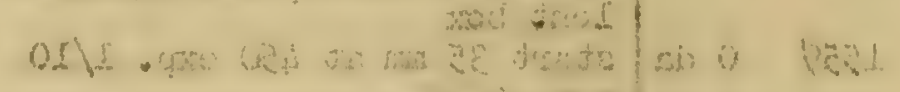

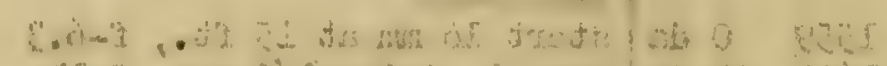

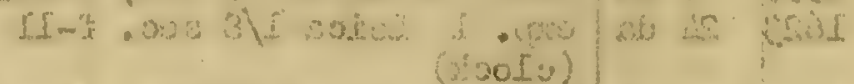

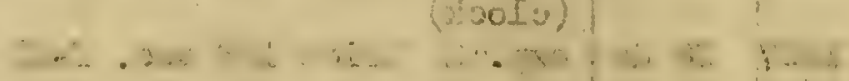

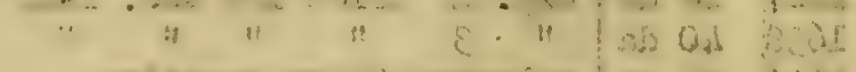

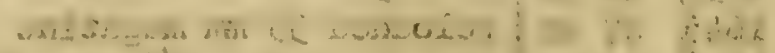

I.

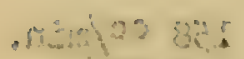

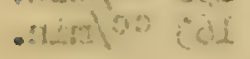

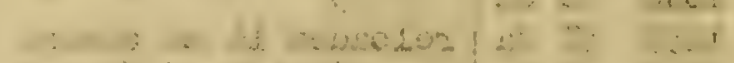

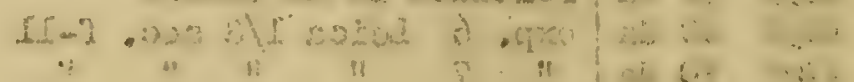

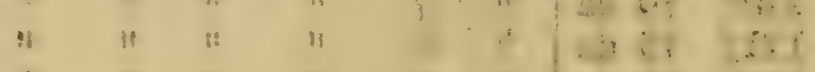

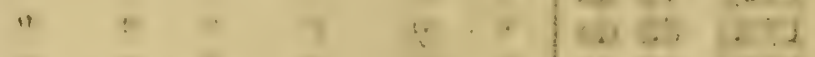

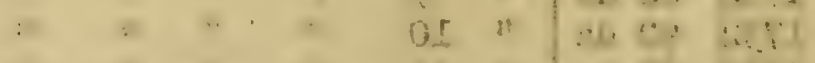

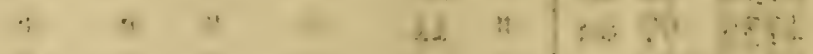

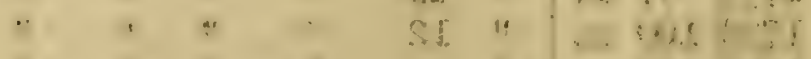

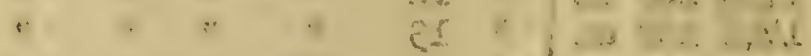

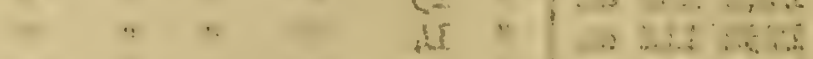

- Himlis od

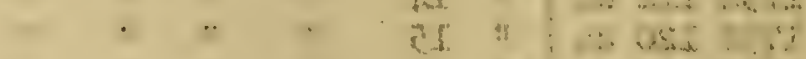

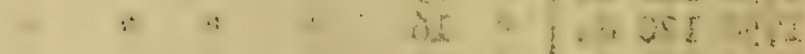

$\therefore$ " 11 "1

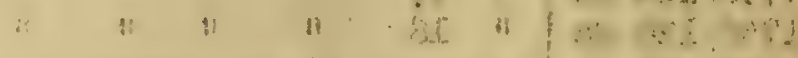

orisitis tist

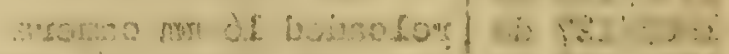

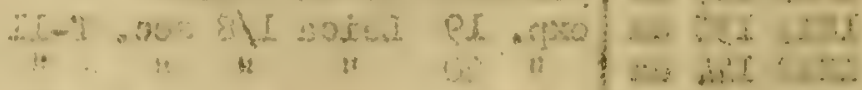

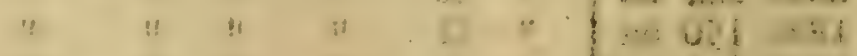

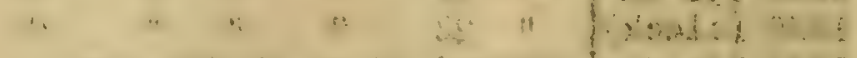

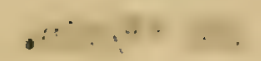

abvo .

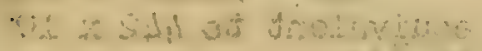

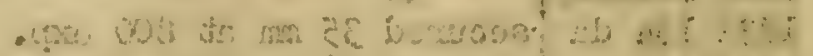

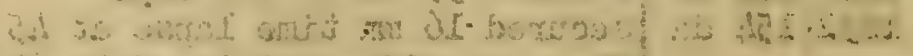

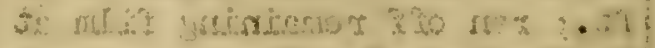




\section{October 1949 TRAVEL TIME TESTS ON EBB TIDE FROM POINT VI}

An attempt was made to discharge Carter's midnight black ink for I model "hour" ( 2.5 sec.) from Point VI on Humble Canal at the beginning of slack ebb. It was hoped that such a dye in clear water woulu cohere enough to give some measure of the earliest arrival of bleed water in northern Bay Rouquille. The ink solution was not sufficiently coherent to represent parcel motion but only that of a certain finite dilution, hence these experiments reveal axrivals at a dilution that is very great but unknown. Discharge pulses liberated from Point VI at slack ebb during maximum symmetrical tides of tropic sequence.

VI $A I$

1032 o hr exp. 17 Leica $1 / 8 \mathrm{f}-11$

exp. $18-36$ at 3 "hour" intervals thereafter, 1.e., every 7.5 sec. mean solar time

${ }_{1045}{ }^{A_{2}} 0 \mathrm{hr}$ exp. I Leica $1 / 8 \mathrm{f}-11$

exp. $2-20$ at 3 "hour" intervals thereafter

VI ${ }^{A}{ }^{\mathrm{T}}{ }^{3}$ o hr exp. I Leica $1 / 8 \quad 1-11$

exp. 2-20 at 3 "hour" intervals thereafter; same faint color reached west end of Lake Grand Ecailize on surface (tension?)

$\operatorname{VII}_{1725^{\mathrm{A}}}{ }_{4}^{\mathrm{T}}$ O hr exp. 1 Leica $1 / 8$ f- 11

exp, 2-20 at 3 "hour" intervals thereafter; some surface film again reached west end of Lake Grand Ecaille 


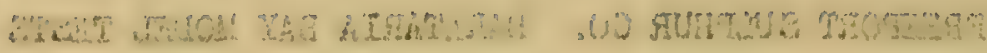

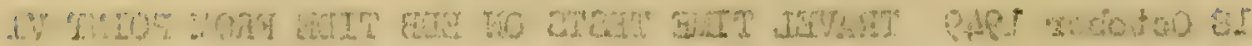

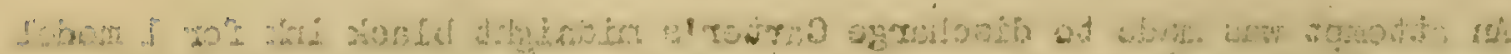

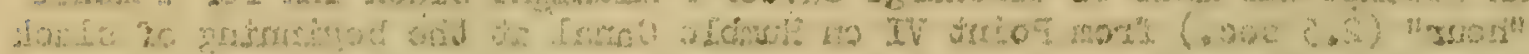

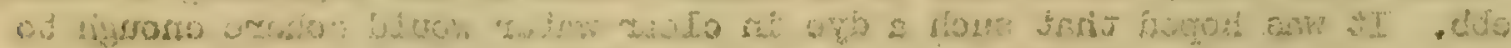

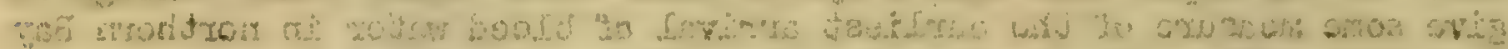

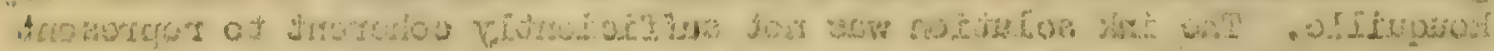

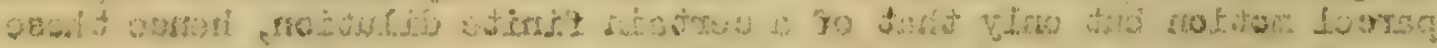

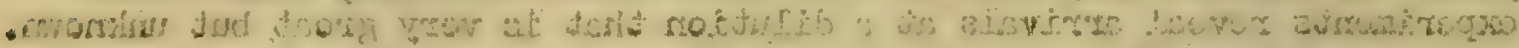
Hive mantion

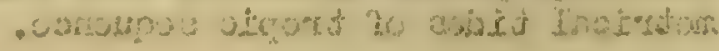

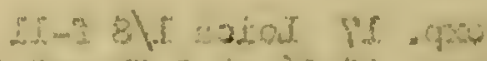

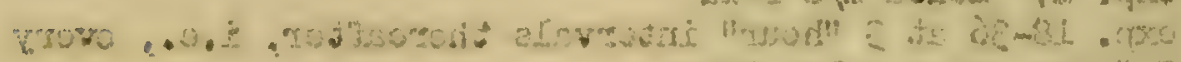
क्या广

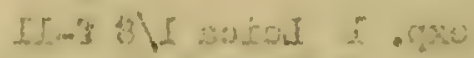

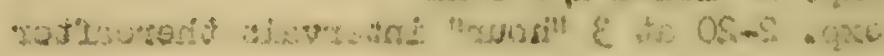

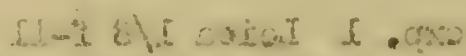

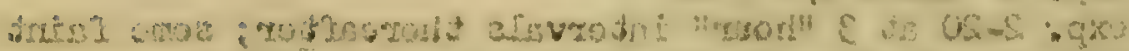

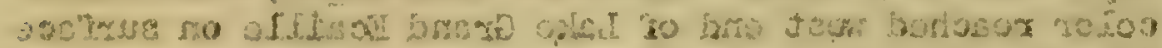

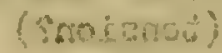

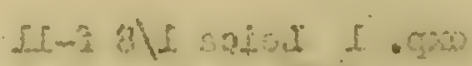

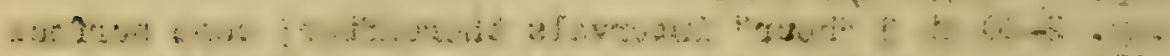

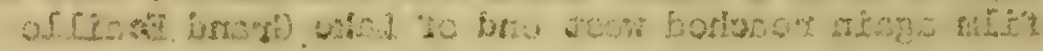

if

xis I. IV IV $\operatorname{sic} 015$

xid c) ciol

anto 0 MOLE 


The following pages are photographs of a series of 41 drawings representing the continuous and intermittent distribution of simulated bleed water observed in the Barataria Bay model during the condition $\mathrm{B}$ and $\mathrm{C}$ model tests. The boundaries of the areas were obtained from 3cparate examination of each of the approximately 13,000 double $35 \mathrm{~mm}$ frames on the films exposed in the Graflex Photorecord camera. This camera recorded the distribution of simulated bleed water 8 times every model "day," that is, every 3 model "hours" throughout the model tests. The examination was performed by projection of the film strips on base charts prepared from a rubbing of the model. Each successive projected image was made first to coincide with the base chart and then the areas showing the yellow or green color of unneutralized acid were shaded in pencil and the boundaries drawn as a sharp line. The envelopes of all the outermost boundary lines of the several tests and their sumaries are presented in the finished drawings that follow.

1. Summary of the distribution of color in all $M$ and CC tests.

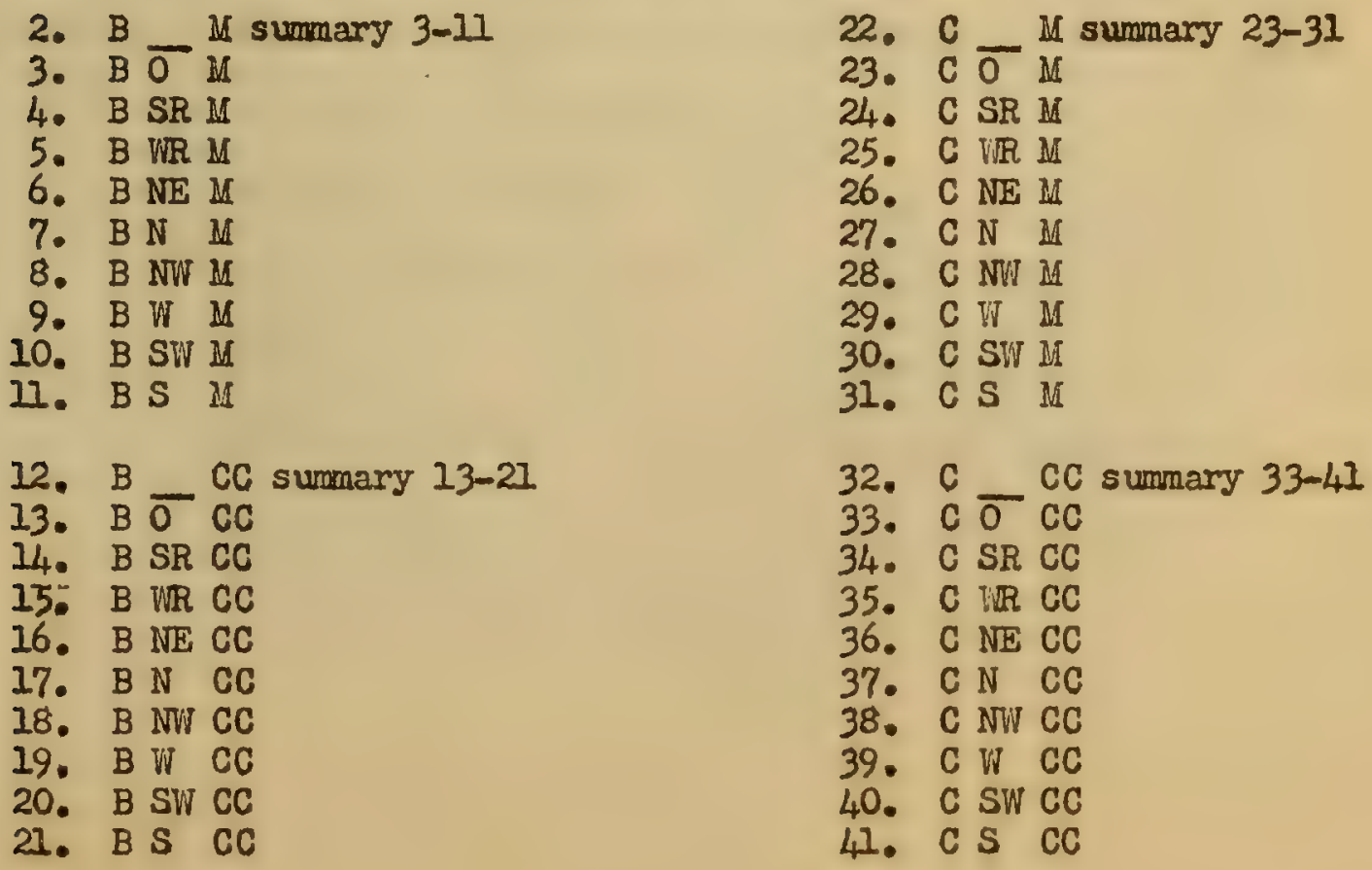




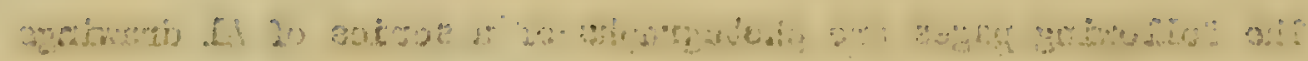

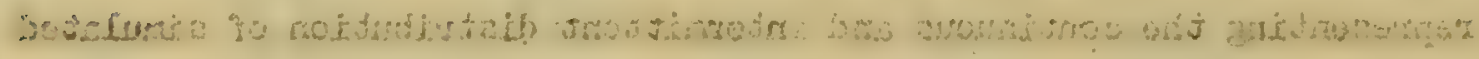

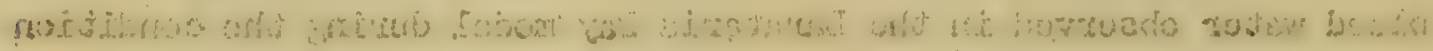

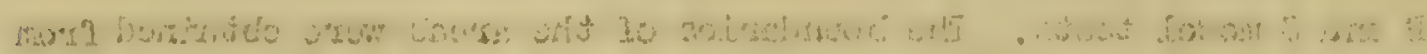

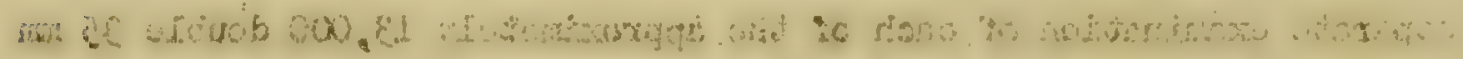

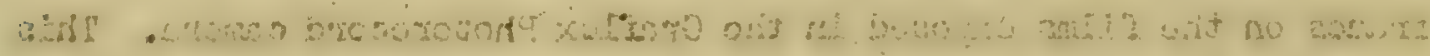

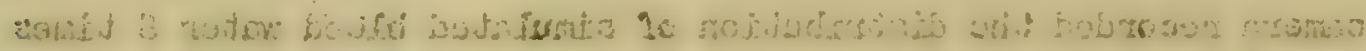

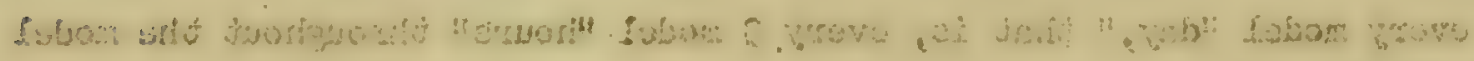

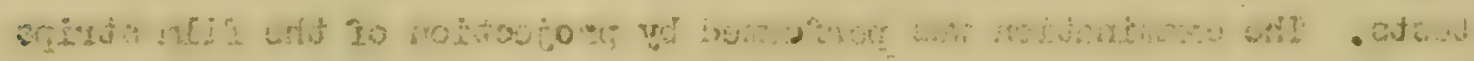

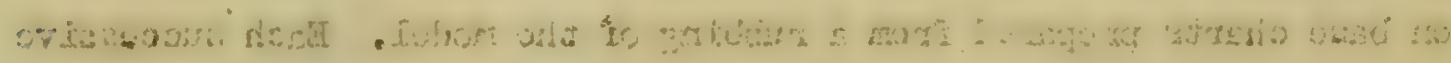

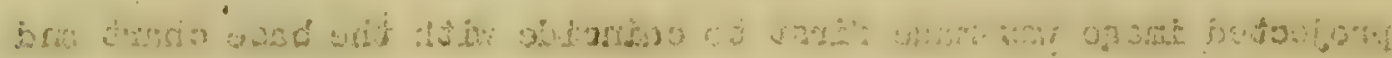

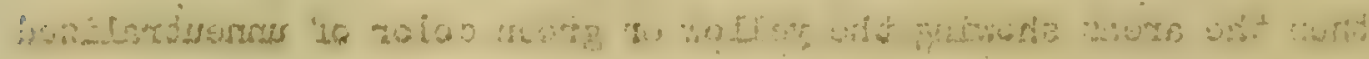

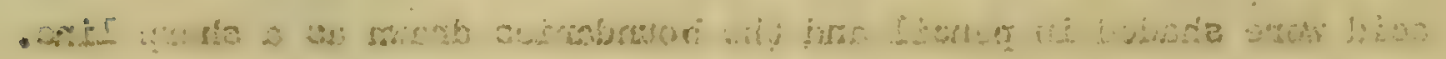
-

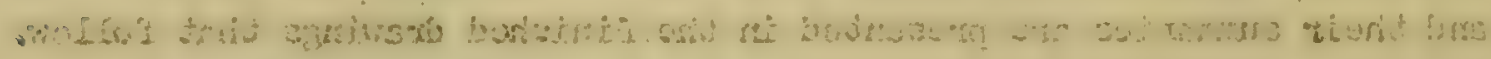

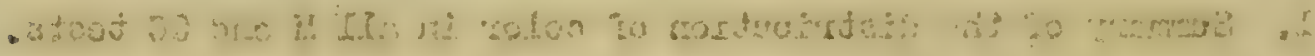
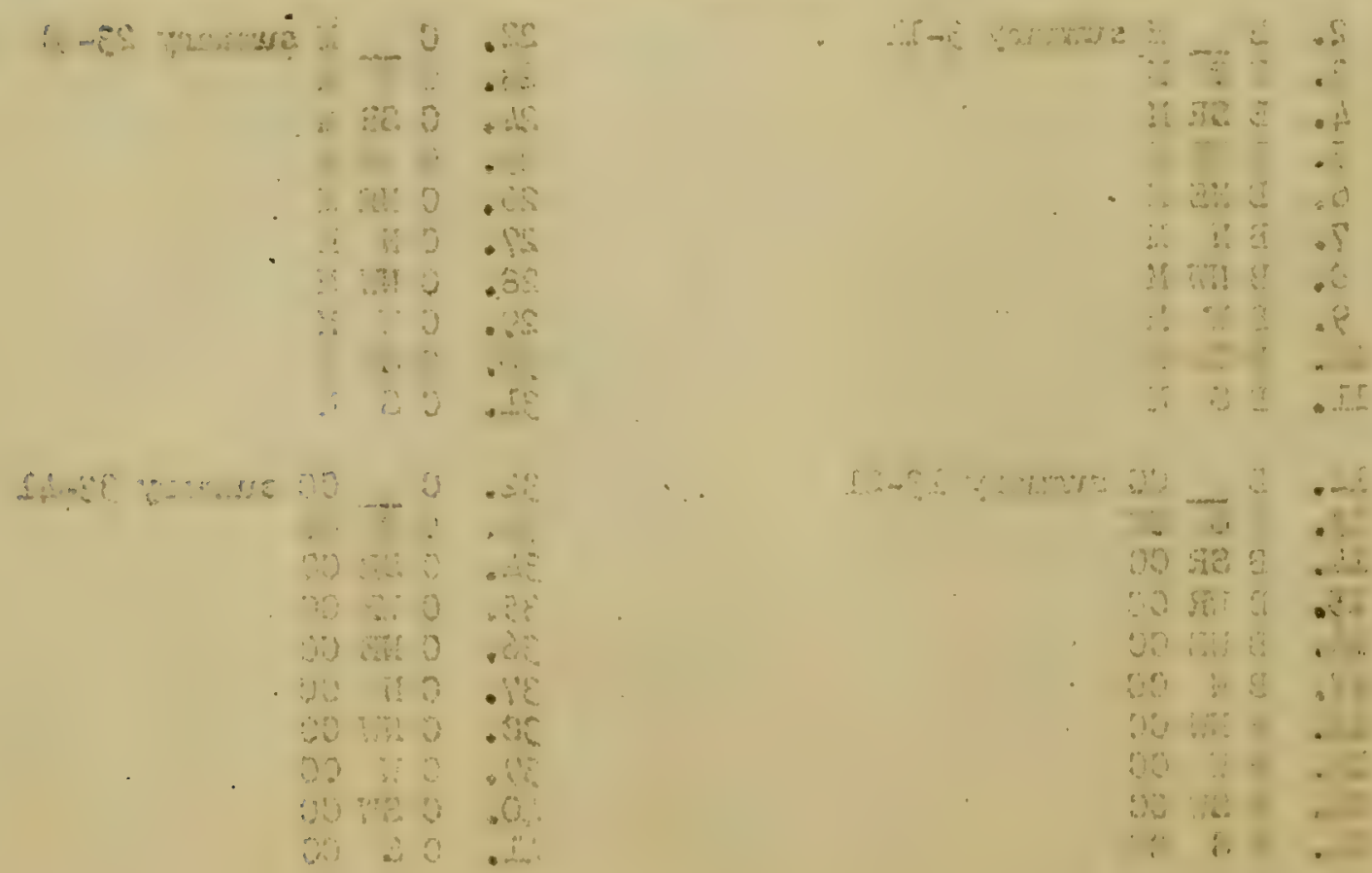
Contents

I. Sumnary - Introduction I

II. The Role of Modern Hydraulic Models 9

III. The Scales of the Barataria Bay Model 11

IV. Reconciliation of Kinematic and Dynamic Processes 16

V. The Tide Machine 24

VI. Kinematic Verification of the Model 29

VII. Specifications of the ModeI Tests 32

VIII. Chemistry of the Bleed Water Tracer 34

IX. Auxiliary Pumping Systems 39

X. The Reliability of the Model Tests 43

XI. "First Arrival" Tests 44

Appendix I Schedule of procedure in model tests

II Photographic and Chemical log

III Drawings of results of model tests

IV Tide records attached to Copy No. 1. The tides are also indicated on the time-lapse motion and still pictures of the model tests

V U. S. Weather Bureau reports from Grand Isle, Iouisiana station attached to Copy No. I 



\section{SUMMARY - INTRODUCTION}

In late December 1948, the Freeport Sulphur Company requested the writer to undertake construction of an hydraulic model of the Barataria Bay area in which their Lake Grande Ecaille, Louisiana, mine is located (see frontispiece adapted from Chart 1273 U.S.C.\& G.S.). In this area, roughly 25 miles square, the characteristic depth of water is about three feet, necessitating either a very large model or a highly distorted smaller model. It was decided to make the model eight feet square, and employ a horizontal scale of $1 / 20,000$ and a vertical scale of $1 / 192$; that is, employ a vertical exaggeration of $1 / 104$. The model was built and operated on the property of the Woods Hole Oceanographic Institution in Woods Hole, Massachusetts.

The model was constructed on a strong bed of $2 \times 12$-inch fir timbers (fig. 1) arranged to form a tight network of triangular voids. The framework was then covered with $3 / 4$ inch marine plywood in steps (fig. 2) so cut that the treads had the shape of the intervals between successive 12-foot contours on the bottom topography in the prototype (fig. 3). This "staircase" was boxed in by a heavy combing, caulked, (fig. 4) and covered with a lath of hardware cloth (fig. 5). Over this a 4-inch thickness ( $3 / 4$ ton) of Hydrocal Bull (precision pattern-maker's $\mathrm{CaSO}_{4}$ plaster) was cut as a monolith in which the intricate modeling of the bays and islands could be cut with rotary tools. The layout of the land and islands 

was projected from the top of a tower (fig. 6) at a height of 16 feet above the model surface, traced off, (fig. 7), and routed in relief (fig. 8) so that sea level lay $1 / 4$ inch below the land surface. The soundings were then entered on the sea level surface from the same lantern slide and projector, and additional soundings were entered from the original data sheets provided by the U. S. Coast \& Geodetic Survey. Soundings were realized by routing between carefully drawn contours ( $f$ ig. 9). The steps were then blended with hand tools (fig, 10). The resulting surface (fig. 1I) is probably accurate to \pm 0.015 inch ( \pm 0.25 "foot") vertically and \pm 0.03 inch ( \pm 50 "feet") horizontally. Sea level was established as a level plane ( \pm .005 -inch) by means of repeated checks with a precise transit on the pair of rails from which the routing tools were suspended.

Gravitational (Froude) scaling of the kinematic ratios of time, velocity and discharge was used unmodified except at the passes connecting the bays with the Gulf of Mexico. In each of the passes the velocity and the turbulence (measured in terms of eddy diffusion) of flow were initially too high. In order to correct this common situation, roughness was artificially increased in the passes by means of wire screens ( $20 \times 20$ mesh). Increased roughness in these critical channels not only decreased the velocities so as to agree with the Froude scales but corrected to an acceptable degree the characteristics of channel turbulence as well. To verify the over-all kinematic behavior of 

the flooded model, as many hydraulic occurrences as possible were compared with data from the detailed field reports of H. A. Marmer, and observations by V. H. Brogdon and the writer. With few adjustments of the model the times of slack water, the occurrence of high and low water, the particle excursions, and magnitudes of velocities were reproduced at a large number of points. Auxiliary equipment was designed to reproduce tides, winds, rainfall and industrial discharge scaled in accordance with the model constants.

The tide machine was composed of three drums, each of which displaced water and changed sinusoidally the water lovel in the model at rates in accordance with the time scale $1 / 1440$ (one minute per model "day"). Each drum was driven by a scotch yoke through a stroke of 2 inches; the semi-diurnal drum having a displacement determined by the sum of the amplitudes of the $\mathbb{N}_{2}, S_{2}$ and $\mathrm{N}_{2}$ tidal components and a speed number of the average of the component speed numbers, the first diurnal drum a displacement determined by the amplitude of the $O_{1}$ tidal component and a speed number of the $O_{1}$ component, and the second diurnal drum a displacement proportional to the sum of the $K_{1}$ and $P_{1}$ tidal components and driven at the

I/ The Tide in Barataria Bay, U. S. Coast \& Geodetic Survey, Washington, D. C., July 1947.

The Currents in Barataria Bay, U. S. Coast \& Geodetic Survey, Washington, D. C., February 1948. 

average speed of these two which is precisely a mean solar day per $360^{\circ}$. Thus, the second diurnal drum was run at one cycle per minute and the other two at 30.5 seconds and 64.5 seconds per cycle respectively. This combination produced a succession of great diurnal tides yielding to lesser semi-diurnal tides at intervals of about two "weeks," a progression characteristic of this region of the Gulf of Mexico (fig. 12).

The winds were generated by tank-type vacuum cleaner blowers mounted around the model at the cardinal and intercardinal points. Each was wired to a switch at a central control point and its rate of pumping governed by a Variac. Thus, it was possible to vary the wind speed and direction to reproduce either the detailed or average wind as it occurred during the periods of interest to the sponsors and in accordance with the records of the U. S. Weather Bureau reports from Grand Isle, Louisiana. Velocity calibration of the wind machines was accomplished by observing the rate at wich the surface layer of water in the model was propelled under wind stress at the steady state. It was assumed on the basis of field experience and values derived from empirical equations for wind stress that in more than 10 miles of fetch the stress on the surface is such as to produce an equilibrium surface current speed between 2 to $3 \%$ of the wind speed. By this

2) See Appendix $V$ attached to Copy No. I.

3/ Sverdrup, Johnson \& Fleming, The Oceans, Prentice Hall, New York, 1942, pp. 489-503. 

measure, it was possible to produce winds of hurricane force (100 "knots") from the simultaneous draught of three blowers running at full speed. Due to the small horizontal scale and the vertical exaggeration of the model, the capillary wave height was too small and the capillary wave length was too large for wave action to be in scale or to produce the significant mixing effects found in nature.

Rainfall, which amounts to 60 inches per year on a watershed of some 1100 square miles to the north of the modeled area, drains through the modeled area at the rate of approximately $450,000,000$ cubic feet per day, according to Marmer. This flow was introduced in the Little Lake equivalent box at the northwest end of the model by means of a pump attached to the tide machine. This pump drew water from the Gulf and produced both a closed circulation system for the rainfall, and a current of water through a venturi which generated a slow longshore current moving westward along the coast in agreement with known offshore circulation.

Industrial wastes (also referred to as bleed water) were introduced into the model through copper tubes embedded in the plaster and terminated at their proper geographical positions on the Freeport Sulphur Company properties. The injection ends of the tubes were attached to open glass cups at the level of the water in the model.

4/ The rainfall on the modeled area was disregarded. 

Each cup was supplied at first by a calibrated dropper and later on by the outlet of a proportioning funnel, both of which in tum being fed by a $100 \mathrm{cc}$ hypodermic syringe acting as a positive displacement pump closed by a motor-driven screw. Thus, it was possible to know and control the quantities of industrial waste being introduced into the model either by counting drops delivered per minute from calibrated dropper tips or by timing the revolutions of the motor-driven screw. The industrial waste was simulated by an acid. The acid concentration chosen was such that when it mixed with a given number of volumes of ambient model water, containing an indicator solution of brom cresol green, it changed color from yellow to blue. The model water was set chemically to a known alkaline state (blue) such that for a given acid strength the color change (yellow-blue) would occur at a predetermined dilution. In this way the industrial waste, more concentrated than any specified dilution figure was represented by yellow water, and further dilutions in excess of those specified by a pleasing blue color which pervaded the whole model and varied in intensity with the depth of water.

Under certain conditions, continued discharge of industrial waste eventually extended unneutralized acid boundaries into the Gulf portion of the model. If routine titration of samples of water drawn from the Gulf showed it necessary, as the unneutralized acid reached and mixed away in the Gulf, a base of identical strength was introduced volume for volume into the Gulf at the 

circulating venturi. In this way the ambient water in the Gulf volume was rendered chemically infinite and was maintained quantitatively in its alkaline condition. Thus the model could be run indefinitely to study the steady states of waste distribution in the bays under a variety of controlled canditions.

The results of the model studies were recorded automatically in color photographs made simultaneously with each of two cameras (1) a $35 \mathrm{~mm}$ Graflex pneumatic microfilming camera operated once every 7.5 seconds producing 8 exposures per model "day" and (2) a $16 \mathrm{~mm}$ Bolex time-lapse motion-picture camera operated electrically at 1 exposure every second yielding 60 exposures per model "day" and effecting a contraction of time, when projected at 16 frames per second, of $1 / 23040$, that is, of 1 model "day" per 3.75 mean solar seconds. Light for photography was provided by 12 No. 2 photoflood lamps in reflectors, a total of 6000 watts illumination in controllable quantities for uniform lighting. An electric clock fitted with a dial graduated in 24 model "hours" swept by the second hand, 60 "days" indicated by the minute hand, and 24 "months" indicated by the hour hand, was mounted in the field of view of both cameras so that the elapsed time from the beginning of each experiment was automatically indicated and totalized. Similarly the contribution of each of the tide drums was integrated and the sum of their effects indicated by a traveling pointer on a scale large enough for the cameras to record the height of water to 0.05 scale "foot." The same integrator was coupled to a pen 

which wrote a record of the tides on a paper tape moving at $1 / 2$ inch per minute ( $\mathrm{fig} .12$, and completely represented in Appendix IV of Copy No. 1 only). The wind direction was indicated by a small banner on a circular card placed on an island (unnamed) near the center of the model. In "no wind" conditions this indicator was removed from the model.

The tests of the model consumed approximately 65,000 model "hours" (about seven and one-half model "years") and were recorded in color on approximately 13,000 frames of $35 \mathrm{~mm}$ film strip and 3,000 feet of $16 \mathrm{~mm}$ time-lapse motion-picture film. The bulk of this record has been reduced to a series of 41 sumary drawings (Appendix III) representing the continuous and intermittent (tidal) excursion of the simulated industrial waste in the model for each wind condition in each of two major bleed water discharge conditions, Tests $\mathrm{B}$ and $\mathrm{C}$, and two concentration conditions, nominally $1: 1000$ and 1:200. Test A, waich shows the distribution of fresh water drainage through the model area, is most easily seen in the original $16 \mathrm{~mm}$ time-lapse motion picture. However, figure 13 shows a characteristic stage after 30 "days" advance of fresh water into Barataria Bay from the north. This circulation of fresh water drainage undoubtedly tends to block the otherwise free circulation of water from Lake Grande Ecaille into Barataria Bay. 



\section{THE RDIE OF WODERN HYDFAULIC MODELS}

Hydraulic models as they are understood and employed today are the product of several decades of experimental development. There are no comprehensive mathematical relationships to express the entire range of correspondence between models and their prototypes. Lacking these, the design and operation of hydraulic models is an art and the results obtained are necessarily subject to interpretation. The process of interpretation is frequently qualitative or categorical in the "is" or "is not" sense since a full accounting of all the physical properties of a fluid system is not always possible. Within these limitations, however, the use of models has great interpretive value. Today, most important problems in fluid engineering are solved through combined consideration of model experiments and mathematical analys is of the known physical factors.

The function of a model is essentially that of an analogue computer. When a geometrically extensive or lengthy series of hydraulic events involves a great number of complex factors it is too difficult for the human mind and too laborious for exact mathematical analysis to cope with them all simultaneously. Usually, more intelligible results can be obtained if a similar system is constmucted and studied under controlled laboratory conditions.

The degree of similarity between a model and its prototype 

may be only geometrical, or it may be kinematic, or dynamic. Two structures are said to be geometrically similar if the ratios of all homologous dimensions are equal. Kinematic similarity is similarity of motion. The motions of kinematically similar systems are geometrically similar and the ratios of the velocities of the several homologous particles in motion are equal. Few models are similar to a higher degree. It is possible, however, for two systems to possess dynamic similarity either in certain or all respects. In order to achieve dynamic similarity the two systems must be kinematically similar and in addition the ratios of the homologous masses and forces concerned in the motion must be equal. The condition of complete dynamic similarity is seldom achieved except in instances where the model is identical with the prototype in every respect. 



\section{THE SCALES OF THE BARATARIA BAY MODEL}

The scales of the Barataria Bay model are based primarily on the equations of kinematic similarity due to Froude. These equations concern the relationships between gravitationally energized fluid systems in a state of continuous quasi-hydrostatic equilibrium. In an estuary like Barataria Bay there are two dominantly gravitational factors controlling the circulation; the tides and the drainage of rainfall. Wind stress is also present and scaled to the model since it produces very significant water motions, but since this force is not gravitational its scaling transcends kinematic relationships.

Once the similarity of circulation in the main bodies of water is established in a model it is possible to introduce an indicator to trace the progress of suitably scaled quantities of industrial discharge under a variety of simulated natural conditions. This was the purpose for which the Barataria Bay model was built.

The notation used in scaling relationships is similar to ordinary physical nctation except that all symbols that apply to the prototype are followed by a subscript "p" and those applying to the model are followed by a subscript "m." Scaling ratios are usually dimensionless and followed by a subscript "r" defined for any quantity $\mathrm{J}$ as 



$$
J_{r}=\frac{J_{m}}{J_{p}} .
$$

It is possible to assign $J_{m}$ unit value and express $J_{p}$ as its multiple. Thus the time scale in a model compressing the events of one mean solar day within the compass of one mean solar minute; $T_{r}=T_{m} / T_{p}=1 / 1440$. The units of the ratio cancel mathematically but if they are preserved the scaling ratio expresses, instance for instance, the proportion in common units of the homologous quantity in either the model or the prototype. In the case illustrated one may justly speak and think interpretatively of a model "day" but this interval is still only one mean solar minute for purposes of calculation.

Choice of the vertical and horizontal scales was determined for the Barataria Bay model by the dimensions of the available space, the vertical distortion required to maintain a useful depth of water and by the rate of going of the model. Because of the complexity of the water-land areas in the Barataria Bay region it was considered imprudent to build the model on a horizontal scale smaller than $1 / 20,000$. This horizontal scale allows all the area of interest to the sponsors and the additional areas containing significant influences of oceanographic and hydrologic kinds, except part of Little Lake, to be included in a space eight by eight feet. The remainder of Little Lake was represent ed by a box of equivalent area mounted on the outside of the model at 

the northwest corner and connected to the fluid system by a tunnel of suitable dimensions. The horizontal scale being thus fixed, and considering the desirability of a time scale of one mean solar minute per mean solar day, the vertical scale was calculated from the kinematic formula of Froude for the distorted open channel model in which the value of $g$ and properties of water are substantially the same for the model and the prototype.

$$
y_{r}=\left(\frac{I_{r}}{T_{r}}\right)^{2}=\frac{1}{193}
$$

This vertical scale is within half of one per cent of $1 / 16$ inch per "foot" and was so adopted. The resulting vertical distortion is

$$
D_{v}=\frac{I_{r}}{y_{r}}=\frac{192}{20,000}=\frac{I}{104}
$$

This amount of vertical distortion is acceptable.

The Froude velocity scale is derived from consideration of the rate of progress of a gravity wave of very great length compared with the depth of wer; that is of tidal dimensions. The rate of progress of such a wave is given by $\sqrt{\mathrm{g} d}$, where $g$ is the acceleration of gravity and $d$ is the depth of water. The acceleration of gravity is substantially the same in both the model and prototype hence the velocity ratio is entirely controlled by the 

square root of the vertical scale, $\sqrt{y_{r}}$. Thus

$$
v_{r}=\sqrt{y_{r}}=1 / 13.9
$$

This means that a velocity of 1 knot in the prototype is represented in the model by a velocity of $1 / 13.9 \mathrm{knot}$ or $3.65 \mathrm{~cm} / \mathrm{sec}$. Since the available data on the velocities of flow in the prototype were given in knots, a ruler was constructed and graduated in $3.65 \mathrm{~cm}$ intervals with tenths on one side and quarters on the other so that the velocities in the model might be checked with these lengths against the seconds beat of a break-circuit chronometer made audible through a loud speaker.

With the establishment of the time and length scales it was possible to calculate the discharge scale for the rainwater and bleed water. The kinematic discharge of rivers and industrial waste is scaled with regard to volume $\left[\left(L_{r}\right)^{2} y_{r}\right]$ per unit time $\left(T_{r}\right)$. Since $T_{r}=I_{r} / \sqrt{T_{r}}$ the total discharge scale $Q_{r}$ is

$$
Q_{r}=\frac{\left(L_{r}\right)^{2} y_{r}}{\left(\frac{L_{r}}{\sqrt{y_{r}}}\right)}=I_{r}\left(y_{r}\right)^{3 / 2}=\frac{1}{5.3 \times 10^{7}}
$$

The discharge $Q_{m}=Q_{r} Q_{p}$ is the discharge into the model over the same interval of time as is expressed for the discharge in the prototype. To make this perfectly clear, if the discharge of a river is $\left(10^{n}\right) \mathrm{m}^{3} /$ mean solar day, the scaled discharge into the model must be $Q_{r} \times 10^{n} \mathrm{~m}^{3} /$ mean solar day, not per model "day." 

The characteristic kinematic scaling factors used in the Barataria Bay model construction and operation are as follows:

$L_{r}$

$\mathrm{y}_{\mathrm{r}}$

$D_{v}=L_{r} / y_{r}$

$T_{r}=I_{r} / \sqrt{y_{r}} \quad$ time scale

$V_{r}=\sqrt{y_{r}} \quad$ velocity scale

$Q_{r}=I_{r}\left(y_{r}\right)^{3 / 2}$

Pr

$u_{r}=0.025 v_{r}$

$$
\text { factor }
$$

wind scale horizontal length scale $1 / 20,000$

vertical length scale $1 / 192$

vertical distortion

discharge scale (total) $1 / 53,000,000$

kinematic density scale $1 / 1$
$192 / 20,000=1 / 104$

$1 / 1440=1 \min . / "$ day"

$1 / 13.8(3)=3.65 \mathrm{~cm} / \mathrm{sec} /$ "kt"

(empirical calibration)

The approximate physical dimensions of the Barataria Bay model are given to provide some acquaintance with the scale of the model experiments. About six months of effort by sixteen part- and fulltime craftsmen were required to build the model and its auxiliary equipment.

over-all north-south length of modeled surface over-all east-west length of modeled surface maximum depth in Gulf portion

Characteristic depth in Barataria Bay/-Grande Ecaille portion height of land above msl. material routed away to form modeled surface volume of water in system weight of flooded model dimensions of model working space volume of model and auxiliary equipment electric motors (synchronous) electric motors (controllable speed) power consumption (lighting) power consumption (total mechanical)
8.0 feet

8.0 feet

0.5 feet

0.04 foot 0.02 foot 600 lbs. 20 cubic feet 2000 lbs. $20 \times 20 \times 20$ feet 3000 cubic feet

4

12

6000 watts

7500 watts 



\section{RECONCILIATION OF KINEMATIC AND DYNAMIC PROCESSES}

The considerations preceding the table of scales have not included the scaling of density and wind velocity. The scaling of density influences mixing, which is essentially a dynamic process, and the scaling of wind velocity involves several considerations which are most accurately reconciled by an empirical process in the present state of the model making art.

Density scales:- The circulation in estuaries frequently involves the mixing of two or more water masses of different density. In direct kinematic (Froude) scaling these density differences should be maintained $1 / 1$ in a model. The $1 / 1$ scale of densities leads to satisfactory results if the problem involves only the kinematics of incursion. If, for instance, a large mass of salt water flows in from the sea under fresh water as in a lagoon or estuary, the velocities, volumes and times of arrival of the main water masses are correct, but the mixing of the two water masses is usually quite dissimilar and unrealistic. The water masses tend to retain their identity too long in the model and to stratify. This result is inevitable, for a fractional scale kinematically similar model can rarely be made to possess a $1 / 1$ ratio of both

5/ While it is customary to consider density in model theory, it is usually simpler to measure specific gravity with respect to pure water at some standard temperature in experimental work. The specific gravity so measured is numerically equivalent to density. 

Froude and Reynolds numbers with its prototype. In the scales of oceanographic models the Reynolds numbers of flow in the model are often several orders of magnitude lower than corresponding values in the prototype.

In the Barataria Bay model two instances of deficient mixing are potentially present due to stratification accompanying (I) the density difference between the predominantly fresh drainage of rainfall into the northwestern part of Barataria Bay via Bayou St. Denis and Grand Bayou, and (2) the density difference between the ambient water of the Barataria Bay-Lake Grande Ecaille system and bleed water. Since there is at present no useful simultaneous solution to the problem of combined Froude and Reynolds density scales a choice was made in the first case in favor of the reciprocal Reynolds scale for increased realism of the circulation, and in the second to abide with the unmodified Froude scale since the injected volumes of bleed water exert a truly insignificant effect on the circulation.

The density difference between fresh water drainage and the ambient density of Barataria Bay water is amply sufficient to cause the fresh water to float on the surface of the ambient water as a thin but very extensive sheet. This tendency exists in nature to the same extent but it is overcome by the mixing processes associated with higher Reynolds numbers of flow, higher eddy diffusivity, and mixing by wave action.. In nature the several mixing processes carry the buoyant fresh water downward 

to form a homogeneous vertical mixture and are so vigorous that the also carry bottom silt upward in sufficient quantities to maintain a high degree of turbidity. In order to reproduce these effects in a model it is necessary to reduce the work to be done by the existing turbulence and associated eddy diffusion. The work to be done is lessened by reducing the density difference empirically by an amount roughly equivalent to the vertical distortion. The necessary change of density difference was actually found to be about two orders of magnitude. This factor reduces the initially small density difference $(0.012)$ to such an uncontrollably small magnitude $(0.00012)$ that the desired condition is more reliably represented by a homogeneous system. Thus the small volume (165 cc/min.) required for the rainfall contribution was pumped from the very large Gulf of Mexico volume and piped into the Little Lake equivalent box attached to the northwest corner of the model. This method of closing the rainfall circulation produced less than 1 part in 2000 error in the tidal circulation and automatically maintained a constant mean sea level.

In the second case, that of the bleed water density scaling, the volumes are insignificant compared with the tidal exchange of the waters they enter, and as bleed water is heavier than the ambient water it tends to sink. While bleed water is about as much heavier than the ambient water per unit volume as the rain water is lighter, and could also be well approximated by a homogeneous fluid, it was considered a more conservative approach if 

the $1 / 1$ dens ity differences indicated by Froude scaling were main6)

tained. This decision causes the simulated bleed water of the model to stratify and collect in depressions and practically to fill these depressions before traveling farther along. It also exaggerates the concentrations of bleed water by retarding its rate of mixing with the ambient water which would otherwise allow it to be carried away with greater rapidity and at greater dilution than the model indicates. When at length, under one-to-one density scaling conditions, the bleed water has reached the steady state of distribution and concentration and is mixing away at a rate equal to its rate of supply, the filaments of a given concentration are somewhat too long, too thin and somewhat too narrow because of the deficient Reynolds number of the flow and dissimilar eddy diffusivity of the gravity controlled kinematic model. It is significant, and a result of this choice, that the principal effect of wind on the bleed water tracer pattern in the model is to drive it upwind on the bottom. Both in nature and in the model thore is a surface layer driven downwind which is usually somewhat thinner and more vigorous than the upwind counter current on the bottom. In nature wave action and more intense eddy diffusion would tend to mix vertically the contents of the two layers, that is, the bleed water accurnulated in the bottom counter current

6/ Question concerning the advisability of departing from Froude scaling arises only in connection with models of very shoal estuaries wherein wave action reaches to the bottom and wind circulation is important. 

would be transported upward into the surface current and the uncontaminated surface water transported downward to dilute the concentrations present in the bottom counter current, and thus drive a more dilute solution of bleed water in both directions at once.

Wind velocity scales:- Wind stress on a model surface cannot be scaled from purely kinematic considerations because the factors concerned are primarily dynamic, and far from perfectly understood in nature. The transfer of energy by wind stress on the water surface involves considerations of at least the roughness of the interface, the temperature difference, temperature structures, eddy diffusivities, and densities of the two media, and probably the condition of the water surface skin. The surface roughness produced by simulated winds over small oceanographic models is usually too small due to the development of predominantly capillary waves rather than gravity waves. Capillary waves do not white-cap readily and they move faster as their lengths diminish while gravity waves move slower as their lengths diminish. Furthermore, to be in scale a modeled wave would have to have a scaled height $y_{r}$ and a scaled length $I_{r}$ in a distorted model. This latter requirement is often a physical impossibility. In view of all these difficulties it has been the practice to scale the model "wind" by scaling the translation effects it produces on the water surface. Oceanographic data indicate that where the fetch is greater than an equivalent of 5 to 10 thousand meters 

and a steady state exists, an appreciable thickness of the surface water will be moving at a speed in the order of 2 to 3 per cent of the steady wind speed. In the Barataria Bay model the average steady wind speeds in each of the cardinal and intercardinal directions were computed from data supplied by the Grand Isle station of the U. S. Weather Bureau. The blowers were driven at the proper rate to produce a $V_{r} s c a l e d$ surface water motion equivalent to 2.5 per cent of the average wind speed wherever the fetch was great enough in each case. Every precaution was taken during the wind calibration and the model tests to have the model surface scrupulously clean so that the surface tension of the water skin maintained a uniform value and the rigidity of the skin was as low as possible. Before each test the surface was cleaned first by an ionic detergent and thereafter by a scummer. Tests were not begun until the detergent had had time to hydrolize and diffuse into the volumes of water below the surface. Since the action of the surface active agent is essentially monomolecular in thickness the resulting volume contamination was truly negligible. The average wind velocities were calculated from statistics for the year 1946 prepared by the New Orleans Office, U. S. Weather Bureau, from observations made at Grand Isle, Louisiana, and are as follows: 



$\begin{array}{llllll} & \begin{array}{l}\text { Wind } \\ \text { direc- } \\ \text { tion } \\ \text { symbol }\end{array} & \begin{array}{l}\text { Wind } \\ \text { direc- } \\ \text { tion }\end{array} & \begin{array}{l}\text { Average } \\ \text { wind } \\ \text { speed } \\ \text { (knots) }\end{array} & \begin{array}{l}\text { Water } \\ \text { speed } \\ \text { (knots) }\end{array} & \begin{array}{l}\text { Variac } \\ \text { (volts) }\end{array} \\ \text { Average spring-summer } & \text { WR } & 140^{\circ} \mathrm{T} & 4.0 & 0.10 & 32.5 \\ \text { Average southeast wind } & \mathrm{SE} & 135^{\circ} \mathrm{T} & 9.0 & 0.22 & 41.0 \\ \text { Average autumn-winter } & \mathrm{SR} & 085^{\circ} \mathrm{T} & 7.8 & 0.20 & 39.0 \\ \text { Average east wind } & \mathrm{E} & 090^{\circ} \mathrm{T} & 10.0 & 0.25 & 40.0 \\ \text { Average northeast wind } & \mathrm{NE} & 045^{\circ} \mathrm{T} & 10.0 & 0.25 & 47.0 \\ \text { Average north wind } & \mathrm{N} & 000^{\circ} \mathrm{T} & 11.3 & 0.28 & 42.0 \\ \text { Average northwest wind } & \mathrm{NW} & 315^{\circ} \mathrm{T} & 9.5 & 0.24 & 41.0 \\ \text { Average west wind } & \mathrm{W} & 270^{\circ} \mathrm{T} & 8.9 & 0.22 & 39.0 \\ \text { Average southwest wind } & \mathrm{SW} & 225^{\circ} \mathrm{T} & 7.6 & 0.20 & 37.0 \\ \text { Average south wind } & \mathrm{S} & 180^{\circ} \mathrm{T} & 8.6 & 0.21 & 38.0\end{array}$

The direction of the average spring-summer wind being within $5^{\circ}$ of southeast, the two directions were not distinguished and the higher average wind velocity was used as one test. The same distinction was omitted in the case of the average autumn-winter and the east wind which also differ in direction by $5^{\circ}$. Thus the actual wind velocities and directions used in the model tests were as follows:

\begin{tabular}{|c|c|c|c|c|c|}
\hline & $\begin{array}{l}\text { Wind } \\
\text { direc- } \\
\text { tion } \\
\text { symbol }\end{array}$ & $\begin{array}{l}\text { Wind } \\
\text { direc- } \\
\text { tion }\end{array}$ & $\begin{array}{l}\text { Average } \\
\text { wind } \\
\text { speed } \\
\text { (knots) }\end{array}$ & $\begin{array}{l}\text { Water } \\
\text { speed } \\
\text { (knots) }\end{array}$ & $\begin{array}{l}\text { Variac } \\
\text { (volts) }\end{array}$ \\
\hline "no wind" run to equilibrium & 0 & -- & 0.0 & 0.00 & 00.0 \\
\hline Spring-summer equilibrium & SR & $1400 \mathrm{~T}$ & 9.0 & 0.22 & 41.0 \\
\hline Autumn-winter equilibrium & WR & $085^{\circ} \mathrm{T}$ & 10.0 & 0.25 & 40.0 \\
\hline Northeast wind equilibrium & NE & $045^{\circ} \mathrm{T}$ & 10.0 & 0.25 & 47.0 \\
\hline North wind equilibrium & $\mathrm{N}$ & $000^{\circ} \mathrm{T}$ & 11.3 & 0.28 & 42.0 \\
\hline Northwest wind equilibriun & NW & $3150 \mathrm{~T}$ & 9.5 & 0.24 & 41.0 \\
\hline West wind equilibrium & W & $260^{\circ} \mathrm{T}$ & 8.9 & 0.22 & 39.0 \\
\hline Southwest wind equilibrium & SW & $225^{\circ} \mathrm{T}$ & 7.6 & 0.20 & 37.0 \\
\hline South wind equilibrium & $S$ & $180^{\circ} \mathrm{T}$ & 8.6 & 0.21 & 38.0 \\
\hline
\end{tabular}



The first three of these runs probably have greatest significance because they show first the action of the tides and rainfall in distributing the bleed water and then how the average conditions of the two major seasonal wind directions and forces influence this distribution. In nature the wind over Barataria Bay apparently does not often blow long enough from any of the nonprevailing directions to re-establish a corresponding equilibrium distribution of bleed water. Each of the wind runs in the model was carried through a little more than one semi-monthly change of the diurnal tide sequence. The equilibria represented in the films of the model undergo continuous cyclic modification as the contributions of the tidal, rainfall and wind circulation systems change in relative importance through a two-week period. The drawings of the results of these tests (Appendix III) show the accumulated greatest extension of bleed water pattern and do not represent the distribution at any one time. Distinction has been made between the continuous and intermittent distribution, some of wich is tidel and some due to the changing direction and force of the winds. 



\section{THE TDE MACHINE}

The characteristic circulation in an estuary such as Barataria Bay is directly related to the daily and monthly sequence of tides. The design and calculations of the tide machine were taken from broader considerations than the tides observed in the estuary itself.

For proper representation of tidal currents in open coastline models the tide machine should be located offshore in the modeled portion of the sea. If possible it should be equidistant from all parts of the model coast line so that the cotidal lines of the wave will be initially parallel to the coast and thereafter altered by refraction as in nature. If it is known that the cotidal lines lie initially at some angle to the coast or are normal to it describing a progressive wave moving up or down the coast line, the tide machine and barriers should be located so as to simulate this approach. The more remote the position of the tide machine from the modeled embayment the more changed are the amplitudes of the tidal coefficients of the machine as they are amended to apply at the position offshore. Fortunately this amendment often allows simplification of the tide generator, for some of the important complicating constituents in the tidal records near the openings to estuaries are due to the behavior of a simple wave inside the estuary. Since the modeled estuary will provide these complicating effects of its own accord, they 

should not be introduced twice. A good approximation of the final form of the offshore tide generator components can be obtained from a weighted average of the tidal coefficients at stations offshore or some distance away in both directions along the coast. The complexity and rate of going of a tide generator varies with the character of the tidal trace both within and along the seaward margins of the prototype. The components of a tide machine are determined from the relative importance of each of the several tidal coefficients compared with their sum. Unimportant coefficients of the same tidal species can be collected and introduced as a single component driven at the weighted mean rate. Important species having speed numbers sufficiently close to this weighted mean can be added as well to simplify the mechanism. Separate components must be set apart if their amplitudes and speed numbers are such that they largely determine the characteristic sequence of tides. In middle latitudes near open sea coasts, the tides are predominantly semi-diurnal with more or les $s$ diurnal inequality and a semi-monthly sequence of ranges. If neither the diurnal inequality nor the semi-monthly range sequence is large, the tide can be closely approximated by a single drum of the proper displacenent driven at the weighted mean rate of all the significant semi-diumal species known for that station. If the diurnal inequality is too great to be neglected but the diurnal species are still unimportant, two semi-diurnal drums will suffice. If the diurnal species are important, and have a 

strong semi-monthly sequence of amplitudes, or if the diurnal species are less important but not negligible, three drums will be required to produce an acceptable approximation of the tides. This is the case applicable to Barataria Bay.

The volume of water to be displaced in the model to effect the rise and fall of tides is the volume ratio $\left[\left(L_{r}\right)^{2} y_{r}\right]$ times the volume change (tidal prism) in the flooded areas of the prototype. To calculate the displacements of the tide machine drums it is possible to prorate the scaled volume of the tidal prism among the important harmonic constituents in proportion to the ratio of their amplitudes to the sum of all terns. This process automatically includes and distributes the minor contributions of the neglected components.

The basic data for the Barataria Bay tide machine were taken from

1. Tidal Harmonic Constants (Atlantic Ocean) TH-I Jan. 1942 U. S. Department of Commerce, Coast and Geodetic Survey 2. Tide Tables (Atlantic Ocean) Ser. 671, 1946.

U. S. Department of Commerce, Coast and Geodetic. Survey

The two stations Pensacola, Florida and Galveston, Texas distantly flanking Barataria Bay were chosen as a combination which would be free of conspicuous estuarine resonance and the average signatures of which would be indicative of the characteristic tidal oscillations on the proximal parts of the continental shelf of the Gulf of Mexico. The average of the two sets 

of harmonic coefficients was taken as a first approximation of the open water tidal condition near the coast.

$$
\begin{array}{llllllll}
\mathrm{K}_{1}- & \mathrm{O}_{1} & \mathrm{P}_{1} & Q_{1} & \mathrm{M}_{2} & \mathrm{~S}_{2} & \mathrm{~N}_{2} & \mathrm{~K}_{2}
\end{array}
$$

$\begin{array}{lllllllll}\text { Pensacola } & 0.43 & 0.49 & 0.13 & 0.08 & 0.06 & 0.02 & 0.01 & 0.02\end{array}$

(1934)

Galveston

(1939)

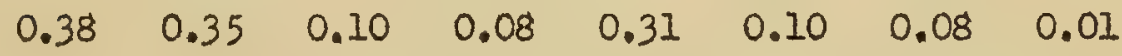

$\begin{array}{llllllll}2 \lcm{0.79} & 0.85 & 0.23 & 0.16 & 0.37 & 0.12 & 0.09 & 0.03 \\ 0.39 & 0.43 & 0.12 & \underline{0.08} & 0.19 & 0.06 & 0.05 & 0.01\end{array}$

Total range 1.24 feet

The small $Q_{1}$ and $K_{2}$ constituents were neglected as individuals and their contributions incorporated in the total displacement of the tide machine drums. Three drums were built and driven through a stroke of 2 inches at the following speeds:

prototype model range radius displ.

$\mathrm{K}_{I} \mathrm{P}_{1}$ drum $24.00 \mathrm{hrs} 60.0 \mathrm{sec} .0 .51$ "It." $5.35 \mathrm{in.} 181.0 \mathrm{in.}^{3}$

$\mathrm{O}_{1}$ drum $25.82 \mathrm{hrs} 64.5 \mathrm{sec} .0 .43$ "ft." $4.92 \mathrm{in.} 152.2 \mathrm{in.} 3$

$\mathrm{M}_{2} \mathrm{~S}_{2} \mathrm{~N}_{2}$ drum $12.21 \mathrm{hrs} 30.5 \mathrm{sec}$. 0.30 "ft." $4.10 \mathrm{in.} 106.0 \mathrm{in.}^{3}$

sum of all contributions 1.24 "feet" $\quad 440.2$ in. $^{3}$

The final tests of this tide machine revealed errors of $3.2 \%$ in

I/ See following page. 

range and $15 \%$ in phase progress due to neglect of the longer period 7)

terms. The $M_{f}$ and $M_{m}$ constituents were partly accounted for in

rate adjustment of the $\mathrm{O}_{1}$ and $\mathrm{K}_{1} \mathrm{P}_{1}$ drums.

$I / K_{I}=$ lunar declinational diurnal constituent, speed $=15^{\circ} .041 / \mathrm{m} . \mathrm{s} . \mathrm{hr}$.

$\mathrm{O}_{1}=1 "$

$P_{I}=$ solar "

"

"1"

, speed $=13^{\circ} .943 / \mathrm{m} . \mathrm{s}$. hr.

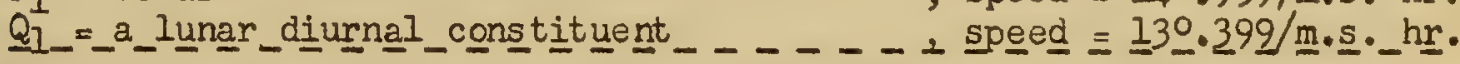

$M_{2}=$ principal lunar semidiurnal constituent, speed $=280.984 / \mathrm{m} . \mathrm{s} . \mathrm{hr}$.

$\mathrm{S}_{2}=$ principal solar semidiurnal constituent, speed $=30^{\circ} .000 / \mathrm{m} . \mathrm{s} . \mathrm{hr}$.

$\mathbb{N}_{2}=$ larger lunar elliptic

" , speed $=28^{\circ} .440 / \mathrm{m} . s . \mathrm{hr}$.

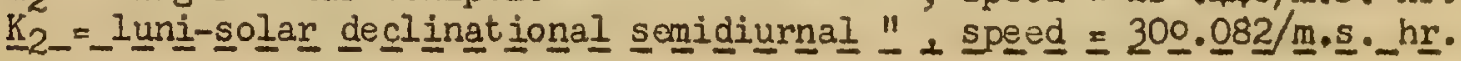

$\mathbb{M}_{f}=$ lunar fortnightly constituent

$M_{m}=$ lunar monthly harmonic constituent

, speed $=10.098 / \mathrm{m} . \mathrm{s} . \mathrm{hr}$.

, speed $=0.544 / \mathrm{m} . \mathrm{s}$. hr. 



\section{KINERAATIC VERIFICATION OF THE MODEL}

The effectiveness and reliability of an offshore tide machine in a model is best evaluated by comparis on with the tide curves at the principal entrances to the estuary and against the accompanying velocities of flow and the times of slack with respect to high and low water in the prototype. These comparisons serve the additional purpose of verifying the kinematic similitude of the whole model.

The verification of the Barataria Bay model was considered consistent with the available data and acceptable when the sequences of times of slack water and maximum currents led or lagged the times of high and low water within one-half "hour" (1.25 sec.) of the times observed in the prototype by H. A. Marmer. Particle traverses were accepted when they agreed within $\frac{1}{4}$ "mile" of Marmer's predictions. Similarly the speeds and directions of the currents were accepted when they fell within less than one quarter "knot" of the speeds and $10^{\circ}$ of the directions reported by Marmer in his reports on THE TIDE IN BARATARI \& BAY and THE CURRENTS IN BARATARIA BAY. Further data on the details of the circulation around the Freeport Sulphur Company property were obtained by the writer or were supplied to him by V. H. Brogdon of the Freeport Sulphur Company. Particular attention was paid to the traverse of particles in Barataria Bay and to the asymmetry of duration of flood and ebb between St. Mary's point and the mouth of Bayou St. 

Denis caused by the rain water drainage through the estuary. The latter is a distinguishing feature of the Barataria Bay circulation because it is indeed a river mouth of rather peculiar shape. Test $A$ is concerned with a detailed study of rainfall drainage and its effect in tending to exclude the waters of both Lake Grande Ecaille and Bay Ronquille from Barataria Bay proper. In the eastern areas there is known to be an unusual division of the directions of ebb currents in the southern part of the Lake Washingt on system which causes the flood and ebb currents to diverge. Ebb currents flow north in part of Lake Washingt on and south in the region nearer Bay Chaland and reverse on the flood. The position of this division in the model was found to be in good agreement with field observations. Through the expedient of interposing the 20 by 20 mesh screens in the seaward passes the calculated rate of going of the tide machine was made acceptable. The calculated fresh water discharge through the little Lake equivalent box led to a correct phase and duration of flood and ebb currents with respect to high and low water at Barataria Pass. Insofar as it is possible to estimate the reliability of a model with respect to field observations, the Barataria Bay model probably possessed a kinematic figure of merit of about 8 on a scale of 10. Since good quality field observations are generally rated at about this same figure of merit, and they alone reveal the behavior of the prototype, the estimated correspondence of the model to the true behavior of the prototype must be between 6 

and 7 on a scale of 10. The higher figure is more likely in this case because the field observations were made by Marmer and his colleagues, whose sagacity is widely acknowledged. 



\section{SPECIFICATIONS OF THE MODEL TESTS}

The construction and verification of the Barataria Bay model discussed up to this point was of a routine nature and followed established precedents in most respects. The principal purpose of the model was to reconstruct the probablo distribution of the maximum quantity of bleed water discharged at any time during the sulphur mining operations of the Freeport Sulphur Company. Toward this end apparatus was constructed which would discharge these maximum specified volumes as traceable material into the model at specified points under continuous discharge conditions. In each case the aim of the experiment was to determine the steady state distribution of the tracer under each of several wind conditions and at each of two dilution figures, namely 1 part in 200 and 1 part in 1000. The specifications for these discharge conditions were supplied by the Freeport Sulphur Company and are as follows:

Test A. Investigate the progress and distribution in the model of fresh water draining through the Barataria Bay estuary from the watershed to the north of Barataria Bay.

Test $B$. Investigate the distribution in the model of a tracer having theoretical kinematic similitude with specified bleed water and capable of showing dilution by the ambient water not in nominal excess of 1 part in 200 and $I$ part in 1000 discharged at the points and at the rates given below: 


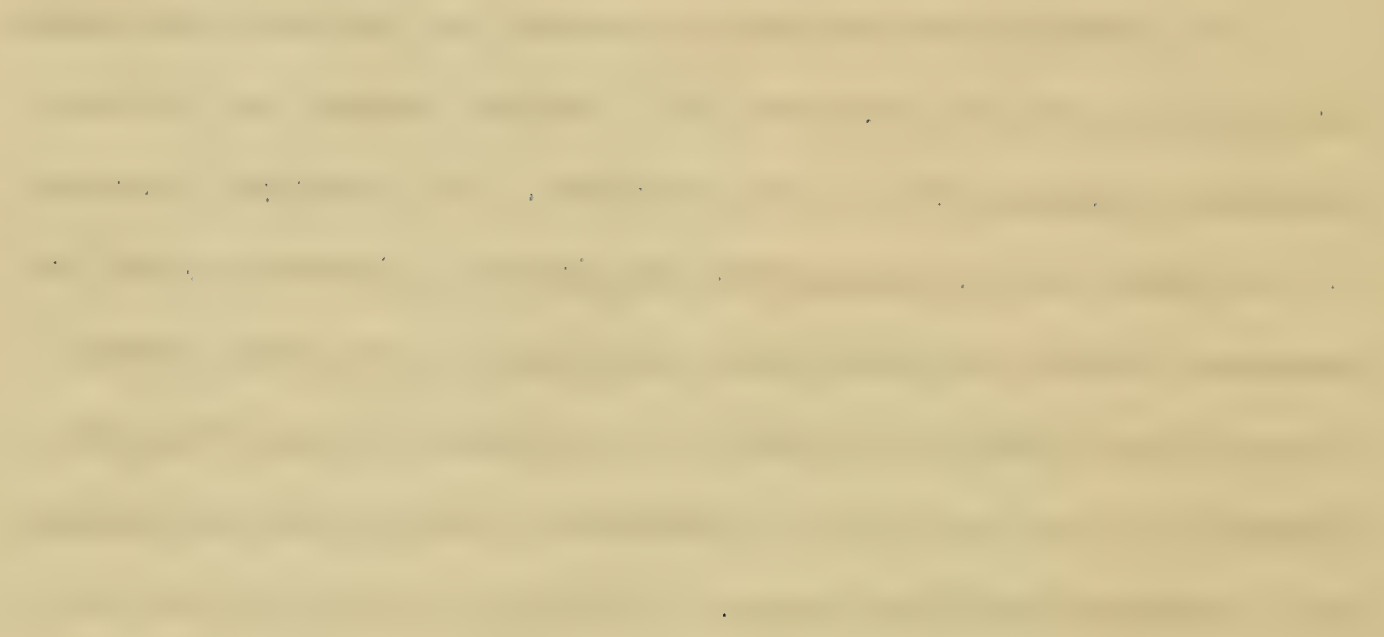


at point VI, 1.4 million gallons per 24 hours, specific $8 /$ gravity $1.057^{\circ}$ at $60^{\circ} \mathrm{F}$,

at point VIII, 1.0 million gallons per 24 hours, specific $8 /$ gravity 1.024 at $60^{\circ} \mathrm{F}$.

Test C. Duplicate Test B with the following additions: Investigate the distribution in the model of a tracer having theoretical kinematic similitude with specified bleed water and capable of showing dilution by the ambient water not in nominal excess of 1 part in 200 and 1 part in 1000 discharged at the point and rate given below: at the head of the Freeport Sulphur Company dilution canal, 1.5 million gallons of bleed water per 24 hours, specific $8 /$ gravity $1.055^{\circ}$ at $60^{\circ} \mathrm{F}$, add at the same point 40 million gallons of Lake Grande Ecaille water per 24 hours, specific gravity $1.012^{8}$ at $60^{\circ} \mathrm{F}$ drawn in the model from the geometrically similar point at which this dilution water is drawn into the pumps in the prototype.

8/ See discussion beginning at the bottom of page 39 . 

VIII. CHEMISTRY OF THE BLEED ViLTER TRACER

In order to meet the specified conditions of the model tests the bleed water was simulated by sulphuric acid of suitable strength and specific gravity and the ambient water by ordinary tap water to which was added a suitable quantity of sodium carbonate and a fraction of a gram of brom cresol green indicator per model volume of about 600 liters. This choice of solutions was made in order to reduce as much as possible any chemical reaction between the solutions and the gypsum (calcium sulphate) plaster of which the model was made and also to avoid alteration of $\mathrm{pH}$ of the embient water through acidification by atmospheric carbon dioxide.

Sulphuric acid introduced to simulate the discharge of bleed water at the specified points and rates in the model necessarily produced a yellow color change in the brom cresol green indicator in solution with it. The color of brom cresol green in alkaline solution is a pleasant blue and the color change as the $\mathrm{pH}$ rises through the range 4.0 to 5.6 is at first green and then bright yellow. The change is abrupt from blue to green and more gradual from green to yellow. As the sulphuric acid flowed with the circulation of the model a certain amount of mixing took place which diluted the sulphuric acid, and at the same time reaction took place with the carbonate ions present in the admixed ambient water. The mixing processes were mainly due to molecular diffusion and microturbulent exchange across the interface between 

the sulphuric acid solution and the ambient water. Thus there was a steep gradient of $\mathrm{pH}$ through this boundary and the color change of the indicator with $\mathrm{pH}$ was at first too abrupt for the individual stages of color to be discerned. As time passed and distance from the source increased the gradient between the acid and ambient alkali usually weakened sufficiently for the individual color changes to be observed. At length when the acid concentration was no longer sufficient to maintain a pH greater than 4.0 the acid became invisible, or neutralized. As pointed out earlier, mixing processes in the model were insufficient to represent the rate of mixing known from field evidence to exist in the prototype, and, since only mixing would permit the process of chemical neutralization to take place, the acid color of the bleed water tracer was generally maintained too long and too far from the source.

Generally speaking the concentration of a sulphuric acid and a sodium carbonate solution can be adjusted so that the intermediate color change from greenish yellow to green will take place when one volume of the acid is thoroughly mixed with any specified number of volumes of carbonate solution. One gram molecular weight of dilute sulphuric acid will be neutralized by one gram molecular weight of sodium carbonate in aqueous solution. Thus if a day's discharge of bleed water is represented by the discharge of a certain volume of liquid containing 0.01 gram molecular weight of sulphuric acid, the presence of this acid will be 

detectable through the yellow or green color of the brom cresol green indicator present until the acid has had an opportunity to mix and react with a volume of ambient water containing 0.01 gram molecular weight of sodiun carbonate. If the volume of 0.01 gram molecular weight of sulphuric acid solution is $1 / 1000$ as great as the volume of ambient water containing 0.01 gram molecular weight of sodium carbonate solution, the boundary across which the acid ceases to be visible represents a dilution of the acid by a factor of 1000 . Thus the color of the acid discharge was characteristic of $\mathrm{pH}$ lower than 4.0 and concentrations greater than 1 part in 1000, and invisible if its dilution exceeded this predetermined value. The same reasoning applies to the 1 part in 200 dilution contour. During operation of the model, samples of the solutions concerned in the bleed water tracer system were titrated repeatedly to end points in both the green and yellow ranges. These values represented the greatest and least measurable dilutions respectively of the acid component and served to define numerically the dilution contours represented by color changes in the model.

The chemical titrations of simulated bleed water against ambient water were referred to a standard sodium carbonate solution of approximately 0.2 normal strength. An intermediate standard sulphuric acid solution of approximately 0,01 normal strength was prepared in order to monitor the concentration of sodium carbonate in the ambient water. The simulated bleed water 

solutions of sulphuric acid were made up in two liter quantities to a strength of approximately 0.5 normal for the 1 part in 1000 tests and approximately 0.1 normal for the 1 part in 200 tests. The exact nomality of each solution was determined through comparison with the standard sodium carbonate solution. The standard sodium carbonate solution in turn was titrated against the intermediate standard acid solution. It was found that the acid prepared for the 1 part in 200 tests was 9.94 times as concentrated as the intermediate standard acid and that the acid prepared for the 1 part in 1000 tests was 50.2 times as concentrated as the intermediate standard acid. The entire mass of ambient water in the model was rendered suitably alkaline by addition of sodium carbonate. It was found that approximately 2.0 milliliters of the intermediate standard acid solution produced the blue-green to blue change of color in the brom cresol green indicator and that approximately 3.1 milliliters of intermediate standard acid produced the green-yellow color change when added to 50 milliliters of the sodium carbonate solution used as the ambient water. This ratio of concentrations was monitored by titrations throughout the model tests. During each test the dilution factor inevitably tended to shift. The aver age dilution factor was calculated for each run and the results of these calculations are tabulated in the log sheets and entered on the smooth plots of the results for each test. Two values are given; the value for the yollcw-green change representing the highest measurable concentration of simulated bleed 

water, and the value for the green-blue change representing the lowest measurable concentration of simulated bleed water. In each case it was the intention to make these values straddle the nominal dilutions specified for the tests. The dilutions were calculated as follows:

$V_{g}=$ volume of Gulf water titrated (50 cc by volumetric pipette) $V_{a}=$ volume of intermediate standard acid used to neutralize $V_{g}$ $V_{b}=$ volume of simulated bleed water equivalent to $V_{g}$

$$
\begin{array}{ll}
\begin{array}{l}
\text { for } \\
\text { l:200 } \\
\text { tests }
\end{array} & \frac{V_{g}}{V_{b}}=9.96 \times \frac{V_{a}}{V_{b}} ; \\
\begin{array}{l}
\text { for } \\
\text { tests } \\
\text { tests }
\end{array} & \frac{V_{g}}{V_{b}}=50.2 \times \frac{V_{a}}{V_{b}}
\end{array}
$$




\section{AUXILIARY PUMPING SYSTEMS}

The scaled quantities of bleed water were pumped through 1/8-inch copper tubes embedded in the plaster monolith. These tubes were terminated at point VI on the north bank of Humble Canal, point VIII on the west bank of the dredge cut, at the head of the dilution canal and on the shore of Lake Grande Ecaille adjacent to the head of the dilution canal (see fig. 14). The first three tubes were attached to a positive displacement pump driven by an electric motor through suitable screw. The fourth tube was used to withdraw the dilution volumes from Lake Grande Ecaille for return at the head of the dilution canal together with the appropriate volumes of simulated bleed water. Thus the hydraulic activities of the Freeport Sulphur Company mining operations having significance in these tests were substantially reproduced in the model.

The kinematic scaling factor of discharge $Q_{r}$ being $1 / 5.3 \times$ 107 per mean solar day, the discharge per man solar minute into the model is $Q_{n}=Q_{p} T_{r}$ or $1 / 7.68 \times 10^{10}$. One million gallons in the prototype is therefore represented in the model by a volume of 0.05 cubic centimeter of liquid or approximately one drop. Pumps that will deliver such small quantities of liquid per minute are difficult to build and to operate. In order to increase the volumes to be discharged by a single pumping unit, and to simplify the maintenance of predetermined differences of 

specific gravity, which in this case wore more significant than specific gravity ratios, permission was obtained to average the specified specific gravities of the simulated bleed waters and to maintain this average difference above that of the ambient water of specific gravity 1.000 at $60^{\circ} \mathrm{F}$ rather than 1.012. Since specific gravity is more easily measured than density and is numerically equivalent to density if comparisons are made at a convenient standard ternperature, usually $60^{\circ} \mathrm{F}$ or $20^{\circ} \mathrm{C}$, the resulting error in the equivalent density ratio was $1.2 \%$, The average difference in specific gravity of the simulated bleed water and ambient water at $60^{\circ} \mathrm{F}$ was taken as $1.045-1.012=1.033$ corresponding closely to that of a $5 \% \mathrm{NaCl}$ solution at the same temperature. Thus the kinematic density difference $0.033 \mathrm{gm} / \mathrm{cc}$, measured in terms of specific gravity under homogeneous temperature conditions equivalent to $60^{\circ} \mathrm{F}$, was adjusted by adding the necessary quantities of $\mathrm{Na}_{2} \mathrm{SO}_{4}$ to the bleed water tracer.

A positive displacement pump had been designed and built to handle the sum of both the simulated bleed water volumes and the dilution volumes, but later it was thought better practice to actually withdraw the dilution volumes from the model since these volumes form a small but possibly significant part of the local circulation. Relieved of this relatively major burden the bleed water pump had to be sun at very slow speeds, and during Test B it was found that accurate proportional delivery of the scaled volunes could not be detected and controlled quickly enough to 
correct the "day-by-day" discharge rates but that the total discharge over a period of a "week" was acceptable. Since the points of discharge in Test B are so close together and united in a common portion of the detailed circulation no significant error in the equilibrium distribution of bleed water could occur as a result of small proportioning errors. In Test C, however, a small proportioning error would be highly significant in the equilibrium distribution of bleed water because the dilution canal is part of a circulation pattern that is distinct from the Humble Canal system. Test $\mathrm{C}$ was, therefore, not sun until a reliable. rotary proportioning device had been built and installed. The total discharge of the bleed water pump was monitored as before by timing the revolutions of the driving motor shaft and screw. The results obtained in all bleed water discharge tests are believed to be representative and consistent in spite of the difficulties enumerated.

Two other pumping systems had significance in the model tests (1) the dilution system which withdrew scaled volumes of water from Lake Grande Ecaille and discharged them as a mixture with properly scaled volumes of bleed water added, and (2) the rainfall pumping system. The bleed water dilution system consisted of a small gear pump driven by a separate electric motor and monitored by counting the number of drops discharged per minute as they fell from a calibrated dropper tip. Since the scaled volumes involved a drop every few seconds it was possible to observe the rate of 

discharge quite readily and maintain acceptable accuracy. Similarly the rain water circulation system involved the discherge of 165 cubic centimeters per minute, a volune equivalent to Narmer's estimate of the average fresh water drainage $\left(450 \times 10^{6} \mathrm{ft} .3 /\right.$ day) into Barataria Bay. This volume was pumped from the Gulf portion and was piped around the outside of the model to the Little Lake equivalent box where it discharged as a small steady stream. This flow was adjusted by means of a needle valve at the pump. The same punp also circulated a continuous stream in the Gulf of Mexico portion of the model through a venturi tube which entrained the Gulf water and produced the characteristic westward trend of the circulation of Gulf past the mouths of the passes into the Barataria Bay estuary. The venturi system was adjusted to produce a steady westward current of 0.25 "knot" along the coast line. 



\section{THE RELIABILITY OF THE MODEL TESTS}

The final figure of merit for all the observations represented in the model tests can be estimated only very roughly. The model itself is rated at 7 on a scale of 10 . The equilibrium distribution of bleed water in the model is estimated at the same figure. Hence the reliability of the bleed water tracer tests must be about 5 on a scale of 10. This figure of merit should be interpreted to mean that the contours of bleed water distribution may be overextended with respect to the actual occurrence in the prototype by a factor of 2 or they may be underextended by a factor of $1 / 2$. The kinematic density scale chosen in connection with simulated bleed water discharge caused the bleed wator to sink unnaturally and inhibit the mixing processes that would otherwise dilute the discharge. With the condition of insufficient mixing applying so heavily to the first possibility and to the estimate of the figure of merit itself, underextension of the dilution contours of bleed water distribution is improbable. 



\section{XI. "FIRST ARRIVLL" TESTS}

In addition to the tests for the equilibrium distribution of bleed water and penetration of fresh water discharge some separate tests were run and photographed to determine the minimum time required for a parcel of water composed of an hour's discharge from point VI in Humble Canal to travel to the head of Bay Ronquille. It was found that the volume of colored ink tracer released at point VI had to be deposited at a very critical moment near the beginning of a tropical ebb tide in order to reach the head of Bay Ronquille before the following flood carried it back toward its starting point. The minimum time required for a parcel to actually reach the head of Bay was approximately seven or eight hours.

Experiments in the field show that the travel time on the tropical flood tide from point VI in Humble Canal to Fisherman's cut on the east side of the Freeport Sulphur Company dredge cut is about five hours. The model confirms this result provided the tracer has neutral density. If the tracer is of kinematically (1/1) scaled density the discharge through Fisherman's cut is delayed until the dredge cut basin is filled and a stratified layer established which is almost level with the threshold of Billet Bay. Following this the travel time is the same for solutions of both neutral and kinematic density. 

Appendix I - Schedule of procedure in model tests.

Preliminary readiness check

1. check titre of ambient water and sea level, clean water surface

2. check specific gravities of ambient water and simulated bleed water for difference of 0.033

3. check rate of fresh water discharge at $165 \mathrm{cc} / \mathrm{min}$.

4. check filling of bleed water pumps with proper acid solution

5. check rate of discharge of bleed water pump

6. blow out bleed water discharge lines and flush with ambient water

7. fill bleed water discharge lines with proper acid solution to point of incipient discharge

8. check rate of going of tide machine

9. check tide recorder for ink and paper supply

10. check wind sock

11. check run code number

12. replace all photofloods and test lighting system, measure illumination

13. check power to wind machines, and alignments

14. check film supplies, cameras and exposures

15. check velocity of flow through Barataria Pass, Bayou St. Denis

Operating duties

Chemist

1. keep running check and log of titre of ambient water

2. operate wind mackines on signal

3. place and remove wind sock on signal

4. change code numbers on signal

5. replace photoflood lamps as necessary

Mechanist

1. keep continuous watch on bleed water discharge rate, dilution pump rate, tide machine rate and fresh water discharge

2. start and stop and label tide recorder on signal

3. start and set elapsed-time clock on signal

4. replace photorlood lamps as necessary 



\section{Photographer}

1. keep continuous watch on framing, exposure, focus and film supply of all cameras, change film as required

2. keep watch on the over-all behavior of the model

3. general instructions for operating $16 \mathrm{~mm}$ time-lapse and 35 $\mathrm{mm}$ Graflex cameras

a. operate $16 \mathrm{~mm}$ time-lapse Bolex with $15 \mathrm{~mm}$ Eastman Cine f-2.7 objective and Type A Kodachrome in 100 foot rolls at the rate of 1 frame per second

b. operate $35 \mathrm{~mm}$ Graflex Photorecord camera with $44 \mathrm{~mm}$ Wollensak f-3.2 objective at air pressure of 22 lbs using 100 foot rolls Type \& Kodachrome at the rate of 1 frame every 7.5 seconds. Synchronize with model clock

c. operate $35 \mathrm{~mm}$ Leica with $127 \mathrm{~mm}$ Wollensak objective f-4.5 at optional intervals during runs to equilibrium and at intervals of approximately one week during wind tests. Include the vicinity immediately adjacent to Freeport Sulphur Company area in field of view. Try to record significant changes or developments of the bleed water patterns

4. make serial photographic record of model tests as follows:

a. run to equilibrium without wind - run both 16 and $35 \mathrm{~mm}$ cameras

b. run to summer wind equilibrium - man both 16 and $35 \mathrm{~mm}$ cameras

c. return to no-wind equilibrium - no photographs

d. run to winter wind equilibrium - run both 16 and $35 \mathrm{~mm}$ cameras

e. return to no-wind equilibrium - no photographs

f. cardinal and intercardinal winds - start 16 and $35 \mathrm{~mm}$ cameras when equilibrium is attained, continue photographing until one-half monthly progression of tides has passed.

5. keep log of photographs and model behavior

Completion duties

1. secure all electrical machinery and lighting equipment

2. flush model free of distributed acid

3. dry off land areas with sponges

4. secure work lights 




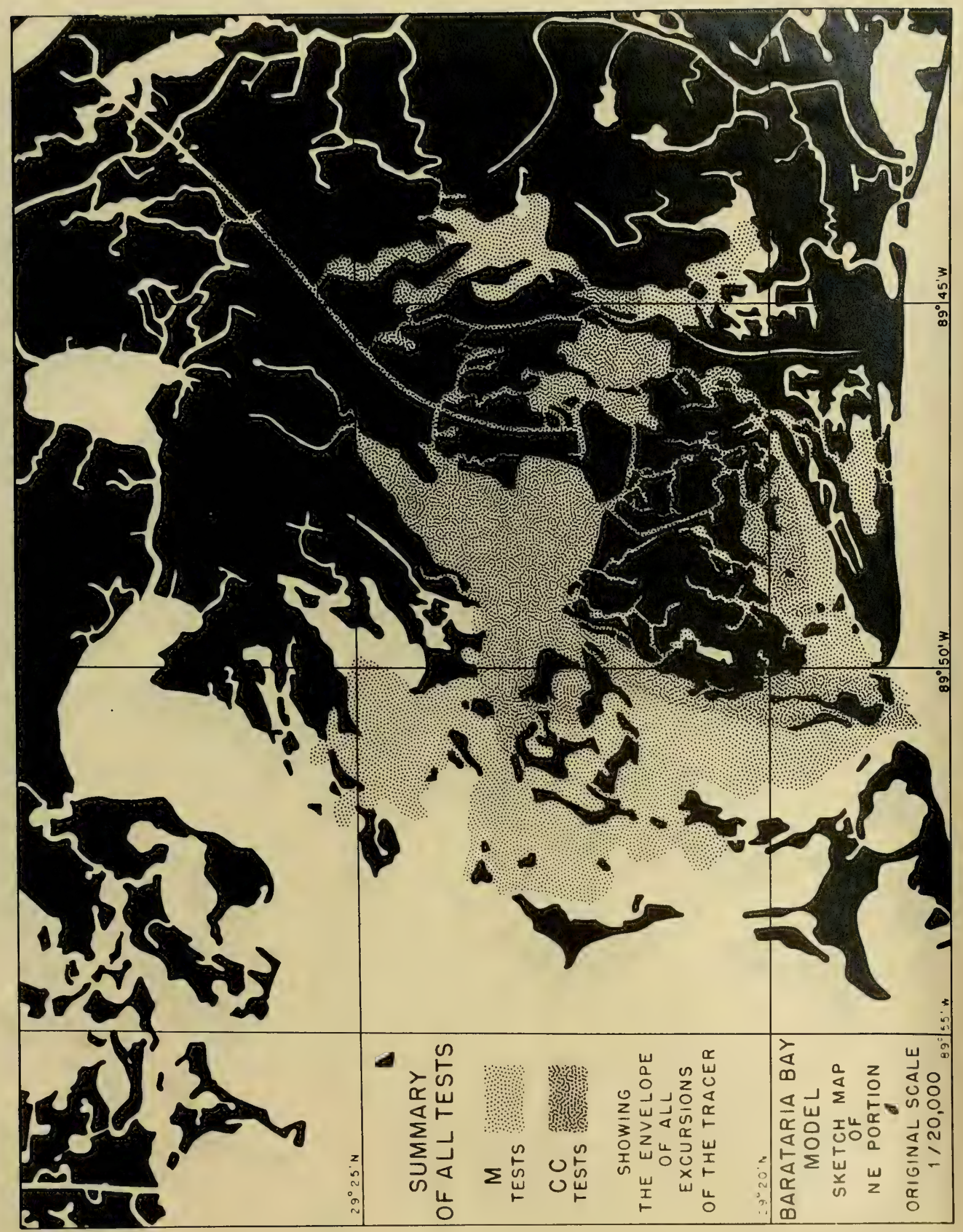





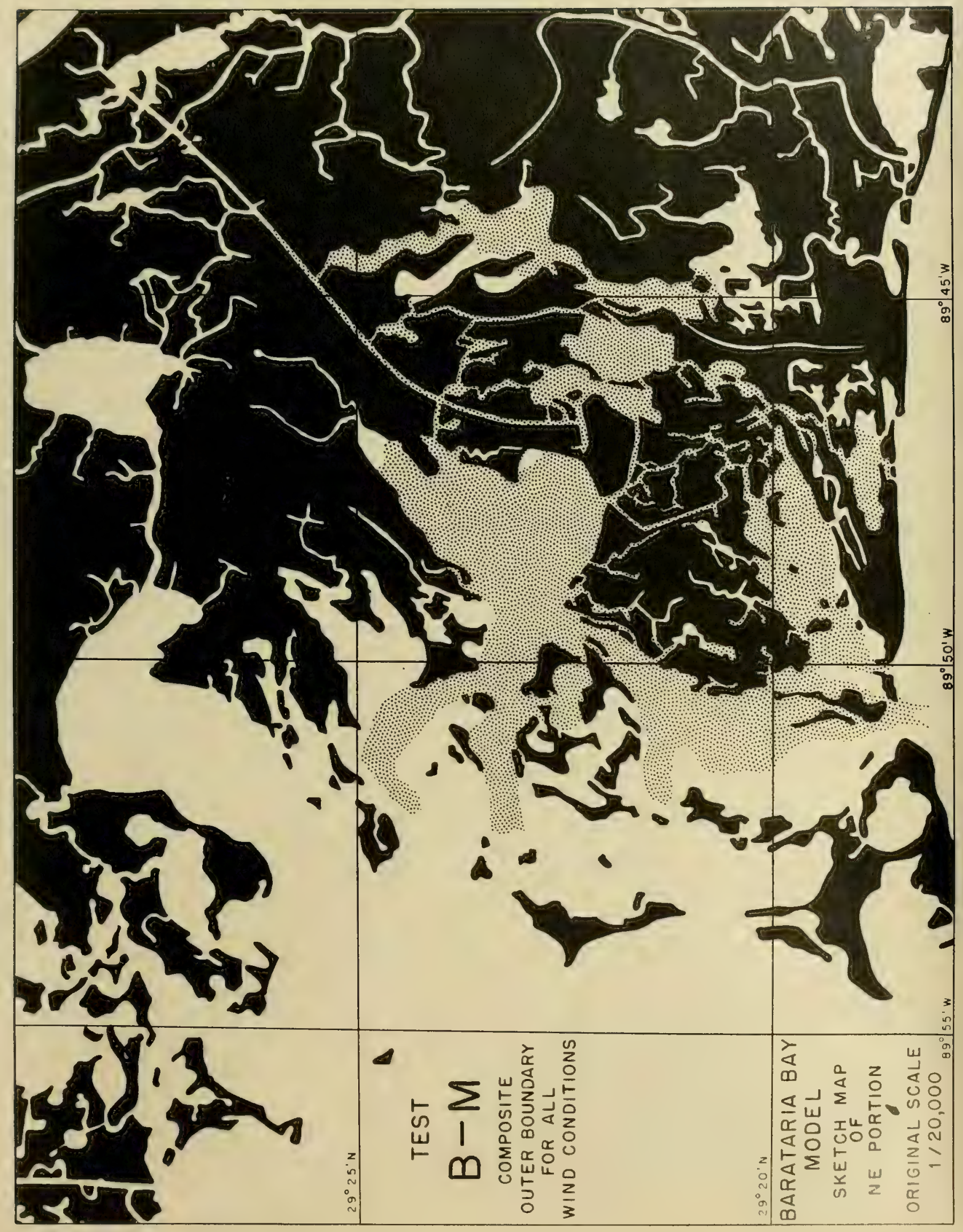





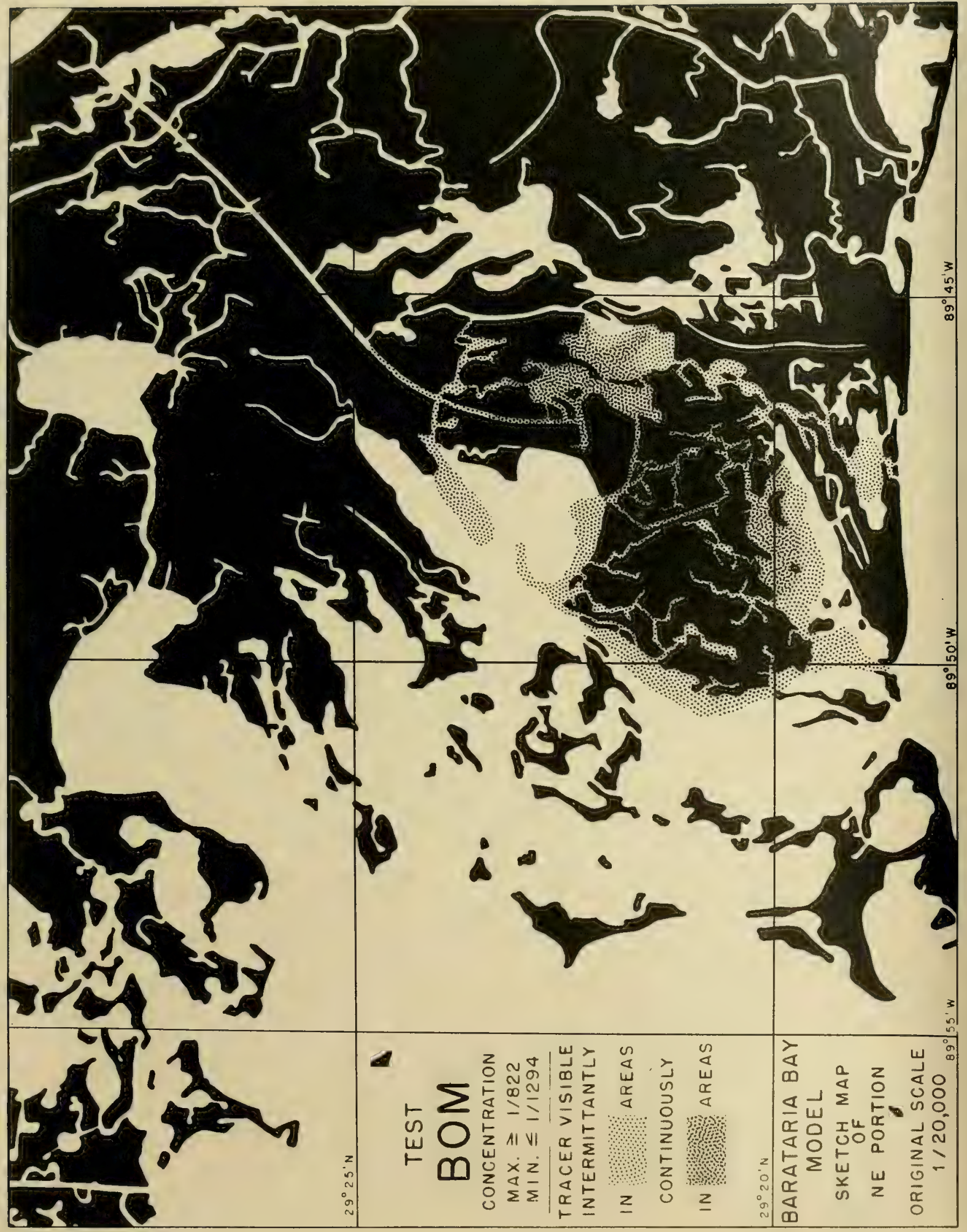





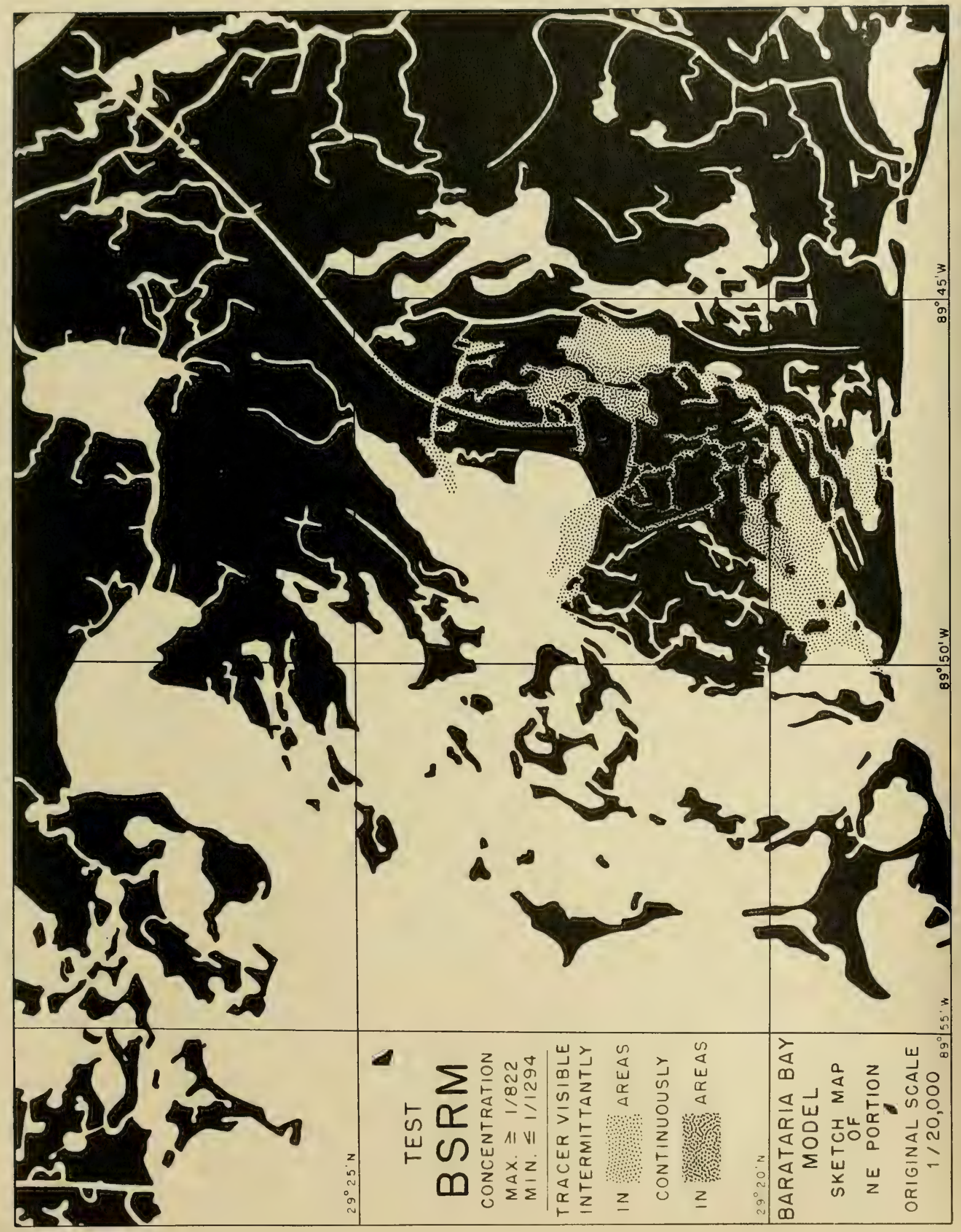





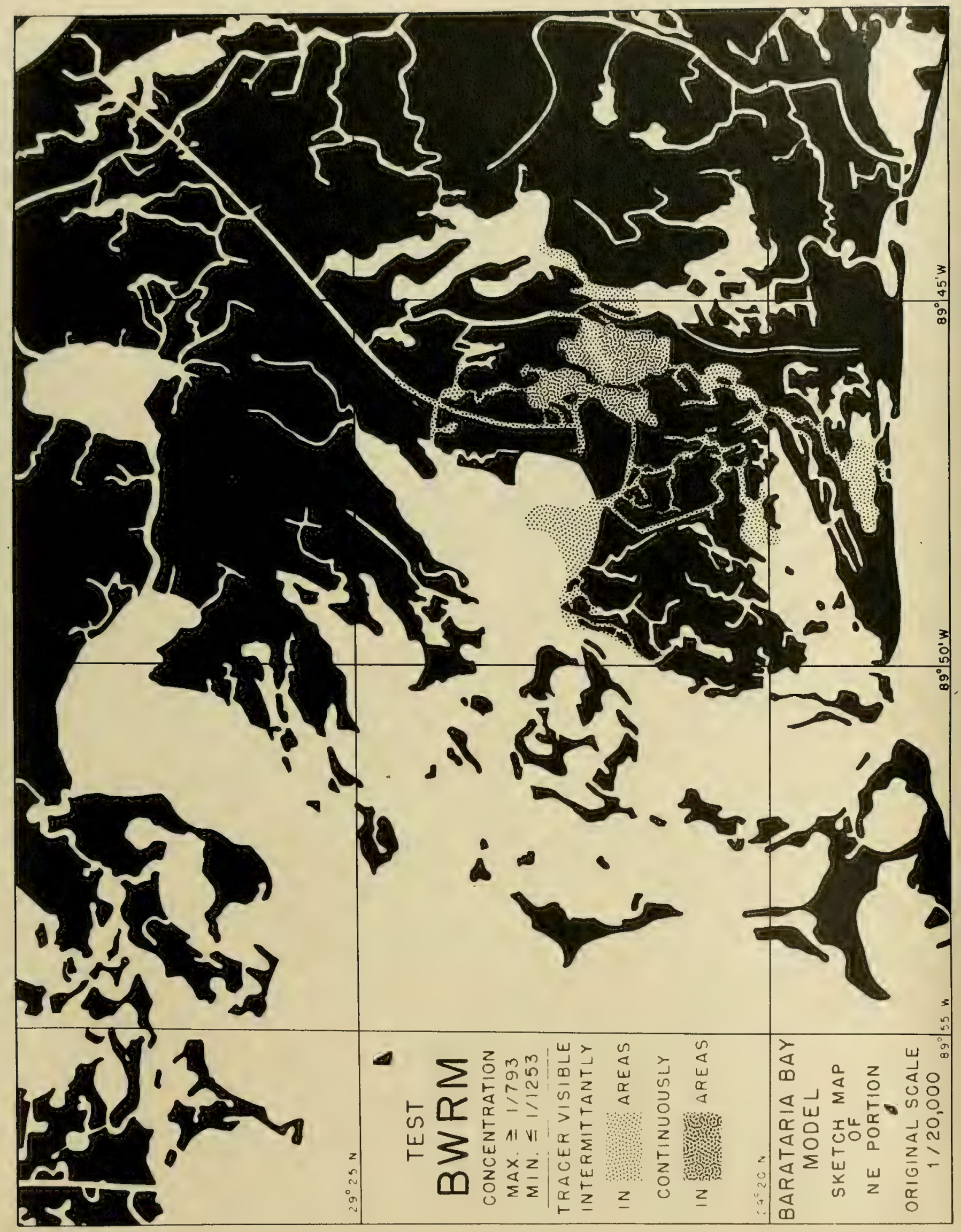





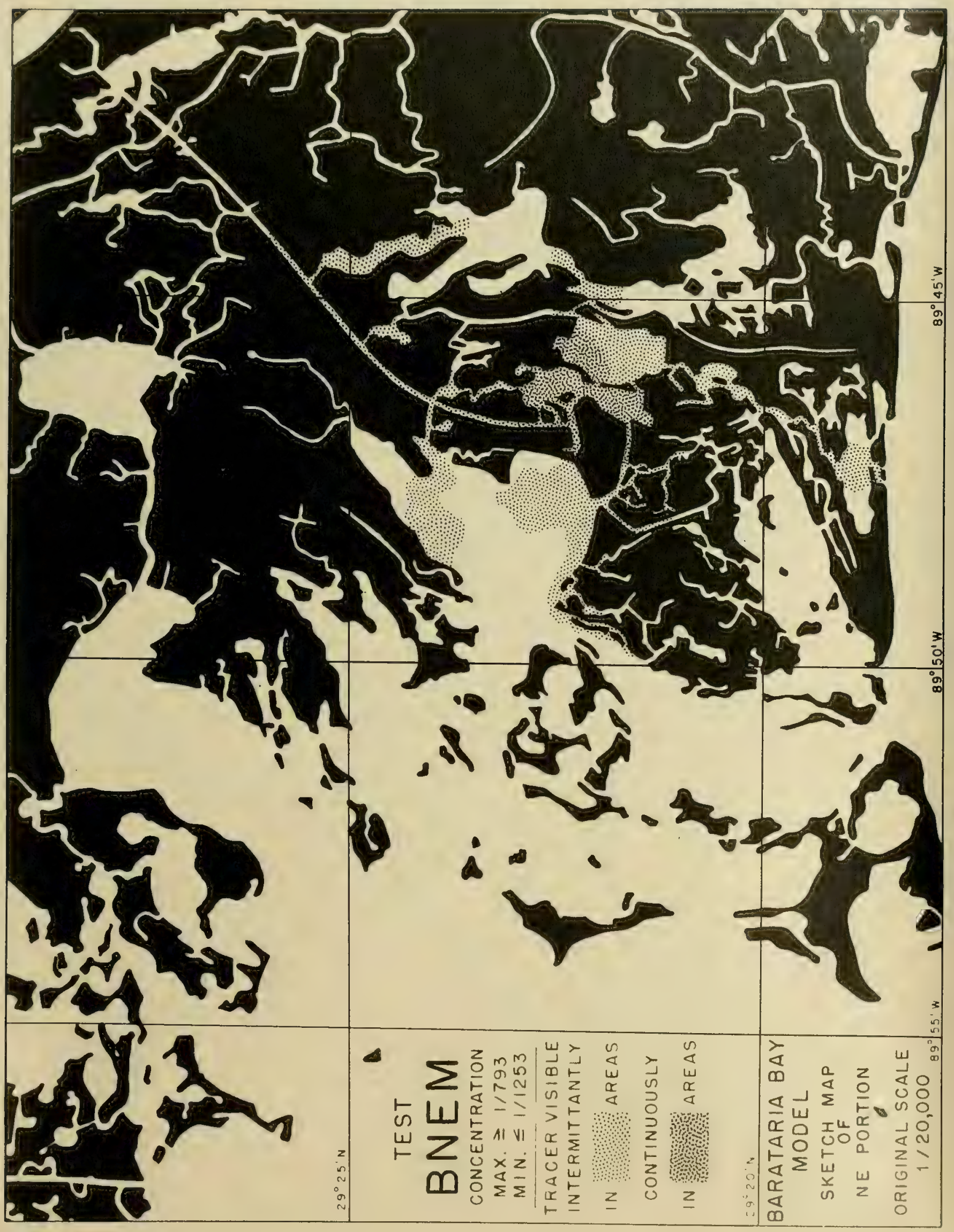





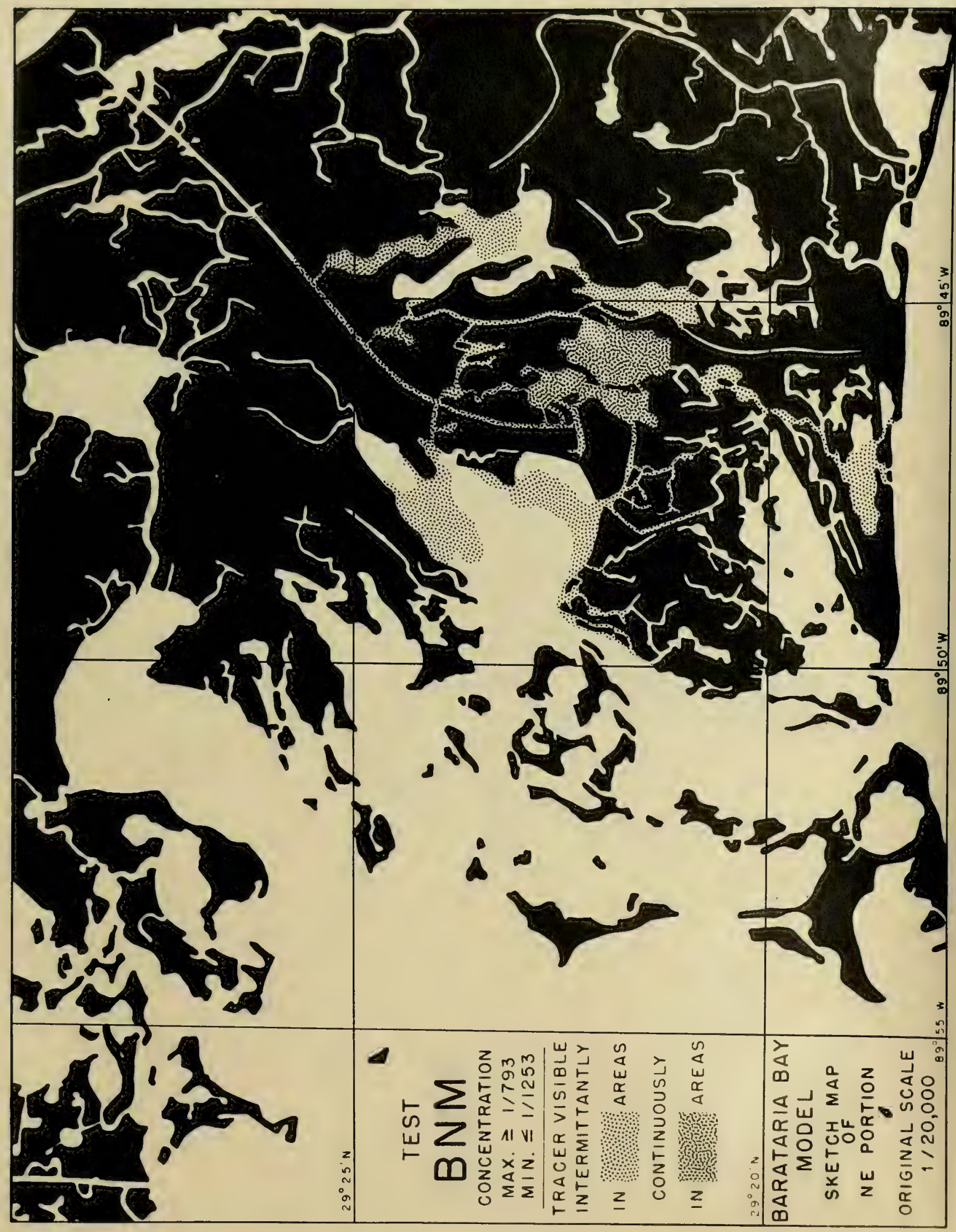





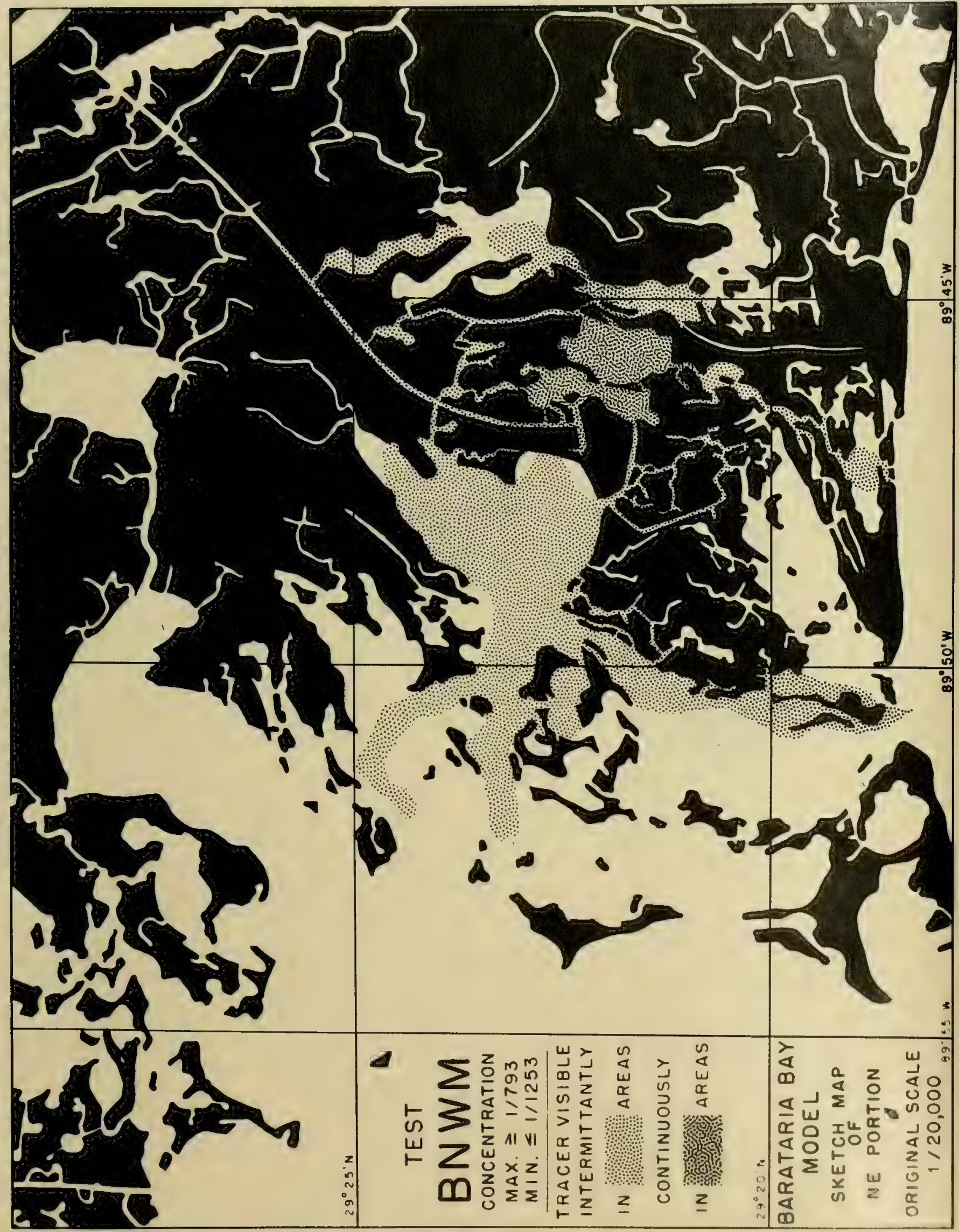





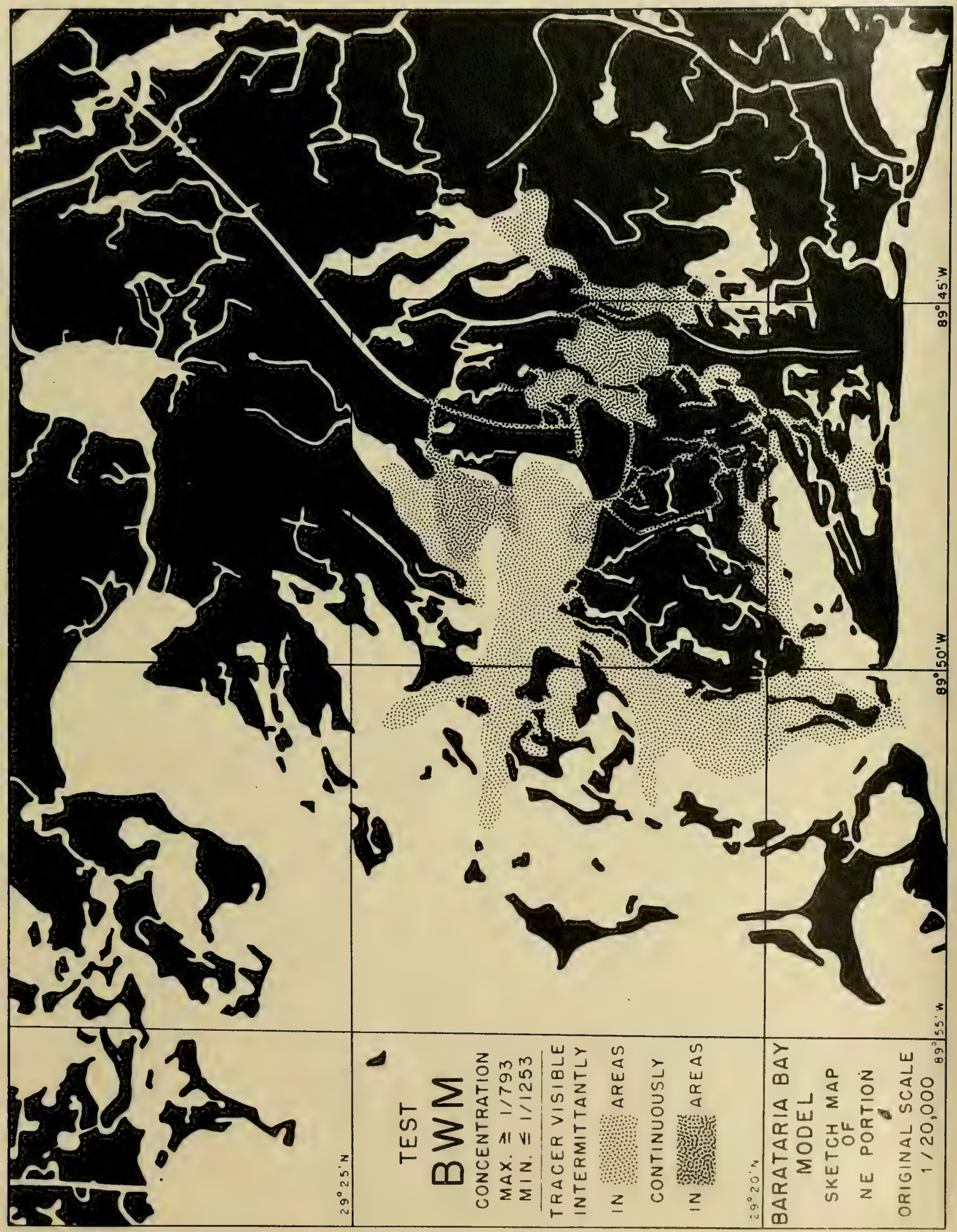





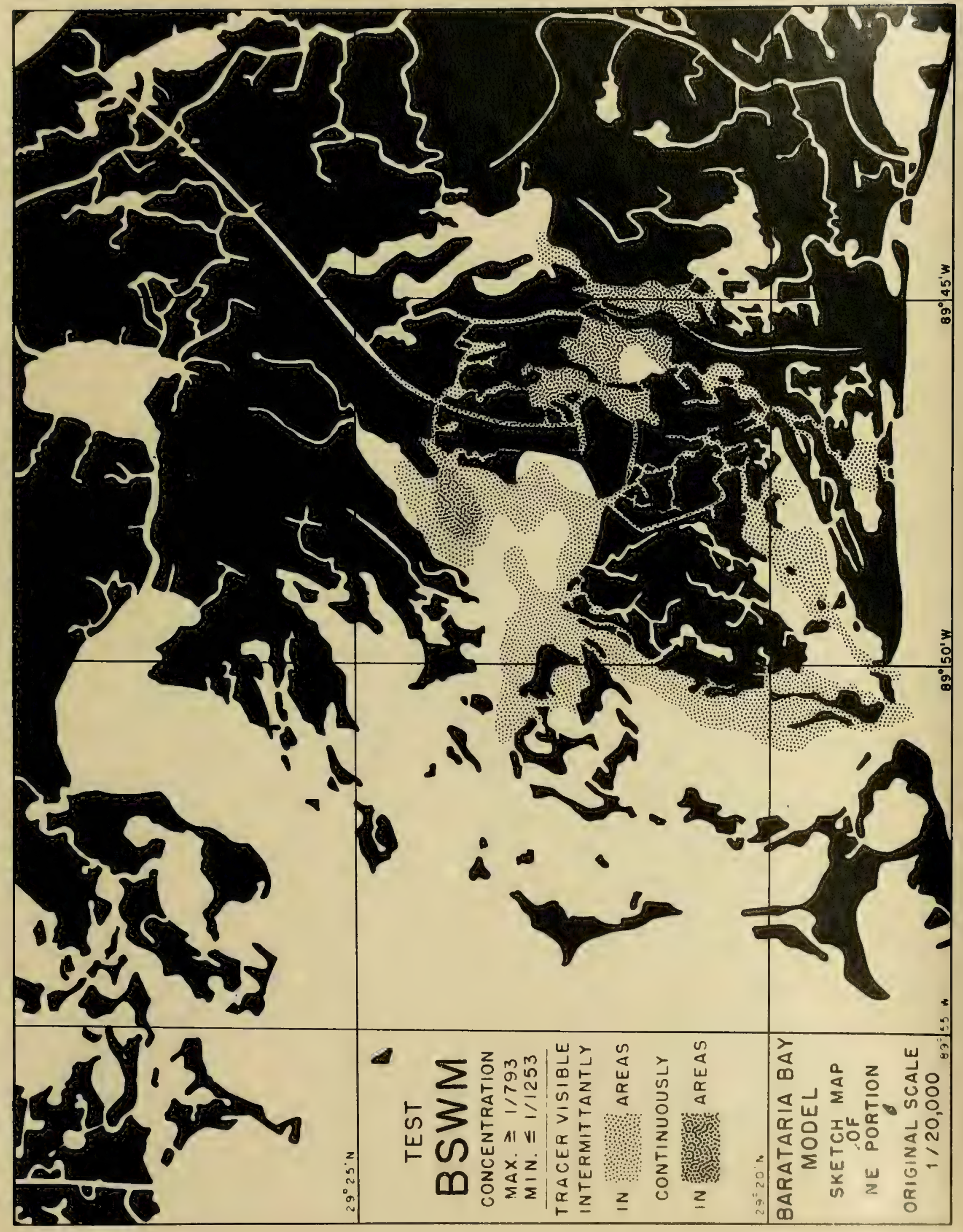





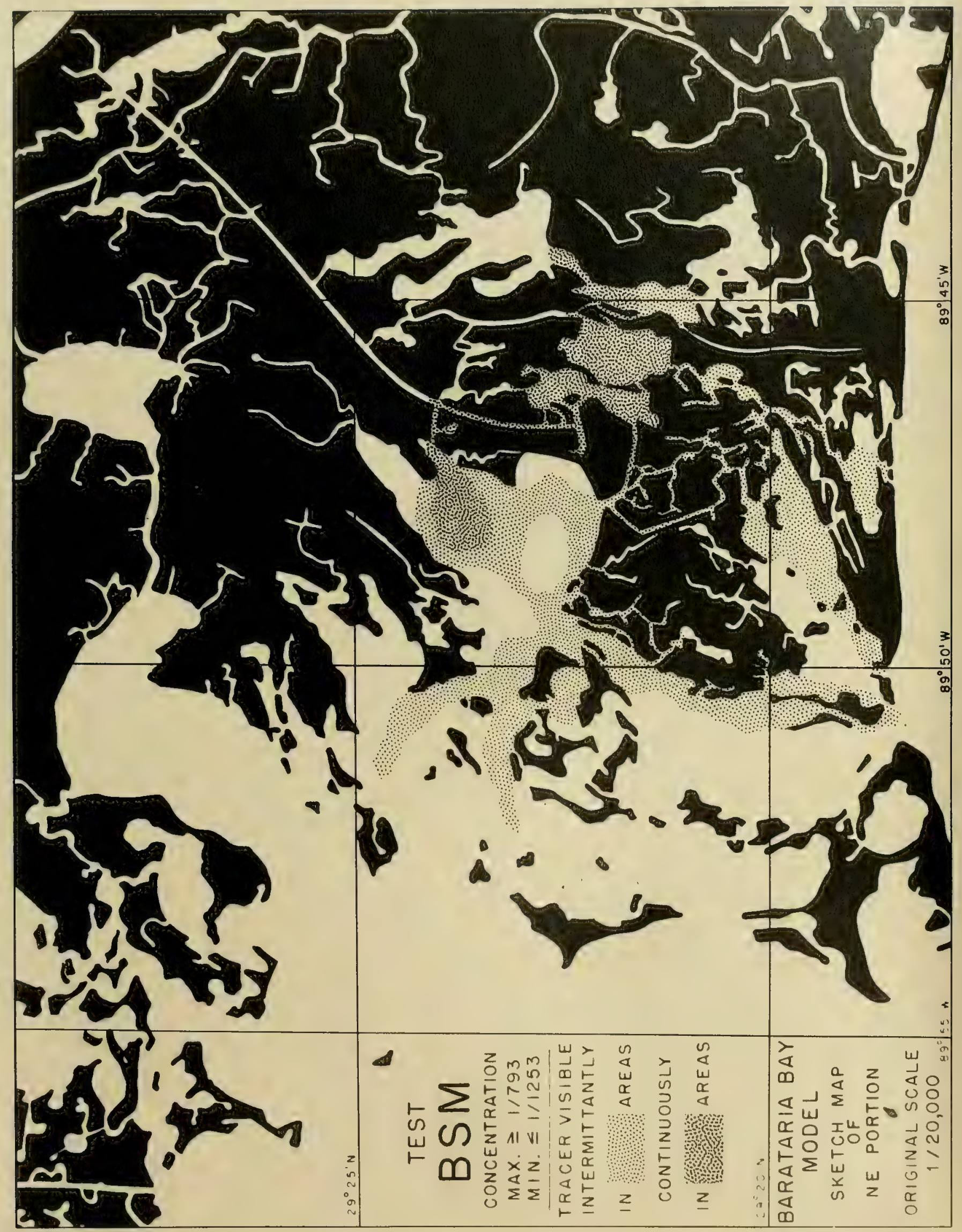





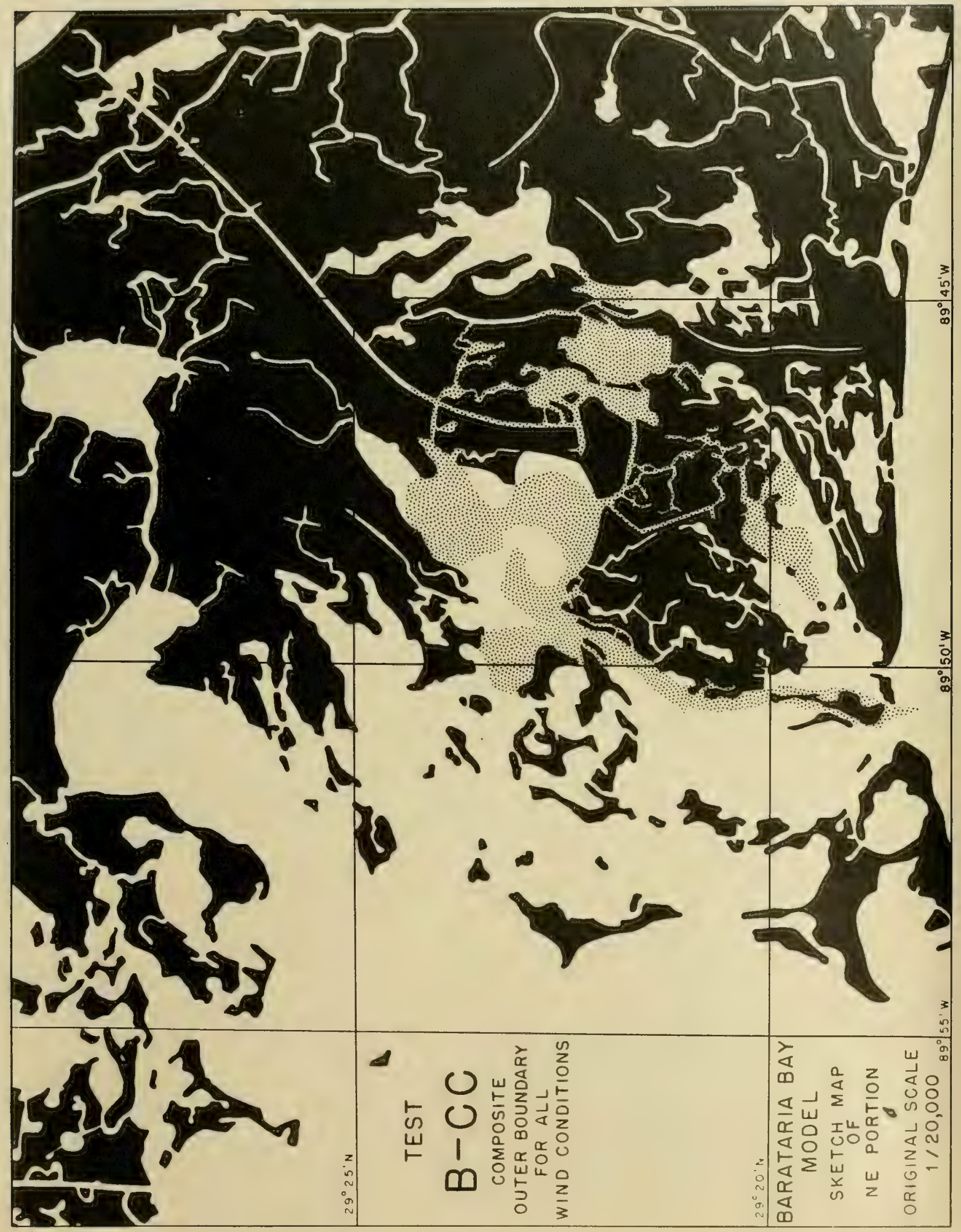





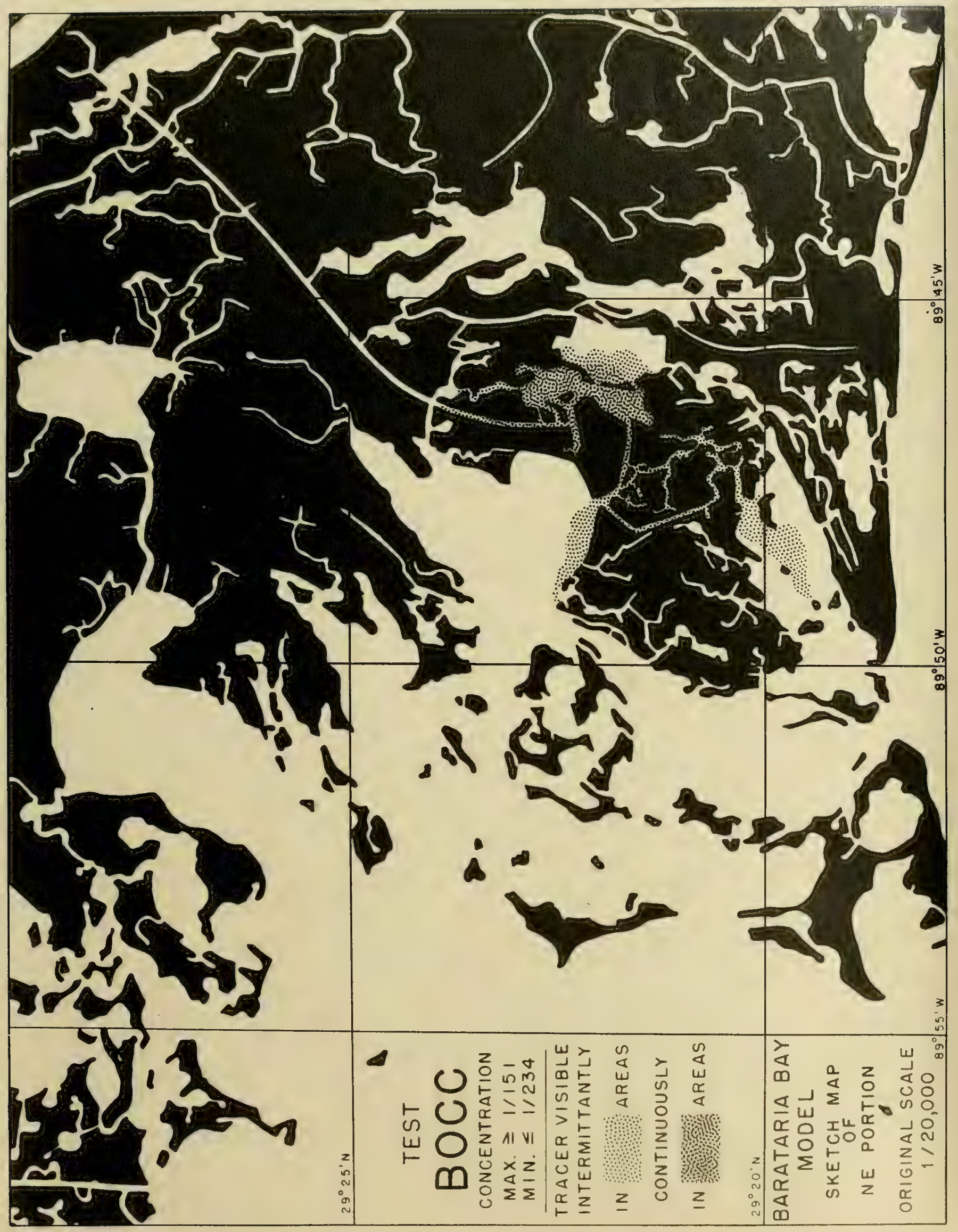





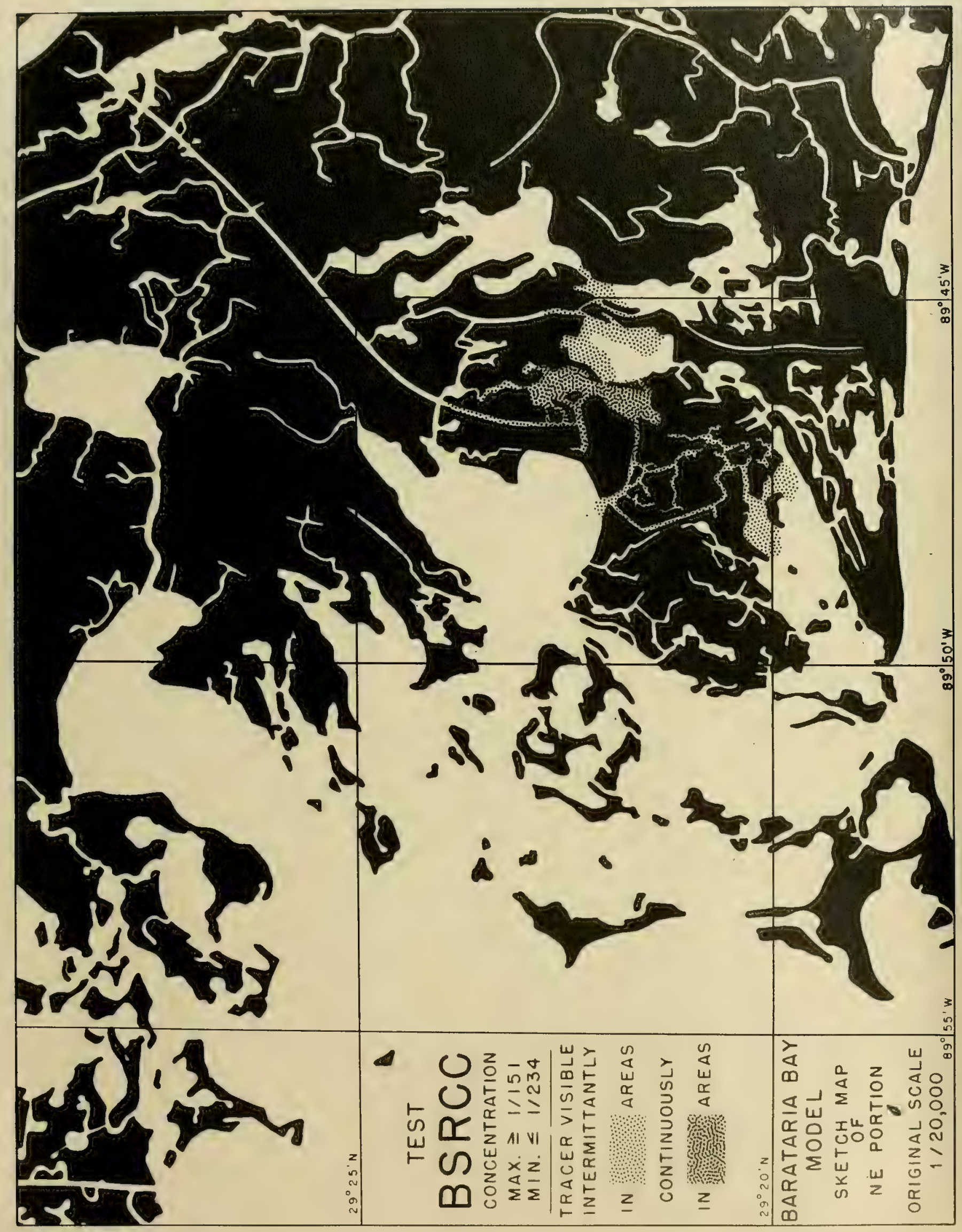





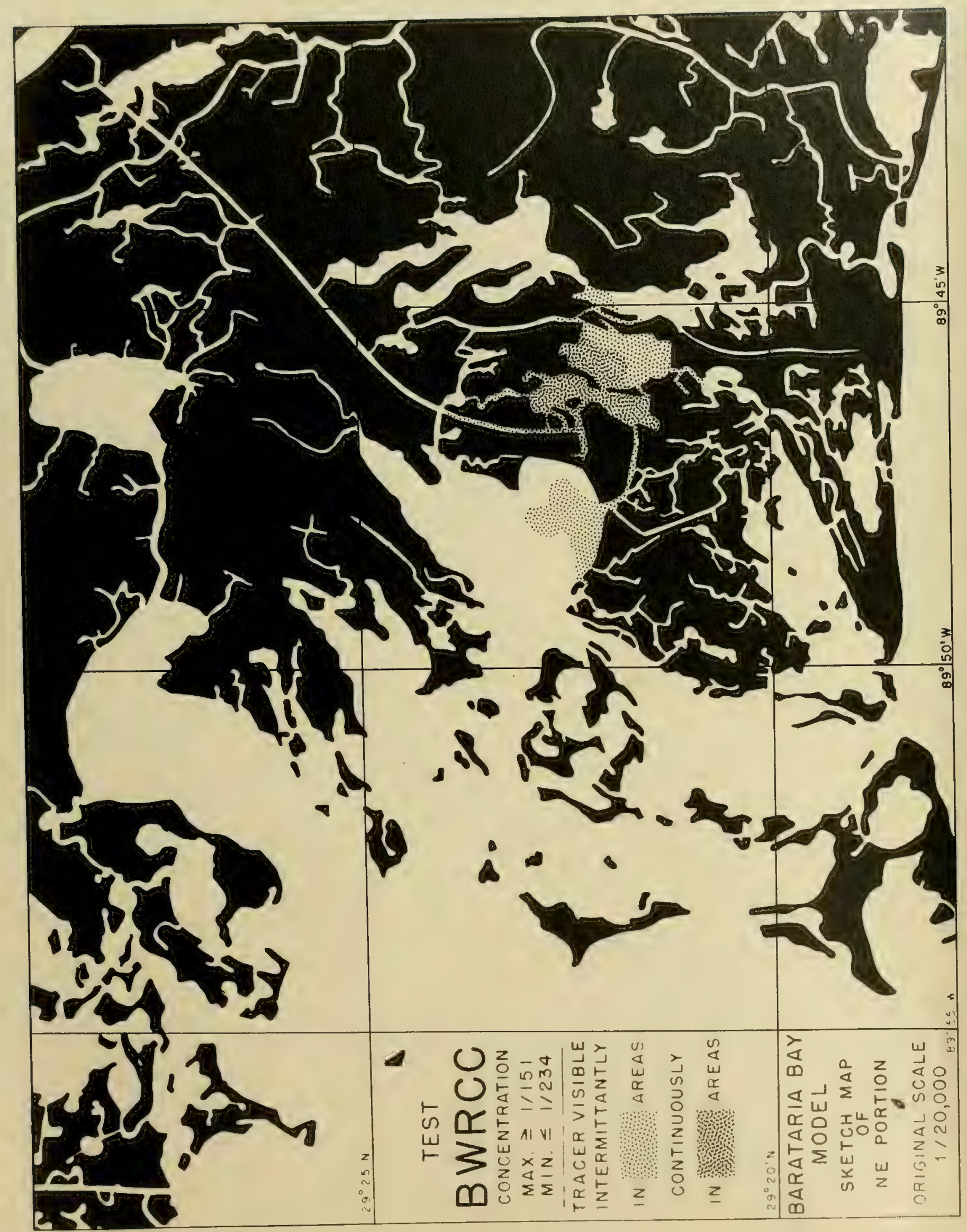





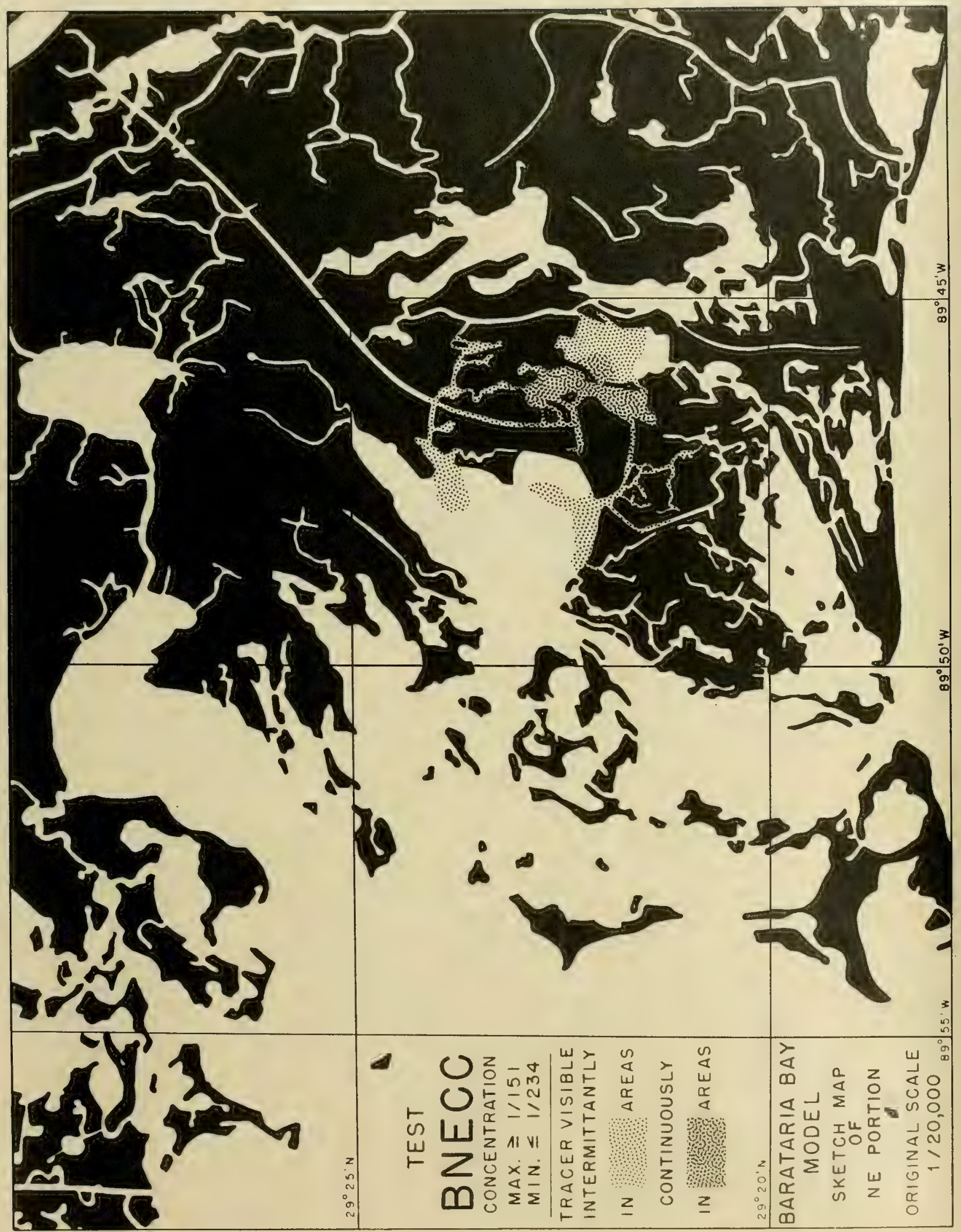





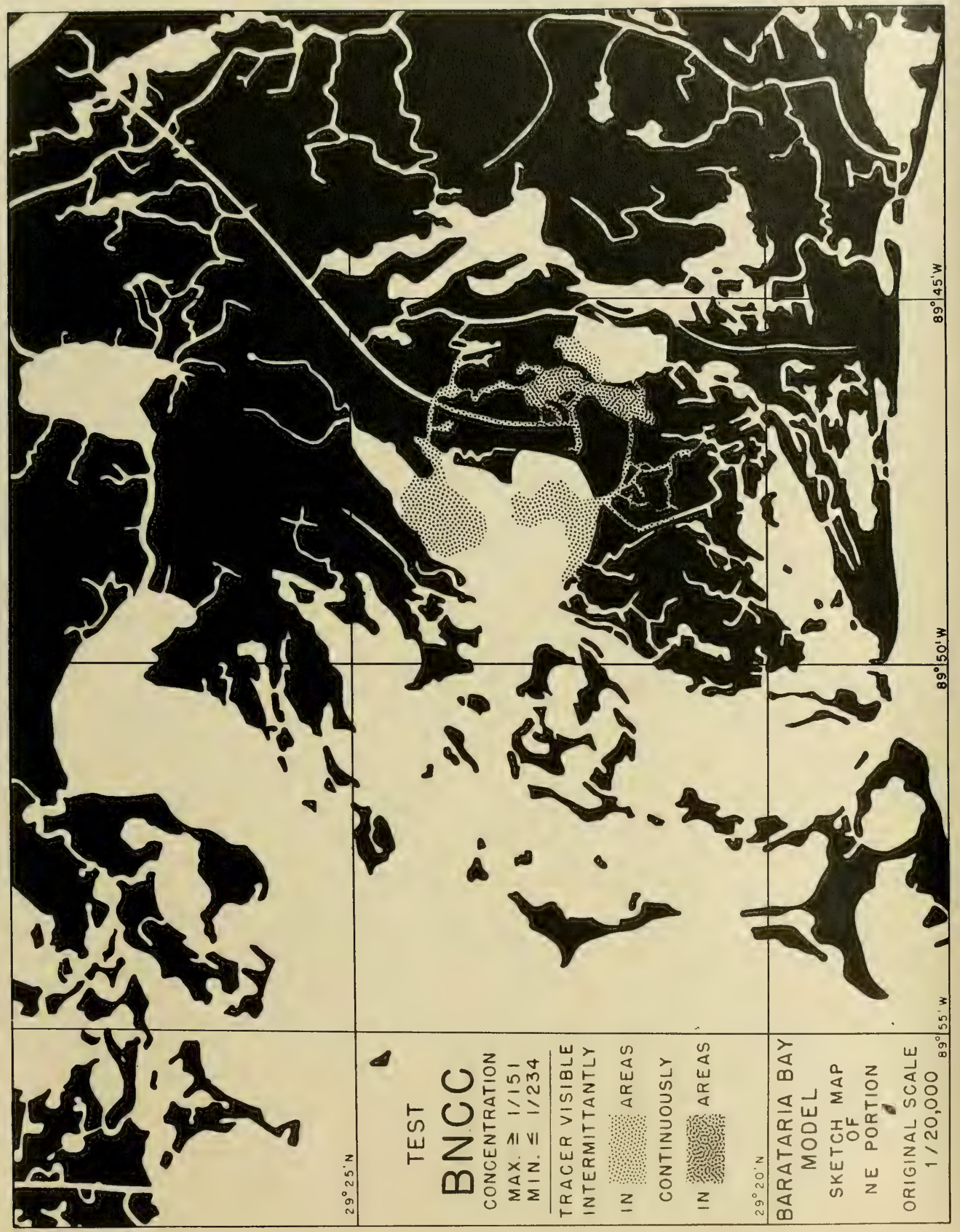




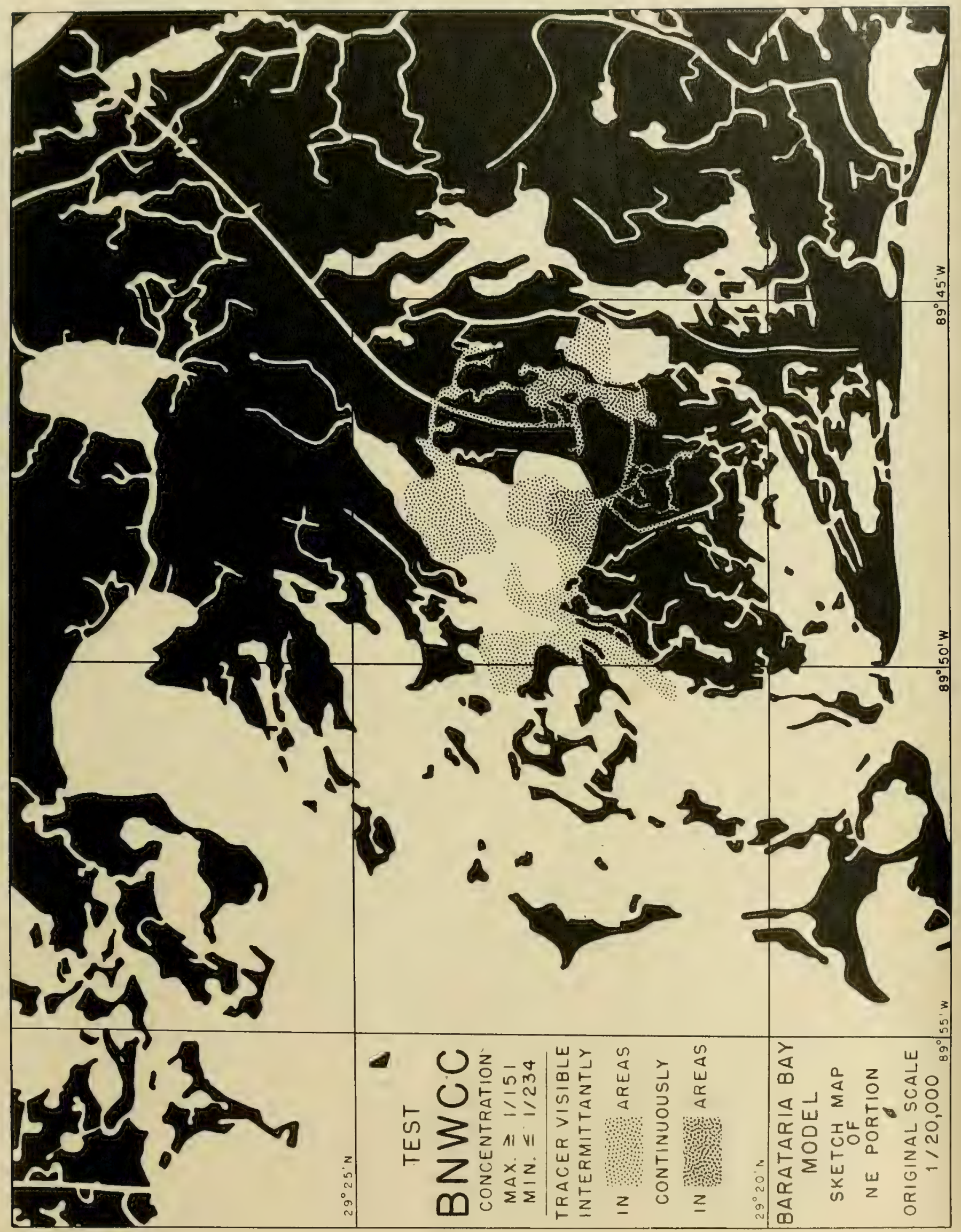





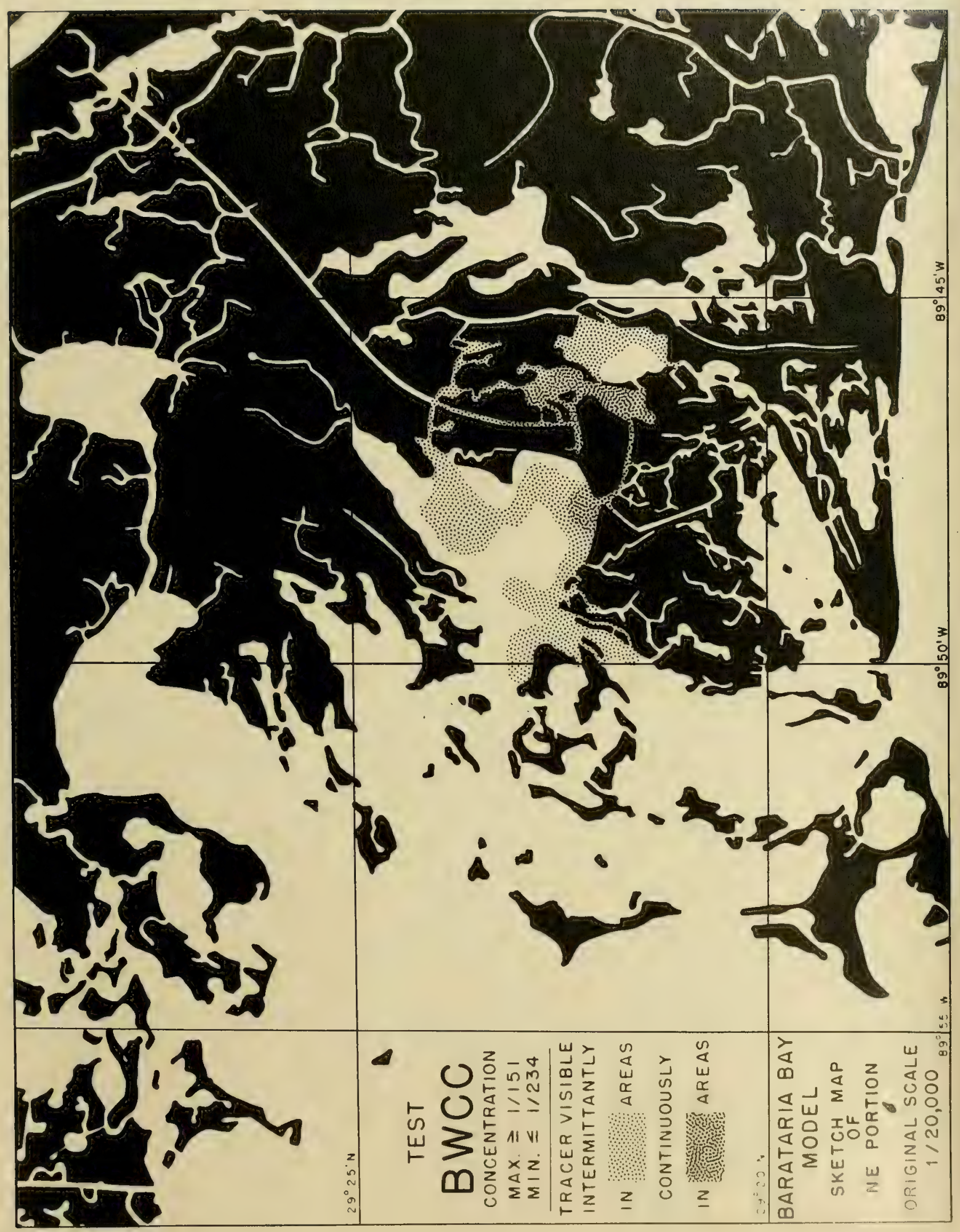





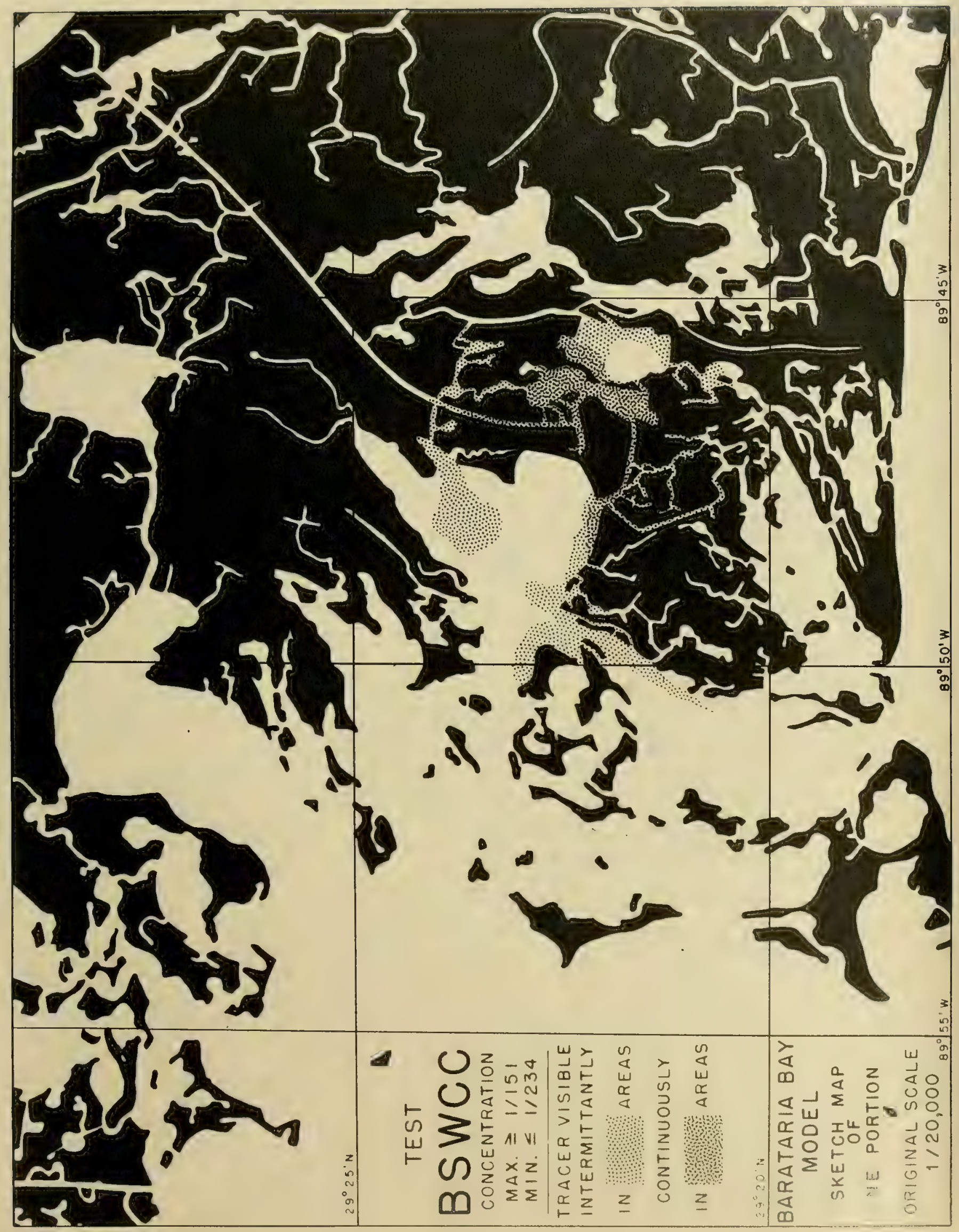





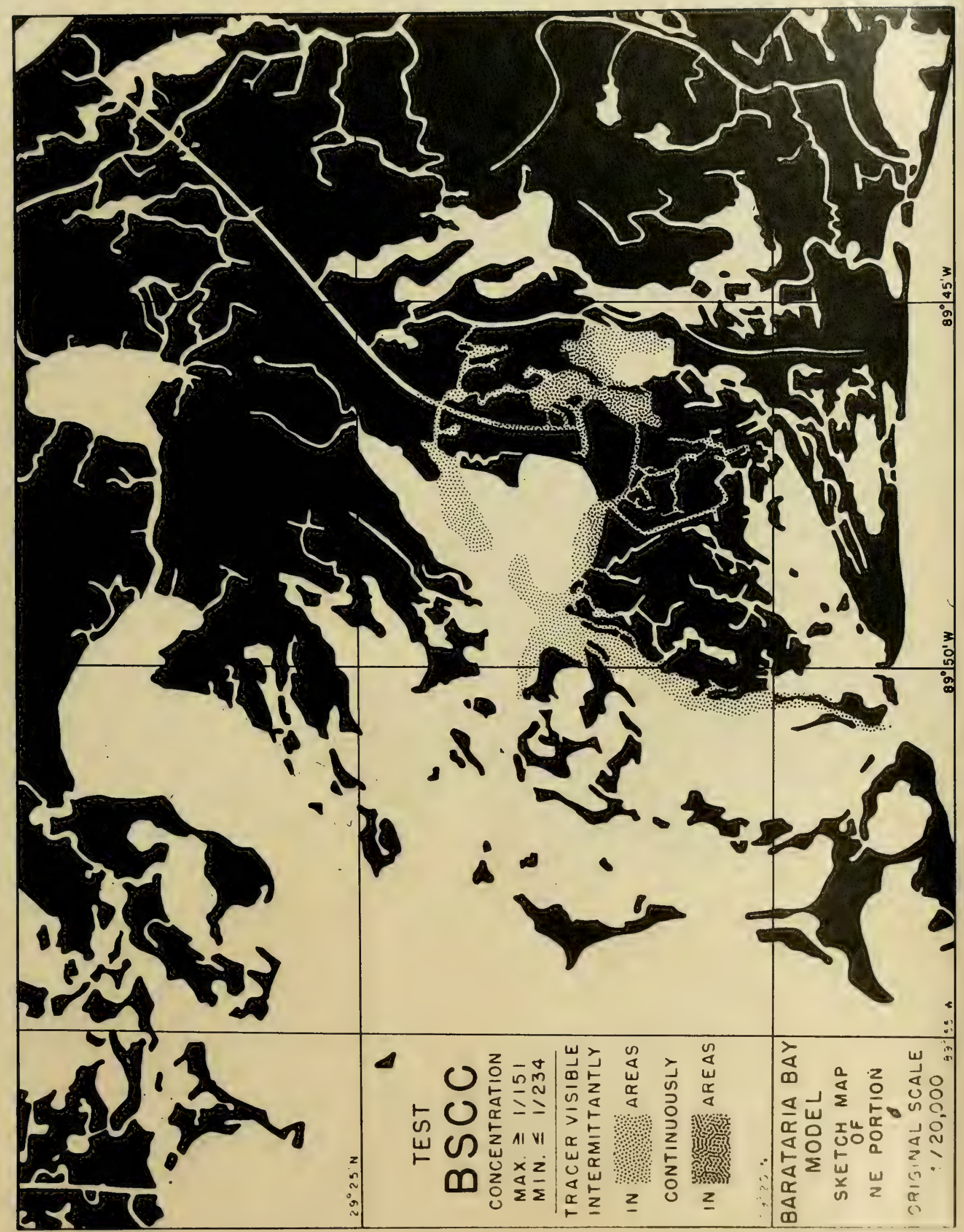





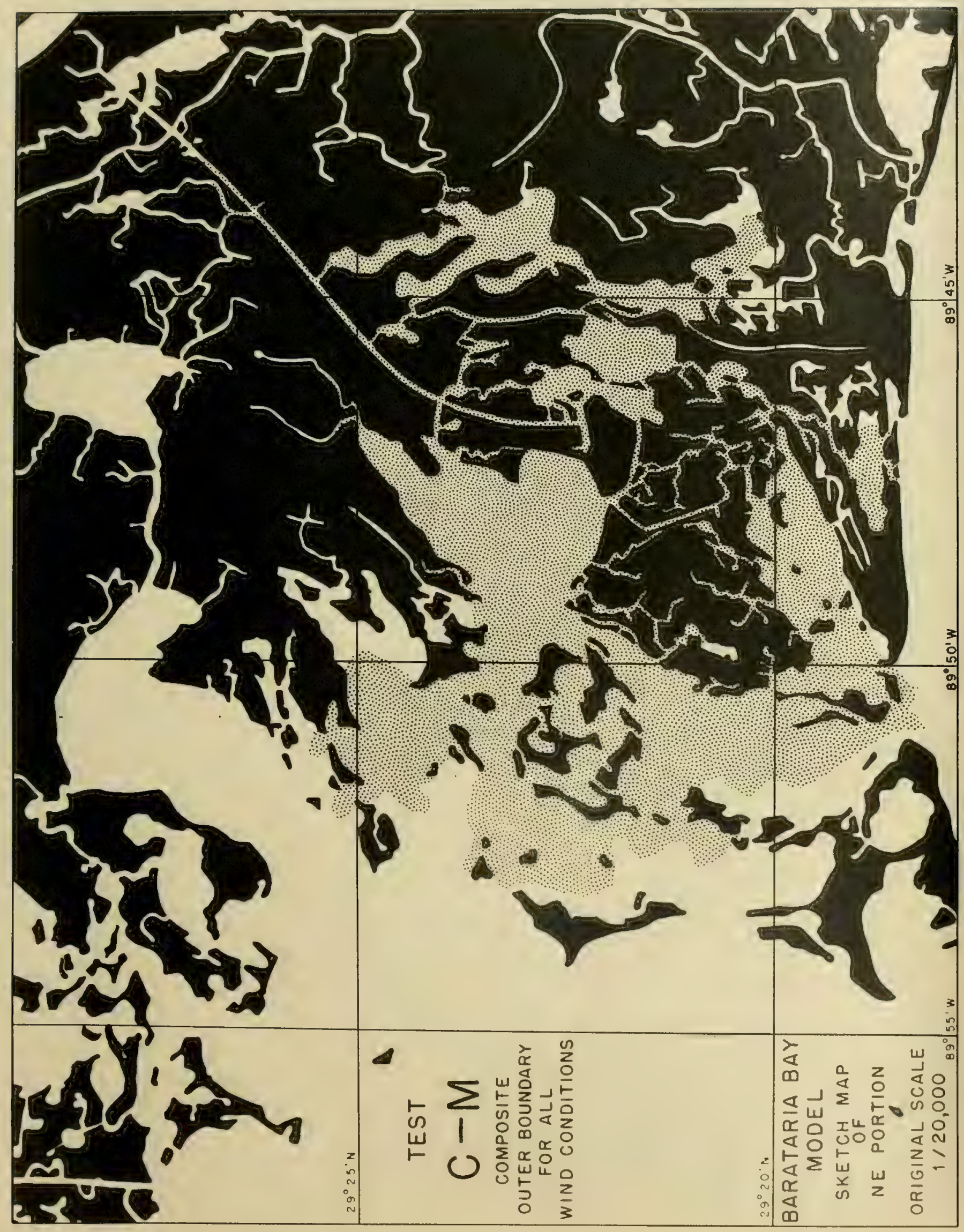





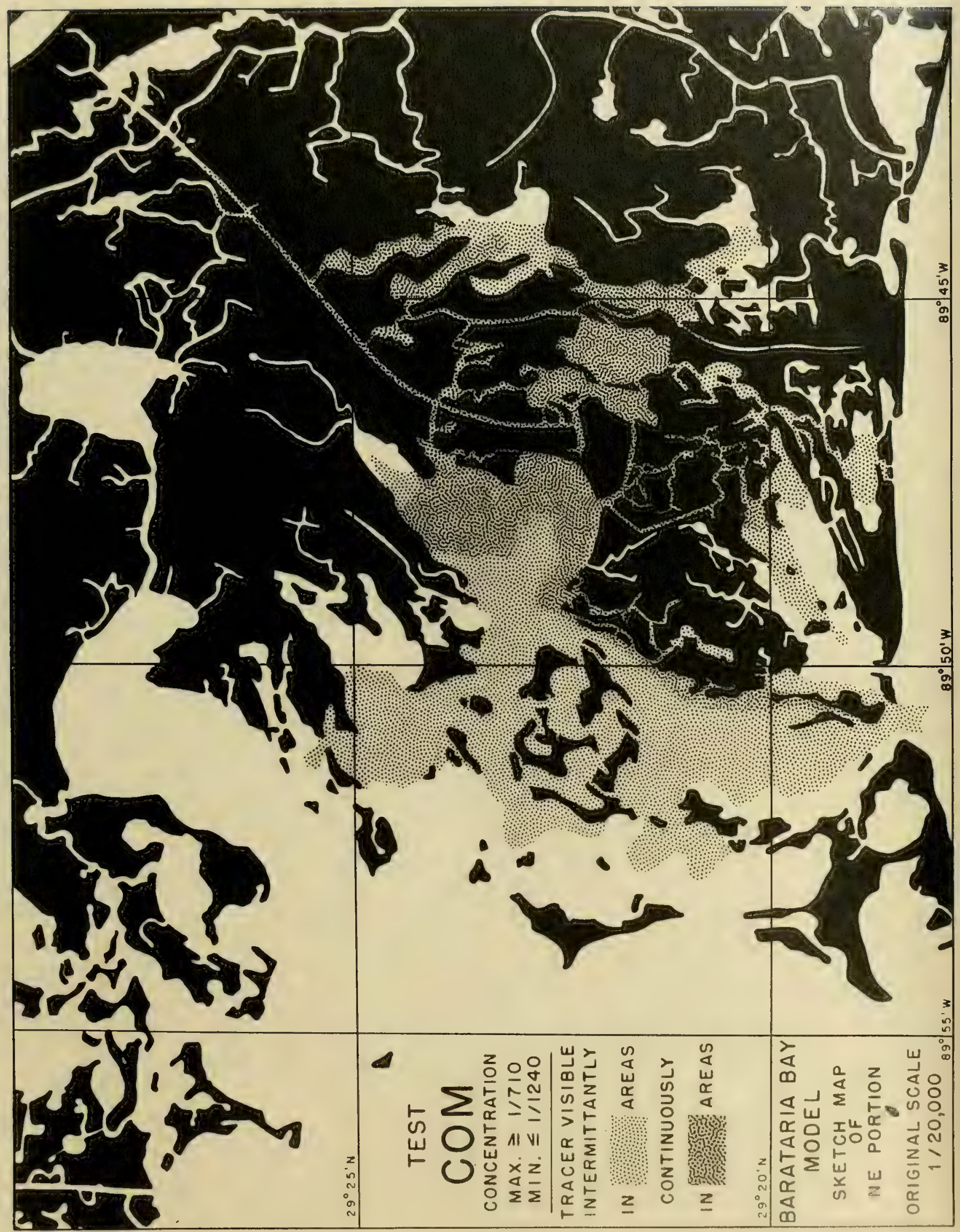





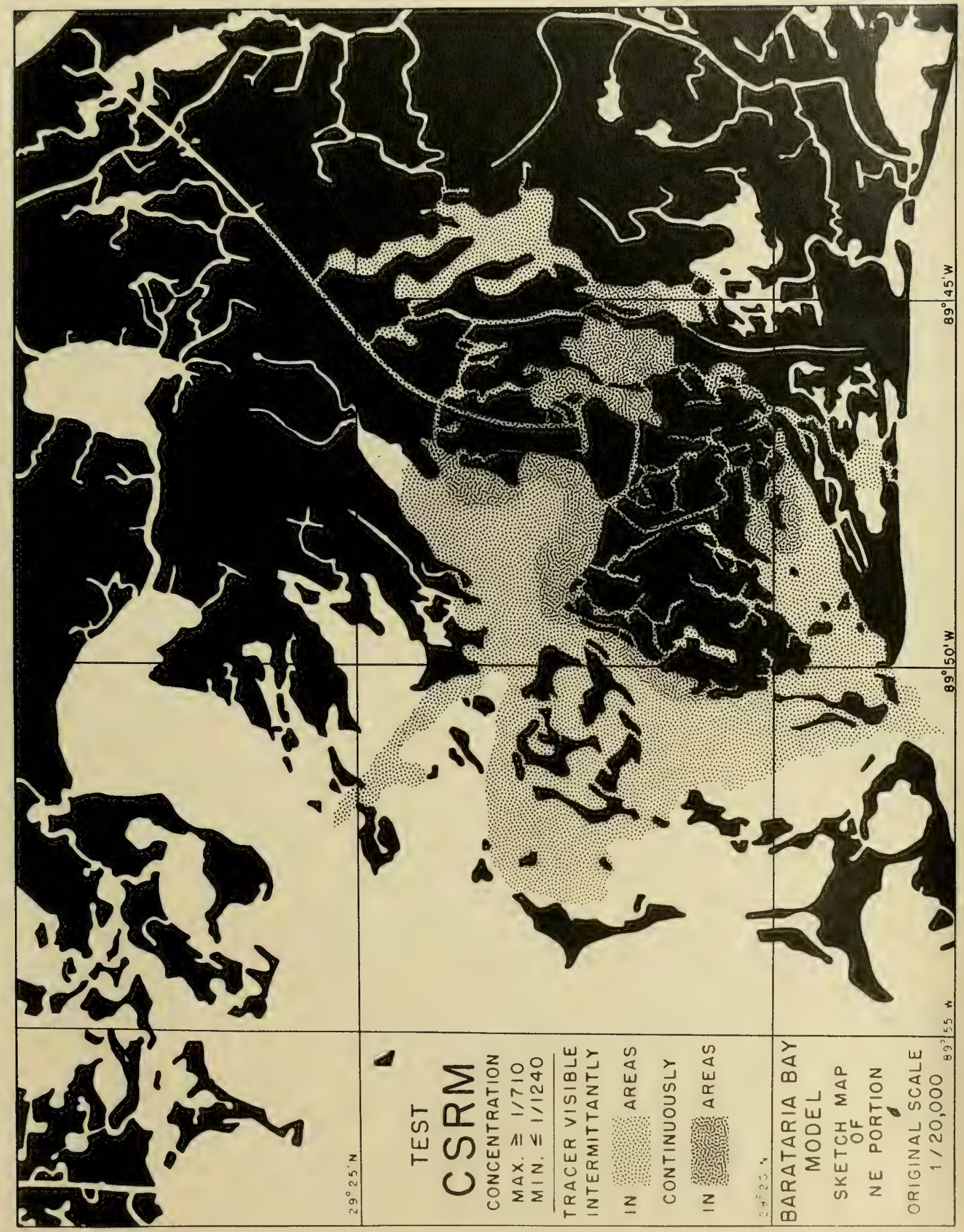





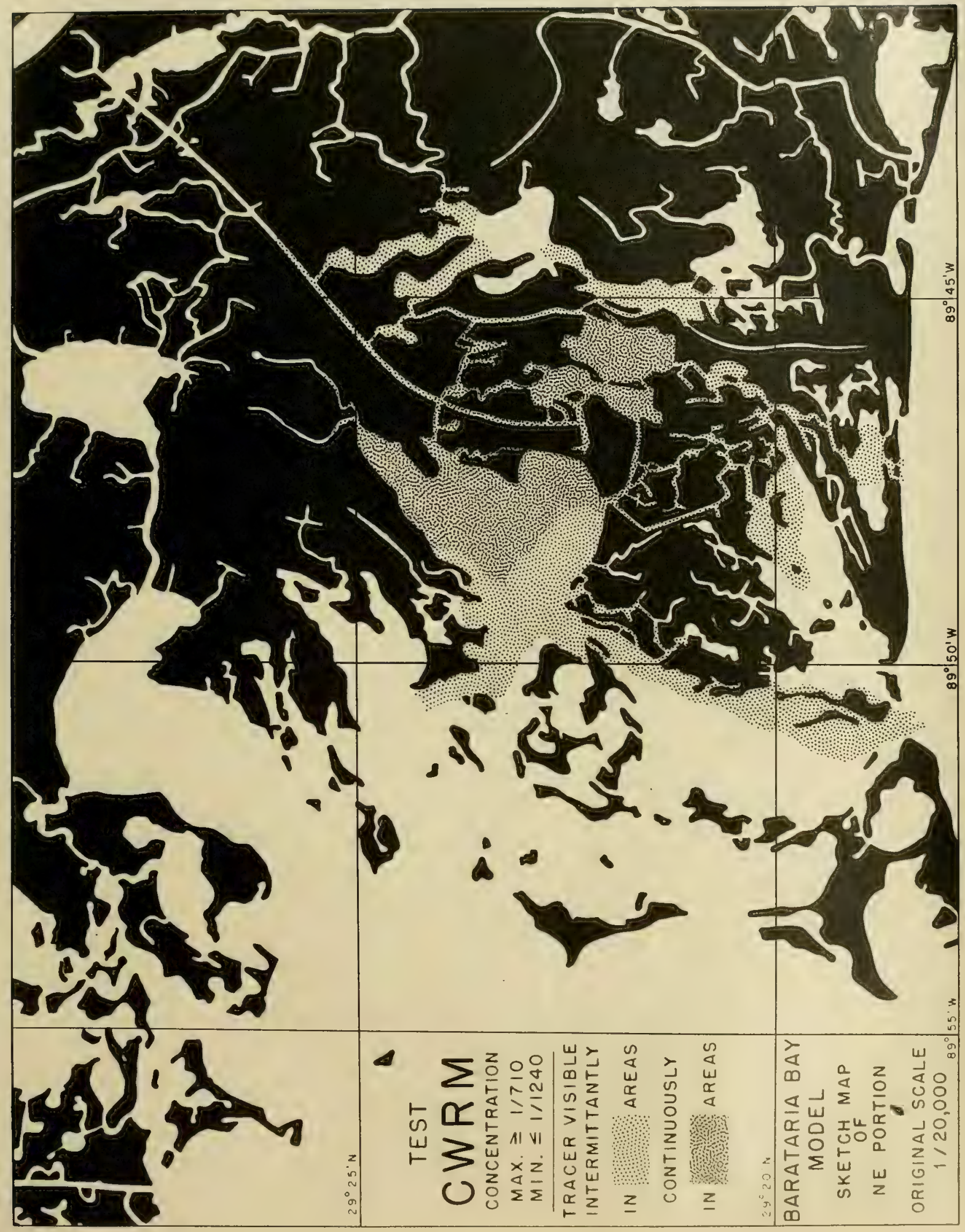





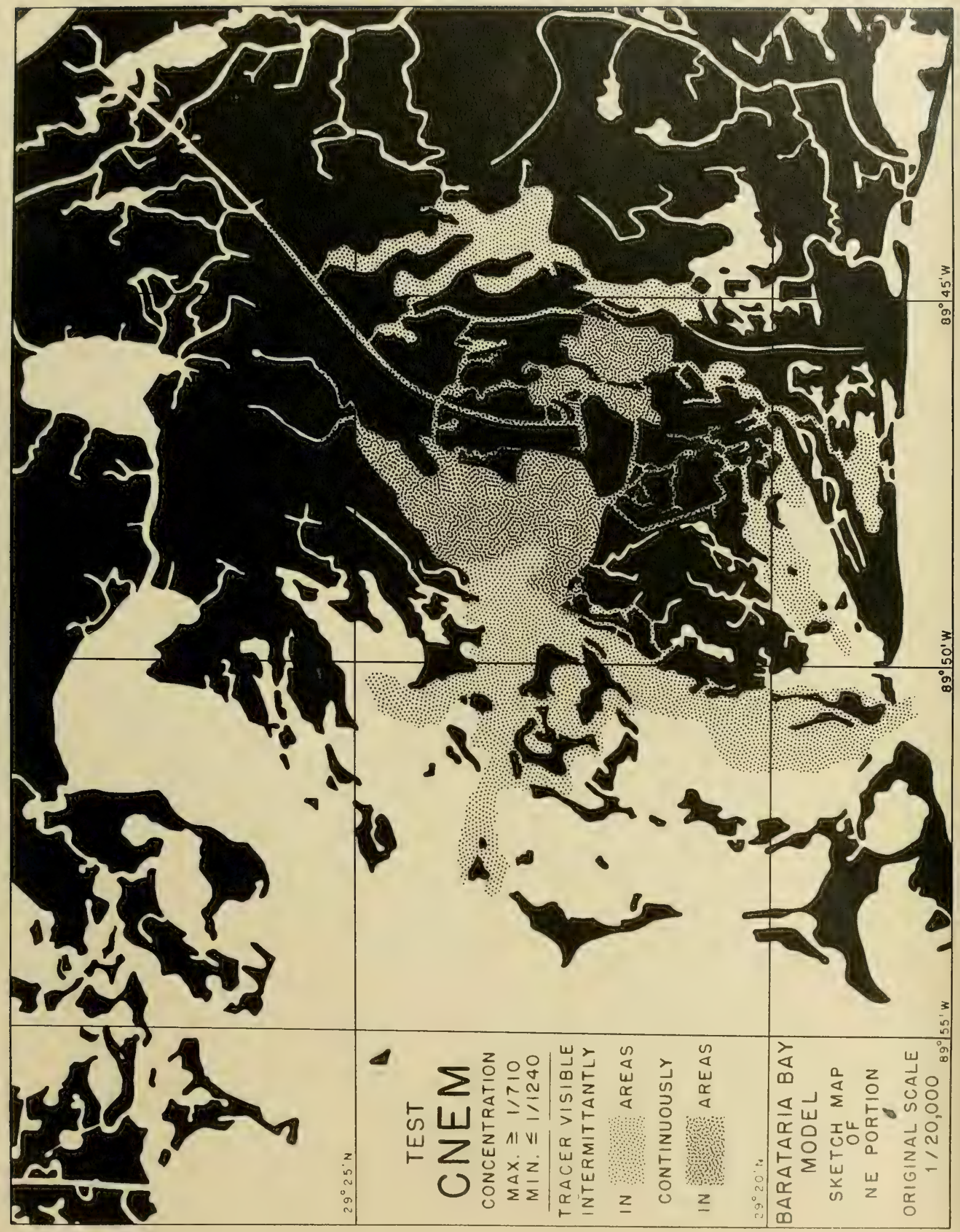





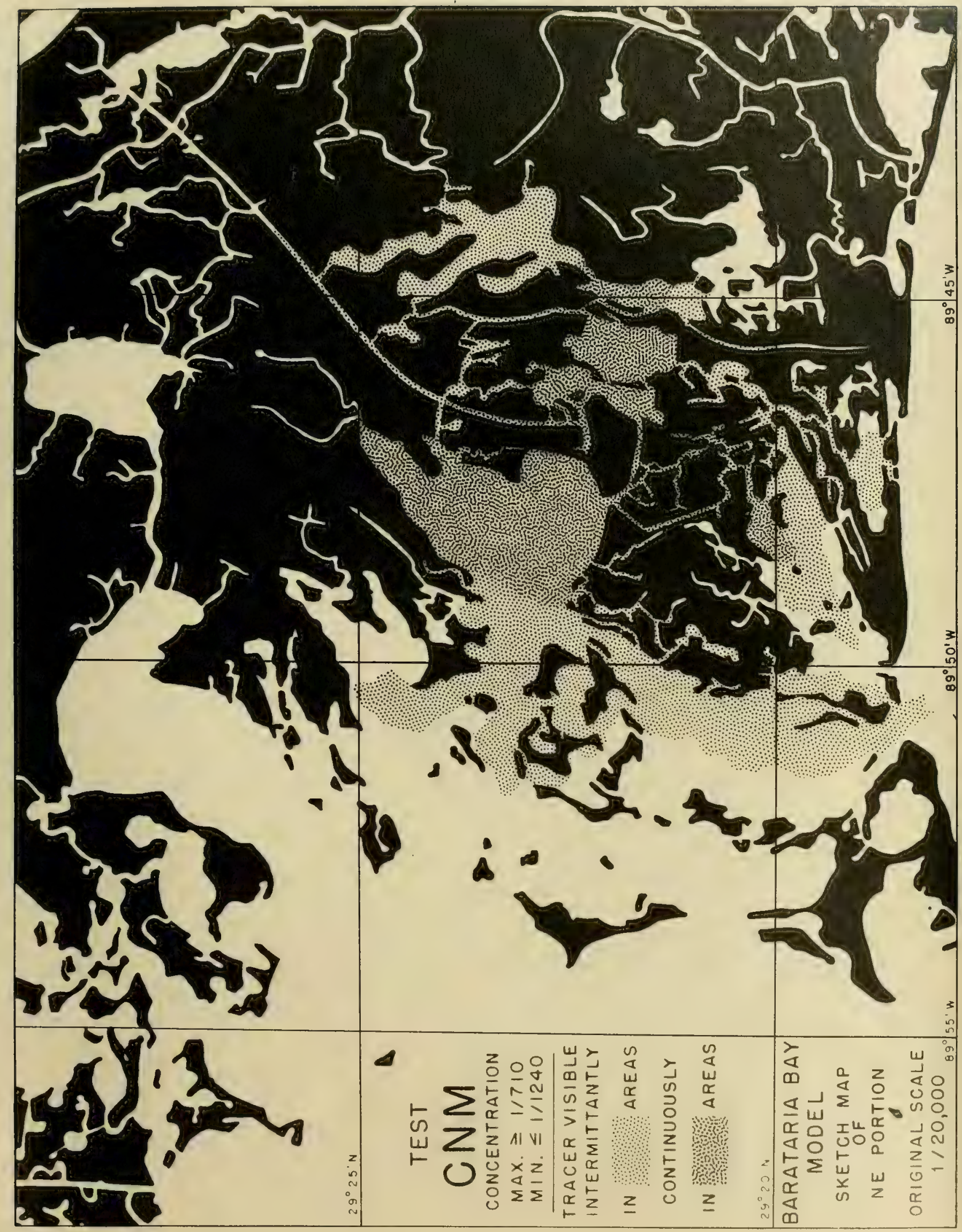





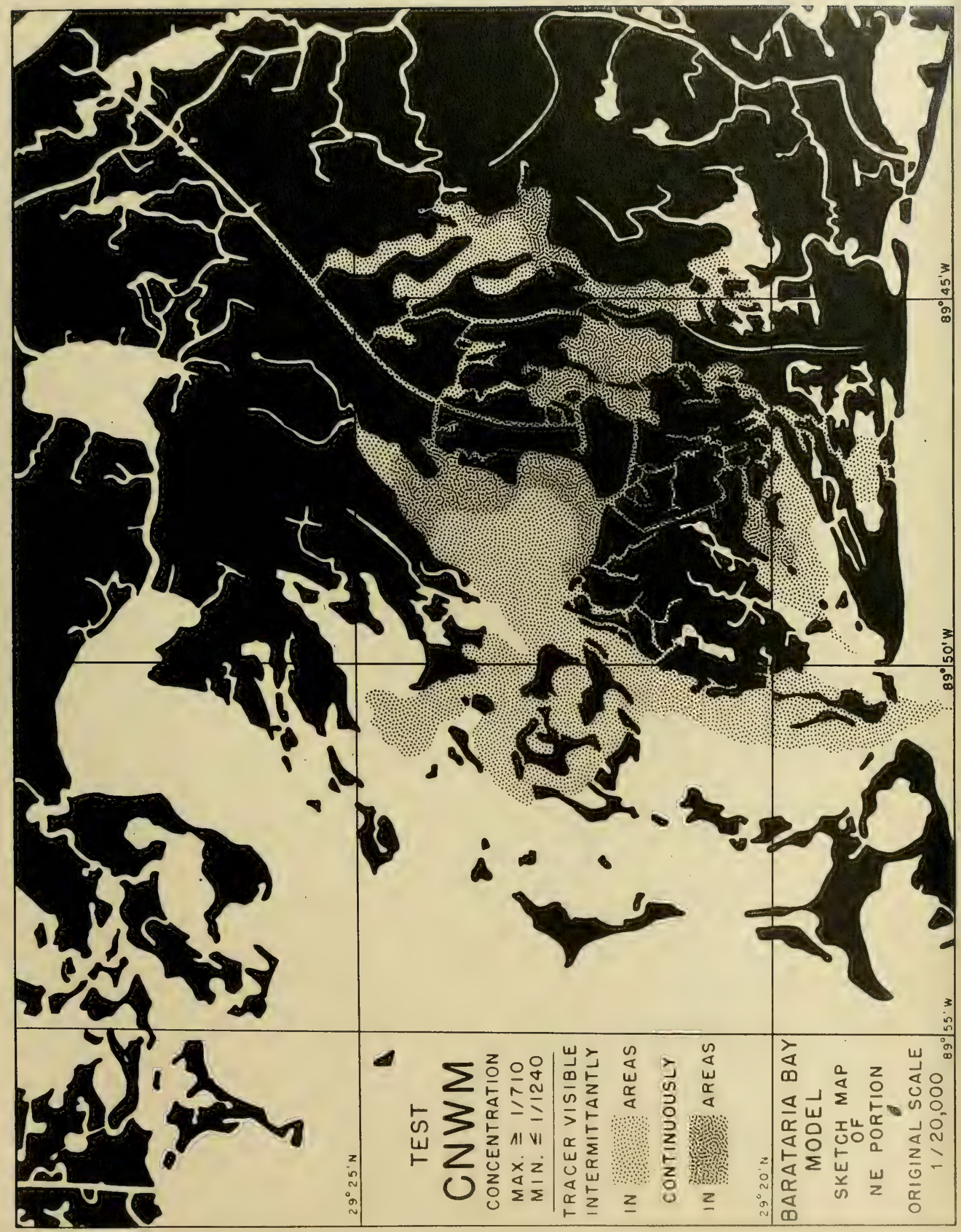





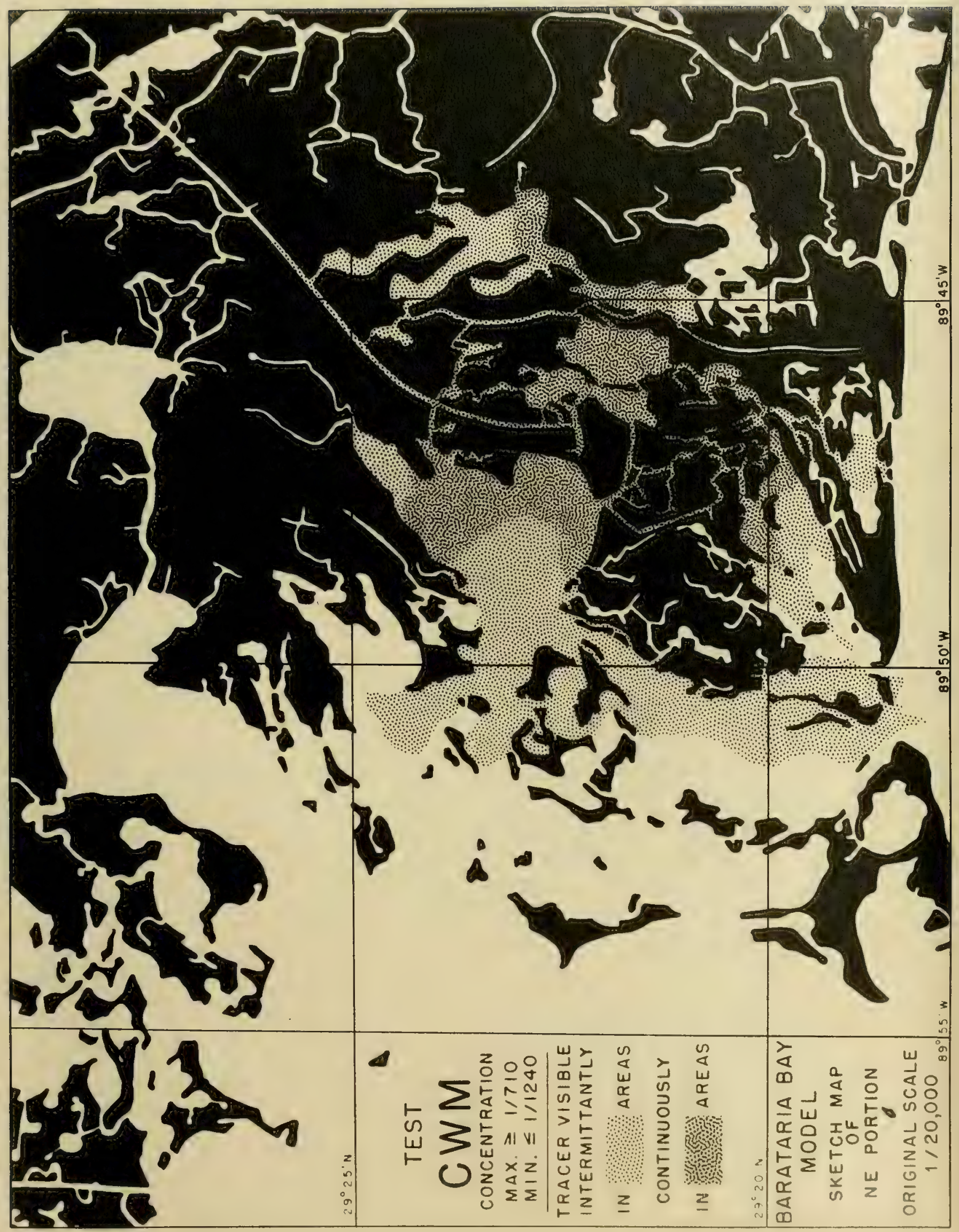





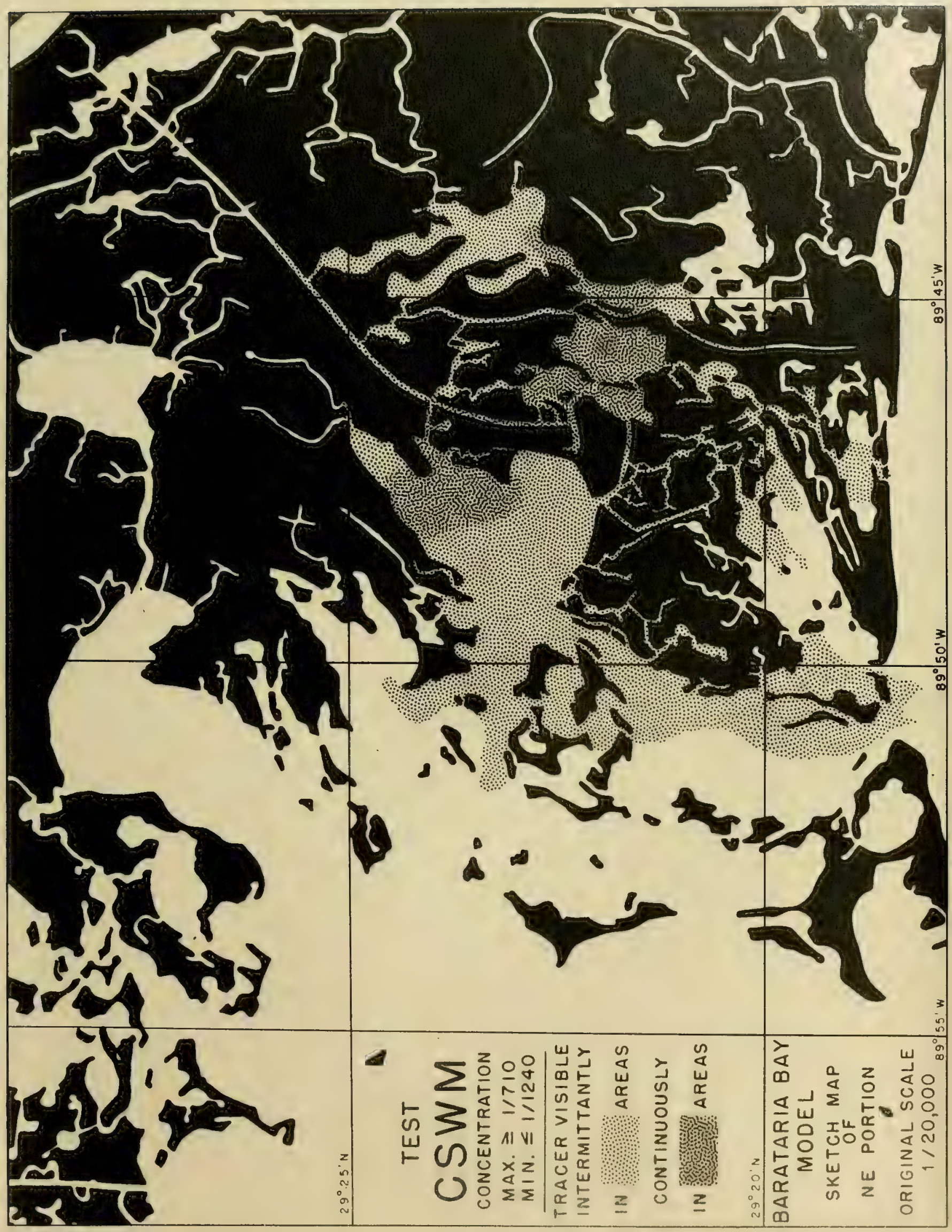





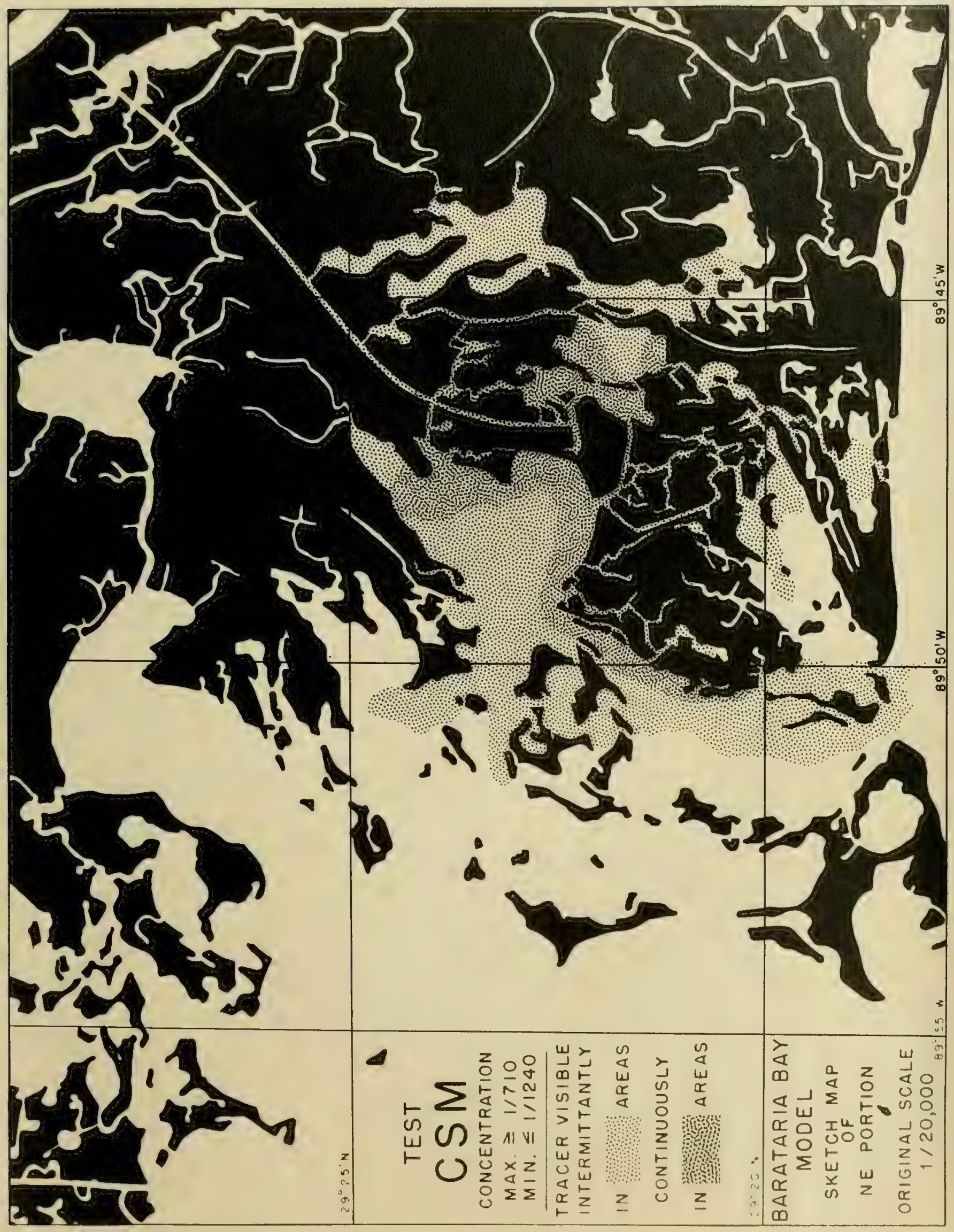





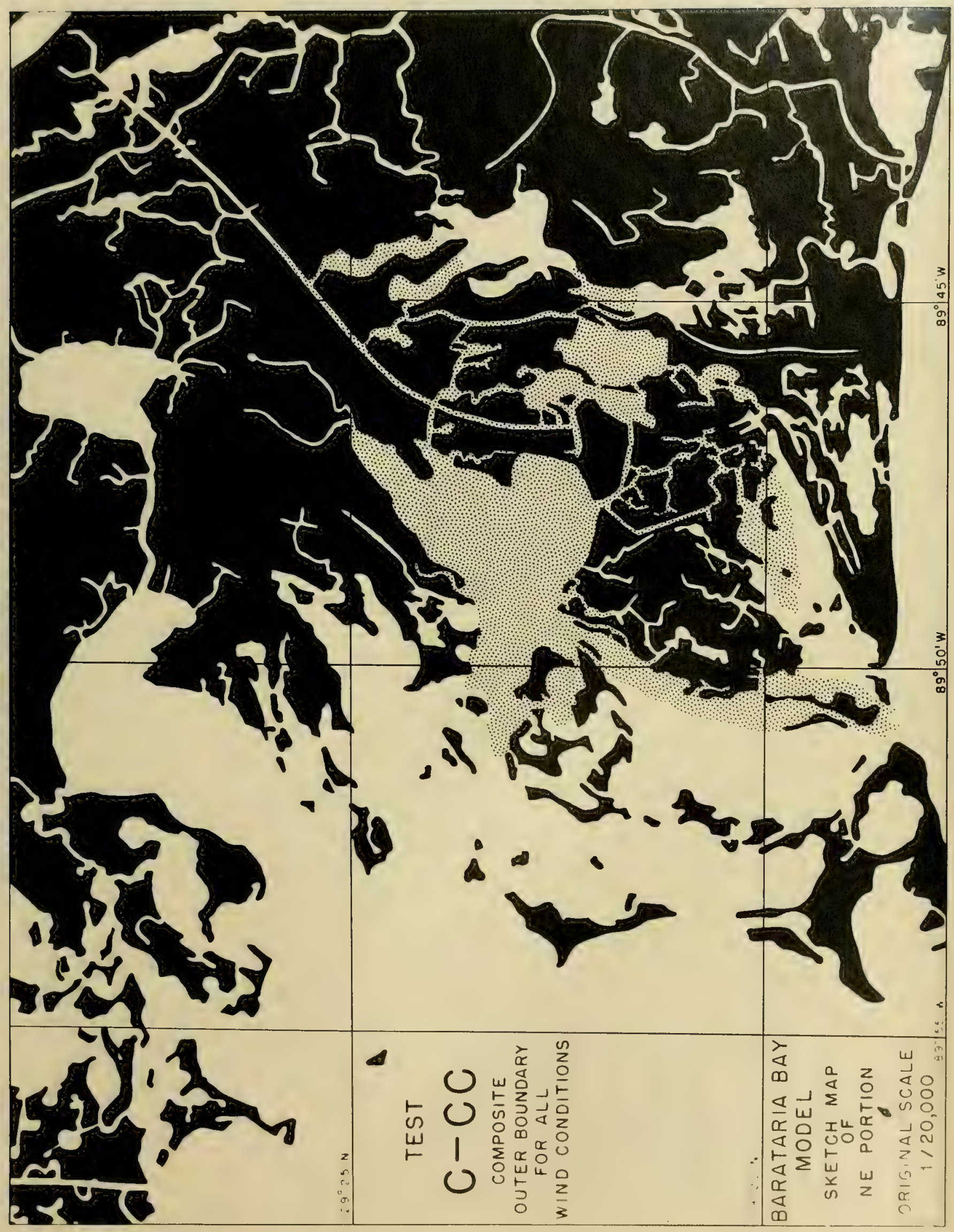





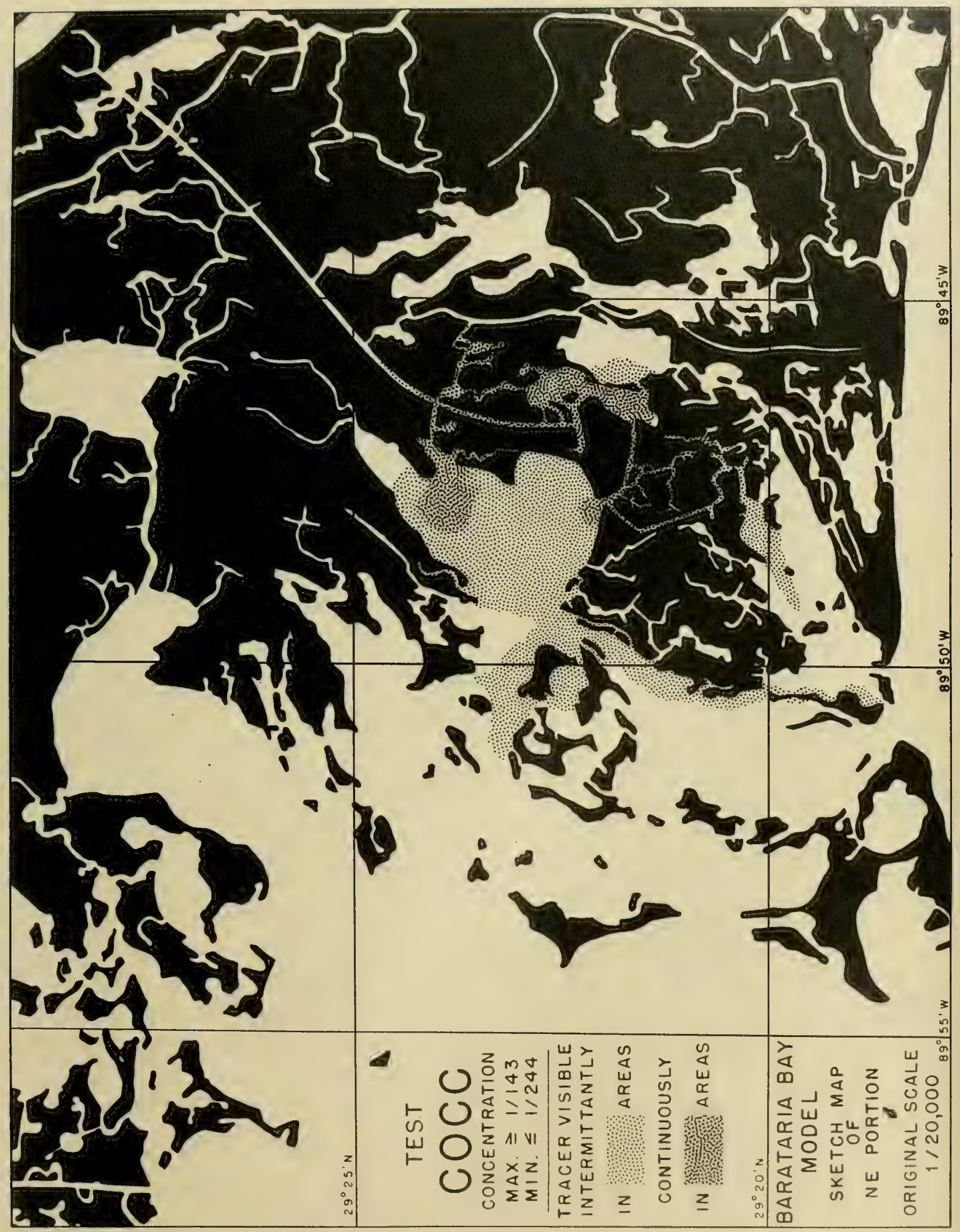





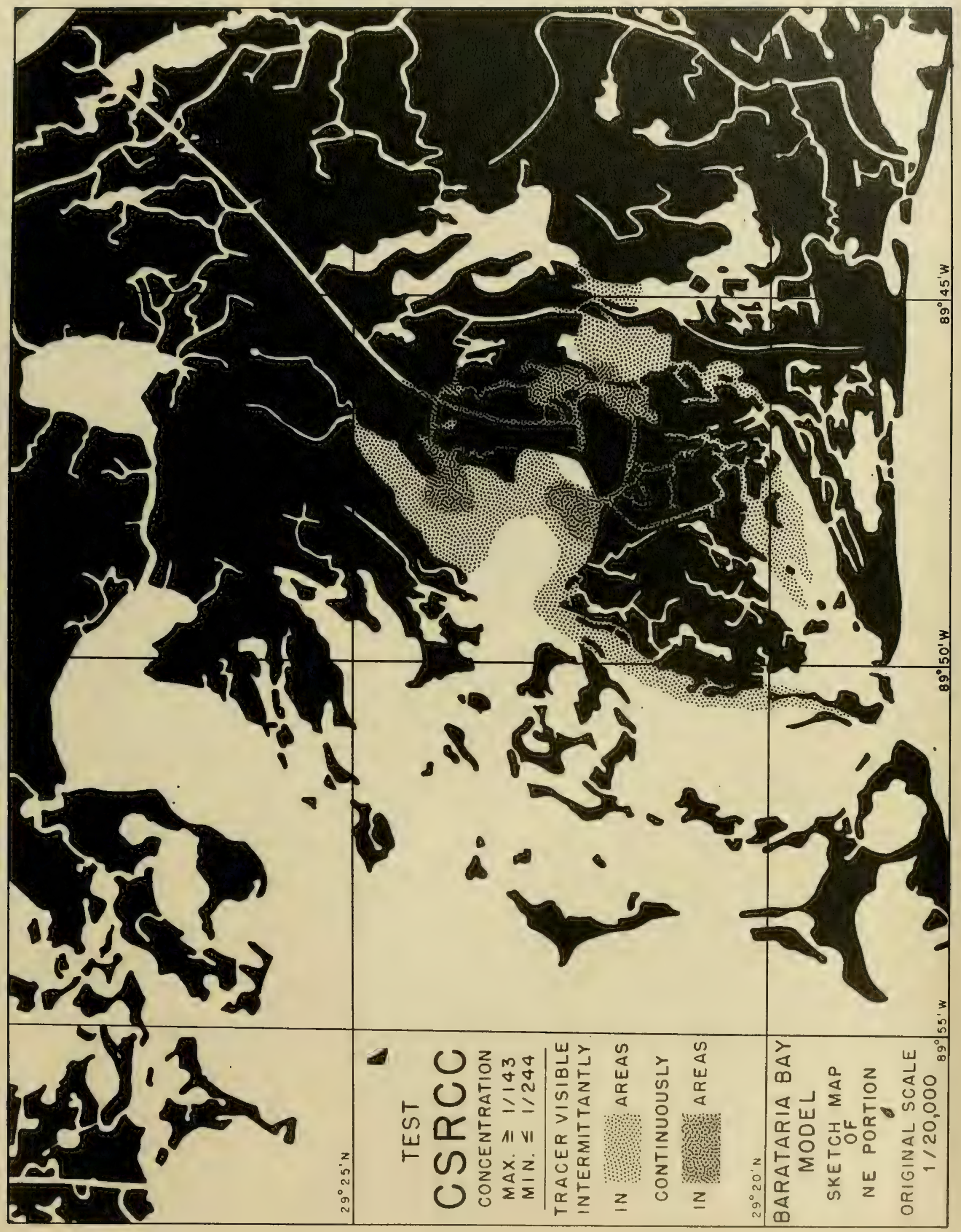





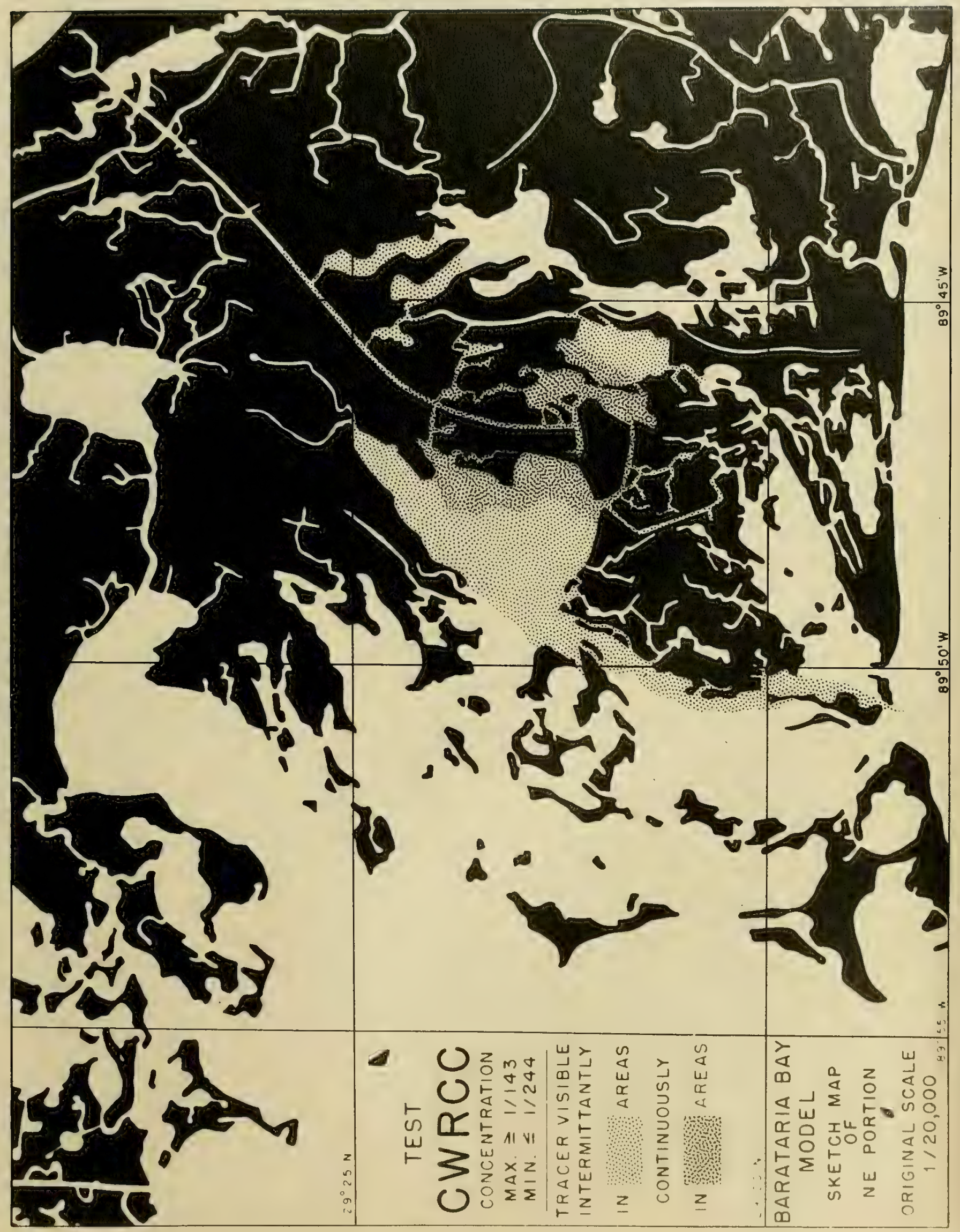





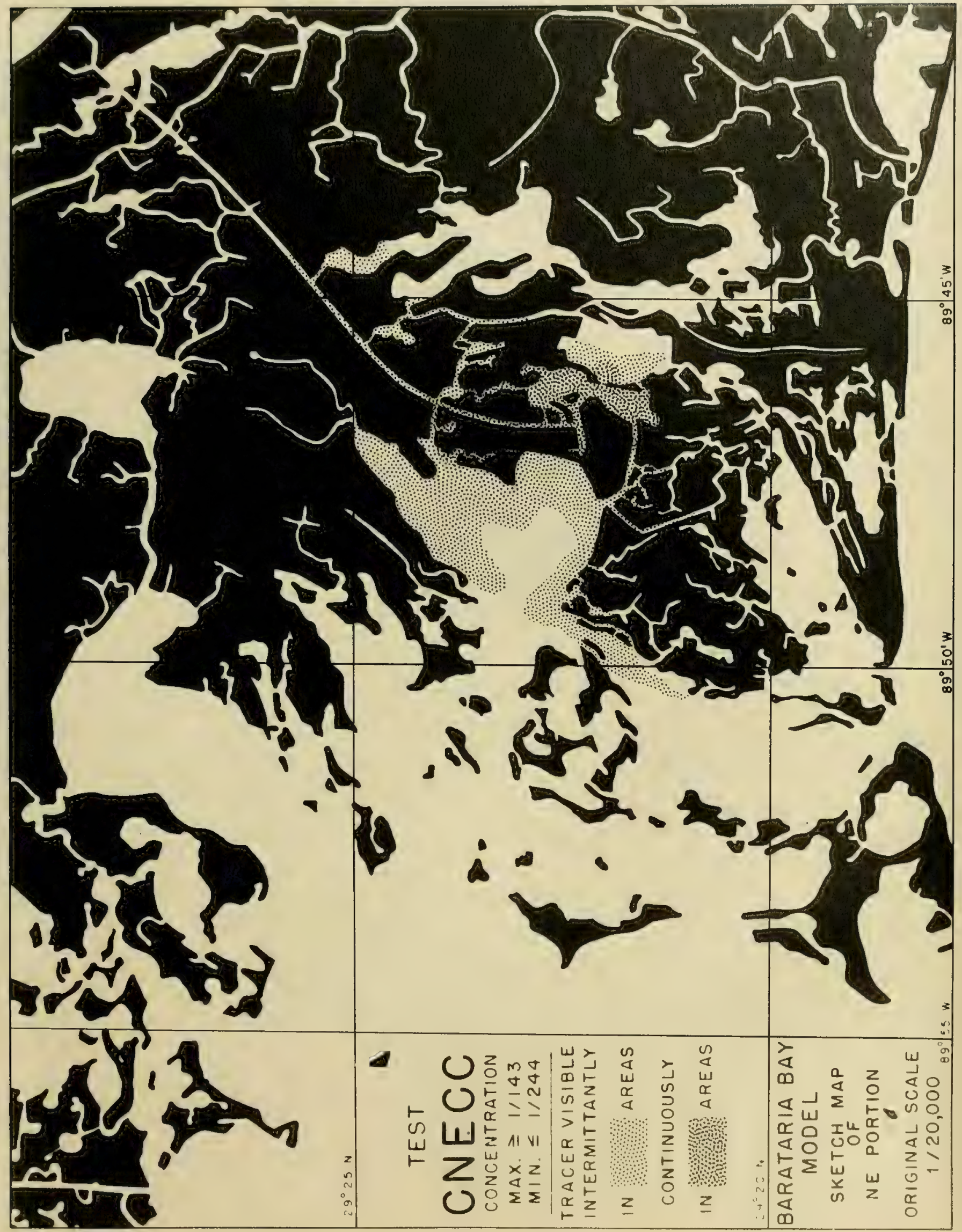





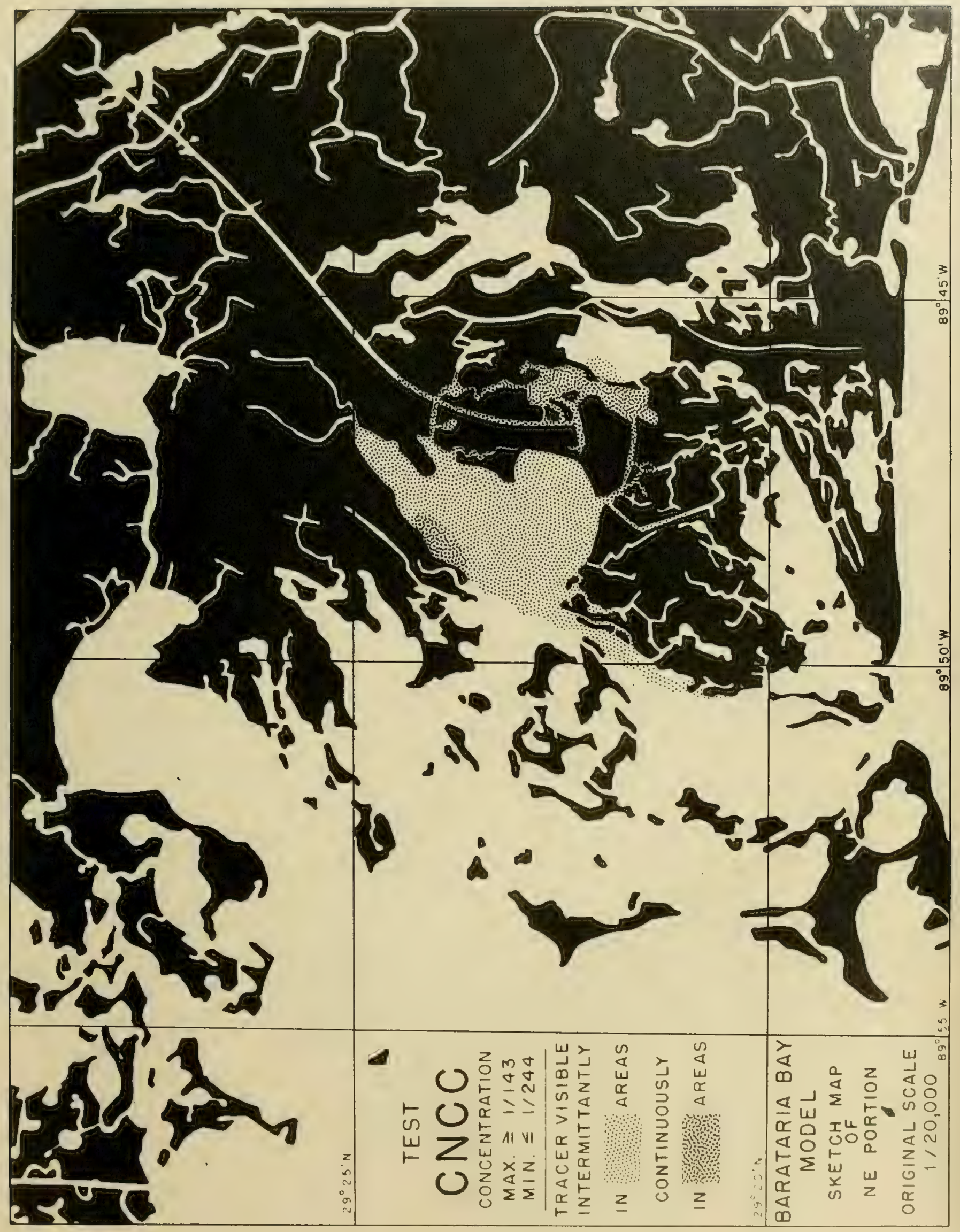





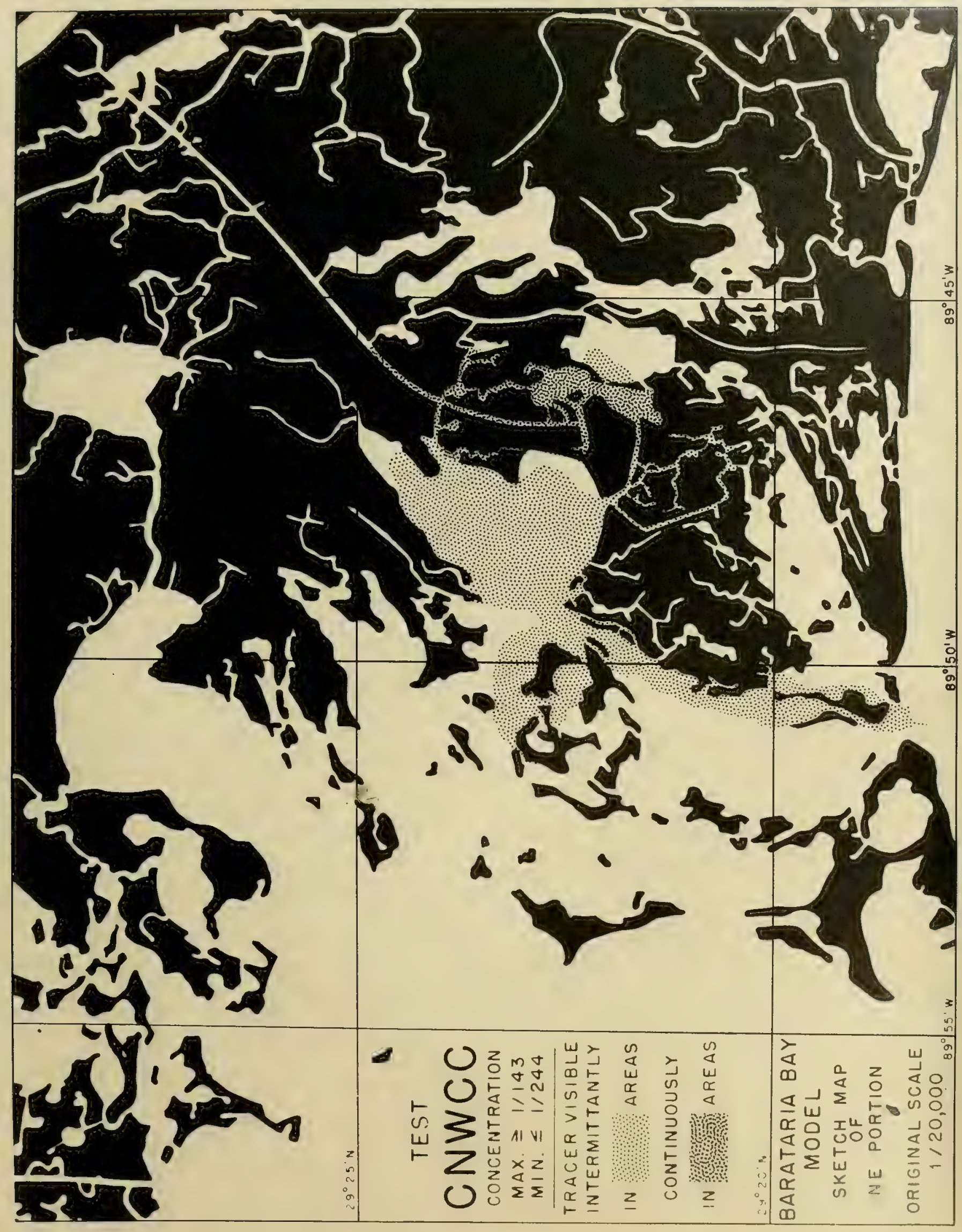





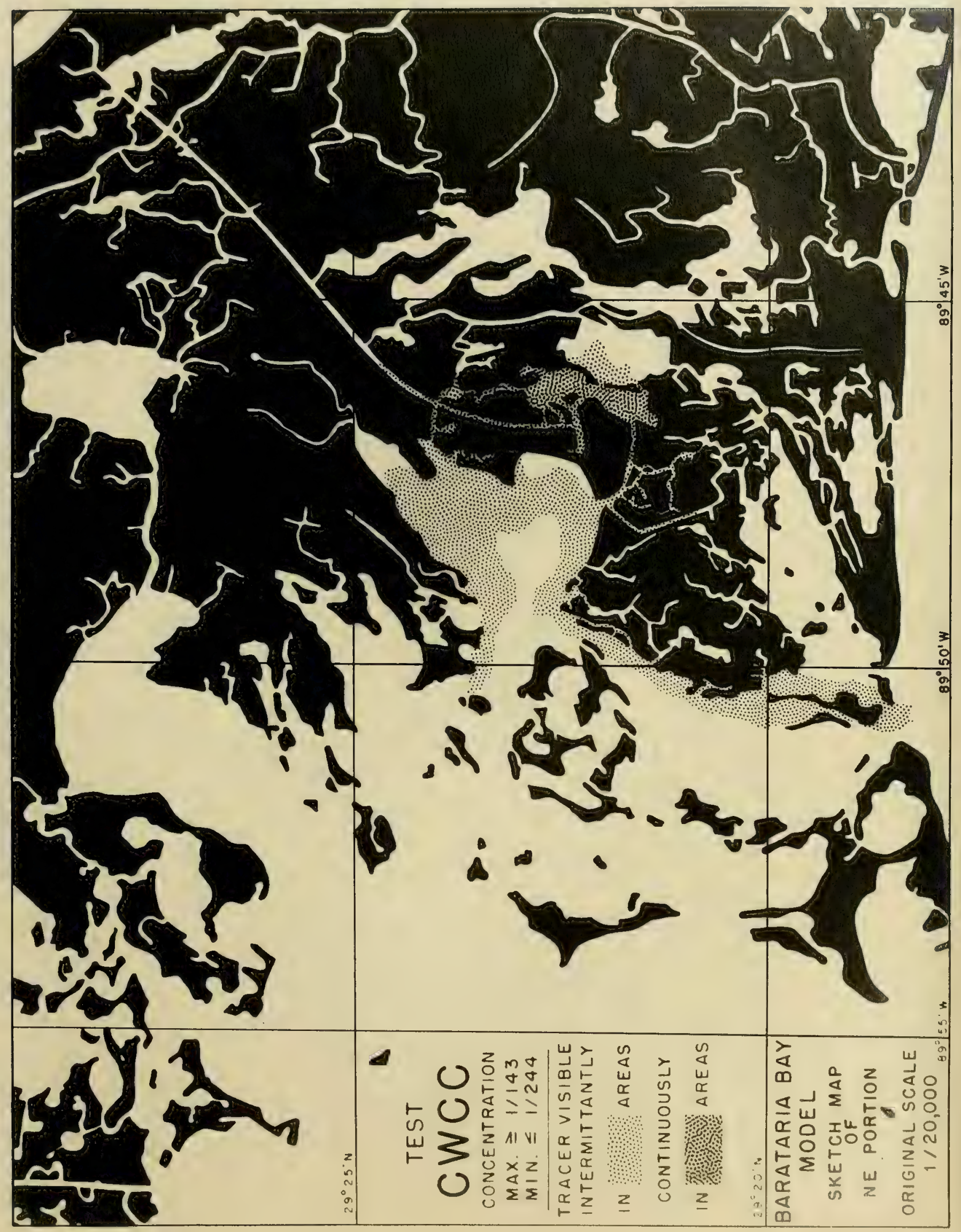





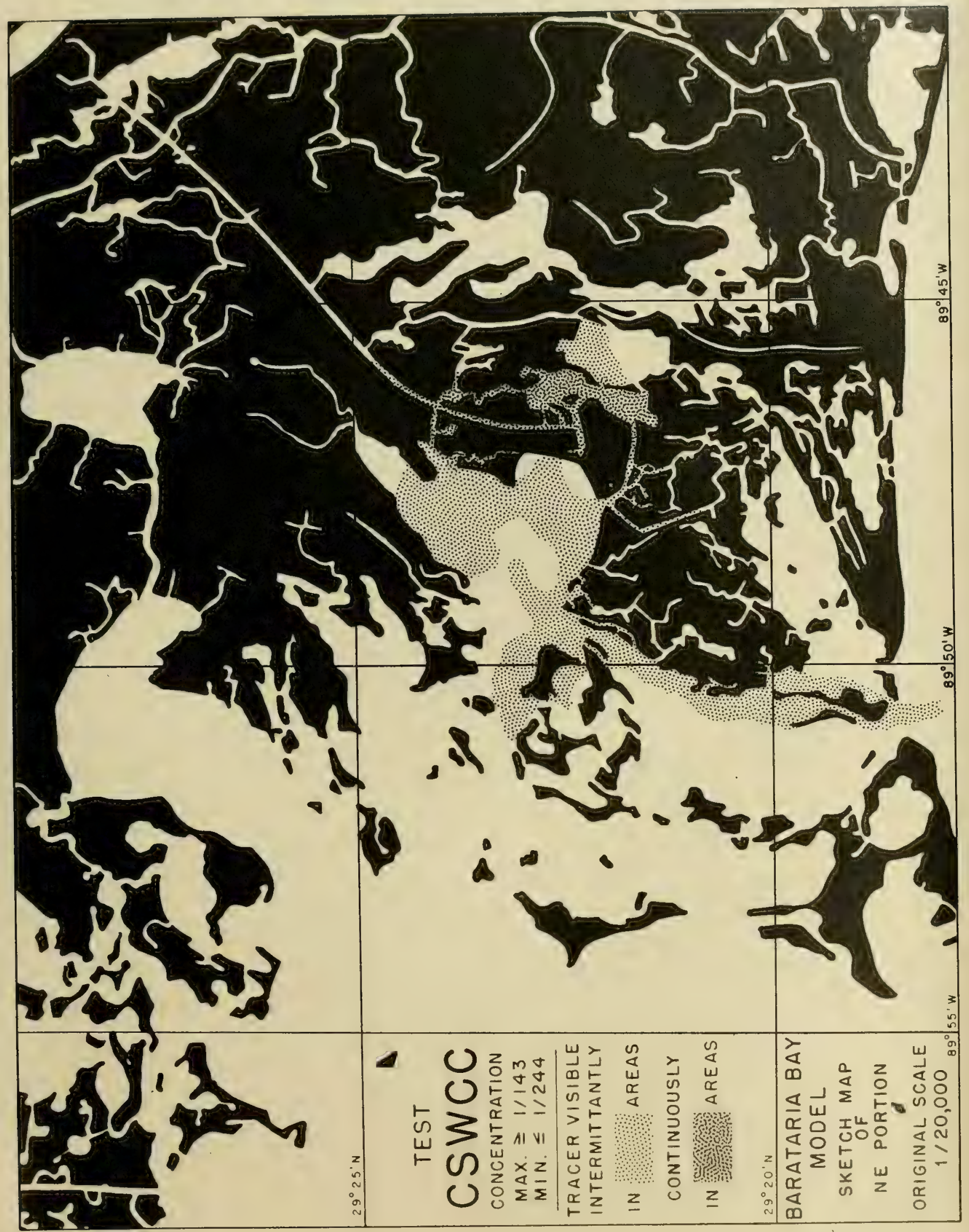





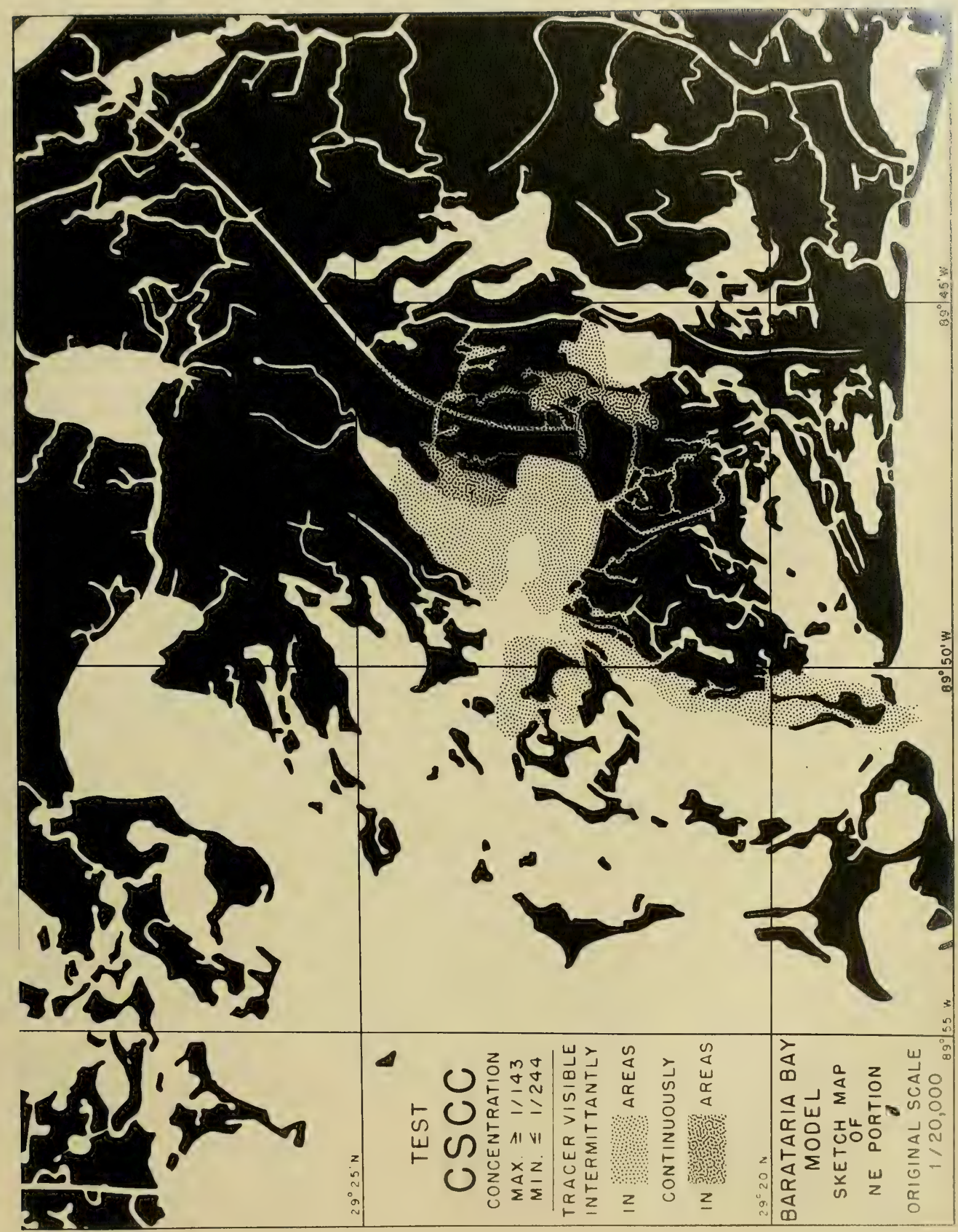





